\title{
Practicing the plastic brain
}

Citation for published version (APA):

van de Werff, T. (2018). Practicing the plastic brain: popular neuroscience and the good life. [Doctoral Thesis, Maastricht University]. Maastricht University. https://doi.org/10.26481/dis.20180516tw

Document status and date:

Published: 01/01/2018

DOI:

10.26481/dis.20180516tw

Document Version:

Publisher's PDF, also known as Version of record

\section{Please check the document version of this publication:}

- A submitted manuscript is the version of the article upon submission and before peer-review. There can be important differences between the submitted version and the official published version of record.

People interested in the research are advised to contact the author for the final version of the publication, or visit the DOI to the publisher's website.

- The final author version and the galley proof are versions of the publication after peer review.

- The final published version features the final layout of the paper including the volume, issue and page numbers.

Link to publication

\footnotetext{
General rights rights.

- You may freely distribute the URL identifying the publication in the public portal. please follow below link for the End User Agreement:

www.umlib.nl/taverne-license

Take down policy

If you believe that this document breaches copyright please contact us at:

repository@maastrichtuniversity.nl

providing details and we will investigate your claim.
}

Copyright and moral rights for the publications made accessible in the public portal are retained by the authors and/or other copyright owners and it is a condition of accessing publications that users recognise and abide by the legal requirements associated with these

- Users may download and print one copy of any publication from the public portal for the purpose of private study or research.

- You may not further distribute the material or use it for any profit-making activity or commercial gain

If the publication is distributed under the terms of Article $25 \mathrm{fa}$ of the Dutch Copyright Act, indicated by the "Taverne" license above, 
Ties van

de Werff

\section{PRACTICING THE PLASTIC BRAIN}

\section{Popular neuroscience \\ and the good life}

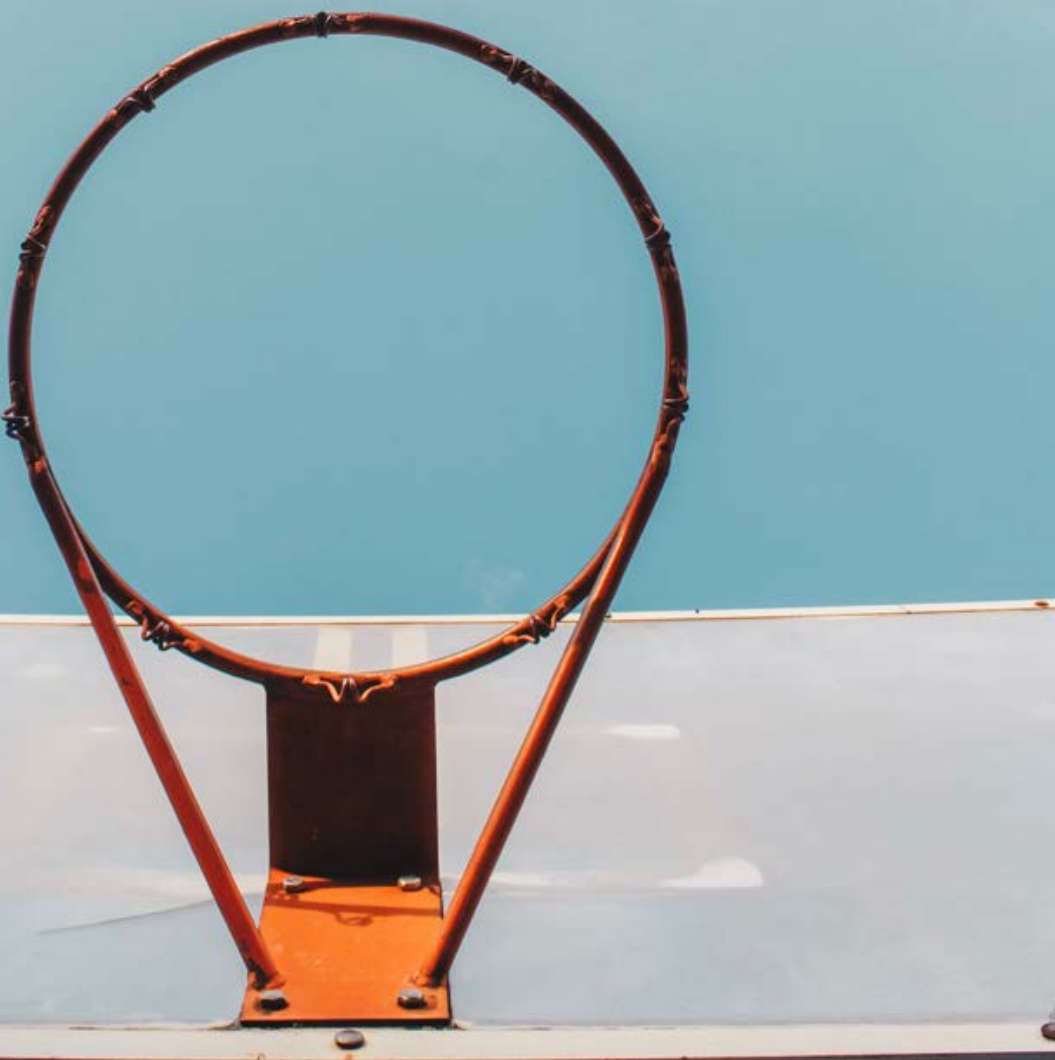


ISBN 978-94-6295-885-2

NUR 730

Cover design: Naomi Naus

Printing and layout: Datawyse Maastricht

(C) Ties van de Werff, 2018

The printing of this dissertation has been financially supported by the Netherlands Graduate Research School of Science, Technology and Modern Culture (WTMC). 


\title{
PRACTICING THE PLASTIC BRAIN popular neuroscience and the good life
}

\author{
Proefschrift
}

ter verkrijging van de graad van doctor aan de Universiteit Maastricht, op gezag van de Rector Magnificus, Prof.dr. Rianne M. Letschert volgens het besluit van het College van Decanen,

in het openbaar te verdedigen

op woensdag 16 mei 2018 om 10.00 uur

door

Ties van de Werff 


\section{Promotores:}

Prof. dr. T. E. Swierstra

Prof. dr. H. J. Pott (Erasmus Universiteit Rotterdam)

\section{Beoordelingscommissie:}

Prof. dr. ir. H. van Lente (voorzitter)

Prof. dr. K.T. Bijsterveld

Dr. M. Boenink (Universiteit Twente)

Prof. dr. J. de Mul (Erasmus Universiteit Rotterdam) 


\section{CONTENTS}

Acknowledgements

Chapter 1 Brain Plasticity and the Question of Moral Change 9

1. Introduction 9

2. Promises and Perils of a Plastic Brain 11

3. The Plastic Brain as Medicine for Living Well 19

4. Three Cases of a Brain-inspired Good Life 28

Chapter 2 Acting as a Good External Frontal Lobe: parenting teenage brains 35

1. Introduction 35

2. A Brief History of Parenting and Adolescence 38

3. Adolescent Brain Development and the Maturing Prefrontal Cortex Thesis

4. The Teenage Brain as Parenting Advice 47

5. Taming the Future: moral changes in parenting practices 56

6. Conclusion 64

Chapter 3 A Monk at the Office: stress and the mindful brain in the $\begin{array}{ll}\text { workplace } & 67\end{array}$

1. Introduction $\quad 67$

2. A History of Work Ethic and Science-based Management 70

3. The Mindful Brain: from Buddhist practice to brain training 75

4. The Mindful Brain at the Office 84

5. Wholesome Working in a Technologized Work Culture 93

$\begin{array}{ll}\text { 6. Conclusion } & 101\end{array}$ 
Chapter 4 Engaging the Ageing Brain: cognitive decline and ageing well 105 1. Introduction 105

2. Ageing Well Throughout History 108

3. Brain Games for Seniors: rejuvenating the ageing brain 113

4. From Fragile to Agile: repertoires of an ageing brain 119

5. Successful Brain Ageing in a Hypercognitive Society 131

6. Conclusion 137

Chapter 5 Practicing the Plastic Brain 141

1. Introduction 141

2. Changing Brains, Changing Moralities? 143

3. Conclusion 151

$\begin{array}{ll}\text { List of Figures } & 157\end{array}$

$\begin{array}{ll}\text { References } & 159\end{array}$

$\begin{array}{ll}\text { Samenvatting } & 177\end{array}$

The Value of Value Work: a note on valorisation 187

$\begin{array}{ll}\text { About the author } & 195\end{array}$ 


\section{ACKNOWLEDGEMENTS}

$\mathrm{Y}$ ou cannot achieve happiness alone, nor can you become a good person all by yourself. The same goes for the journey towards becoming a proper academic. My PhD brought me from Maastricht to Philadelphia, from Barcelona to Edinburgh, and from Mainz to Uppsala. Throughout this journey, I have been lucky to have encountered many inspiring, critical, enthusiastic, and empathic people. I'm grateful to many of them for providing me with good examples of how virtuous (and frivolous) academic life can be.

First of all, I am deeply grateful to my promoters, Tsjalling Swierstra and Heleen Pott. Tsjalling's sharp feedback, often happily served with a dash of irony, encouraged me to clarify my thinking, and to keep polishing my writing. Heleen's erudite and topical critiques urged me time and again to reflect more creatively on the bigger picture. When dealing with my stubbornness, both were able to combine patience, humour, and being critical - three academic virtues that I now hold dear. Thank you both for your unending support.

I'm also indebted to my colleagues and friends in Maastricht, who in some way have contributed to my thinking and doing. In no particular order: Ruud, Ruth, Tamar, Harro, Sally, Karin, Wiebe, Sjaak, René, Joke, Jacqueline, Ike, Claudia, Bernike, Cody, Annette, Lotte, Dani, Willemine, Dirk, Darryl, Koen, Gili, Mijke, Joeri, and Jan. I feel lucky to remain part of a truly interdisciplinary, critical, and informal community. Probably the best times during my PhD were spent at an old convent, at WTMC summer schools and workshops, together with fellow PhDs under the inspiring guidance of Willem and Teun. I thank the welcoming ValueS and P6 groups of TEMA at Linköping University, especially $\mathrm{CF}$, Teun, Steve, Ivanche, and Jeffrey. And a special thanks to my former dean Rein de Wilde for making it possible for me to stay in Linköping. I thank Naomi 
for designing a spot-on cover for this book, and Angel and Juliet for their excellent editing efforts.

When a PhD turned from a journey together to a journey within, I luckily enjoyed the support of friends and family. I thank Sarah, Andreas, Anna, Andrea, Gerrit-Willem, Joost, Freek, and Gijs for being there and leaving me be when needed. I am grateful to my parents, without whose endurance and persistence in the past (and present) I would not have been able to enter University altogether. Finally, thank you Dorien - we did this together, and you know what it has taken. And Leentje, I promise the next one will have more pictures. 


\section{CHAPTER 1}

\section{Brain Plasticity \\ and the Question of Moral Change}

\section{Introduction}

$\mathrm{I}^{\mathrm{n}}$ $\mathrm{n}$ the bestseller De Tien Geboden voor het Brein [Ten Commandments for the Brain] (2011), Dutch psychiatrist René Kahn asks: “We take care of our heart, muscles, bodies. Why shouldn't we do the same for our brains?" Drawing on knowledge from the neurosciences, Kahn explains how our brains are plastic and argues that we can influence its development by changing our behaviour. To take matters in our own hands - to get "the best out of your brain" - he instructs us to study hard, sleep well, stress less, make friends, exercise, play with music and art, and refrain from alcohol. Lastly, indicating some limits to our wish to control our behaviour and fate, the author ironically suggests that we should choose our parents wisely, as their genes do influence our brain's development. Kahn promises that if we follow these commandments and change our behaviour by working on our brains, we can improve ourselves.

Books like Kahn's are not easy to miss when visiting your local bookshop. In the past twenty years, neuroscience knowledge has spread from the laboratory into society. Newspapers and magazines eagerly write on how we should nurture our brains, by training through exercise, mindfulness, eating healthy 'brain food,' or living a 'brain-conscious' life. Through popular-science books, self- 
help manuals, TV programmes, apps, and neuro-gadgets such as brain games and DIY EEG headsets, neuro-advocates offer all kinds of advice and suggestions on how to best train our brains and improve our lives. The Biblical reference in the title of Kahn's bestseller -Ten Commandments for the Brain - while obviously meant as a marketing-friendly catch phrase, succinctly captures the new status the neurosciences seem to have received in our contemporary society: as an authoritative beacon we can turn to for questions of what to do and how to improve ourselves.

At the heart of this emerging brain culture (Ortega \& Vidal, 2011; Thornton, 2011) lies a specific and relatively new view of the human brain. The prevailing conception within the neurosciences for the better part of the 20th century was that the adult brain is fixed and immutable after childhood. Since the 1990s, this conception of the human brain has been replaced by a view of the adult brain as mutable through its entire lifespan. Our brains are now described as plastic, or able to functionally and structurally change due to development, experience, or injury (Malabou, 2008; Trevarthen, 1987). Brain plasticity "opens up" the brain for all kinds of interventions (Abi-Rached \& Rose, 2013), as we are invited to do something with it. The idea of a plastic, changing brain allows neuroscientists and neuro-advocates to propose all kinds of prescriptions for what to do and how to flourish - in short, it allows them to engage in ethics.

This book is about the promises of a neuro-inspired ethics. More specifically, it explores how the concept of brain plasticity is normatively used in societal practices of self-fashioning and flourishing. Questions of how to improve oneself in accordance with one's natural set-up are characteristic of the tradition of virtue ethics or good life ethics (Heller, 1988; MacIntyre, 1985; Swierstra, 2007). Ethicists in this tradition ask: How can I live a good life? How can I become a good person? How can I flourish, given the possibilities I have as human being? The aim of this book is to explore how the concept of the plastic brain is mobilized to answer these questions. How are the ethical implications of a plastic brain constructed in societal discussions about self-fashioning and flourishing? What are the consequences of reformulating the timeless question of the good life into neuroscientific terms? To answer these questions, I turn to different societal domains - practices of neurobiological self-fashioning - where the plastic brain is made valuable as a means to articulate what we can and should do in order to flourish and live well.

In this chapter, I introduce the concept of plasticity and identify the challenges it poses for understanding the ethical and societal implications of the 
neurosciences. I argue that both proponents and opponents of a plastic brain share the assumption that the proliferation of the plastic brain automatically will result in specific moral changes - for better or for worse. They thereby forgo the question of how the plastic brain is actually made valuable in specific contexts in the first place. In this dissertation, I sidestep the theoretical debate between proponents and opponents by taking a pragmatist and empirical approach towards understanding how the ethical implications of the concept of brain plasticity are enacted in practices of neurobiological self-fashioning. After I introduce my analytical framework for studying valuations of a plastic brain in these practices, I introduce three case studies in which I scrutinize neurobiological flourishing.

Throughout history, when faced with questions of what to do and how to improve our lives, we turned to counselling experts - be they philosophers, church-fathers, essayists, or spiritual gurus. As the Big Science of today, the helping hand now seems to come from the neurosciences. In a society where (technological) change is the imperative, the plastic, changing brain appears to a fitting means for addressing challenges and ideals for living well. By showing how the plastic brain is used to answer this timeless ethical question, this book aims to give a glimpse of how, in an affluent and secular society, the 'hard' and technologized sciences become imbued with the promise of bringing us evermore closer to the good on the horizon.

\section{Promises and Perils of a Plastic Brain}

Traces of the idea that our brains are not fixed or hard-wired after a certain age, but instead remain changeable throughout our adult lives, can already be found in the history of the neurosciences. Historians of (neuro)science have shown how psychologists and neuroscientists throughout the past century already considered the idea of plasticity (Abi-Rached \& Rose, 2013; Pitts-Taylor, 2016; Rubin, 2009; Teskey, 2001). William James, for example, is often seen as one of the early predecessors who thought about the brain as a plastic organ, as he stated in 1890: "organic matter, especially nervous tissue, seems endowed with a very extraordinary degree of plasticity" (Begley, 2009, p. 13; Pitts-Taylor, 2016, p. 24). Similarly, the quote famously ascribed to neuroscientist Hebb in 1949 - "neurons that fire together, wire together" - indicates forms of synaptic plasticity: changes in the strength of connections between synapses, the junctions between neurons. Syn- 
aptic plasticity was generally accepted at the first half of the $20^{\text {th }}$ century, just as the idea of critical or sensitive periods in the brain development of infants (AbiRached \& Rose, 2013; Pitts-Taylor, 2016). Still, the dominant view within the neurosciences (and beyond) in the $20^{\text {th }}$ century was the view of Santiago Ramón y Cajal, one of the founding fathers of modern neuroscience:

Once development has ended, the founts of growth and regeneration of the axons and dendrites dried up irrevocably. In the adult centers the nerve paths are something fixed, ended and immutable. Everything may die, nothing may be regenerated. It is for the science of the future to change, if possible, this harsh decree. (as cited in Begley, 2009, p. 13).

Cajal perceived this immutability of the human brain as harsh because it would mean that there is no growth in an adult brain and that the brain cells at birth are all that we would ever have - implying a firm biological limit on the quest for finding remedies for brain diseases and lesions.

Cajal hoped that future research methods and technologies would shed a different light on the static image of the brain. Indeed, with the advent of new imagining technologies such as electroencephalogram (EEG), by which electrical activity in the brain could be measured, and functional magnetic resonance imaging (fMRI), which measures changes in the level of oxygen in the blood flow of the brain, the idea of a static brain became slowly replaced from the 1990s onwards with the view of our human brain as inherently plastic. This shift was further solidified by the 1990s research of prominent neuroscientist Fred Gage, which questioned the widely-held belief that adult brains were not capable of creating new neurons by demonstrating an ongoing neurogenesis in the adult human brain (Rubin, 2009, p. 418). In a field already adapted to the concept of synaptic plasticity and to developmental plasticity in infants, the brain was now envisioned as open for structural changes. This resulted in the emergence of a strong therapeutic discourse, which promised that this novel type of plasticity could come with new remedies for brain diseases and a potential for a "self-healing brain" (Society for Neuroscience, 2007; Rubin, 2009, p. 420). As this therapeutic, promissory discourse intermingled with the scientific discourse on plasticity, it paved the way for the wide acceptance of the possibility of structural brain plasticity over fixed views of the human brain (Rubin, 2009; Rees, 2010 \& 2016). As Cajal already hoped for, the harsh decree of an unamenable brain was denounced, as our plastic brain became perceived as amenable through all kinds of therapy, drugs, and self-nourishment. The plastic 
brain became the premise for those who were interested in the implications of neuroscience for therapy and policy (Abi-Rached \& Rose, 2013). ${ }^{1}$

In the contemporary neurosciences, plasticity has become an umbrella term that encompasses mechanisms of change on different levels of neural organization in the brain: from individual synapses and neurons, to structures or networks of neurons, and eventually behaviour. ${ }^{2}$ While plasticity nowadays is widely seen as a fundamental property of the central nervous system (CNS), there remains a high level of uncertainty and disagreement within the neurosciences about the degree, quality, and scope of plasticity or about the specific cells, regions, networks, or brain functions it involves (Shaw \& McEachern, 2001; Teskey, 2001). In its most simple description, plasticity is the ability of the brain to change and be changed. The brain is said to change due to genetic developments (i.e., sensitive periods in infants), self-directed thought and action, our experiences and interactions with the environment, and injuries (Malabou, 2008, p. 20; see also West-Eberhard, 2003; Wexler, 2006). Plasticity embodies different meanings, depending in part on how it is measured and defined in research designs (Pitts-Taylor, 2016, p. 23).

This shift from an immutable, hard-wired brain to an open and changeable brain allows for conversations about the malleability of human behaviour once the epitome of debates on nature/nurture or agency/structure - to be articulated in terms of the plastic brain. The concept of the plastic brain implies a mutual shaping between the individual, her brain, and the environment: the plastic brain shapes us, and we are said to be able to shape the plastic brain through our own thoughts, actions, and interactions with the environment.

\footnotetext{
${ }^{1}$ Within the diverse field that makes up the contemporary neurosciences, brain plasticity is described through multiple notions and theories. Neuroplasticity commonly refers to the ability of the brain to functionally (degree of connectivity) and structurally (how tasks are organized in the brain) change, due to either intrinsic activities such as genetic development processes, individual experiences, and self-inflicted nourishment, or extrinsic factors such as pharmaceutical interventions or electrical brain stimulation. Synaptogenesis and synaptic pruning describe the creation and removal of whole synapses or groups of synapses that build or destroy connection between neurons, while neurogenesis refers to the creation of new neurons. Brain plasticity can be seen as part of the wider development in biology from genomics towards epigenetics, where the actualization of phenotypes in the interaction between the organism and the environment are central objects of study (cf. Papadopoulos, 2011).

2 The core epistemological problem of contemporary neuroscience is the relationship between behaviour and neural activity, the relationship between mental processes and neurobiological processes (the so-called mind/body problem, or the "explanatory gap" as Abi-Rached \& Rose (2013) call it). While many neuroscientists see the brain as the seat of human behaviour, contemporary methods (such as fMRI or EEG) are based on finding correlations, not causations.
} 
Critiques on the plastic brain as being reductionist and determinist therefore seem to have lost their critical purchase (Pitts-Taylor, 2016, p. 7): Our brain is no longer only biologically determined, as it is being shaped in relation to its environment. Hence, some scholars see plasticity as bridging the gap between nature and nurture (cf. Beaulieu, 2000; Ledoux, 2002; Mohr, 2003). Nature/nurture debates are about drawing a line between what can be changed and what should be accepted as unchangeable. They are about the (limits of) malleability of man and society. Now that nature and nurture "speak the same language" (Ledoux, 2002, p. 2) - namely the language of the amenable brain - the demarcation between the two has become blurred.

At the same time, the malleability or openness of the plastic brain is not total. Whether due to developmental programmes, self-directed nourishment through use- or activity-dependent plasticity (Ganguly \& Poo, 2013), or exogenous stimuli, plastic changes are not temporary. The term plasticity (as opposed to elasticity) implies that changes to the brain are to some extent robust, fundamental, or definitive. Like a sculpture made of stone: once it has taken form, it cannot return to its earlier state. ${ }^{3}$ This implies that plasticity is positioned in between malleability and determination, between softness and rigidity, between potentiality and actuality. Or, as the adage goes: We are our brains, but our brains are what we do with it.

The plastic brain entails that we are both objects of change and agents of change. Being both master and servant of our plastic, changing brains obscures questions of agency. In principle, the concept of brain plasticity allows for multiple views on human agency in relation to its environment. Based on conceptual analyses of plasticity by Malabou (2008) and Pitts-Taylor (2016), we can discern three (theoretical) possibilities. Agency can be seen as "inhabiting" the brain, shaping itself through processes of development or injury for example. Agency can also be attributed to the individual subject, as when our self-directed thoughts and actions are said to change our brain functioning. And agency can be located outside of the individual subject and given to culture, for example when the plastic brain is seen as susceptible to being shaped, habituated, and inscribed by social, cultural, or material influences within its environment (Pitts-Taylor, 2016, p. 22).

\footnotetext{
${ }^{3}$ Hegel, in his Phenomonology of Spirit (1807), traces the concept of plasticity to the Greek verb plassein, which means to mould or to shape, like a sculptor moulds his stone. Hegel resurrected the concept from the Greek arts (plastic arts, e.g., sculpture) and the Greek idea of self-fashioning as the moulding of character to show that we are ever capable of improving ourselves (Malabou, 2008).
} 
This indeterminate and interactionist nature of plasticity turn it into an ethically ambiguous concept. It positions us, the brain bearers, in-between malleability and determinism, and in-between our own brain functioning and our environment. The concept of the plastic brain thereby conceals a clear distinction between which aspects of ourselves or our surroundings we can consciously change, which we cannot - and who should change what. In theory, one could mobilize plasticity to address our capability and freedom for sustained and durable self-determination, but one could also use plasticity to propose changes in the way social structures or cultural factors should (or should not) shape our brain functioning. Thus, the undetermined, interactionist nature of plasticity makes it an ethically equivocal and performative concept: In principle, it affords multiple action programmes of what we can, could, and should do with our ourselves and our environment, and to what end.

\section{Normativities of a plastic brain: Beyond empowerment and control?}

As articulated by its appropriators, the promises of a plastic brain are plenty. In the past two decades, the many interventions that emerged that aim to change the brain present the plastic brain as deeply malleable. The widespread proliferation of prescription drugs for depression, $\mathrm{ADHD}$, burnout, autism, and other mental disorders is an example of plasticity-based interventions that aim to alter behavior through drug-induced neurological change.

But the promises of the plastic brain are not limited to the clinical potential of such exogenous stimuli. The therapeutic potential of the plastic brain has spilled from the clinical domain into our everyday lives. Books with titles such as The Plastic Brain - Harness the Power of Neuroplasticity for a Healthy \& Happy Brain or Neuroplasticity: This Is How to Train Your Brain for Success promise that through endogenous stimuli - self-directed plasticity through brain training we can achieve happiness, health, or success more easily and with longerlasting results. Some interventions seem to articulate new ideals, such as "brain health" or a "brain conscious lifestyle". All aspects of our lives can be made subject to the idea of brain plasticity, "for it is the life we lead that creates the brain we have" (Schwartz \& Begley, 2002, p. 286). Plasticity would give us "a remarkable built-in ability to strengthen and grow the person that you are, at any age" (Merzenich, 2013, p. 2).

An understanding of ourselves as neurologically plastic thus comes with the promise of new and better ways to improve ourselves, and maybe even new ideals to strive for. The promise is thus that knowledge of the plastic brain 
comes with certain moral changes, changes in the ways and ideas we can and should improve ourselves. This promise of neuro-induced moral change is the central topic of this dissertation. It also underlies the current debate on understanding the implications of the increasing societal import of neuroscience knowledge. In the past years, scholars from different disciplines have scrutinized the possible implications of the import of neuroscience knowledge in academia and society. We can roughly distinguish two major positions in the debate on understanding the implications of the plastic brain. Proponents and other enthusiasts - e.g., popularisers of neuroscience knowledge and some scholars in the humanities and social sciences - see in plasticity a liberating and empowering force, a new means to improve ourselves and society. In contrast, critics warn of a reiteration of an "ethic of neoliberal self-care" or a new "brainbased biomedical citizenship." (Papadopoulos, 2011; Pitts-Taylor, 2010; Rose, 2007; Abi-Rached \& Rose, 2013).

Knowledge of a plastic brain appears to be easily transferable from the neuro-lab to a variety of societal and scholarly domains. As the plastic brain is open and receptive to its environment, it can safely be used by scholars from the social sciences and humanities without facing critiques of biological or social determinism. Similar to those who see plasticity as the end to nature/nurture debates (cf. Beaulieu, 2000; Ledoux, 2002; Mohr, 2003), some scholars mobilize brain plasticity to bridge or counteract determinist and structural explanations of human behaviour, such as hard-wired sex differences and gender stereotypes (cf. Schmitz \& Höppner, 2014; Jordan-Young \& Rumiati, 2012; Vidal, 2012), technological determinism (Rotman, 2000), neo-liberalism (Malabou, 2008), or stigmas on mental illness (Martinez \& Mendoza-Denton, 2011). This reading of plasticity attributes to the plastic brain the power of liberation from (structural) constraints to our autonomy and celebrates its ability to empower our individual agency. Plasticity is interpreted here through the discourse of emancipation and empowerment, and it is seen as biology-based evidence of the potential of human adaptability and freedom.

In contrast, other scholars warn that the plastic brain has been embraced too enthusiastically. Here, the plastic brain is not hailed as an empowering ally against structural constraints to our autonomy (be they biological, technological, or economic) but rather is viewed as a reiteration or deepening of such constraints. These scholars question the apparent alignment between plasticity and dominant ethical frameworks - in the affluent West - of self-determination, healthy citizenship, embodied self-stylization, and the neo-liberal virtue of flex- 
ibility. In this view, the contemporary brain sciences should be seen as forms of power and governance, within which we are urged, through Foucauldian "technologies of the self" (e.g., psychopharmaceuticals), to care and take responsibility for properly managing our bodies (Papadopoulos, 2011; PittsTaylor, 2010; Rose, 2007; Abi-Rached \& Rose, 2013). Interventions based on brain plasticity then become yet another form of a "neoliberal ethic of personal self-care" (Pitts-Taylor, 2008). Indebted to the scholarly tradition of bio-politics (Lemke, 2011), life politics (Giddens, 1991; Dehue, 2014), and governmentality studies (Rose, 2007), scholars in this position fear that the plastic brain turns from individual opportunity into a societal obligation. The plastic brain then becomes a new a site of choice, prudence, and responsibility: "Once more, now in neural form, we are obliged to take responsibility for our biology, to manage our brains in order to bear the responsibilities of freedom" (Abi-Rached \& Rose, 2013, p. 23). ${ }^{4}$

What we see in these recurring analyses on brain plasticity is that the ethical implications of the plastic brain are either celebrated as empowering and liberating, or feared as a reiteration of the status quo - which, in a context of neoliberal bio-medicalization, would imply a further constraining or disciplining of our options to act and care for ourselves. While both positions in the debate value the plastic brain differently, they share the assumption that neuroinduced moral changes are imminent: They presuppose that the import of knowledge of neuroscience in society will automatically lead to specific normative effects and moral changes, conceptualized as either strengthening our autonomy or undermining it by instigating new forms of discipline and (social) control. Scholars in both positions run the risk of assuming and overstating the novelty and importance of the neurosciences in transforming subjectivities and practices, thereby unreflexively buying into the promise that knowledge of the brain will transform our conceptions of self, our behaviour, and ultimately what it means to be human (O'Connor et al., 2013; Pickersgill \& Van Keulen, 2011; Pickersgill, 2013; Schneider \& Woolgar, 2015). Moreover, in assuming specific

\footnotetext{
${ }^{4}$ While the earlier governmentality work of Nikolas Rose remains quite influential in studies of the societal impact of the neurosciences - especially his concepts of the "neuromolecular gaze" and "neurochemical selves" - the position he develops together with Abi-Rached in Neuro (2013) is somewhat more ambivalent. In contrast with his earlier work on neuroscience, the 'late Rose' downplays his earlier concern that our personhood would be replaced by a "brain hood," and he points to some limits of Foucauldian governmentality approaches, especially, for understanding unexpected or complex engagements with bio-medical knowledge in practice, resulting in multiple subjectivities (Rose, 2003 \& 2007; Abi-Rached \& Rose, 2013).
} 
moral changes, how such changes would come about in particular practices remains unclear. In theorizing about the societal implications of the plastic brain, both positions in the debate ignore an important question: How is the knowledge of the neurosciences made relevant and valuable in the first place? And how are these valuations made to challenge or destabilize ideas of flourishing?

It is not at all obvious that such highly technical knowledge can play a role in answering questions of what to do and how to live - let alone that it changes ideas and practices of flourishing. Several scholars have empirically scrutinized the import of neuroscience knowledge in society instead of assuming specific moral changes beforehand (cf. Pickersgill, 2011 \& 2013; O'Connor \& Joffe, 2013; O'Connor \& Nagel, 2017). For example, some of these studies explore how novelty is performed in practices of mental health care and law, revealing that knowledge of the brain seems to be made relevant in such practices not by challenging existing views on responsibility and free will but rather by aligning to existing cultural tropes, thereby perpetuating them (Choudhury et al. 2009; O'Connor et al. 2013; Pickersgill et al., 2011). In a similar vein, O'Connor \& Nagel (2017) have shown that neuro-enhancement discourse in practices of parenting children and caring for the ageing is not only taken up to reinforce individualist forms of (self-)care. In these practices, the authors show, the self and other become blurred, as parents and seniors engage with neuroscience knowledge to articulate their relationships of care towards those around them (O'Connor \& Nagel, 2017).

What these examples indicate is that it is not evident from the outset how knowledge of the brain will be made valuable in practice, nor that such valuations can be sufficiently understood through analytical frameworks of individual empowerment versus societal control. These studies show that the dissemination and appropriation of neuroscience knowledge is a deeply socially and culturally embedded process - an insight that is at the core in fields such as Science \& Technology Studies (STS), Sociology of Scientific Knowledge, and Critical Neuroscience (cf. Choudhury \& Slaby, 2012). Instead of assuming a transformative potential of the neurosciences, how neuroscience knowledge is perceived and valued as to change conceptions and practices of flourishing then precisely becomes a topic of empirical inquiry..$^{5}$ This calls for a broadening of

\footnotetext{
${ }^{5}$ The very process of rendering the plastic brain ethically valuable is thus a topic of empirical enquiry instead of a topic that requires (meta-)ethical speculation. This means that I do not engage in discussions about fact/value distinctions or the naturalistic fallacy and their mud pool of metaphysical and linguistic arguments (cf. Appiah, 2008; Hume, 1739/2000; Putnam, 2002; Quine, 1951). From
} 
the conceptual framework used for understanding the possible normative effects and possible moral changes of neuroscience in specific contexts.

This especially applies for understanding the normative usages of the plastic brain. The performativity and ethical ambiguity of the concept - its indeterminate and interactionist nature - implies that only in appropriations or usages does it become visible how boundaries are drawn between what parts of ourselves or society we can and should change, and what we should accept as unchangeable. To conceptualize these possible valuations of the plastic brain beforehand as either empowering our autonomy or disciplining it potentially limits my exploration of the ways the plastic brain in different societal contexts could be made valuable to address certain concerns, hopes, fears, and ideals of flourishing. Thus, instead of asking whether the plastic brain either limits or empowers our quest for self-determination, I ask the question: To what desirable ends is brain plasticity made valuable in practices of self-fashioning in the first place, and how are such different valuations of the plastic made to challenge or destabilize prevailing ideas of flourishing and living well in these practices?

\section{The Plastic Brain as Medicine for Living Well}

The key questions this thesis addresses are: How is the plastic brain made valuable for questions of flourishing, and how are these valuations of a braininspired good life made to (de)stabilize ideas of flourishing? To answer this question, I will explore the ways the plastic brain is made valuable in three different practices of the good life across our lifespan: parenting, working, and ageing. Before I introduce these three case studies of neurobiological flourishing, I first explicate my conceptual framework for analysing valuations of the plastic brain. Inspired by recent work in Science \& Technology Studies (STS), sociology of valuation, sociology of expectations, and pragmatist virtue ethics, I construct an analytical model for tracing the moral life of the plastic brain in society (cf. Pickersgill, 2013).

a pragmatic point of view, the blurring of the fact/value distinction when making a practical judgement is not so surprising: If we aim for a solution to the question of what to do, we need at least some view on what is the case (including what kind of human beings we are) and what the possible and desirable consequences of our actions can be. 


\section{Articulating and Coordinating the Good through Value Work}

In traditional virtue ethics, the question of what to do and why emerges when we face particular challenges that give rise to concerns. In such situations, when it becomes unclear what to do, we often turn to counsellors for advice. When normatively mobilizing the plastic brain for questions of how to do and be good, translators and popularisers of neuroscience take on this role of ethical counsellor. In the advice of what to do with the plastic brain and for what reason, the question of truthfulness of the advice is less important than its suitability to address certain concerns or problems and to bring about desirable improvements. Just like therapists, translators and popularisers of neuroscience knowledge need to tune in to the concerns, needs, hopes, and desires of their audiences to make their knowledge relevant and meaningful. To explicate how the plastic brain is used for questions of flourishing, I thus turn to the advocates of brain plasticity and trace their efforts at what I call value work.

Value work is the act and process of valuing: the attribution of value to something, and/or the assessment of something as valuable. Values can be broadly defined as 'ends-in-view' (Dewey, 1913) or as something 'desirable, good or proper' (Dussauge, Helgesson, Lee, \& Woolgar, 2015; Graeber, 2001). I use the term value work instead of valuing to denote that attributing value or assessing something as valuable requires deliberate activities: The relevance and meaning of the plastic brain, for specific audiences and for specific ends, is not given, but has to be established. I base the notion of value work on a recent body of work in the field of sociology of valuation (Dussauge et al., 2015; Helgesson \& Muniesa, 2013; Lamont, 2012), which in turn is indebted to the pragmatist view on values and valuation of Dewey (1939). A pragmatist view on values holds that values are not transcendental, fixed, or absolute entities that silently structure our behaviour. Instead, values are seen as the outcome of (social and discursive) processes of valuation, where values become enacted, articulated, ordered, and negotiated (Dussauge, Helgesson, Lee, \& Woolgar, 2015; Kjellberg \& Mallard, 2013). This shift from value as noun to value as verb - a 'flank movement' towards values, as Dewey called it - assumes an agnostic and empirical stance towards values as things that are performed (and thus can be studied) in practice (Muniesa, 2011). ${ }^{6}$

\footnotetext{
${ }^{6}$ By focusing on the process of attributing or giving value to something, one bypasses the question of whether values "really" exist, or whether they only exist in the eye of the beholder (the dualistic relationship between objectivity and subjectivity of values).
} 
Scholars who study valuation from a sociological perspective often focus on specific valuation practices, metrics, or devices in places where the assessment and ranking of values is explicitly reflected upon: TV talent shows, the assessment of peculiar economic goods, quality instruments in health care, or peerreview processes in academia, for example. I do not focus on such reflective processes of valuation-as-ranking. Instead, I see the plastic brain as a kind of valuation yardstick: It invites its users (appropriators) to articulate what we should do with it, and for what reason. It invites actors to enact, articulate, and order values - to do value work. This happens especially at moments and sites where knowledge of the plastic brain is diffused beyond the laboratory and where meaning and relevance have to be established - such as University press releases, popular science books, magazines, newspaper articles, blogs, TV shows, etc. ${ }^{7}$

Recent bodies of research in STS, particularly in the sociology of expectations and the ethics of emerging technologies, show some fruitful venues for studying such value work. For example, valuations are enacted in what some have called concern-making: addressing certain challenges or issues and translating these to (neuro)scientific knowledge, linking matters of fact to matters of concern (Dussauge, Helgesson, Lee, \& Woolgar, 2015; Kraus, 2012; Latour, 2004). As we will see, neuroscience research on ageing is often made relevant by addressing either individual issues (e.g., forgetting names) or broad societal developments (e.g., the greying society). It's through concern-making that certain diagnoses of what constitutes the problem for or obstacle to flourishing are proposed. By invoking specific challenges, certain concerns can be debunked and replaced by others, existing concerns can be reaffirmed and reiterated, or new concerns can arise. Articulating, aligning to, or debunking concerns are ways to appeal to specific audiences and persuade them to care for neuroscience knowledge.

Reflections on new science and tech often take the form of value-laden vistas of promises, hopes, and expectations about possible usages and desirabilities

\footnotetext{
${ }^{7}$ Strictly speaking, value work pervades the whole process of scientific knowledge formation, from decisions regarding funding and research priorities to the choice of theories of mind to be used, statistical models built, and behavioural concepts addressed. As I focus on the translators and popularisers of the plastic brain, I limit my examination to forms of value work on the level of dissemination and appropriation of neuroscience claims in specific value-laden contexts. In this journey of valuation, I do not assume a direct, causal relationship between sender and receiver, as is often implied in deficit-models of science communication, or notions of science communication as "knowledge transfer" (cf. Horst, 2016; Wyne, 1992).
} 
(Lucivero et al., 2011; Van Lente, 2012). Promises and expectations are generative: They guide activities, provide structure and legitimation, attract interest, and foster investment (Borup, Brown, Konrad, \& Van Lente, 2006). They form the bridge between the "inner and outer worlds of techno-scientific knowledge communities and fields" and link the technical realm of science with societal issues (Borup et al., 2006, p. 286). Promises and expectations are closely linked to concern-making: Only after addressing the problem can a solution be promised - a solution that often takes the form of action programmes or ideals. Concern-making and promissory discourse are basic features for any debate on the societal impact of modern science and technology (Brown 2003; Swierstra \& Rip, 2007; Swierstra 2015), and they provide a rich resource for studying how valuations of science come into being.

By appealing to the perceived concerns and challenges of a specific audience and linking scientific knowledge to their hopes and ideals, advocates of emerging science and technology not only attribute (moral) relevance to scientific claims but also prepare the ground for a normative appropriation of these claims. Articulating certain values and not others, or aligning the plastic brain with certain ideas of the good and contesting others, is a way to anticipate ethical appraisal and possible value conflicts. Coordination of the normative uptake, through mobilizing concerns, promises, and expectations, is an important function of value work and turns it into a form of ethical boundary work (cf. Wainwright et al., 2006). ${ }^{8}$

To explore to what extent the plastic brain in practice amounts to moral changes, I thus explicate the ways advocates are able to render the plastic brain relevant and meaningful for particular audiences, and for particular ends, through articulating values in concern-making and promissory work. I do this by tracing the trajectory of valuations of the plastic brain from its diffusion in public discourse to concrete interventions in particular contexts. This entails studying the ways neuroscientists themselves - but also science journalists, companies, social scientists, and other neuro-translators and popularisers - use the plastic brain to articulate what it means to do and be good. As studies in ethics of emerging technologies have shown, value work does not seem to happen at random: Public deliberation about emerging technosciences such as the neurosciences are often characterised by recurring patterns of moral argumen-

\footnotetext{
${ }^{8}$ Wainwright et al. (2006) coin this term, based on Gieryn's notion of boundary work, to show how ethics has become an integral part of maintaining the image of science in in the embryonic stem cell laboratory.
} 
tation (cf. Borup et al., 2006; Swierstra \& Rip, 2007). By explicating the journey of valuations of the plastic brain, I aim to discern possible recurring patterns of valuations. I thereby focus on a specific subset of valuations: those that pertain to the good life.

\section{A Pragmatist and Therapeutic Model of the Good Life}

To study valuations of the plastic brain in practice, I take a pragmatist stance towards ethics (cf. Keulartz et al., 2004). A pragmatist view on ethics does not start from abstract or absolute laws or principles but instead focusses on the acting individual in concrete practices where dilemmas and challenges occur. Dualisms such as fact versus value, objectivity versus subjectivity, or technology versus culture - often the object of metaphysical ruminations in the field of ethics - are not seen as essentialist or a priori given but are constructed in practice where they fulfil a certain function (Keulartz et al., 2004). I treat ethics as being about the concerns of everyday life, about the practical questions of what to do and how to live. Not every how-to question is ethical; I regard only those questions that deal with how to act in relation to our own and others' wellbeing as ethical (Sayer, 2011, p. 145).

As the promises of plasticity in popular culture are about new selfunderstandings and new ways to improve ourselves, I take my inspiration from good life ethics or virtue ethics. In this revived tradition of ethical thinking, the focus is not on consequences or principles but on the possibilities of individual self-realization or self-fashioning in a particular practice. Virtue ethics deals with questions such as how to become a good person, how to attain a good character, and how to flourish in a particular practice given the possibilities one has as a human being. This practical view on ethics as characterized by wellbeing or flourishing - which is not limited to individualistic virtues but can also include socially oriented virtues such as honesty, concern for others, or kindness - is in stark contrast with both rule-based and principle-based ethical theories, which search for absolute or general laws and regulations (such as Kant's categorical imperative), and consequentialist ethical theories, which emphasise consequences of actions (such as utilitarianism).

There are many different theories of virtue ethics, each with different conceptions of how we should live our lives (cf. Annas, 1993; MacIntyre, 1985; Slote, 2001; Williams, 1985). Instead of theorizing about such virtue ethics theories, or measuring up practices with a specific virtue ethics theory in hand (as is common in applied ethics), I take a practical stance, akin to practical philosophy 
or empirical ethics (cf. Mol, 2002; Pols, 2004; Swierstra, 2011): There is ample ethics in the world out there, as ethics is a pervasive yet largely implicit dimension of our everyday life. ${ }^{9,10}$ The task of the philosopher or ethicist is not to measure or correct the daily lay-ethical doings with abstract theories in hand, but rather to help make visible, articulate, and broaden such lay-ethics. I will thus not side with one particular theory of virtue ethics but instead will explore how, in particular societal practices, the plastic brain is made valuable for questions of flourishing by neuroscience translators and popularisers.

Instead of a substantial, preconceived theory of virtue ethics, I therefore need an analytical tool that is abstract enough to capture the range of views and ideals of the good life proposed across three different cases studies, yet sensitive enough to explicate the characteristics of such ideas of the good life and how they are envisioned to change existing notions of the good. To this end, I combine theories from two different pragmatist ethicists. I use the analytical model of virtue ethics and moral change of Swierstra (2011), and the concept of therapeutic argument of Nussbaum (1994), in order to distinguish some basic elements or variables that any theory of the good life needs to address.

According to Swierstra, any theory of virtue encompasses ideas about the perception of ourselves, or self-knowledge, and of the practical options we have to act, or what we can call self-stylization (or ascesis). Self-knowledge is about how we understand and know ourselves. Knowledge about ourselves can arise from experiences or from the opinions of others, but it can also come from science and technologies, such as diagnostic tests. A brain scan gives a different understanding of one's self than, say, a family history or a religious community. How we come to know and understand ourselves has implications for what we can do. Factual descriptions of what it means to be a parent, an employee, or a senior are not neutral, but are action-guiding. Understanding a scientist to be a civil servant or a truth-seeker indicates different ideas of what a good academic should be and do.

In addition to self-knowledge and practical options to act, any theory of virtue also includes some view of the world or environment in which we can flourish, including the degree of control or influence we think we have over this

\footnotetext{
${ }^{9}$ The applied ethics-view is also common in the field of neuroethics, a subfield of bioethics where scholars study the ethical and philosophical implications of neuroscience research (see for example Racine, 2010; Roskies, 2002).

${ }^{10} \mathrm{I}$ base this view on the ontological assumption that our relation to ourselves, each other, and the world around us is one of valuation (Dewey, 1956; Taylor, 1986)..
} 
world. Do we perceive the world as having a particular order, or rather as a contingent chaos? How much room for achieving the good life do we have in the environment in which we are supposed to flourish? Can we bend the environment to our will, or are we rather subject to fate? These views on the world and the environment in which we are supposed to flourish affect the practical possibilities we think we have and how we can and should operate within them. In the tradition of virtue ethics, ideas of what it means to flourish as a human being are always situated within a particular context. For example, for Aristotle this context was the political life in the polis, whereas for Epicurus it was the social life of friendship. In contrast to purely subjectivist models of virtue ethics, the analytical model of Swierstra (2011) allows me to conceptualize and deduce assumptions about the world and environment in which we are to flourish. ${ }^{11}$ The resulting model of a good life is thus conceptualized along four variables: perception and action, and self and world.

Building on Dewey's pragmatism (1954) and on philosophies of technological mediation (Ihde, 1990; Latour, 1992 \& 2005; Verbeek, 2000), Swierstra uses these basic elements to conceptualize how recent and emerging technologies might impact ideas of the good life. Emergent science and technologies - such as the neurosciences - are able to exert influence over our interpretations, our norms and values, and our established interpretations. In turn, values and norms can inform the development of science and technology (Swierstra, Van Est, \& Boenink, 2009). This mutual shaping of technoscience and morality is called techno-moral change (Swierstra et al., 2009). An iconic example is the introduction of the contraceptive pill. The pill gave women a choice over family planning and gave them autonomy over their own bodies, changing their selfunderstanding, while it also came with new (gendered) responsibilities and requirements of self-discipline. At the same time, the pill created the possibility to separate sexuality from reproduction, which paved the way for the sexual revolution in the 1960s and the subsequent emancipation of homosexuals (Keulartz et al., 2004). Other now well-studied examples that are closer to the topic of this dissertation involve the use of neuro-pharmaceuticals. The use of Ritalin and Adderall by college students to improve their cognitive abilities is not only a new practical possibility (of which its refusal can also become a moral choice) but also potentially challenges what comes to count as talent, intelligence, and

\footnotetext{
${ }^{11}$ Swierstra uses the term 'world' to refer to perceived degrees of chaos/order and fate/will. In addition to these fundamental world-making outlooks, I also include in this variable more specified views on society in which we are supposed to flourish.
} 
the skills needed for learning (Sandel, 2007). Similarly, the widespread use of anti-depressants has challenged our views on sadness and tragedy in life and on which aspects of our environment we believe to be under our influence and control (Dehue, 2008).

Emerging technosciences such as the neurosciences have the potential to challenge our perception of the world and how we act upon it. The plastic brain can, for example, destabilize or change how we perceive ourselves and how we see an issue or concern. It can also challenge the practical (im)possibilities we have for achieving the good life, including the virtues, skills, or dispositions needed to overcome a challenge. And it can (de)stabilize the underlying hierarchy of justifications and valuations we use for articulating what it means to live well. This implies that neuro-induced moral change not only reveals itself in substantial changes in (articulations of) the good but can also take the form of a change in the visibility or prioritizing of certain valuations over others, highlighting some while pushing others from view. The concept of moral change is necessarily pragmatist: In order to explore shifts in our understanding of the good, or in the hegemony of valuations, it assumes that our ideas of the good are not transcendental or fixed but instead are subject to change. Another way of putting this is to say that I empirically study possible shifts in the acceptance of certain valuations, not their acceptability in principle.

Swierstra's model allows me to analyse how the plastic brain is used to propose certain moral changes regarding the four basic elements of a good life. It also enables me to analyse how the relationship between the self and the environment is constructed in brain-based accounts of flourishing - the interactionist nature of plasticity. However, it falls somewhat short for analysing the perceived malleability of the plastic brain. As we have seen, the concept of plasticity promises to offer both a new understanding of ourselves and a new understanding of what we might become. Plasticity would explain both "the origin of the person you are, and the better, stronger person that you could be" (Merzenich, 2013, p. 14). In Swierstra's model, self-knowledge only pertains to the actual understanding of ourselves, not to the ideals of what we might become.

Nussbaum's notion of therapeutic argument can help here. Nussbaum uses the concept of therapeutic argument to describe how Hellenistic philosophers (virtue ethicists) pragmatically used philosophical reasoning as medicine for living well (Nussbaum, 1994). Nussbaum's model is an illustration of how early Greek and Roman philosophers such as Epicurus, Seneca, and the Stoics proposed (moral) changes to improve daily lives. Based on a medical model of 
ethics, the aim of a therapeutic argument is to give a diagnosis of suffering - or the lack of flourishing - and to propose practical tools to overcome these obstacles in order to attain the good life (Nussbaum, 2001; McMahon, 2003). Nussbaum's therapeutic argument consists of a diagnosis of the disease (i.e. socially taught beliefs), a norm of health or conception of flourishing, and a method or procedure to attain this ideal. Nussbaum makes a relevant distinction between diagnosis and ideal: between the obstacles that prevent us from flourishing, and an ideal of what we can become when following the philosopher's advice. In Nussbaum's model, our understanding of ourselves (self-knowledge) is thus differentiated between what we are in actuality and in what we potentially can become. While the 'medicine,' or practical tools, for Hellenistic philosophers consisted of proper philosophical reasoning, the medicine I am exploring here is not philosophical but neurological: it is the plastic brain that promises to give new knowledge and tools to attain the good life. I therefore tweak and translate Nussbaum's elements of a therapeutic argument to complement and refine Swierstra's model of virtue ethics.

Using this analytical model, I aim to explicate the value work needed to make the plastic brain relevant for questions of flourishing. As I am looking for recurrences in such valuations, I call this model a therapeutic repertoire of living well. A repertoire can be seen as a result of successful value work: When certain valuations are taken up and repeated by different advocates, they slowly become solidified, settled, and thereby more easily used by others. A therapeutic repertoire thus consists of a recurring configuration of diagnosis and ideal (selfknowledge), an action programme and the skills needed to achieve it (practical options), and views on the environment in which we are supposed to flourish (i.e., world view and societal challenges). I base the notion of therapeutic repertoire on the notion of interpretative repertoire (Mesman, 2008; Wetherell, 1998; Wetherell \& Potter, 1988). ${ }^{12}$

Combined, this results in the following model (see Figure 1). The first element is self-knowledge, which includes a diagnosis (actuality) of the obstacles that prevent us from achieving an ideal of flourishing (potentiality). To what extent does the plastic brain offer a new perception of ourselves, of who we are and who we might become? The second element consists of ideas about self-

\footnotetext{
${ }^{12}$ The concept of repertoire - etymologically, an inventory or treasury (repertorium) - is a "habitual line of argument comprised from recognisable themes, common places and tropes" (Wetherell, 1998, p. 400), which "determines what those involved view as relevant, which arguments or strategies, they feel, matter" (Mesman, 2008, p. 49).
} 
stylization, or askesis: an action programme or norm that aims to guide behaviour - what we can do to overcome the diagnosis and achieve the ideal - including the skills and virtues needed to flourish. What kind of practical options for selffashioning does the plastic brain afford us? And how do these new options challenge the skills and virtues needed to flourish? The third element consists of a perception of the environment in which we are supposed to flourish (including social beliefs and societal expectations about this environment), while the fourth element captures the degree of grip or influence we think we have over this environment. What kind of world or environment is implied by the selfknowledge and action programmes of flourishing? How orderly or chaotic do we perceive this world to be, and how much control and room does that imply for us to achieve the good life? These four elements allow me to map the ways the plastic brain is made valuable in practices of flourishing, and to discern for what kinds of problems, prescriptions, and ideals the plastic brain is normatively used - in short, it enables me to analyse how the ethical implications of the plastic brain for achieving the good life are constructed in practice.

\begin{tabular}{lll}
\hline & Changing perceptions & Changing practical options \\
\hline Self & $\begin{array}{l}\text { (1) Self-knowledge: diagnosis and ideals } \\
\text { of flourishing }\end{array}$ & $\begin{array}{l}\text { (2) Self-stylization (askesis): action } \\
\text { programmes, skills, and virtues }\end{array}$ \\
Environment & $\begin{array}{l}\text { (3) Societal beliefs about the environment } \\
\text { in which we flourish (incl. societal } \\
\text { developments) }\end{array}$ & $\begin{array}{l}\text { (4) Degrees of grip or control we think } \\
\text { we have over the environment } \\
\text { (fate/will) }\end{array}$ \\
\hline
\end{tabular}

Figure 1: Model of a therapeutic repertoire of living well showing the relations amongst its four key variables.

\section{Three Cases of a Brain-inspired Good Life}

In the chapters that follow, I will demonstrate the normative usages and the construction of ethical implications of brain plasticity in three societal domains: parenting, working, and ageing. In these value-laden contexts of neurobiological flourishing, a particular manifestation of the plastic brain has emerged in recent years: the teenage brain in the context of parenting; the adult, stressedout brain in the context of work; and the ageing brain in the context of self-care. 
These cases were selected after an initial LexisNexis analysis on the issues connected to brain plasticity in Dutch public discourse. ${ }^{13}$

Case selection was guided by three theoretical considerations. First, the cases each represent a specific part of a good life across the lifespan, where there is (continuous) discussion about what it means to do and be good. Each case addresses particular (societal) challenges for which the plastic brain is heralded as a solution or means to deal with these concerns. Second, each case study seems to feature a particular manifestation of the plastic brain. These plastic brains differ in their emphasis on a specific configuration of the ethical ambiguity of plasticity: agency (who is changing the brain) is attributed either to the environment (first case), the individual (second case), or the brain itself (third case).

Third, cases were selected in which the plastic brain was mobilized to propose mundane or soft interventions. This means I selected cases that feature forms of endogenous, activity-based plasticity - forms of neurobiological selffashioning, or what we can call neuroascesis (Ortega, 2011) - rather than exogenous, invasive, medical technologies such as pharmaceuticals. ${ }^{14}$ There is already ample research done on the ways pharmaceutical interventions challenge and destabilize certain practices (cf. Dehue, 2008; Dehue, 2014; Furedi, 2004; Horwitz \& Wakefield, 2007; Rose, 2007; Abi-Rached \& Rose, 2013). Focusing on pharmaceutical interventions when exploring possible changes in our ideas of the good life might be a bit of a red herring, as it is more likely that engagement with neuroscience knowledge penetrates everyday life through less extreme and costly practices (Kraus, 2012; O'Connor \& Joffe, 2013). ${ }^{15}$ At the same time, these 'soft' technologies can make visible the possible moral changes in daily life inflicted by 'hard' technologies; the interventions under study are based on research that is itself the outcome of a technologized understanding of human behaviour (i.e., through fMRI scanning techniques in highly technologized research environments). The three cases show how brain plasticity is made valuable for common and everyday concerns and challenges in different phases of

\footnotetext{
${ }^{13}$ LexisNexis Academic is a news media database that contains the archives of over 10,000 newspapers, magazines, and other published (Dutch and international) media.

${ }_{14}$ There are many more societal domains where the plastic brain also appears as an emerging framework for understanding topical concerns such as nutrition and baby-brains; education and sexed brains or ADHD brains; or law and criminal brains.

${ }^{15}$ While these three cases span three meaningful constituents of our adult life, it should be clear that this selection is not exhaustive for the appropriation of the plastic brain in society and thus puts some limits to my reflection on the ways the plastic brain is made valuable for questions of flourishing.
} 
our good life, across the lifespan. I briefly introduce each case, then I present my empirical material and address some methodological limitations.

In chapter two, I explore how the notion of the teenage brain is mobilized in contexts of pedagogy and parenting to tell parents how to parent their adolescent children. I explore the valuations of the teenage brain by following the popularizing work of Dutch neuropsychologist Eveline Crone, who played a leading role in establishing the concept of the teenage brain in Dutch public discourse as a widely used vocabulary to address adolescent behaviour. The idea that the teenage brain is not finished and is therefore impressible to environmental stimuli took flight in parenting magazines and pedagogical circles as basis for a variety of prescriptions to parents of what they should do with their teenagers, and why. To answer the question whether the teenage brain challenges ideas of good parenting, I put the plastic, teenage brain in the historical context of science-based parenting advice, which started in the 1950s with books such as Dr. Spock (cf. Depaepe, Simon, \& Van Gorp, 2005; Wubs, 2004). As we will see, the teenage brain as parenting advice offers parents different (and at times conflicting) prescriptions for dealing with their adolescents.

In chapter three, I analyse a different kind of plastic brain: the adult, stressed-out brain on the contemporary work floor. Here, the changing brain is not a given, as in the case of the teenage brain, but becomes a task for modern employees: Agency to change the brain is attributed to the individual subject. I focus on the intervention of mindfulness. Training programmes and popular management books invoke knowledge of the plastic brain to diagnose problems on the work floor and to herald the benefits of meditation and mindfulness. In these programmes, authors invite readers and participants to take control of, and to change, their brain functioning in exchange for new ways to increase productivity and achieve happiness and well-being at work. The mindful brain is presented as a particularly plastic brain, a brain able to withstand and adapt to changes on the work floor. Instead of following a specific actor, as in the case of the teenage brain, here I trace valuations of the mindful brain from a group of neuroscientists to specific mindfulness programmes for managers and employees. As I will argue, advocates use the mindful to cater to different demands for being a good employee in the "new spirit of capitalism" (cf. Boltanski \& Chiapello, 2005; Davies, 2015). Work in mindfulness programmes becomes a kind of vocation, in which flourishing depends on a disciplined lifestyle. Compared to earlier ideas of work in the history of management and 
work, the ideal employee in mindfulness programmes combines emotional selfcontrol with social self-fashioning.

In chapter four, I explore the valuations surrounding the ageing brain in the context of self-care. In contrast to the long-held idea that our brains passively deteriorate when getting older, losing neurons along the way, since the 2000s the ageing brain has been presented as a plastic brain that is characterized not only by decline and decay but also as an active, adapting brain still capable of neurogenesis and change. Here, agency for change is located in the brain itself. This newly found plasticity of the ageing brain invites actors to articulate a variety of action programmes targeting how seniors should cope with these brain changes. I follow the trajectory of the ageing brain through the public controversy about a specific and popular intervention: brain games for the elderly. These commercial applications of neuroscience knowledge, which form a billion-dollar industry, sparked a scholarly and public controversy. Brain games for seniors appear to function as an ethical yardstick used by advocates in advice literature to propose alternative action programmes and ideals of ageing to seniors. As I will argue, advocates use the ageing brain to reiterate and tap into different views of successful ageing, reflecting ambiguous philosophical views on old age throughout history.

In the concluding chapter five, I reflect on the ways popularisers and translators have made knowledge of the plastic brain valuable in these three contexts of neurobiological self-fashioning. Whereas the empirical chapters exemplify the specificity of valuing plasticity and its moral changes for a particular context, here I aim to draw some similarities in valuations of the plastic brain and to reflect on how the ethical ambiguity of the concept played out in the different cases. I return to the debate on understanding the ethical implications of the neurosciences in order to show how the approach of value work contributes to understanding of the ethical implications of neuroscientific knowledge for our contemporary society.

\section{Studying Popular Neuroscience in Discursive Practices}

To study valuations of the plastic brain in the three cases, I use a variety of methods and sources. The empirical material spans both the dissemination of specific neuro-research on plastic brains in a diverse range of media in Dutch popular culture as well as the appropriation of that research by different and context-specific actors. Sources include research articles, press releases by universities, interviews with neuroscientists, policy reports, mass media (newspa- 
per articles, magazines), genre-specific media (i.e., parenting magazines, management magazines, magazines for seniors), digital media (blogs, online forums, and official websites of neuroscientists and companies), popular science works (books and popular science magazines), self-reports, and scholarly critiques. This material focuses on public debates and contexts of application in the Netherlands between the time period of 2000-2015, though non-Dutch material is included when it is translated to or appropriated in Dutch debates and contexts. ${ }^{16}$ I only included texts in which the plastic brain features as a significant and substantial part of the author's argument; texts in which knowledge of the neurosciences only plays a marginal role in the argumentation are excluded. ${ }^{17}$ Texts in which knowledge of the (plastic) brain is only used in a clinical context, without general prescription for the daily lives of parents, employees or seniors) were excluded as well. I focus foremost on the written word; some video fragments are included (lectures, presentations and interviews), but overall, visual imagery is excluded from analysis. I selected the most widely read popular-science works using LexisNexis and bestselling lists of branch organizations (debestseller60.nl) and consumer websites (bol.com and managementboek.nl, for example). A more specific overview of the selection of sources for each case is presented in each of the case study chapters.

The majority of my research material is comprised of advice literature, including popular neuroscience appropriations in self-help literature and expository works (Mellor, 2003) - works that aim to translate recent insights from a particular discipline (in this case, a particular subdiscipline in the neurosciences) to different audiences. Expository works and advice literature are the nexus between neuroscience and society. In these materials, authors articulate and contemporary concerns; discuss practical, everyday problems, dilemmas, and difficulties; and mobilize recent insights from science to offer advice on what to do with them (cf. Fahnestock, 1998; McGee, 2005; Mellor, 2003; Turney, 2008). While such popular neuroscience is often dismissed as "neurohype," or over-inflated interpretations of science (Illes et al., 2010; Tallis, 2009; Quart, 2012; Caufield, 2010; Grubs, 2016), these dismissive labels ignore how public debates and popular discourse on

\footnotetext{
${ }^{16}$ When using quotes from Dutch sources, I use the author's own English translation wherever one is available; otherwise, the translations are my own.

${ }^{17}$ Using the snowball technique, I included as much relevant material as possible until the moment of saturation occurred (when no new valuations and/or therapeutic arguments seemed to emerge). However, to be clear, the goal of analysing these sources is not to quantitatively count and weigh every possible valuation of plasticity; it is to provide insight into the multiple ways the plastic brain is made valuable within these contexts.
} 
emerging science and technologies can provide a rich resource of valuations and ethical deliberations (see also Van de Werff, Slatman, \& Swierstra, 2016). ${ }^{18}$ Moreover, as the neurosciences span a huge variety of highly specialized subfields, this same popular-science work is often an extension of professional scientific debates and, moreover, are frequently used as legitimate sources by scholars within different subfields in the neurosciences, as well as by scholars in the humanities and social sciences (Cassidy, 2006; Littlefield \& Johnson, 2012). As such, popularscience works are part and parcel of the success of the neuroscience endeavour itself (cf. Heinemann, 2012; Johnson \& Littlefield, 2012; Pickersgill, 2011).

Given the diversity of empirical settings and disciplines in which the plastic brain intervenes in the three cases (i.e., pedagogy, management, gerontology), an interdisciplinary perspective is called for. Building upon theories and methods drawn from both STS and practical philosophy, I use a combination of methods, including both historical and contemporary document analysis as well as elements from argument analysis (i.e., Toulmin's model of argumentation [Toulmin, 1958]), conceptual analysis, and (critical) discourse analysis (cf. Fairclough, 1995; Hajer, 2006), to devise a tool for "normative analysis." This tool is intended to tease out the explicit and implicit valuations in texts about the plastic brain. The goal of my normative analysis is to deconstruct public deliberation on neuroscience along normative lines, recognizing patterns of valuations (i.e., therapeutic repertoires), analysing how factual and normative statements intertwine, and reflecting on the conditions of possibility of such argumentations. I manually labelled and coded valuations and normative instances of plasticity in each text; these were partly deduced from the elements of a therapeutic argument (challenge/concern, diagnosis, action programme, ideal) and partly a result of the presence of specific themes and tropes that eventually emerged from the material. I looked not only at the content of individual texts but also at the relationship of valuations between different (kinds of) texts in order to discern recurring therapeutic repertoires as well as changes in valuations of the plastic brain in the public debate over time.

The now-already-two-decades-long 'turn' to practice and empirics in contemporary theory urges me to explicate what I mean when I employ the term

\footnotetext{
${ }^{18}$ I will thus not engage in philosophy of science debates about the validity or truthfulness of (popular) neuroscience knowledge claims, un-masking their hidden epistemological and ontological assumptions regarding the translation of (concepts of) human behaviour into measurable brain states, the implied theory of mind (i.e. relation between brain/body/mind), or other conceptual difficulties that are characteristic for contemporary neuroscience research (cf. Dumit, 2004; Hagner, 2009; Rose, 1997; Rose, 2005; Schirmann, 2013). While such critiques remain relevant for understanding the scope and depth of the contemporary neuroscience endeavour, these fall beyond the scope of this book.
} 
practice in this dissertation. Contemporary scholars can use the term to mean or emphasize different aspects of human activity: that which is embodied, materially interwoven, and/or organized around shared understandings (Schatzki et al., 2001, p. 12). I focus not on the materiality of a practice, nor on the situated embodiedness of individual experiences. Rather, I focus on the constructed, shared meaning of human activity. Following the virtue ethicist MacIntyre (1997), I regard a practice as a socially established human activity characterized by certain goods that are internal to it. How the plastic brain is used to construct the shared valuations of the internal goods of practices such as parenting, working, or ageing is precisely the topic of this thesis. I thus refer to practice as the discursive activity of value work: how the plastic brain is used to articulate the shared (internal) goods of a socially established activity.

Furthermore, I study the three cases as discursive practices, which means first and foremost that I focus on analysing written documents about these practices. Instead of providing rich descriptions of situated, embodied, and lived experiences of parents with their teenagers, or experienced anxiety of seniors, I chose to focus on the broader context in which such concerns and valuations of these practices are discussed and deliberated upon. There is a practical and strategic reason for this. As this thesis aims to shed light on the ways values and norms play a role in the societal trajectory of neuroscience knowledge, it necessitates a broad scope (of three different cases of the plastic brain in societal contexts). More importantly, advice literature and expository works are places where contemporary concerns, challenges, and ideals become articulated. As historians of science know all too well, advice literature and expository works present a timely window to the moral and/or scholarly climate of the day (cf. Ortega, 2011). Authors of advice literature and popular science are in the business of selling their ideas. One could explain this as implying that their articulation of the problem and solution are not necessarily shared with the lived experiences of the subjects they describe (i.e., parents, employees, or seniors). For this thesis, it is precisely because these authors construct themselves as ethical counsellors by tuning in to their audiences that their work is highly suitable for studying how neuroscience knowledge is made valuable. ${ }^{19}$ It allows me to show how the plastic brain is used as a resource for public, ethical deliberation.

\footnotetext{
${ }^{19}$ To be clear: I do not engage in quantitative reception research, which implies that my analysis rests on the assumption that bestselling authors apparently are able to strike a chord with their target audiences and that their works have some effect on those readers.
} 


\section{CHAPTER 2}

\section{Acting as a Good External Frontal Lobe: parenting teenage brains ${ }^{20}$}

\section{Introduction}

$\prod$ eenagers. Obnoxious little creatures, if you take the word of the numerous of parents and parenting experts. Teenagers are said to be stubborn, lazy, egocentric, bold, unruly, unreasonable, irresponsible, impulsive, careless, risky, emotional, and irrational - and yes, they smell. Teenagers are seen as problematic, especially for their parents. Parents seem unsure of what to do - insecure, anxious, even desperate - finding themselves in a state of shock when their children enter puberty. Why don't they listen anymore? How can we make sure they are able to resist alcohol, or prevent them from picking up bad habits from their awful friends? How much freedom should we give them? How can we be good parents for these adults-to-be?

In the past ten years, a promising explanation to account for adolescent behaviour has fuelled enthusiasm amongst parents, educators, policymakers, and pedagogical experts: the teenage brain. The teenage brain tells the complicated story of decreasing grey cells, pruning, and a brain as work-in-progress: the teenage brain is presented as extraordinarily plastic and impressible to environmen-

${ }^{20}$ An earlier and shortened version of this chapter appeared in The Human Sciences after the Decade of the Brain (Hildt \& Leefmann, 2017). 
tal stimuli. Specific brain regions gradually take on specialized functions until adolescents reach the age of about 25. The cognitive and the emotional functions of the teenage brain are thus said to be 'out of balance,' as especially the prefrontal cortex (PFC) of the adolescent brain is considered to be in a state of ripening or maturation. As a dire consequence, the unbalanced and immature prefrontal cortex leaves our future adults in a perpetual state of impulsiveness, irrationality, and riskiness, but also one of increased sociality and creativity. The coming of age of the adolescent brain is a technical tale of wonder, opportunities, and risks.

In this chapter, I explore how the teenage brain emerged as a relevant and popular way to address issues of good parenting. In recent years, the teenage brain has been eagerly adopted in the traditionally 'soft' fields of pedagogy and developmental psychology. Pedagogues, parenting experts, and family coaches have increasingly based their parental suggestions on neuroscience knowledge of adolescent brain development. Contrary to the belief of sceptical voices, who fear that this neurobiological turn in parenting would leave parents defenceless, the topic of adolescent brain development appears to come with specific prescriptions, actions programmes, responsibilities, and courses of action. Since the prefrontal cortex of teenagers is still in development, parents are urged to complement or take over its functions - to act as an external prefrontal cortex of their adolescents' brains.

In this chapter I will address the following questions: How is the concept of the teenage brain used to tell parents what they should and should not do with their adolescents? Does the teenage brain as parenting advice challenge existing ideas of good parenting? And what does it really mean to be a good external frontal lobe? Talking about prefrontal cortices, pruning, and grey matter, it's not at all obvious that such a scientific and technical vocabulary can play an important role in helping parents answer the timeless question of how to be a good parent. Thus another important question is: How is the concept of the teenage brain, with all its scientific technicalities, made valuable for parents in the first place? And what does its use tell us about parenting in our contemporary society?

To answer these questions, I follow the journey of the work of internationally renowned Dutch neuropsychologist Eveline Crone. Crone, with her two popular-science books Het Puberende Brein [The Teenage Brain] (2008) and Het Sociale Brein van de Puber [The Social Brain of the Teenager] (2012), played a key role in establishing the concept of the teenage brain as a common vocabulary to 
explain adolescent behaviour in Dutch public discourse. ${ }^{21} \mathrm{I}$ first zoom in on the efforts Crone undertakes to make her work relevant for parents, through concern-making, raising expectations, making promises, and trying to coordinate the normative uptake of her work. I then zoom out and show how the teenage brain is made valuable by pedagogues and parenting experts to discuss adolescent behaviour and parenting issues. ${ }^{22}$ I show that the teenage brain enables parenting experts to justify and combine different and sometimes conflicting parental norms and ideals of good parenting. Thereby resulting in two distinct therapeutic repertoires of parenting well for a teenage brain.

There is a long tradition of talking about what to do with unruly youths, from Aristotle to the famous ideas of G. Stanley Hall. Since the rise of developmental psychology at the turn of the $20^{\text {th }}$ century, parenting experts emerged, and parenting has become more and more professionalized. The teenage brain as parenting advice can be seen as the latest development in this 'evidencebased' tradition of scientific parenting. Advocates promise that knowledge of adolescent brain development gives a new understanding of the 'typical' behaviour of teenagers, and that it gives parents new ways to deal with their adolescents. To question these claims of neuro-moral change, I put Crone's research on adolescent brain development in the tradition of science-based parenting

\footnotetext{
${ }^{21}$ Crone is well-known internationally for her work on adolescent brain development, and her ideas do not stand alone (cf. Casey, Jones, \& Hare, 2008; Blakemore, 2008). And while her popular science works resemble claims made in other internationally popular books on adolescent brains (cf. Jensen \& Nutt, 2015; Strauch, 2003), my focus on a single scholar has of course some methodological limitations, especially regarding the external validity of my argument. While finishing this chapter, an English translation of Crone's popular work emerged (Crone, 2017).

${ }^{22}$ As I explained in the introductory chapter, my body of empirical material spans a variety of sources. For this case study, I conducted a search of the LexisNexis news database for Dutch media coverage of the teenage brain, between the period of 2000 and 2013. A key-word search of the words 'puberbrein,' 'puber + brein,' 'puber + hersenen,' 'adolescent + brein,' and 'adolescent + hersenen,' resulted in over 500 articles in national newspapers (Volkskrant, Trouw, NRC Handelsblad, Parool, Algemeen Dagblad, Telegraaf). Duplicated articles (a result of re-printing articles in similar media outlets) were removed. From this sample, I excluded articles that discuss the teenage brain in relation to clinical diagnoses (e.g., ADHD in educational settings for example), and I selected only those articles that addressed the target audience of parents and/or that discussed issues of upbringing. This resulted in 220 relevant articles. These include bestselling newspapers with a political signature from right to left. Sources also include disciplinary magazines (e.g., Psychologie Magazine), popular (lifestyle) magazines (e.g., Viva, Leef!, HP de Tijd), parenting magazines (e.g., J/M Ouder, KidsWeek), online blogs and forums of parenting websites (e.g., www.mammaweetalles.nl, www.ouders.nl, www.jmouders.nl), and presentations and workshops for parents by local governmental portals for parental information (Centrum voor Jeugd en Gezin). Finally, I included two parenting books based on the teenage brain; one was issued by a consultancy bureau focused on youth culture (Nelis \& Van Sark, 2012) and the other was written by self-declared parenting experts (Van der Wal \& Dijkgraaf, 2013).
} 
advice regarding adolescents in order to compare the emerging idea of good parenting as external frontal lobe with these historical ideas of good parenting.

For millennia, youth have been critically assessed by older generations who often fear a shift in values and norms - usually for the worse. Studying the normative use of the teenage brain in the context of parenting shows not only how neuroscience knowledge is made valuable for parents and pedagogues but also which hopes, fears, expectations, and ideals we have for tomorrow's adults. Adolescents are a symbol for a future that needs to be understood and tamed - and the teenage brain is the means by which this question is addressed in our contemporary society.

\section{A Brief History of Parenting and Adolescence}

The question of how to deal with youths has been a concern for adults throughout history. We can find authors talk about the problems of 'annoying' youths already with the Greeks. Their concerns were mostly framed in terms of (moral) education as they questioned how to turn adolescents into virtuous citizens who respected their parents and elders. Parents (read: fathers), the "author[s] of their [children's] being" (Aristotle, Nicomachean Ethics, VIII.7, 1158b14), were expected to discipline them. Aristotle saw children as similar to animals: Both were ruled by the impulsive pursuit of pleasure (Arnett, 2013, p. 5). Youths in particular were in need of disciplining as "they have exalted notions, because they have not yet been humbled by life or learnt its necessary limitations . . . . Their lives are regulated more by moral feeling than by reasoning - all their mistakes are in the direction of doing things excessively and vehemently" (Aristotle, Rhetoric, II12). Both Plato and Aristotle regarded the time period between fourteen years and twenty-one years as a stage in life characterized by the struggle between the passions and the slow establishment of reason. Until the age of twenty-one, the passions would remain in charge and become even more problematic, as sexual desires developed (Arnett, 2013, p. 5).

While the Greeks reflected on youth as having particular characteristics, the idea of childhood as a distinct and separate stage of (biological) life only emerged around the time of the Enlightenment and the subsequent Romantic era. As the famous historical study of childhood historian Ariès (1962) suggests, 
before 1700 , children were mostly seen as mini-adults ${ }^{23}$ and were not seen as having a particular personality; Montaigne remarked at the time that children had "neither mental activities nor recognizable bodily shape" (Montaigne, in: Ariès, 1962, p. 39). Influenced by the ideas of John Locke - particularly the idea that we are all born with a mind like a 'blank slate' - Rousseau, in his famous novel Émile (1762), was one of the first modern philosophers to articulate a more substantial and Romantic view of childhood as a distinct phase in life. For Rousseau, childhood was characterized by innocence but also by rising passions and "tumultuous change" (Arnett, 2013, p. 157). As industrialization continued, childhood increasingly became a topic of interest for philosophers and other scholars, particularly in the context of education (notable and influential examples are the philosopher Dewey and the Swiss pedagogue Pestalozzi).

However, the phenomenon of adolescence as we know it today only came to full fruition at the end of the $19^{\text {th }}$ century and the beginning of the $20^{\text {th }}$ century. Adolescence - coming from the Latin adolescere, meaning 'growing up' - as scholarly subject was introduced with the voluminous publications of American psychologist Granville Stanley Hall in 1904: Adolescence: Its Psychology and Its relations to Physiology, Anthropology, Sociology, Sex, Crime, Religion and Education. Based on Lamarckian evolutionary theory, questionnaires, surveys, and even early brain studies, Hall frames the period between childhood and adulthood as a natural stage in life - one with its own biological and behavioural characteristics. Hall famously described this period as 'Storm and Stress,' a name that captures the different emotional and behavioural changes adolescents inevitably face before entering the more stable equilibrium of adulthood (Hall, 1904; Arnett, 2006). Though Hall treats a wide variety of topics and issues in his 1,300-page volumes, his perspective on adolescence echoes Plato's and Aristotle's view. On the one hand, because they possess a passion, imagination, and vitality that adults no longer have, adolescents lie at "the apex of human development" (Lesko, 2001, p. 46; Spacks, 1982, p. 231). On the other hand, they are 'becoming,' unfinished, and sexually charged beings who are in peril, a kind of 'primitives' who need to develop character and responsibility (Lesko, 2001, p. 49; Spacks, 1982, p. 231).

Hall's idea of adolescence as a specific (biological) phase of human development became hugely influential. Less well known are his pedagogical imperatives, which stressed disciplinary and instructional techniques and were set in

\footnotetext{
${ }^{23}$ Ariès famous study Centuries of Childhood: a Social History of Family Life (1962) remains an influential work in histories of childhood, despite the still on-going controversy surrounding the precise concept of childhood in medieval times (see for example: Orme, 2003).
} 
contrast to raising by tradition or neglect (Lesko, 2001, p. 88). After Hall, developmental psychology became closely linked with pedagogy (Wesseling, 2002, p. 152). With the scientization of adolescence (and childhood in general) came a scientization of pedagogy and the emergence of professional parenting experts. As the historians Depaepe (2003) and Wubs (2004) and the literature scholar Wesseling (2002) show, science-based advice from parenting experts over the past century - consisting of advice regarding parenting goals, ideals, and methods - not only reflect societal developments such as industrialization, modernization and individualization but also are closely linked to changes in theories, methods, and practices of the scholarly discipline of (developmental) psychology. ${ }^{24}$ Throughout history, parenting ideals for youth were mostly articulated in terms of (moral) character building, turning adolescents into virtuous citizens. Influenced by theories in developmental psychology, this ideal of parenting changed in the course of the $20^{\text {th }}$ century.

After the period characterized by normative pedagogy, during which different religious and ideological views (the Dutch pillarization of society) defined parenting goals (Depaepe, 2005; Wubs, 2004), the behaviourist approach towards parenting was succeeded by a psychoanalytic perspective, with the famous Dr. Spock as the most well-known proponent (cf. Spock, 1946). Prior to the 1970s, Dutch parenting advice mainly revolved around character development and the societal responsibility of parents to make sure their children became responsible and virtuous citizens, represented in the well-known and archaic Dutch formulation of tranquility, cleanliness, and regularity ('rust, reinheid en regelmaat') (Wubs, 2003, p. 210). From the 1970s onwards, parenting goals became increasingly formulated in terms of developmental psychology. Children's self-development and (emotional) well-being were no longer viewed as healthy contributors toward the development of a normative goal (such as a virtuous or disciplined citizen) but became parenting goals in and of themselves (Wubs, 2004, p. 216). Parenting experts focused on practical methods and techniques of parenting instead of explicating parenting ideals. An example is the notion of parenting style, which became popular in the social sciences by the

\footnotetext{
${ }^{24}$ The histories of both pedagogy and developmental psychology are complex and fluid. For reasons of clarity and space, I limit my historical sketch in this chapter to ideas of science-based parenting in the $20^{\text {th }}$ century in the Netherlands, with an emphasis on the period after WWII. Based on several historical overviews and handbooks, I focus on parenting advice in general, and specify or extrapolate it to adolescents. I do not differentiate here for histories of class (working class versus elite), gender or race, though the role of advice has obviously differed for these societal groups (see for example Lesko, 2001).
} 
1980s and 1990s (e.g. Baumrind, 1971). During this time, developmental psychology remained a strong source of authority for parenting experts, though a new form of evidence emerged as well: genetics. An example is Judith Harris' The Nurture Assumption (1998), in which she argued that genetic factors and peers are far more influential for the development of the child than parents; the role of parents and of parenting should be seen as minimal (Harris, 1998). The teenage brain can be seen as the latest evidence in this 'biological turn' of parenting advice, but with quite different normative inferences, as I will show.

This short sketch of the history of the scientization of parenting in the $20^{\text {th }}$ century shows that it took many forms: from the Victorian goal of a disciplined and virtuous citizen, to a Freudian focus on emotional well-being and affective parent-child harmonies, towards the modern ideal of self-development through negotiation and a more equal parent-child relationship. Since the Greeks and on throughout the $18^{\text {th }}, 19^{\text {th }}$, and $20^{\text {th }}$ centuries, adolescents were seen as facing a struggle between reason and the passions, between rationality and emotion, their energy and vitality celebrated by adults at the same time that their conflictual primitivism was condemned and disciplined. As we will see, the teenage brain is a continuation of this cultural tradition which views the adolescent as the latest episode in the modern scientization of parenting advice. Questions that thus emerge are: How is adolescent behaviour conceptualized in terms of the brain? And how is knowledge about the developing adolescent brain made relevant for parents and pedagogues?

\section{Adolescent Brain Development and the Maturing Prefrontal Cortex Thesis}

Research on the brains of adolescents is fairly new. Actual research on human brains in puberty and adolescence started only in the 1970s and 1980s. These studies were rare though, because post-mortem brains of adolescents were difficult to obtain (Choudhury, 2010). Things changed with the rise of brain scanning techniques. In 1999, Jay N. Giedd, a child and adolescent psychiatrist at the National Institute of Mental Health (U.K.), published the first longitudinal brain imaging study on the brains of adolescents in Nature Neuroscience. Giedd, Blumenthal, Jeffries, Castellanos, Liu, Zijdenbos . . \& \& Rapoport (1999) showed that there are different peak levels of gray matter in different brain regions during different developmental stages of the adolescent brain. This indicates that 
besides the first three years after birth, a then well-known critical phase of brain development, a second critical development stage could be distinguished. This would mean that adolescents' brains are not finished or fixed yet, but still developing: adolescent brains are plastic. The much-quoted article of Giedd et al. became a landmark in studies of the adolescent brain.

\section{Crone's Teenage Brain}

In the Netherlands, knowledge about adolescent brain development has been brought to the general public mainly through the works of Eveline Crone, professor of Neurocognitive Developmental Psychology and head of the Brain and Development Research Centre at Leiden University, the Netherlands. In Het Puberende Brein (2008) and Het Sociale Brein van de Puber (2012), both popularscience bestsellers (the 2008 volume having sold over 70,000 copies), Crone describes the process of brain development in adolescents by focusing on cognition, emotion, sociality, and creativity. Based on existing animal and lab studies, patient studies of brain injuries, and her own functional magnetic resonance imaging (fMRI) studies on adolescents in her Brain \& Development Lab in Leiden, Crone explains how the onset of puberty comes with increased release of gonadotropin-releasing hormone $(\mathrm{GnRH})$, and how this hormone interacts with the organization of brain functions. During the brain development of adolescents, specific brain regions grow into specialized functions by a process of pruning: a subsequent decrease after a sudden increase of grey cells. The speed of this process of pruning differs for specific brain structures, which would determine when teenagers acquire specific skills during the different stages of adolescence (Crone, 2008, p. 4). This ripening would make the teenage brain particularly susceptible and impressible to environmental stimuli.

The fast ripening of some areas combined with a slower ripening of others is said to explain many typical teenage behaviours. Because of a slow ripening of the lateral frontal cortex, teenagers are said to have problems with memorization, processing information, inhibition of behaviour, and planning. In contrast to its still-slowly-increasing cognitive power, the emotional functions of the teenage brain (seen as located in the amygdala and basal ganglia) are said to be oversensitive and hyperactive. According to Crone, teenagers, especially sensitive to the possibility of rewards, are often driven by "the pleasure area in the brain," the nucleus accumbens (Crone, 2008, p. 113). The relationship between the 'cognitive brain' and the 'emotional brain' is thus seen by Crone as one of competition and strife, resulting in an 'imbalance' that is characteristic of adolescent behaviour. 


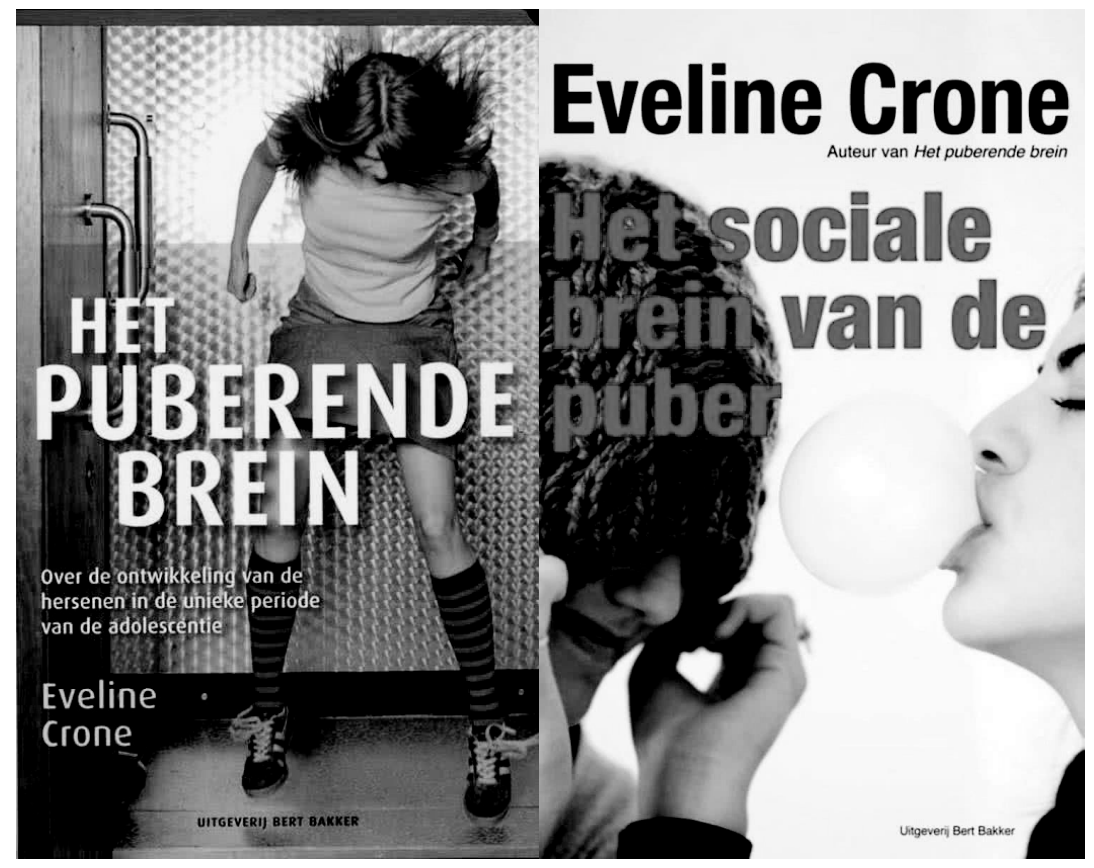

Figure 2: Eveline Crone's two bestselling popular-science books on the teenage brain, which established the notion of the teenage brain in public discourse in the Netherlands.

The slower ripening of cognitive skills, combined with an overactive emotional system, form the basis of the maturing prefrontal cortex thesis (PFC thesis). The PFC thesis accounts not only for the imbalance between cognitive and emotional brain functions but also for the increased sociality and creativity during this phase of life. According to Crone, teenagers have difficulties understanding emotions and recognizing facial expressions of others, and they also struggle to take the perspectives of others into account. Because the frontal lobe is not yet able to regulate the heightened emotionality of adolescence, teenagers are said to be more sensitive to social exclusion than adults (Crone, 2012, p. 91). Similarly, the presence of peers gives teenagers the pleasant feeling of a reward, which explains their increased risk-taking behaviour when peers are present. Finally, Crone explains how the PFC thesis makes teenagers many times more creative, idealistic, and inventive than adults. During adolescence, brain areas that are deemed important for creativity, resourcefulness, musicality, sports, and social involvement are the last to be subjected to the pruning process (Crone, 2008, p. 
150). The lack of performance of the prefrontal cortex becomes beneficial, as it might be hinder the formation of creative ideas or the performance of behaviours such as sincerity, political engagement, and idealism, all of which Crone considers to be typical of the adolescent brain.

\section{Making the Teenage Brain Valuable for Parents and Pedagogues}

Since the publication of her books, Crone has been readily seen as the Dutch expert on adolescent behaviour and is frequently featured in national newspapers, TV shows, reports, and magazines. ${ }^{25}$ In her books and numerous interviews, Crone appears conscious of the possible relevance of her research as well as the high expectations parents, policy-makers, and others could have about her research subject. She carefully balances these expectations when translating her research to a wider audience. As described in the previous chapter, I use the term value work to describe and call attention to the deliberate efforts of attributing relevance and meaning to scientific claims by enacting, articulating, and contesting values. As such, value work is often directed to a particular audience - in this case, parents and pedagogues. We can discern several strategies of value work employed by Crone: addressing parental anxieties and articulating parental concerns; spelling out parenting norms while carefully coordinating the normative uptake of her work; and anticipating conflicts by combining claims of novelty and epistemic certainty with stressing historical continuity and acknowledging limitations of her research.

In her popular books, Crone aims to speak directly to parents by addressing parental anxieties and articulating parental concerns regarding their teenagers. She appeals to parents' insecurities by describing adolescence as confusing, unpredictable, impossible, and a mystery (Crone, 2008, p. 8-9) - implying that predictability and being knowledgeable are important for parents. Crone gives everyday parenting examples of topical concerns such as the teenage wish for driving a moped before the age of sixteen, buying clothes, unrealistic mood swings, dangerous games (such as the choking game), alcohol use, CITO-test performance (Dutch high school test scores), and problems completing homework. These concerns are then related to the pace and development of specific parts of adolescent brains (the PFC thesis), turning the teenage brain into a diagnosis for these problems. Parents are encouraged to make an effort to under-

${ }^{25}$ Crone received many grants and awards for her work on adolescent brain development. Just recently (June 16, 2017), she received the Spinoza Prize, the highest Dutch scientific award, for her "pioneering" and "internationally ground-breaking research" (NWO, 2017). 
stand the complexities of their teenager's brain in these examples, implying that an increased knowledge and understanding would be reassuring and would lead to empathy, (generational) solidarity, and a better judgement. Parents are cast as being in need for such knowledge, which is promised to empower them in their daily dealings with their adolescents.

How parents precisely should use this knowledge remains vague and ambiguous. As a second strategy of value work, which can be observed both in her books and in interviews, Crone tries to coordinate the uptake of her work by balancing between maintaining a firm boundary between science and parenting/pedagogy and showing the value of her work for parents by spelling out some parenting norms. For example, she mentions in the introduction of Het Puberende Brein (2008): "The book is not meant as a guidebook for how, as a parent, to deal with your teenager, rather it is meant to gain more insight in teenage brains and the changes that take place in them" (Crone, 2008, p. 9). And in the second book: "This (again) is not a book about parenting . . . I am not a pedagogue" (Crone, 2012, p. 179/180). When asked whether she gives advice, Crone states:

I don't do advice. I do get questions, for example when someone's teenager did something stupid. But I will never say to parents how they should solve it. At most, I'll explain why it's good when teenagers experiment and acquire new experiences. Just my well-known claim. (Crone, in Goslinga \& Spiering, 2012, p. 16)

While Crone is hesitant and careful not to promise too much, she struggles to maintain a clear boundary between her scientific research and the potential relevance of her work for parents, pedagogues, and others. She repeatedly gives explicit and normative parenting suggestions despite her claims to the contrary. For example, interviews she has said things like: "To just let go is not right. But being very strict and authoritative, to be on top of them, is also not right" ("Hoe werkt een puber?" 2012, p. 6), "the model of management by negotiation is good because you can then explain your reasons" (Crone, 2007), or "I plead for mildness with rules. The biggest challenge for parents in puberty is to let go" (Hamer, 2012, p. 9). She often mentions she "takes sides with the teenager" (Goslinga \& Spiering, 2012, p. 16) against worrisome and meddlesome parents, and that she aims to "create an understanding amongst parents and teachers" ("Hoe werkt," 2012, p. 6), since teenagers have a negative image. This way, Crone aims to add an emancipatory relevance to her work (for adolescents) by 
explaining "where their behaviour comes from" ("Hoe werkt," 2012, p. 6) and implicitly promising that this knowledge comes with a 'right' course of action for parents.

As justification for the promise of applicability, Crone presents neuroscience knowledge on adolescent brain development as a "completely new perspective on the behaviours and motivations of adolescents," with "spectacular insights" on adolescence, which is cast as "one of the least well-known periods in child development" (Crone, 2008, p. 8, 11). A new insight that Crone often puts forward is her claim that virtues and ideals such as creativity, sociality, idealism, and sincerity are typical for adolescent brains, by which she contests negative stereotypes of teenage behaviour. At the same time, Crone puts her work into the historical tradition of Hall's 'Storm and Stress,' and other scholars in the field of developmental psychology, such as Piaget, Kohlberg, and Westenberg (Crone, 2008, p. 15).

After her second book, Crone appears more cautious to claims of epistemic certainty. For example, Crone mentions in an interview that:

We must not exaggerate the influence of brain science. What do we know at this moment? We know a few patterns of brain activity that correlate with particular behaviours. An island in an enormous sea of brain activity, of which we mostly understand nothing. (Crone, in Goslinga \& Spiering, 2012, p. 16)

This is a third strategy of value work: balancing between novelty and tradition, between epistemic certainty and acknowledging limitations of brain research. This strategy not only is geared at establishing scientific credibility but also is aimed at anticipating possible conflicts and critique. After Crone, together with international renowned paediatrician Ronald E. Dahl, published a metaanalysis in Nature Reviews Neuroscience in 2012, the epistemic certainty and normative implications of her work became subject to some debate in the Dutch public sphere. In the Nature article, Crone and Dahl claimed that the maturing prefrontal cortex-thesis is too simplistic, and that "developmental neuroimaging studies do not support a simple model of frontal cortical immaturity" (Crone \& Dahl, 2012, p. 636). Crone was vehemently criticized by developmental psychologist Willem Koops (amongst others) for offering parents a "false certainty" that had let them believe that "they can't or shouldn't do anything" since the teenage brain was to be seen as the cause of adolescent problematic behavior (Koops, 2013, p. 19). Crone states that parents, teachers, and psycholo- 
gists nonetheless have "a right to know what we managed to determine," even if "not all results bring 100\% clarity" (Crone, 2013, p. 17-18). Crone thus deflects the critique by referring to her explicit acknowledgement of uncertainties in her popular-science books, stating that this uncertainty does not dismiss the potential positive relevance of her work.

Crone thus faces the same struggle in which many neuroscientists find themselves: manoeuvring between the high expectations of parents, pedagogues, and journalists, and making a highly technical and relatively young field of research relevant for a wider audience. As we have seen, such manoeuvring calls for strategic value work. At times, Crone reflects on her ambivalent attitude:

It is complicated. You do want that people do something with your book. On the other hand, I find it difficult when I notice that its contents are not being applied in the right way. (Crone, in Van der Heijden, 2013)

What the right way is according to Crone remains unclear. While she addresses several parental concerns and gives a clear diagnosis of teenage behaviour (the PFC thesis), her suggestions to parents about what to do (action programmes) and for what reasons (ideals) are ambiguous. Through her value work, Crone reaffirms and constructs herself as a trustworthy scientist who seems only interested in the facts, while at the same time giving mixed messages to parents, pedagogues, and others about the potential relevance and value of the teenage brain for parenting and what they could and should do with their teenager's brain. More importantly, by articulating parental anxieties and concerns, spelling out some parenting norms, and anticipating conflicts and critique, Crone prepares the ground for parents', pedagogues', and others' moral argumentation about adolescent behaviours and brains and for their normative appropriation of her work. As we will see next, this, in turn, opens the door to multiple, sometimes conflicting moral repertoires about what parents could and should do with their teenager's brains.

\section{The Teenage Brain as Parenting Advice}

The PFC thesis quickly found its way into Dutch public discourse. After widespread dissemination through all kinds of media, the notion of the teenage brain became commonplace when addressing adolescents, a part of the symbol- 
ic toolkit used to explain adolescent behaviour and parenting issues. Between 2008 and 2015, parenting adolescents became a topic frequently discussed in public debate. This is partly due to the activities of the specially appointed Minister of Youth and Family under the Christian and social democratic government of Balkenende IV (who organized activities such as a nationwide online Parenting Debate in 2010). But this spike in interest was also partly due to the 'Facebook-riots': a birthday party that spiraled out of control, resulting in riots in the city of Haren and a fierce public debate about the attitude of adolescents and parents and the role of social media. ${ }^{26}$ The teenage brain became a popular explanans to account for topical issues such as street violence, the radicalization of young Muslims travelling to Syria, and other excesses such as youth suicides due to bullying. Crone's work found especially willing audience in pedagogues, family coaches, (self-declared) parenting experts, and parents, all of whom eagerly adopted the concept of the teenage brain to discuss parenting issues.

After having described Crone's efforts to make her research valuable to parents and pedagogues, I now trace valuations of Crone's teenage brain in parenting books and parenting magazines. I synthesize the mobilization of valuations in concerns, promises, and expectations by pedagogues and parenting experts in terms of therapeutic repertoires: recurring configurations of a parental challenge or parental concern, a diagnosis of adolescent behaviour in terms of the brain, a parental action programme (what parents can or should do), and an ideal of good parenting to strive for. Therapeutic repertoires are analytically distinct; in discursive practices, they are often used together, sometimes even in the same newspaper or magazine article. By explicating these repertoires, I will show how precisely ideas of parenting well with a teenage brain are constructed in practice. ${ }^{27}$

Pedagogues and parenting experts share Crone's promise that knowledge of adolescent brain development results in a new and better understanding of teenage behaviour that can help parents deal with their children during adolescence. Following the maturing prefrontal cortex (PFC) thesis, parenting experts take up Crone's claim that parents should be the external frontal lobe of their

\footnotetext{
${ }^{26}$ An appointed investigation committee mentioned the teenage brain as an underlying cause of the YOLO attitude of youngsters during the riots (You Only Live Once, a modern equivalent of carpe diem): "processes that, according to scientists, relate to the uneven development and defective integration of the young brain, played a prominent role during the riots in Haren" (Van den Brink et. al., 2012, p. 22).

${ }^{27}$ To be clear: self-knowledge (one of the elements of a good life) here relates to the knowledge parents (should) have about their adolescent children.
} 
teenagers (Crone, 2012, p. 181; Houtekamer, 2010, p. 6). Since the prefrontal cortex of teenagers is still in development, parents are urged to complement or take over its functions, especially cognitive functions such as planning, overseeing long term consequences, and risk assessment. However, valuations diverge in how to be a good frontal lobe, what parents should do, and for what goal or ideal, resulting in the emergence of two distinct therapeutic repertoires of parenting a teenage brain. In the first repertoire, parents are cast as akin to guardians of external stimuli, whereas in the second repertoire, parents are seen as supportive coaches. The teenage brain gains traction precisely because it is made valuable for different ideas of good parenting and combines them, while at the same time reconfiguring them in terms of the brain.

\section{Therapeutic repertoire I: Parents as protective guardians of external stimuli}

To the path of a matured brain, lots of risks loom around the corner. The teenage brain is often used as an explanans for indicating why certain prevalent adolescent behaviours - often seen as increasing in number - are harmful for teenagers and their brains. This repertoire is especially present in popular mass media, where publicly reported topical excesses such as street violence or binge drinking result in cries by commentators and experts for parents to take more responsibility and "finally start parenting again" (Den Bljker, 2012, p. 8). For parents, the challenge in this repertoire is how to deal with those risky behaviours - especially those that potentially physically hamper adolescents' natural brain development. The most frequently discussed danger for adolescent brains is addictive behaviour, whether related to the use of alcohol, tobacco, or other drugs (e.g., cannabis, speed, or gamma-Hydroxybutyric acid, better known as the party drug GHB) or to gambling, excessive eating, or any of the 'new' addictive risks such as gaming and social media use. Alcohol and drugs remain the most dangerous risks for a developing teenage brain, and as such are strongly condemned. Another perceived and topical danger for the teenage brain is digital technology. Parents seem to struggle with smartphones and internet use in their households. Problems such as cyber bullying, excessive gaming, internet porn addiction, and heavy smartphone use are frequently featured topics in parenting magazines and books.

The heightened emotionality and impulsiveness of the teenage brain are used in this repertoire as diagnoses - ways to account for and warn against irresponsible risk-taking behaviour. We can read an example of this diagnosis in the popular Dutch parenting magazine $J / M$ : 
A teenager is inclined to take decisions that result in a short-term reward. Because of this, he quickly decides to do irresponsible things: eating too much, too fat or too sweet, practicing dangerous sports, like the newest fad to jump off of buildings or from roof to roof. As long as it gives an immediate kick. Teenagers do not see risks at that moment. (Eerkens, 2007, p. 24)

Because of the PFC thesis, teenagers are said to be prone to obsessive and monomaniac behaviour (impulsivity combined with a focus on short-term rewards); therefore parents are urged in this repertoire to set clear rules for computer, internet, and phone use to prevent addictive and obsessive behaviour. Knowledge about the teenage brain is used here as an unquestionable biological justification which urges parents to protect their children's development during adolescence.

Parenting-as-external-frontal-lobe is concerned here with protection from risk. The disruption of the 'natural' growth of the developing brain of teenagers is seen as the greatest danger, and fear of such a disruption occurring is used to urge parents to be more directive and protective. Intended to protect the natural development of adolescents' brains, action programmes for parents consist of setting and upholding rules by using strategies of discipline and lowered expectations. Two main strategies of disciplining are proposed in this repertoire: prohibition and external motivation through reward or punishment. In the case of alcohol for example, postponing its use should be achieved by strictly prohibiting it without discussion, as alcohol and drugs are seen as too dangerous for the teenage brain to leave it as a matter of negotiation. Less dangerous risks such as internet and smartphone use should be limited by setting rules through agreements with teenagers, consistently upholding these rules, and providing rewards when the rules are followed. Stressing the emotional sensibility of teenagers and giving direct rewards is preferable to punishment because, as Crone pointed out in a 2010 interview, "young brains learn the most" from rewards (Korteweg, 2010, para. 4). When upholding certain rules or limits, parents should "dare to seek confrontations, not avoid difficult conversations, and demand obedience" (Jansen Schoonhoven, 2013, p. 21). Or, as popular selfdeclared teenage experts Marina van der Wal and Jan Dijkgraaf ironically phrase it: "Treat them like dogs: reward good behaviour. . . . Just like you'll catch flies with syrup, you'll catch teenage attention and good will with compliments" (Van der Wal \& Dijkgraaf, 2013, p. 55). 'Bad' parents in this repertoire 
are those who want to be friends with their children instead of exerting a certain amount of control through discipline or prohibition.

Pedagogues often mobilize the example of tiger parents - parents who have high expectations of their adolescent - as a bad example. Parents in this repertoire instead should lower their expectations, as adolescents are not able to fully use their frontal cortex. For example, Geraldien Blokland, pedagogue and coach at the Dutch Youth Institute, argues in popular parenting magazine J/M:

For a long time, our expectations of teenagers were too high. We wanted to take them seriously, because they look so mature and because they themselves want to be taken seriously. But the fact is that you cannot expect them to be all that reasonable. (Blokland, as cited in Horsthuis, 2008, p. 13)

As a consequence, parents should be more proactive and "help teenagers with everything their brains have difficulties with" (Pardoen, 2008, p. 10). The maturing PFC thesis is used here to frame teenagers as a kind of defective adults: their brains are 'work in progress', not there yet, and their brains are just "wrong" compared to the right brains of adults ("Foute afstemming puberhersenen," 2008, p. 5). The susceptibility of the plastic teenage brain is valued as a vulnerability, by which the focus shifts to risks that might harm or disrupt its natural development. The developmental perspective of natural brain development in adolescents is valued here as needing a particular kind of guidance, correction, or protection. As a result parents, acting as substitute brains, become akin to guardians that shield the teenage brain from harmful stimuli present in the outside world and are responsible for the natural development of their adolescents' brains.

The teenage brain is heralded by parents and pedagogues in this repertoire as justification for the idea that teenagers 'really' are different; It thus allows them to construct and internalize the idea that their children are not abnormal. By rooting teenage behaviour in the brain, the differences between adults and adolescents become normalized; impulsivity and riskiness should be expected, even accepted, as being normal for this stage in life. Why is normality valued so highly by parenting experts and pedagogues in this repertoire? In what kind of environment or society does normality emerge as an ideal? Apart from the concerns already mentioned - addictive behaviours to (new) drugs, smartphones, and social media - there is a not-so-silent elephant in the room in this repertoire. The environment in which the role of being a good parent is to be played 
in this repertoire consists of a society in which abnormal behaviour is on the rise and increasingly medicalized.

Magazines and parenting books often contain advice on how to recognize abnormal teenage behaviours that could be symptoms for diagnoses such as ADHD, autism, depression, and dyslexia. As a study on Dutch parents found, a majority of parents are concerned by the medicalization of children's behaviour, especially the rise of ADHD, autism, depression, and dyslexia, which are said to have their onset in adolescence (Brinkgreve, 2012). In the past decade, parents have become accustomed to diagnoses and labels of mental disorders that introduce neurobiological knowledge into their (parenting) lives. As a consequence, the value parents place on normality seems to increase. In such a context, where emotional outbursts, impulsive choices, monomaniac use of smartphones, or lack of attention for homework can be perceived as potential symptoms of a mental disorder, it can come as a reassurance and relief to parents to hear that these behaviours are not abnormal but part of a normal process of brain development. The teenage brain in this repertoire thus seems to respond to the trend of the medicalization of abnormal behaviour by providing a diagnosis in a similar biological language (neurobiological knowledge) without turning adolescents into potential patients. What's more, in contrast to the emergence of mental disorders such as ADHD or dyslexia, it gives parents an active role in securing an undisturbed, normal development of their adolescent's brains. While (societal) developments in the environment are cast as beyond parental control - parents cannot influence medicalization of behaviour, or the rise of smartphones - they are given the capability to protect their adolescent's brain development by shielding them from harmful external stimuli.

To summarize this therapeutic repertoire of being a good external frontal lobe: The diagnosis of teenage behaviour is described here as heightened emotionality, impulsivity, and riskiness - teenagers are cast as defective adults. Parents should therefore be directive and protective of their adolescent's brains using disciplinary techniques and by lowering their expectations. The ideal parenting outcome in this repertoire, what adolescents could become, is formulated in terms of a normal or natural brain development. The environment in which this ideal is deemed important is a society in which (new) addictive behaviours are on the rise and where parents have become accustomed to the medicalization of abnormal behaviours. Parents are thus encouraged by pedagogues and parenting experts to actively protect their adolescent from harmful external stimuli. This amounts to the model of good parenting shown in Figure 3. 


\begin{tabular}{|c|c|c|c|}
\hline \multicolumn{4}{|c|}{ Therapeutic repertoire I: Parents as guardians of external stimuli } \\
\hline & \multicolumn{2}{|l|}{ SELF-KNOWLEDGE } & \multirow{2}{*}{$\begin{array}{l}\text { PRACTICAL OPTIONS } \\
\text { Action programme }\end{array}$} \\
\hline & Diagnosis & Ideals & \\
\hline SELF & $\begin{array}{l}\text { Teenager as defective adult } \\
\text { with vulnerable plastic brains } \\
\text { (emotionality, impulsivity) }\end{array}$ & $\begin{array}{l}\text { Normal, undisturbed } \\
\text { brain development }\end{array}$ & $\begin{array}{l}\text { Prohibition, punishment, } \\
\text { praise (disciplining) }\end{array}$ \\
\hline ENVIRONMENT & \multicolumn{2}{|c|}{$\begin{array}{l}\text { Rise of harmful stimuli and medicalization of } \\
\text { abnormal behaviour }\end{array}$} & $\begin{array}{l}\text { Influence of } \\
\text { environment can be } \\
\text { controlled }\end{array}$ \\
\hline
\end{tabular}

Figure 3. Therapeutic Repertoire I: Parents as guardians of external stimuli. In this repertoire, parents work to protect the normal brain development of their adolescent through prohibition, punishment, and reward.

\section{Therapeutic repertoire II: Parents as supportive coaches}

In the second therapeutic repertoire, the teenage brain is made valuable for addressing a different challenge for parents: how to get the best out of their teenager. Being an external frontal lobe in this repertoire means that parents should give their teenagers some freedom to experiment, as the teenager is "enterprising, is open for many stimuli, focused on challenges, and thus needs to gain experiences," as well-known neuropsychologist Jelle Jolles summarises (Jolles, 2011, p. 16). The teenage brain is used here to talk about the role of parents in the process of adolescent self-actualization. The underlying developmental perspective is that of a developing adolescent brain that seeks new experiences and new input to mature. To reach the goal of a mature brain in adulthood, a natural development of adolescent brains includes exploring, experimenting, and acquiring different experiences.

For example, regarding social exploration and experimenting with social identities, it is often suggested in parental magazines that parents give their adolescents some slack and offer them the opportunity to acquire new experiences for themselves. As Justine Pardoen, founder of Ouders Online (a popular online parenting community in the Netherlands) suggests:

Give a teenager the chance to do things which you as an adult don't like (anymore), such as watching horror movies, big physical challenges, or other exciting stuff. A teenage brain not only fancies excitement, but by doing so it has an opportunity to learn to deal with problems and feelings of fear. (Pardoen, 2008, p. 10)

Similarly, parents should not protect or prevent their adolescent from acquiring negative or disappointing experiences. Regarding cognitive tasks, such as 
homework or planning, parents should help and support where possible - but not take over. Teenagers themselves must learn to plan their homework, helping them doing their homework is out of the question. Parents who interfere too much with their adolescents, often labelled helicopter parents, are strongly condemned.

The plasticity of teenage brains is valued here as an opportunity; adolescence is thus seen a unique phase in life in which the brain is able to grow and develop the most. Consequently, teenagers with their plastic brains are each framed as unique individuals: "Teenage brains are not like unfinished adult brains; they are creative innovation and learning machines, with their emotional circuits focused on new experiences and exploring social contacts," as science journalist Malou van Hintum paraphrases Crone (Van Hintum, 2012, p. 6). Parents in this repertoire are encouraged to give teenagers some room to develop their brains. Parents still need to set some rules and limits, but not for reasons of protection. Rather, such rules and limits are seen as being necessary for the process of self-actualization. The idea is that teenagers need to have some limits or rules against which they can rebel. So when setting rules and upholding them, parents should refrain from punishments or rewards, but focus rather on intrinsic motivation, negotiation, mutual agreements, and making decisions together. Even regarding alcohol, which led experts in the first repertoire to stress the need for strict prohibition, experts in this repertoire cling on to the need to let adolescents themselves make the right choices, as we read in this workshop about Crone's teenage brain at a high school in Amsterdam:

Reward or punishment for good or bad behaviour doesn't work anymore. Rather, what's important is to think along with the teenager, discuss different options together, clarify consequences and discuss what the (logical) effects could be of their actions - for example regarding traffic, alcohol and drug use. Try to stimulate development by letting them make choices from different options. That's how a teenager learns to make a rational judgment, and it brings ratio and emotion faster in balance. They still have to learn the chain of cause and effect. Dedicated, non-patronizing support of parents can help them with this. (Dugomay \& Mok, 2009, p. 10)

In contrast with the first repertoire, parents should not draw the conclusion that teenagers are not ready for adult behaviour because their brains aren't finished. 'Bad' parents in this repertoire are those whose expectations of their adolescents 
are too low and thus don't take the needs of the adolescent (brain) seriously enough (described as neglectful or permissive parenting styles). Instead of low expectations, parents in this repertoire are encouraged to actually raise their expectations. As developmental psychologist Steven Pont, a well-known critic of Crone, argues:

The right conclusion should be: precisely because the brains of teenagers are not mature yet, we have to stimulate and challenge them. Adult requirements make you mature, even if you're not. Just like you're not waiting to teach your child how to talk, until the moment that he actually can already. (Pont, 2010, p. 3)

Instead of focusing on behaviours such as irrationality or riskiness, the focus in this repertoire shifts towards more positively evaluated behaviours as characteristic for the teenage brain: behaviours that stress opportunities to excel. The maturing prefrontal cortex thesis is valued here with an emphasis on the increased sociality and creativity of the teenage brain. Teenage behaviours that are deemed as unequivocally beneficial for their developing brains are behaviours that stress creativity and out-of-the-box thinking, sociality, (political) idealism, and sincerity. These character traits, framed as typical of a teenage brain, are seen as opportunities that should be cherished and encouraged by parents because they allow their adolescents to act as unique individuals. Parents in this repertoire thus resemble supportive coaches, only curbing the freedom of their adolescents in ways that will help them flourish and excel.

The intrinsic qualities of a teenage brain, as celebrated by Crone along with the parenting experts and pedagogues in this repertoire, are virtues such as creativity, flexibility, and sociality - not coincidentally, virtues that we deem important in our post-industrial, meritocratic knowledge economy. Why do pedagogues and parenting experts hammer on these virtues? What kind of environment requires teenagers to be creative and flexible? Contemporary Dutch parents seem anxious and concerned about raising adults-to-be in our contemporary society; one out of two parents indicate that they feel responsible for the happiness and societal success of their children, as a study on ideas of Dutch parents by sociologist Christien Brinkgreve indicates (Brinkgreve, 2012). The teenage brain appeals to these parental anxieties and ambitions by promising parents all the good things while taking away the fear of becoming a 'hyper parent' or 'helicopter parent.' It assures parents that the character traits for societal success - creativity, flexibility, sociality - are already dormant in the ado- 
lescent brain. Normality here does not refer to an undisturbed natural development but instead means adhering to the norms of success in our contemporary society. While parents are encouraged to stimulate these character traits, they are mostly warned not to interfere too much so that these character traits for success can blossom - a message that releases them from the dreaded fate of becoming 'control freaks' (helicopter parents). While societal success is presented as beyond the scope of parenting, the teenage brain in this repertoire, summarized in Figure 4, reassures parents that this is not problematic, as the teenage brain already possesses the virtues it needs to be successful. All that's left for parents to do is to stimulate their adolescents in the right direction.

\begin{tabular}{|c|c|c|c|}
\hline \multicolumn{4}{|c|}{ Therapeutic repertoire II: Parents as supportive coaches } \\
\hline & \multicolumn{2}{|l|}{ SELF-KNOWLEDGE } & \multirow{2}{*}{$\frac{\text { PRACTICAL OPTIONS }}{\text { Action programme }}$} \\
\hline & Diagnosis & Ideals & \\
\hline SELF & $\begin{array}{l}\text { Teenager as unique individual } \\
\text { (creativity, sociality, etc.) }\end{array}$ & $\begin{array}{l}\text { Teenage (brain) } \\
\text { self-actualization }\end{array}$ & $\begin{array}{l}\text { Stimulation, support, } \\
\text { steering (motivating) }\end{array}$ \\
\hline ENVIRONMENT & \multicolumn{2}{|c|}{ Complex, meritocratic knowledge society } & $\begin{array}{l}\text { Societal success beyond } \\
\text { control of parents }\end{array}$ \\
\hline
\end{tabular}

Figure 4. Therapeutic repertoire II: Parents as supportive coaches. In this repertoire, parents are urged to encourage the self-actualization of their adolescents, by motivating teenagers to develop their unique character traits.

\section{Taming the Future: moral changes in parenting practices}

These two therapeutic repertoires make visible how the teenage brain is made valuable in Dutch parenting discourse in ways that propose different and sometimes conflicting parental norms and ideals. The teenage brain appears to be versatile enough to reconcile different epistemic and normative assumptions regarding teenagers and parenting, strengthening moral positions on protective parenting and adolescent self-development. The distinction between the two therapeutic repertoires is analytical; in practice, experts in parenting books and magazines easily switch between the two therapeutic repertoires and suggest parents both be more strict and let go at the same time. The message is thus that acting as an external frontal lobe entails balancing between protecting teenage brains from dangerous stimuli (control) and stimulating teenage brains for selfactualization (freedom). 
The promise that Crone, pedagogues, and parenting experts share is that knowledge of adolescent brain developments brings a new understanding of adolescent behaviour which would aid parents to decide what course of action to take when dealing with their adolescent's behaviour. After explicating how and for what reasons the teenage brain is used as parenting advice, we are now able to scrutinize this promise of neuro-moral change. To what extent does the teenage brain lead to a changed understanding of adolescents? And to what extent does the emergence of these two therapeutic repertoires about what parents could and should do with their adolescent's brains actually challenge parenting norms and ideals? As we will see, the teenage brain as parenting advice does not result in revolutionary new understandings of what it means to be an adolescent, or what parents should do to parent well. However, the two repertoires on parenting the teenage brain do indicate some shifts between views of adolescence and parenting ideals.

\section{The Neurological Adolescent: the normalization of differences}

To what extent does knowledge of adolescent brain development shed new light on the phenomenon of adolescence? In her two popular science books, Crone focusses on specific forms of adolescent behaviour, notably the relation between cognition and emotion that results in increased risk-taking behaviours, sociality, and creativity. Crone's choice to emphasize the brain activity of these particular behaviours reflects the way adolescents are imagined throughout history. The struggle between reason and the passions, as observed by Aristotle and continued by Hall's 'Storm and Stress' and 'normative turmoil,' are now redescribed in neurological terms through the maturing prefrontal cortex thesis: as a difference in the pace of development between the prefrontal cortex and the nucleus accumbens. Where a lack of adolescent discipline in Victorian times was attributed to a lack of character, now the lack of adolescent self-control is explained by referring to their immature prefrontal cortex, the area of the brain that is seen as the seat of self-regulation and self-management.

The teenage brain as a model of growth and development becomes described in the two repertoires in terms such as work in progress, immature, unfinished, undeveloped, malleable, in transition, in motion, flexible, dynamic, unbalanced, imperfect, sensitive, and vulnerable. Metaphors used are the building of small roads and highways, the metamorphosis of a larva, a learning machine, and a natural ripening. Where Hall (1904) spoke of adolescents as 'becoming,' the phrase now has its neuroscience equivalent in the plasticity of the 
teenage brain. ${ }^{28}$ Knowledge of adolescent brain development thus does not qualify as revolutionary or spectacular; it doesn't challenge our existing understanding of adolescence as a period of normative turmoil but rather re-describes it in neurological terms.

The plasticity of the teenage brain in this case is described in developmental terms - as the forming of the structure and functions of the brain during a sensitive period of growth. The teenage brain is thereby presented as particularly impressible to environmental stimuli: In this view of plasticity, agency is given to the environment, which is said to shape the teenage brain. The two therapeutic repertoires diverge in their assessment and valuation of the susceptibility of the teenage brain. In the first repertoire, the 'openness' of the teenage brain is seen as an undesirable vulnerability, as external stimuli could potentially harm its 'normal' development. In the second repertoire, the susceptibility of the teenage brain is valued as a desirable opportunity: It enables teenagers to learn and to acquire much needed experiences that shape their brains for the better. By seamlessly slipping from one plasticity into the other, parental suggestions based on protection from risk (repertoire 1) and on stimulation of experiences (repertoire 2) can be combined.

This re-description of adolescent behaviours in terms of their plastic brains has some effects. First of all, rooting behaviour in the brain effectively normalizes teenage behaviour. The maturing prefrontal cortex thesis is used in both therapeutic repertoires to frame adolescents as a distinct category of people with shared biological characteristics. This 'neuroessentialist' view is based on the assumption that there is a single brain type that would be common for all members of this category, regardless of individual differences or other differences such as sex, gender, or social-economic class ( $\mathrm{O}^{\prime}$ Connor, Rees, \& Joffe, 2012). ${ }^{29}$ Referring to their distinctive brains, teenagers become cast as all that

\footnotetext{
${ }^{28}$ The idea that teenage brains are 'not finished yet,' presented as revolutionary and necessary knowledge for parents, can actually already be found in Hall's work as Arnett (2006) notices: "This causes increased thickness of the parietal and central regions, some in the temporal, and is probably followed by growth in the frontal regions last, all this occurring in regions that were far less rich in fibers in early adolescence. The development of these fibers seems to follow more closely than any other brain structure we know the growth of intelligence. . . . I think we may say that, at last, we seem to have within our reach some conception of what brain changes are involved in adolescence." Hall (1904) (as cited in Arnett, 2009)

${ }^{29}$ Strikingly, while Crone does elaborate on it in her books, in the discourse on parenting teenage brains, differences between boys/girls, or stages in adolescent development, are only occasionally made. In contrast to the context of education, where sex/gender differences between boys' and girls' brains are a topic of fierce discussion, the teenage brain in the context of parenting is presented
} 
adults either should not want to be, or can't be: Teenagers are impulsive, irrational, irresponsible, and impulsive, but they are also creative, idealistic, and flexible - implying that, as their opposites, adults are controlled, rational, responsible, balanced, uncreative, practical, and inflexible. As a consequence, adolescents become symbolically distanced from adults. This distance leads those around them to believe that they also should not be treated as adults, which in turn increases the need for parents to assert themselves more.

In recent years, some scholars have argued against the normalization of the neurological adolescent. For example, Choudhury (2010) argues that the 'turbulent' teenage brain is often used as a means for explaining limitless behaviour and 'youth gone wild,' thereby reiterating stereotypical images and prejudices about adolescents (Choudhury, 2010). Others question the impulsivity and risktaking assumptions in neuroscience research (e.g., Bessant, 2008; Males, 2009), or scrutinize whether an immature PFC would result in a diminished responsibility and culpability in the context of legal decision-making (e.g., Luciana, 2010; Ryberg, 2014). These authors argue against a re-iteration of negative stereotypes of adolescents as risky and impulsive, as it would result in further (social) control of their behaviour and diminished responsibility. ${ }^{30}$ While the case of Crone's teenage brain in the context of parenting confirms this redescription of negative adolescent stereotypes in terms of the brain, especially in the first repertoire, it also shows something that contrasts these studies.

In the case of parenting, the normalization of teenage behaviour entails not only negative stereotypes but also positively valued ones. Just as Hall cherished the vitality of adolescents, Crone celebrates and emphasizes their sociality, (political) idealism, flexibility, and creativity - virtues we deem important in our contemporary knowledge society. Crone values the undesirable stereotypical aspects - or, to speak with Hall, the 'primitiveness' - of adolescent behaviour, such as risk-taking, recklessness or unthoughtful behaviour, as problematic, especially for parents: They have to learn to understand and adjust. Crone does not condemn or blame teenagers for exhibiting these behaviours (especially their sociality, emotionality, and lack of rationality); they are presented as part of a normal brain development which "will pass by itself" (Houtekamer, 2010,

mostly as a homogenous entity. The same goes for parents: There is almost no differentiation in parenting discourse between fathers and mothers.

30 Since April 1, 2014, with the introduction of adolescent criminal law, adolescents form a distinct group in the Dutch law system. This law was proposed by minister Fred Teeven by referring to the developing teenage brain, especially its risk-taking behaviour due to a perceived lack of ability to foresee long-term consequences (Teeven, 2012; RSJ, 2011). 
p. 6). The aim of Crone to elevate the negative stigma of adolescents by emphasizing their unique character traits is succeeded by parenting experts and pedagogues, as the emergence of the second parenting repertoire shows. While a reading of adolescence as having a kind of vitality already started with Hall, this emancipatory view has now gained weight and visibility, as it is often used to debunk and contest the dominant view of the problematic teen.

This emancipatory effect of rooting teenage behaviour in the brain echoes arguments used in the neurodiversity movement. In this civil rights movement, born out of the autism rights movement, neurobiological differences among people are seen as natural variations instead of pathologies or defects. The claim is that neurodiverse individuals, or 'non-neurotypicals,' as some of these proponents (such as people with autism) call themselves, should therefore not be stigmatized or paternalized but rather be accepted (Jaarsma \& Welin, 2012; Ortega, 2009). In this case, too, knowledge of adolescent brain development is used to argue for more acceptance and tolerance amongst parents, especially regarding the different, negative behaviours of their adolescents (the first repertoire).

In a more radical version of neurodiversity, neurobiological differences should not only be seen as biological justifications for normalizing and accepting differences between neurotypicals and non-neurotypicals but should even be celebrated as unique or superior to neurotypicals (i.e., 'normal,' able people without disabilities or disorders). We have seen similar arguments in the second repertoire, where adolescents are framed as much more creative, flexible, and social than adults - character traits that should be stimulated and cherished by parents. The result of this naturalization of both undesirable and desirable stereotypes of adolescents is that parents and pedagogues not only should accept the differences between them and their adolescent children as being normal but also should expect their adolescents to be different - to be risky, impulsive, creative, and idealistic - to display the behaviours that now count as normal for teenagers.

\section{The Teenage Brain as a Balancing Tool}

The promise of many pedagogues is that knowledge of teenage brain development helps parents in dealing with their adolescents. So to what extent does knowledge of the teenage brain challenge the practical options parents have for parenting their adolescent children? An important consequence of normalizing teenage behaviour through knowledge of their plastic brains is that the focus 
shifts to their parents; It's they who have to be raised by learning how to take the brain development of their adolescents into account. The principle imperative of the teenage brain for parents is thus that if parents (and others) want to understand teenagers and deal with their behaviour, they have to start by understanding adolescent brain development - experiential or anecdotal advice from other parents about how to raise adolescents is not enough. As such, the teenage brain as parenting advice is no different from other science-based parenting advice: The virtue of (scientific) knowledgeability has been presented as a prerequisite for being a good parent since the rise of parenting experts (cf. Wubs, 2004; Wesseling, 2002).

As we have seen in the two repertoires, the teenage brain was used to mobilize different ways of parenting: from prohibition, punishment, and praise (disciplining), to stimulation, steering, and support (motivating). Similarly, parents were told to either have low expectations since their teenagers are 'defective adults,' or to have high expectations as their adolescents are 'unique learning machines.' These styles of parenting are obviously not new. According to Depaepe et al. (2005), every form of pedagogisation, both past and contemporary, is characterized by an inherent paradox: pedagogy is always about both enabling freedom and exerting control, about emancipation and paternalism, about coercion by the parent and the liberating experience of the learning individual (Depaepe, 2005, p. 35). The teenage brain as parenting advice does not escape this paradox.

The appeal of the teenage brain as parenting advice does not seem to lie in providing new practical options, but rather in combining (at times) conflicting ways of parenting. According to many contemporary Dutch pedagogues and sociologists, as well as many parents, the contemporary Dutch parenting attitude can be characterized as an authoritative parenting style (Baumrind 1991; Brinkgreve, 2012; De Winter, 2005; Maccoby \& Martin, 1983; Pronk, 2013). The authoritative parenting style (not to be confused with an authoritarian parenting style) can be described as 'a balancing act,' or a golden mean, between selfactualization (of the adolescent) and protection from dangers. After a focus on equality and mutual agreement that characterized parenting styles in the 1990s, this parenting style is characterized by stronger regulation and the setting and upholding of rules. In this parenting style, parents actively set boundaries and explain with the goal of promoting adolescent's responsibility (De Winter, 2005, p. 11). Parents, who are urged to act as external frontal lobes, are expected to combine setting clear limits (strong control) with acknowledging and nurturing 
the needs of the adolescent (in this case: the needs of his or her brain). The teenage brain thus seems to function as credible support to this difficult balancing act. However, the emergence of two therapeutic repertoires - each with different action programmes and ideas of how and why to set limits - shows that the teenage brain does not give a conclusive answer but rather seems to be valued through this very style of authoritative parenting itself. Instead of challenging ways of parenting, the teenage brain thus reflects contemporary Dutch ideas of parenting.

At the same time, a clear discontinuity and moral change can be observed compared to earlier debates about science-based parenting. Some sceptics infer from the teenage brain a diminishing role for parents - resonating the debate around The Nurture Assumption by Judith Harris (1998). In this controversy, Harris argued that the formative role of parents on their children is grossly overestimated, at the expense of genetic factors and the role played by peers (Harris, 1998; Wesseling, 2004). In other words, a biological (in this case, genetic) view of child development would reduce parents' need to assert themselves. During the controversy surrounding Crone's revisions to her popular work, some critics of the teenage brain assumed a similar deterministic role of the teenage brain as a neurobiological explanation when they feared that it would lead parents to think that "they can't do anything about it" (Van der Heijden, 2013, para. 15). But, as we have seen, the story of the teenage brain shows that the teenage brain does not rule out the role of parents. Rather, the reverse is the case: it is mostly used to make an appeal to parents, as it comes with different and conflicting prescriptions of what to do, resulting in two therapeutic repertoires of parents-as-external-frontal-lobes. In contrast to the debate surrounding The Nurture Assumption, Crone's teenage brain allows for acknowledging biological characteristics of behaviour without leaving parents empty-handed. To accept the biological imperative of the teenage brain is to view adolescent behaviour as a result of a natural (and hence normal) process of brain development, a development that nonetheless requires specific actions and nourishment by parents.

\section{Parenting Turmoil: raising 'normal' adolescents}

The idea of parenting-as-external-frontal-lobe, including the notion of authoritative parenting (and the very concept of parenting style itself), is a procedural notion: It doesn't include a substantial, normative ideal of good parenting. In the two repertoires, what parents actually should teach their adolescents to 
become is not described in normative ideals (such as character building or responsible citizenship). Rather, the why of parenting is formulated in terms of protecting or stimulating teenage brains. Parenting teenage brains thereby seems a continuation of a shift that occurred already in the 1970s: just as parenting goals in the 1970s became formulated in terms of developmental psychology, current parenting goals are now formulated in terms of a natural and normal developing teenage brain.

The end-goal for many experts and parents using the teenage brain seems to be a 'normal' teenage brain, as a prerequisite for adulthood. However, as we have seen, what is considered 'normal' differed in the two repertoires. While normality in the first repertoire referred to the absence of abnormal behaviour or mental disorders, normality in the second repertoire referred to adhering to the norms of societal success. As many critics of the epidemic of ADHD, autism, dyslexia, and other mental disorders amongst children and adolescents have argued, the rise of such diagnoses indicates that the demands for being successful in society are increasing, and what counts as normal is shifting (Dehue, 2014; Horwitz, 2016). The second repertoire thus seems to respond to a moral change in parenting ideals since the 1970s: Normality is not only about an undisturbed natural development but has also become closely linked to contemporary ideals of societal success.

In a complex, dynamic society, where digital technologies challenge education, the job market, and other societal domains, and where social relations increasingly are maintained online, parents seem to feel anxious about the practical options they have for preparing their adolescents to successfully enter this society. In both repertoires, the teenage brain is presented as being shaped by external (societal) stimuli - for better or for worse. While the complex society itself is presented as beyond the scope of parenting, parents are not left emptyhanded. Parents cannot compete with the social and cultural imprinting of society on their teenage brains. But they are given the capability (and responsibility) of controlling or influencing the kind of stimuli their teenagers receive. As substitutes for their adolescents' brains, parents are encouraged to either shield their teenagers' brains from harmful stimuli or steer their teenagers towards acquiring the right stimuli. Parents in the second repertoire are reassured that the virtues for success in a meritocratic knowledge economy - creativity, flexibility, sociality - are already dormant in their teenage brains.

Extending adolescence from the age of eighteen to the age of twenty-two or twenty-five - for only then are the plastic brains of adolescents fully developed 
- seems a welcome answer and justification that adolescents just need more time to develop into responsible adults in our complex society (cf. Bloemink, 2011). Current Dutch trends such as delaying studies for gap years or living longer with parents before moving out are then seen as beneficial for the adolescent's developing brain (Bloemink, 2011). Whether this extension of adolescent becoming is also a blessing for parents, or rather a curse, is something that only external frontal lobes know. For some parents, knowledge of adolescent brain development seems to give relief. As this reader of Het Puberende Brein (2008) notes upon reflecting on the impact of Crone's book: "Already in the beginning of the book the penny dropped. It wasn't so much my teenager with his behaviour that caused 'problems,' the problem was that I didn't understand him!" (Ptries, 2013). In contrast to the relief experienced by Ptries, for some parents the teenage brain brings them insecurity, as this parent reflects after a workshop on the topic:

My child is not malleable and I have to stop projecting my opinions unto her. Or am I just a lazy parent who doesn't want to get the best out of her child, like one of the other parents snapped at me tonight? I struggle with this and I don't know what to do. (Marieke, 2012)

While Crone aimed to reassure parents with her knowledge of adolescent brain development, it remains in question whether the teenage brain actually leads to a reduction of parental anxiety and responsibilities in practice. It seems to frame the care and responsibility for a natural and successful adolescent brain development as the core parental responsibility from which other, derivative responsibilities follow. If only parents take care of the brains of their adolescents, they will become normal and flourishing individuals.

\section{Conclusion}

In this chapter, I have explored how the teenage brain is used to address parenting issues in Dutch parenting discourse. I traced valuations of the teenage brain through the value work of Eveline Crone to valuations of the teenage brain in parenting magazines, parenting books, and public discourse, where two different therapeutic repertoires of parenting as 'external frontal lobe' emerged. The plastic teenage brain, portrayed as 'unbalanced' and thereby open to environmental stimuli, affords different normative suggestions regard- 
ing expectations parents should have, the degree of control that should be exerted, and the way of exerting control. Together, these two therapeutic repertoires urge parents to both control and let go.

The core promise of the teenage brain (as articulated by both Crone and parenting experts) was that knowledge of adolescent brain development brings a new understanding of teenage behaviour - a new knowledge that would help parents in understanding, and thereby parenting, their adolescents. The teenage brain did not result in substantial changes of how parents should see their adolescents, or what it means to parent well. When I compared the teenage brain as parenting advice to earlier notions of adolescence and parenting, I did however find some shifts in the relative weight of views on adolescence and ideals of parenting.

As we have seen, a neurological understanding of adolescent behaviour did not result in 'spectacular insights' because it echoes traditional views of adolescents as facing the turmoil between the passions and the slow establishment of reason so vividly described by Aristotle and Hall. However, in contrast to the dominant idea of teenagers as 'defective adults,' a more positive understanding of teenagers emerged. While such a reading of adolescence as having a kind of 'vitality' already was scientifically described by Hall, there is now a richer, biological discourse for positively valuing the uniqueness of this phase in life. As a result, teenagers in Dutch parenting discourse are seen not only in negative light, as defective individuals, but also as unique individuals, as the emergence of the second repertoire indicates. Crone's attempt to elevate the negative stigma of adolescents can therefore be considered successful: These valuations found their way into the popular discourse of pedagogues and parenting experts.

As parenting advice, the teenage brain did not come with new ways to parent teenagers, or new parenting ideals. Rather, it seems to be evaluated through current Dutch ideals of parenting: protection by setting clear limits (strong control) while acknowledging and nurturing the needs of the adolescent for selfactualization. While the teenage brain does not give a conclusive answer to this balancing act of authoritative parenting, it does give this particular style of parenting an additional biological underpinning, by which it gains suggestive strength. Compared to earlier 'biological evidence' in parenting advice, knowledge of the teenage brain is different: It does not leave parents emptyhanded, but rather comes with its own prescriptions. 
Whereas throughout the $20^{\text {th }}$ century self-regulation through character building was a key parenting ideal, self-regulation now is relocated to the prefrontal cortex. As a result, the implicit end-goal when dealing with the teenage brain seems to be a normal development of the PFC. As we have seen, normality here takes the meaning of both an undisturbed natural development - free from 'abnormal,' medicalized mental disorders - and an adherence to the norms of success in a complex knowledge society. This latter ideal of normality reflects changes in parenting practices since the 1970s. Preparing adolescents for successfully entering a complex meritocratic society is a contemporary concern for parents. Through the plastic, teenage brain, the ambition of parents to be responsible for the societal success of their teenagers and the resulting anxiety of becoming 'control freaks' are both addressed by normalizing virtues that we seem to deem important in our current society, and naturalizing them in terms of the plastic, teenage brain.

In describing these positive capacities, parenting experts and pedagogues picture an ideal for our post-industrial, digital knowledge economy. In a society where we claim flexibility, creativity, sociality, and idealism as virtues, it seems that parents can learn something from their adolescents. The tragedy of adolescence (for adults at least) is that you seem to lose some of that vitality when growing up. The creative, social, and idealist adolescent reminds adults that adolescence can be seen as a period with valuable intrinsic qualities; It is not only about the balancing act of parenting. For parents, having become fullfledged, working adults, the unique traits that once came with this plastic stage of life are tragically no longer self-evident. Flexibility, creativity, sociality, and idealism suddenly appear not as a given but as a task. As we will see in the next chapter, these virtues of a plastic brain have to be maintained, practiced, and worked upon when we face the daily challenges of the contemporary workplace. 


\section{CHAPTER 3}

\section{A Monk at the Office: stress and the mindful brain in the workplace}

\section{Introduction}

T $t$ is that nagging feeling, lingering in the back of your mind. The cause of 1 those painful knobs in your shoulders, and it is probably the reason why you snarled at your teenage daughter at breakfast this morning. It's what some seem to wear proudly as a 'badge of honour' (Tan, 2012), but most of us wish we could do without: stress. Stress is occupational hazard number one; approximately one third of Dutch work-related absenteeism is said to be the result of stress (Ministerie van Sociale Zaken en Werkgelegenheid, 2013). Chronic stress can lead to all kinds of health problems, ranging from anxiety and heart diseases, to burnout, depression, and other mental illnesses. Instead of making our working lives easier, the introduction of digital technologies at the workplace, allowing us to respond to our colleagues' emails during evenings, weekends and holidays, seems to result in even more work-related stress, now dubbed technostress (Brod, 1984; Popma, 2012). Work-related stress seems to be the biggest obstacle to working and living well in our contemporary work culture.

In recent years, one peculiar antidote to stress has become increasingly popular: mindfulness, a form of meditation originated from Buddhist contempla- 
tive traditions. More and more corporations and organizations in Western affluent countries have started to encourage their employees to start meditating. While mindfulness-based stress reduction programmes (mbsr) took flight at Siliconvalley corporations such as Google, Twitter, and Facebook, the trend quickly trickled down to local (and less trendy) institutions such as KLM, Rabobank, Philips, universities, local municipalities, fire brigades, and even the Tax Office (Belastingdienst in the Netherlands). Advocates - including celebrities such as Arianna Huffington, Ruby Wax, Richard Branson, and in the Netherlands Sophie Hilbrandt - promise that mindfulness prevents stress at the workplace, increases productivity, and enhances well-being. In the Netherlands, trade and labour unions plead for mindfulness to become part of collective agreements ("Vakbonden willen meditatie in cao," 2008). Even business leaders at the top level are discussing how mindfulness is impacting the workplace, as mindfulness has been part of the programme of the World Economic Forum in Davos since 2013 (Gelles, 2015). Mindfulness is fast growing into a common practice at the workplace, and a standard element in the toolkit of HR managers.

This enthusiastic embrace of an age-old Buddhist practice by employees and managers - who normally talk about targets, efficiency and productivity seems a bit odd. How did mindfulness emerge as something desirable in the context of work? Taking a look at flyers of training programmes, one justification for this unlikely marriage stands out: the plastic brain. Training programmes and popular books on mindfulness invoke knowledge of the plastic brain to diagnose problems at the workplace and herald the benefits of meditation. Popular mindfulness coaches invite participants to take control of and change their brain functioning, promising new ways to prevent stress and achieve happiness and well-being. The combination of an age-old form of meditation with cutting-edge science draws considerable media attention, by which the idea of mindfulness as brain training - or what I will refer to as the mindful brain in this chapter - quickly entered Western mainstream culture, including the workplace. ${ }^{31}$

In this chapter, I explore how a neuroscience understanding of mindfulness is used to articulate and challenge ideas of working well. What kind of good employee do you become by practicing mindfulness? And precisely how did neuroscience knowledge enable the rise of mindfulness in the workplace of

\footnotetext{
31 With 'the mindful brain,' I do not refer solely to the eponymous book of Daniel Siegel (2007a) but rather to a neuroscientific understanding of mindfulness, including all mindfulness books and programs that somehow invoke knowledge of the brain to promote mindfulness.
} 
knowledge workers? ${ }^{32}$ How is this once-Buddhist practice of meditation made valuable as a desirable intervention for both employees and managers? I show how a neuroscience understanding of mindfulness allows management coaches and mindfulness advocates to turn mindfulness into a desirable intervention for dealing with a variety of concerns, challenges, and ideals at the modern workplace. As such, I regard the mindful brain as the latest intervention in the history of science-based management. By explicating how the contemporary work ethic is conceptualized in popular mindfulness programmes and books, I question to what extent mindfulness at the workplace challenges what it means to work, and how to do it well.

After a short historical sketch of shifts in the meaning of work throughout the history of science-based management, I explore in the third section of this chapter what value work neuroscientists employ to make mindfulness accessible to a general audience. By focusing on a group of neuroscientists who played a key role in popularizing a neuroscience understanding of mindfulness, I show how they make the plastic, mindful brain valuable by de-ethicizing it, turning it from a Buddhist practice into a form of brain training. It is this seemingly neutral mindful brain that enabled mindfulness to travel from the lab to the workplace. In the fourth section, I show how management consultants and other mindfulness advocates re-ethicize the mindful brain. As we will see, the plasticity of the mindful brain allows for different valuations of neuroscience knowledge in practice, resulting in two distinct therapeutic repertoires of working well. Finally, in the fifth section, I focus on the question of moral change: To what extent does mindfulness at the workplace destabilize the self-knowledge, options to act, ideals of working well, or outlook on challenges facing the contemporary employee and manager?

In the previous chapter on parenting, the susceptibility of the plastic, teenage brain was presented as a given and part of the problem for parents: how were they to deal with teenagers' openness to environmental stimuli? In the case of the adult, stressed-out brain, the changing brain appears not as a given or a problem but becomes a task and an ideal for workers to strive for. The stressed-out brain is presented as rigid, as suffering from a lack of plasticity. Plasticity here takes a different form: Instead of susceptibility to the environment, plasticity in the case of the mindful brain entails self-directed change. As we

\footnotetext{
${ }^{32}$ I focus in this chapter on a specific subset of work, namely the mental labour of knowledge workers in Western, affluent societies (notably the Netherlands and the U.S.).
} 
will see, it is through mindfulness that employees and managers alike are given the agency to overcome stress, by practicing their plastic brains.

\section{A History of Work Ethic and Science-based Management}

In today's society, work seems to be the central aspect of our adult lives. ${ }^{33}$ Most people work most of the time. Work offers income, structures your daily life, designates social status and economic class, and provides you with a topic to discuss with strangers at awkward parties. Apart from these extrinsic values, work today is often seen as having an intrinsic value: It is good to work, and to do it well. Work is valued by many as the most important means of selfexpression, self-determination, and cultivating self-respect; through work, we can flourish. But work hasn't always been considered so important in our lives. Therefore, before we discuss valuations of work in modern science-based management, we take a short look at pre-modern ideas about the meaning of work, as these provide a helpful contrast to how those meanings have changed since the advent of science-based management in the $20^{\text {th }}$ century. ${ }^{34}$

\section{Pre-Modern Views of Work: from slavish curse to religious vocation}

The Greeks were no great fans of work. For Plato and Aristotle, for example, the fleeting character of work would absorb and degrade the mind. The need to work to provide for economic needs, or to service a master, was denounced as slavish. Slaves would have to work, "in order that the minority, the élite, might

\footnotetext{
33 Throughout this chapter, I use the terms "work" and "labour" interchangeably, just as I use "workers" and "employees" to denote the mental labour of knowledge workers in Western, affluent knowledge economies.

${ }^{34}$ Perhaps needless to say, my historical sketch has some limitations. This overview is not meant to give a linear or exhaustive overview of the phenomenon of work and how it changed over time. Rather, it provides a broad introduction to changed meanings and valuations of the idea of work throughout history, which serves as a backdrop for understanding mindfulness as a science-based, managed intervention. Thus, instead of economic theory, labour mentalities, social classes, practices of work, work and gender, or specific local work contexts, I have focused on a broad intellectual history of the idea of work - encompassing disciplines such as social history, philosophy of work, business ethics, and history of management -emphasizing mental labour over physical labour wherever possible. While I use relevant critical secondary sources, I try to sidestep an a priori politicization of work in this section. For that reason, I have not included post-Marxists or critical theory sources, nor do I discuss recent characterizations of our contemporary work society in this section (cf. Barbrook, 2006; Boltanski \& Chiapello, 2005; Graeber, 2013; Florida, 2002; Sennett, 1999), as such characterizations are precisely the topic of my empirical analysis.
} 
engage in pure exercises of the mind - art, philosophy, and politics" (Tilgher, 1930, as cited in Hill, 1992). At the same time, Greek philosophers (especially the Stoics and Epicureans) valued self-discipline as one of the most important virtues. While Greek views on work are thus more ambiguous and advanced than I can portray here - both Plato and Aristotle admired shepherds and farmers, saw idleness as common vice, and considered politics and warfare as extrinsically good forms of work - work in general was not seen as having an intrinsic value, as it was considered a hindrance for the citizen's pursuit of virtue, and for the philosopher's quest for Beauty and Truth (Ehmer \& Lis, 2016; Hill, 1992). ${ }^{35}$

This negative valuation of work continued in the Judeo- and Christian traditions. In early Christendom, work is seen as a punishment from God for man's original sin: Man has been planted in the Garden of Eden to 'work it and care for it' (Hill, 1992; Van Luijk, 2011). Echoing the Greeks, the ideal occupation for Augustine for example was the virtuous life of the self-disciplined mind through contemplation and prayer (Hill, 1992). During the Renaissance, work gained appreciation, especially technical and creative craftsmanship. For Italian philosopher Pico della Mirandola, for example, man was his own maker, a homo faber (a "working man"), who's "work in the world is to create" (Sennett, 1999, p. 102). Self-discipline here became mixed with self-fashioning, the controlling of one's own life and fate. But this view was at odds with Catholic dogma. Work only became somewhat more positively valued after the Reformation. For the Calvinist, the mercy of God for his life of sin was a matter of fate; to be or not to be chosen for the afterlife remained a mystery. To reduce doubt and uncertainty about one's predestined chances to ascend to the afterlife, the only thing a Calvinist could do was to take care of God's gift to the best of one's abilities - and this meant pursuing a life of discipline and good work. Work gained a religious connotation, as it meant serving God and one's community: Work thus became a moral duty (Ehmer \& Lis, 2016; Hofmeester \& Moll-Murata, 2011; Van Luijk, 2011). As famously argued by Max Weber in Die protestantische Ethik und der Geist des Kapitalismus [The Protestant Ethic and the Spirit of Capitalism] (1905), a new moral relationship between people and their work emerged, defined in the German term Beruf: a religious vocation that demands fulfilment (Boltanski \& Chiapello, 2005). It is this idea of work as vocation, characterized by a diligent work ethic and a 'this-worldly' ascetic lifestyle, that

${ }^{35}$ For an elaborate view on the ambiguous role of work in Greek culture, see Ehmer \& Lis (2016). 
resulted in an accumulation of wealth that would lay the groundwork of modern Western capitalism, as the famous thesis of Weber goes.

\section{Science-Based Management: from efficiency to personal fulfilment}

In the era of the Enlightenment, attitudes towards work and labour diversified and changed. Next to religious vocation, work in the $18^{\text {th }}$ century became conceptualized as both a commodity and the foundation of civil society (Ehmer \& Lis, 2016, p. 20). Mercantilist views on economic growth - which are based on the maximization of gold and silver through trade - became criticized and slowly replaced by industrial capitalist approaches. British political economists such as Adam Smith argued that the pursuit of self-interest is the key driver of economic growth, and that this pursuit requires a division and specialization of labour. Differentiation in tasks, together with high wages and sufficient income, would increase the efficacy and industriousness of workmen (Anthony, 1977, p. 54; Ehmer \& Lies, 2016, p. 20). True to enlightenment ideals, this division of labour was also seen as a desirable social process, as a basis for human cooperation which would progress mankind and society. Such a view included propagating work as pleasurable and delightful (Ehmer \& Lis, 2016, p. 20).

In the $19^{\text {th }}$ century, Smith's ideas about the division of labour materialized in the emergence of factories and large, mass-production workplaces. As capitalism flourished and the scale of business grew, especially after the second Industrial Revolution in the 1860s, a new breed of employees of mental labour emerged: the "labour aristocrats," well-trained engineers who took on managerial tasks (Anthony, 1977, p. 64). Planning and control through the specialization of tasks and the professionalization of managers would replace the paternalism of the employer towards "lazy and disobedient" workers (Anthony, 1977, p. 76; Hill, 1992). Work increasingly became secularized and specialized. The introduction of scientific management, with Ford's assembly lines, Taylor's specialized division of labour, Fayol's administrative management, and Weber's formal bureaucratic hierarchy, lead to a standardization of roles, rules, regulations, and measurement systems. A rational, scientific method to business would improve productivity and efficiency, and for the worker it would result in increased pay - monetary gains were seen as the motivating force of employees (Hill, 1992).

However, a detailed division of labour had a human cost, as Adam Smith already foresaw: performing the same task over and over again makes a worker "as stupid and ignorant as it is possible for a human creature to become" 
(Smith, 1776 [1976]: V.1.178). Partly due to resistance to this 'alienating,' 'disenchanted,' and 'meaningless' workplace which resulted in strikes and the rise of labour unions, management theories around WWII shifted towards behaviourist models, where the idea of an employee solely driven by monetary gains was replaced by a social model of the employee as one motivated by the fulfilment of (social) needs. The idea of work as vocation changed. In Western countries such as the U.S., the justification for work as a calling or vocation became secularized and replaced with the idea of public usefulness. Next to discipline and obedience came virtues such as ambition and becoming 'respectable' as parts of work ethic (Anthony, 1977, p. 74; Hill, 1992). Books emerged for workers that gave advice on how to become successful and gain material wealth and moral virtue through hard and disciplined work (Anthony, 1977, p. 78; Wren \& Bedeian, 2008). Work came to be seen not only as a necessity or duty but as a blessing and a good in itself (Anthony, 1977, p. 78).

In the second half of the $20^{\text {th }}$ century, management as industry and scholarly discipline boomed, resulting in a plethora of management theories, journals, and organizational models, along with management specialists and gurus such as Mary Parker Follet and Peter Drucker - the latter well-known for his coining of the term "knowledge work" (Drucker, 1946). In Western countries, the labour market and occupational structure gradually shifted from industrial environments towards service and knowledge occupations (Väänänen et al., 2012). As management and work became a scholarly subject, and the educational levels of the workforce increased, the workplace became more and more scientizied, with interventions such as operations management, management by objectives, and reengineering, and organizational theories such as transaction cost theory or resource dependence theory, serving as well-known examples (McGrath, 2014; McKinley \& Mone, 2003).

Since the 1960s and 1970s, the shift towards a service and information economy came with a change of management approaches. No longer dictated by the industrial factory machines, the needs of the knowledge worker and manager in the information society changed. In the 1960s, coaching and human relations became an important task of managers, and management was characterized by increasing the autonomy of workers through decentralisation; career opportunities and job security were seen as important motivations for employees (Boltanski \& Chiapello, 2005, p. 88). Work-related stress emerged as an individual problem in the work environment, and the social and psychological dimension of workers became basic features in management models of the 1970s and 
1980s. The ideal of a factory worker able to routinely perform tasks changed to the ideal of a "multi-skilled, emotionally sensitive team worker with the skill of mental adaptation" (Väänänen et al., 2012, p. 792).

Neo-management models in the 1990s focused on team work, short-term projects, precariousness of contracts, and employees' self-knowledge and personal fulfilment (Boltanski \& Chiapello, 2005; Sennett, 1999). Managers are conceptualized as coaches, visionaries, or leaders, who assert themselves not through their position in the organization but through personal qualities such as their human relations skills and charisma (Boltanski \& Chiapello, 2005). As Sennett (1999) famously showed, in this emerging "flexible capitalism," the social and psycho-emotional character of the employee is central to modern work life (Sennett, 1999). Work-related stress came to be seen more and more as a public and mental health problem, one that was an issue for management (Väänänen et al., 2012).

This short historical overview of ideas of work and management shows that work throughout history has been valued differently: from curse, moral duty and religious vocation, to commodity, a foundation for civil society, economic necessity, and a means for social success, and later, for personal fulfilment. Management techniques parallel societal and economic developments. Since the 1990s, challenges that face corporate management include the rise of digital technologies and globalized networks of commerce. There seems to be a consensus in modern management that these developments are transforming the nature of work, as "average actual working time has become more intense, faster paced, more flexible and longer" (Ehmer \& Lis, 2016, p. 1). This has led to a multitude of characterizations of our contemporary work society; propagations of "new" work ethics and classes of workers abound (cf. Barbrook, 2006; Boltanski \& Chiapello, 2005; Florida, 2002; Graeber, 2013). The meaning of contemporary work, and how to do it well, have again become important questions for contemporary employees and managers alike. It is in this context that the mindful brain appears as a popular intervention at the workplace. Just as Weber took the Catholic monk out of the monastery and into in the world to show how ascesis was essential for the rise of capitalism, so too does neuroscience bring the Buddhist monk to the work floor. Before we can explore whether this leads to a new work ethic, we first have to look at how mindfulness meditation was made accessible to non-Buddhist audiences as a science-based programme in the first place. 


\section{The Mindful Brain: from Buddhist practice to brain training}

During the 2005 annual meeting of the Society for Neuroscience (SfN) in Washington (U.S.), a peculiar guest addressed the world's largest gathering of neuroscientists. No other than His Holiness the Dalai Lama, the 14th incarnation of the Living Buddha of Compassion, gave a speech about the neuroscience of meditation. The appearance of the Buddhist leader at the SfN conference was not coincidental. Since 1992, the Dalai Lama had helped neuroscientists conduct research on meditating monks in his residence in Dharamsala. ${ }^{36}$ Over the past two decades, a group of enthusiastic neuroscientists has emerged, who - with the help of the Dalai Lama - has been focused on researching and propagating the effects of mindfulness meditation on the brain. At the conference, the Dalai Lama spoke of the effects of mindfulness and mental training on the plasticity of the brain, and he pleaded for a closer collaboration between neuroscience and the Buddhist tradition.

For many years, a presentation about meditation at a (neuro)science conference (let alone one made by a religious leader) was would have been unthinkable - and for some, it still is. ${ }^{37}$ Meditation as an object of scientific study has long been met with scepticism. Apart from some psychological studies in the 1970s and 1980s, research on mindfulness and meditation was scarce, and its impact was limited to a small group of scholars (Lutz, Dunne, \& Davidson, 2007). In 1979, molecular biologist Jon Kabat-Zinn (University of Massachusetts Medical Center, U.S.) decided to turn the meditation lessons of his Zen teacher into a practice to help people cope with chronic pain (Kabat-Zinn, 1982). This practice became known in the West as mindfulness. Kabat-Zinn describes mindfulness as "the awareness that emerges through paying attention on purpose, in the present moment, and nonjudgmentally to the unfolding of experience mo-

\footnotetext{
${ }_{36}$ Together with entrepreneur Adam Engle and the neuroscientist and philosopher Francisco Varela, the Dalai Lama founded the Mind and Life Institute in 1987, whose yearly conference aims to foster collaboration between neuroscientists and meditators.

${ }^{37}$ In the month preceding the annual conference of SfN at which the Dalai Lama would speak, a small controversy broke out amongst brain researchers. Some neuroscientists argued that science, religion, and politics shouldn't be mixed, and they feared that the public credibility of the field of neuroscience was at stake when a religious leader would talk about meditation and the brain (Carey, 2005). In a petition, the protesters further argued that existing studies on meditation and the brain lacked scientific rigor and objectivity, and that the topic of the meditating brain should thus not be part of the conference (Adam, 2005). Supporters of the invitation of the Dalai Lama accused the mostly Chinese criticasters in turn of being politically motivated. Eventually, there was hardly any protest during the conference, and the talk of the Dalai Lama was met with enthusiasm (Bhattacharjee, 2005)
} 
ment by moment" (Kabat-Zinn, 2003, p. 145-146). In this popular definition, mindfulness is the training of attention by focusing on the present and becoming aware of how thoughts, emotions, and bodily sensations emerge - without being judgmental. The mindfulness programme of Kabat-Zinn, including his definition of mindfulness, became known as 'mindfulness-based stress reduction' or mbsr (Kabat-Zinn, 1990). ${ }^{38}$

Since the 1990s, the on-going work of Kabat-Zinn and the Dalai Lama's Mind and Life Institute began to attract more attention from psychologists and neuroscientists, and mindfulness and meditation became topics deemed worthy of scientific inquiry. ${ }^{39}$ The rise of fMRI scanning techniques accelerated neuroscience research on mindfulness and meditation in the 2000s. A group of enthusiastic neuroscientists emerged who started researching and propagating the effects of mindfulness meditation on the brain, soon dubbed contemplative neuroscience. Next to Kabat-Zinn, scholars such as psychologist Richard Davidson (founder of the Center for Healthy Minds at the University of Wisconsin-Madison, U.S.), cognitive scientist Antoine Lutz (Waisman Lab for Brain Imaging \& Behavior at the University of Wisconsin-Madison, U.S.), psychologist and science journalist Daniel Goleman, and many others have been influential in establishing a neuroscience-based understanding of mindfulness, and in making their research valuable to both general and professional audiences. In the following section, I introduce some characteristics of a neuroscientific understanding of mindfulness meditation by focusing on the work of these key scholars. ${ }^{40}$

\footnotetext{
${ }^{38}$ Kabat-Zinn offers these programs in his Stress Reduction Clinic at the University of Massachusetts Medical School (U.S.). He is no longer the only one. In recent years, mindfulness-based cognitive therapy (mbct) as therapeutic intervention has become a widely endorsed and accepted method for treating burnout and stress in the medical realm, as well as to alleviate pain or as an alternative to antidepressants. I focus on this chapter not on the clinical use of mindfulness, but rather in the use of mindfulness in the more mundane context of work.

39 There are many different forms and traditions of (Buddhist) meditation practices, of which mindfulness can be seen as a specific one. Often, neuroscience studies focus on a particular form of meditation, which later (when disseminated) becomes described as mindfulness. In this sense, mindfulness has become a popular metaphor for a variety of meditation practices.

${ }^{40}$ To introduce what a neuroscientific understanding of mindfulness entails, and how scholars in contemplative neuroscience valorise their work, I chose to focus on international key players, instead of limiting my material to the few Dutch researchers on mindfulness. The reason for this, is that these international scholars have paved the way for the widespread valuation of mindfulness as brain training in all kinds of societal contexts, in the U.S. and abroad. Moreover, their work is frequently featured and evaluated in Dutch mindfulness books, courses, and public discourse. The strategies of value work that these scholars employ, are similar to those that Dutch mindfulness advocates employ when making the mindful brain valuable in the local (Dutch) context of work (as we will see in the next section).
} 


\section{Popularizing Contemplative Neuroscience: introducing the mindful brain}

Neuroscience research into the effects of (mindfulness) meditation on the brain from scholars surrounding Davidson, Lutz, and Kabat-Zinn drew a lot of media attention. Featured in international and Dutch newspapers and magazines, their views found their way into all kinds of mindfulness interventions, as we will see later. In 2003 Davidson, Kabat-Zinn, and their colleagues analysed stressed brains and the immune systems of employees of a bio-tech company before and after an eight-week mbsr mindfulness programme (Davidson et al, 2003). The authors found that "meditation can produce increases in relative left-sided anterior activation that are associated with reductions in anxiety and negative affect and increases in positive affect" (Davidson et al, 2003, p. 11). The authors use positive affect as an important indicator of meditation, by which mindfulness meditation in study becomes defined as a form of emotion regulation.

In a similar vein, in their 2004 study, Lutz, Dunn, \& Davidson measured with EEG the brains of long-term Buddhist practitioners with over 10,000 hours of meditation training and compared them with the brains of a group of volunteers. Subjects were instructed to engage in objectless meditation, focusing on loving-kindness and compassion. The researchers found a significant increase of gamma activity of the brains of the Buddhist meditators. The authors equate meditation with mental training and link it to high-order cognitive, attention, and affective processes which "are flexible skills that can be trained" (Lutz et al., 2004, p. 5). Gamma-band synchrony becomes associated with awareness, as this neural mechanism is said to reflect "a change in the quality of moment-tomoment awareness, as claimed by the Buddhist practitioners" (Lutz et al, 2004, p. 5). The authors conclude that this form of meditation involves temporal integrative mechanisms and can induce both short-term and long-term neural changes (Lutz et. al, 2004). This study, published in PNAS, became a widely used example of the scientific proof of meditation's effects and of its potential to change the brain for the better.

In 2008, Lutz, Davidson et al (2008) studied the effects of a loving-kindness compassion meditation on the affective processing of the brain. The researchers compared the brain activity of meditating novices with meditation experts and found that expert meditators showed increased activity in the insula and an increase in the temporal parietal juncture (the right hemisphere) during presentation of negative sounds while meditating - the authors link both areas to the processing of empathy and emotion sharing. They conclude that cultivating 
positive emotion (through compassion meditation) alters the activation of the insula and cingulate cortices in the limbic region, circuitries which are linked to empathy (Lutz et al., 2008).

The studies of Davidson and his colleagues are obviously not exhaustive of the wide variety of neuroscience and mindfulness marriages in popular culture -these few authors do not even begin to represent the whole emerging field of contemplative neuroscience. ${ }^{41}$ However, without overly simplifying the stillincreasing amount of neuroscience research on meditation, these influential works do show us some broadly-shared tenets of a neuroscience understanding of mindfulness. In these examples, aspects of mindfulness become linked to a specific brain function: emotion regulation, attention control, and cognitive performance, for example. But the most important message that these studies portray, and one which invites a variety of valuations of the mindful brain, is that mindfulness meditation has a measurable effect on the functions and/or structure of the brain, and that this effect can be trained. In a neuroscientific understanding of mindfulness meditation, mindfulness becomes defined as the training of specific brain traits.

\section{Making the Mindful Brain Valuable: de-ethicizing mindfulness}

The 'hard' knowledge of the neurosciences has made mindfulness acceptable as a scientific object, both for lay audiences and experts alike (Tresch, 2011). However, as we already saw in the previous chapter, such (neuro)scientization is not a self-evident process: It requires the deliberate articulation and alignment of different kinds of values - efforts I have labelled value work. Just as in the case of the teenage brain, (neuro)scientists who study mindfulness aim to make their research and claims accessible and valuable by mobilizing concerns and promises, and by guiding the normative uptake of their claims to both a general audience and a professional audience - in this case, managers and employees.

\footnotetext{
${ }^{41}$ There are of course many more research groups and scholars studying meditation and mindfulness in the brain, linking mindfulness to a wide range of mental states, brain functions and areas. To name only a few examples - as the number of neuroscience studies on mindfulness is almost exponentially increasing every year - studies have linked mindfulness to emotion regulation (Davidson et al., 2003), attention control and cognitive performance (Lutz et. al, 2004), happiness (Lutz et al., 2008), social anxiety (Goldin \& Gross, 2010), stress (Tang et al., 2007; Creswell et al, 2014), selfawareness (Dor-Ziderman et al., 2013; Josipovic, 2014), academic performance (Tang et al., 2014), and pain regulation (Gard, Hölzel, Sack, Hempel, Lazar, Vaitl, et al., 2012). In the Netherlands, Radboud Universitair Medisch Centrum voor Mindfulness conducts (neuroscience) research on mindfulness.
} 
The most important part of value work by neuroscientists and other popularisers of the mindful brain is coordinating the normative uptake of the research. Studies on meditation and mindfulness - including those by Davidson and his colleagues - are not uncontroversial. ${ }^{42}$ For example, in a radio show, Kabat-Zinn reflects on how he made mindfulness accessible to a wider audience:

Because it's often freighted, even the word "meditation" is freighted with so much cultural baggage and so much, really ideological baggage and sort of belief baggage that the essential beauty of it is often really not apparent to people until long after they have somehow wandered into the domain of it. (...) And so I tried to create a kind of glide path into meditation that would be so common-sensical and accessible and based on what people really need and also fear and are challenged by, that we could at least empirically test whether, if it was framed in that kind of way, regular mainstream Americans would take to meditation. (Kabat-Zinn, as cited in Tippett, 2012)

The "ideological and belief baggage" Kabat-Zinn refers to is the Buddhist context of mindfulness. In Buddhist traditions, especially Theravada Buddhism, mindfulness is generally considered to be a form of insight-meditation or vipassana, in contrast to forms of meditation focused on concentration. According to the Dalai Lama, meditation in these traditions is described as bhavana ('cultivation' in Sanskrit) or gom ('cultivating habits or familiarity' in Tibetan) (Dalai Lama, 2005). Mindfulness, often described by Buddhist scholars as the heart of Buddhist meditation, is about cultivation, especially the cultivation of the habit of attention as a means for gaining deep insights into the laws or nature of the mind, emotion, and suffering. In Buddhist ontology, suffering is part of our human condition, as humans typically suffer from three destructive emotions, or "poisonous diseases": greed, hatred, and ignorance (or unawareness). To focus attention inwards through meditation ideally results in a 'deeper' understanding of these emotions and thoughts. The goal is to find ways to transform ourselves so that a more 'fulfilling' or 'wholesome' way of being can be found (Dalai Lama, 2005). Mediation practices in the Buddhist contemplative tradition are there-

42 There is, for example, discussion about the methodological quality and lack of replication of results (Tang, Hölzel, \& Posner, 2015). Furthermore, because many influential neuroscience advocates of mindfulness (including Davidson, Lutz and others) are enthusiastic meditators themselves, their solely positive affirmation of mindfulness has been questioned (Tang, Hölzel, \& Posner, 2015). For a recent overview of critiques on neuroscience research on meditation, see Van Dam, van Vugt, Vago, Schmalzl, Saron, Olendzki ... \& Meyer, 2017). 
fore aimed at training values such as compassion, empathy, tolerance, kindness, and happiness, as they help to prevent and overcome the destructive emotions. Metaphysically or spiritually, mindfulness can be described as "an attempt to appreciate the deep mystery of being alive and to acknowledge being vitally connected to all that exists" (Kabat-Zinn, 2001, p.24). Mindfulness is traditionally part of a wider conceptual, ethical, and metaphysical framework, including the ethical prescript of non-harm, the moral imperative to engage in virtuous action, and the disciplined training of the mind to prevent suffering and to increase compassion and kindness (Goleman, 2003; Kabat-Zinn, 2003, p.146).

Advocates of the mindful brain coordinate the uptake of mindfulness by dismantling it from this traditional context and de-ethicizing it. Scholars such as Kabat-Zinn and Davidson employ two tactics for de-ethicizing mindfulness: displacing the Buddhist context of mindfulness, and translating Buddhist values onto the brain. For example, Kabat-Zinn argues that "there is nothing particularly Buddhist" about mindfulness and instead describes the practice as an "inherent human capacity," a "deep inquiry" having to do with attention and awareness as universal human qualities (Kabat-Zinn, 2001, p. 15; Kabat-Zinn, 2003, pp. 145-146). In these examples, Kabat-Zinn values mindfulness as not specifically Buddhist, religious, or philosophical but rather as having certain epistemic values. The goal of this tactic of displacing Buddhist context and connotations and replacing them with scientific ones is to show that Buddhism and science are more similar than different. ${ }^{43}$ Such an anti-metaphysical and empirical reading of Buddhism, a recurring theme in Western society, has some benefits: It makes the practice compatible with atheism and other religions, and it makes research eligible for public funding (Lopez, 2008; Tresch, 2011; Sun, 2014). And it allows neuroscience to engage with meditation as research object. The foregrounding of epistemic values of mindfulness, while displacing the

\footnotetext{
${ }^{43}$ Mindfulness advocates go to great lengths to draw similarities between the two domains. For example, the Dalai Lama often talks and writes about the commonalities between the two 'traditions,' reporting "a deep suspicion of any notion of absolutes," situating explanations in terms of the natural laws of cause and effect, and maintaining a methodological focus on empiricism (Dalai Lama, 2005). Other Buddhist advocates talk about a shared attitude of rationality, empiricism, scepticism and pragmatism, or to a shared goal of "critically (and not dogmatically) establishing the existence of universal laws" (Begley, 2009, p.11-13). As a consequence, the community of professional Buddhist meditators can provide (neuro)scientists with a unique "experiment of nature": the practices and teachings of Buddhist monks are then seen as a complementary resource, a 'phenomenology of the mind'. Buddhism can offer its experiential knowledge on the nature of emotion, suffering, and happiness, and its practical techniques of meditation, to inform studies in cognitive science and neurophenomenology (Goleman, 1995; Dalai Lama, 2005; Kabat-Zinn, 2003; Siegel, 2007b).
} 
spiritual and metaphysical connotations ("enlightenment") and the ethical context of virtuous living and non-harm, is a recurring and particularly successful form of value work, also when evaluating mindfulness in the context of work, as we will see later.

Only after doing away with its metaphysical, religious and spiritual connotations can mindfulness be established as a neutral, non-spiritual form of mental training. The second tactic for de-ethicizing mindfulness is further aligning mindfulness with science by translating or redefining Buddhist concepts and values in terms of the brain. In order to make mindfulness fit the neuro-lab and to study the effects of mindfulness and meditation on the brain, the 'holistic' practice of mindfulness is split into separate, measurable mental states and functions. Values such as compassion, empathy, and well-being become described in terms of specific brain functions, and Buddhist notions such as selftransformation and unawareness receive a neuroscience equivalent.

For example, in the previously introduced studies of Davidson and his colleagues, awareness is associated with gamma-band synchrony (Davidson et al, 2003), and happiness, compassion, and empathy are linked to the insula and cingulate cortices in the limbic region (Davidson \& Lutz, 2008). While authors portray a nuanced and limited definition of these concepts and values in their research articles, the (moral) connotations of these terms are re-introduced when their results are communicated to a wider audience. In the press release for Davidson and Lutz's (2008) study, in which they found that expert meditators showed increased activity in the insula and an increase in the temporal parietal juncture, the conclusion is translated and linked to both happiness and compassion: "The findings support Davidson and Lutz's working assumption that through training, people can develop skills that promote happiness and compassion" (Land, 2008). As Davidson states in a university news item and press release: "People are not just stuck at their respective set points [of happiness and compassion]. We can take advantage of our brain's plasticity and train it to enhance these qualities" (Land, 2008). Compassion, empathy and wellbeing are no longer described as Buddhist or secular ideals to strive for, but become naturalized, attainable qualities of the brain. 


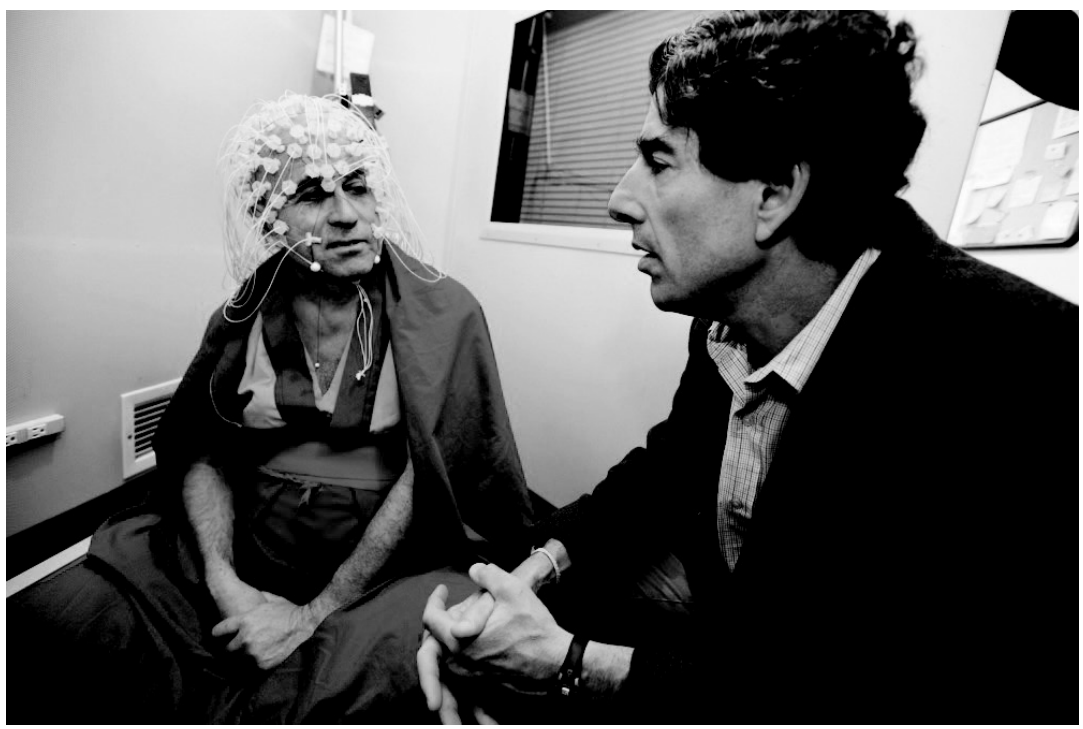

Figure 5: The French genetic scientist and Buddhist monk Matthieu Ricard (left), and Richard Davidson (right). Ricard, one of the co-authors and participants in Davidson \& Lutz's study (2008), became widely portrayed in popular media as "the happiest man in the world," as his test-score in the study was an outlier (Bates, 2012). Photo credit: Jeff Miller, WisconsininNews, June 2008.

Brain plasticity plays a key role in redefining Buddhist notions into neuroscience terms. Brain plasticity is mobilized not only as proof that meditation does have real, biological effects but also as an umbrella under which meditation becomes "fundamentally no different than other forms of skill acquisition that can induce plastic changes in the brain" (Davidson \& Lutz, 2008). ${ }^{44}$ This form of plasticity thus attributes to the individual subject the agency to change the brain. The idea of self-transformation - the goal of mindfulness in Buddhist teachings - has received a neuroscience equivalent in the concept of brain plasticity. It is the brain that "can be transformed through engagement with purely mental practices derived from the world's great religious traditions" (Goog-

${ }^{44}$ Over the past years, mindfulness and/or meditation has been associated with all kinds of structural and functional changes of the brain: with structural changes in the left prefrontal cortex (Kabat-Zinn, 2003), increased prefrontal cortical thickness (Grant et al., 2010; Lazar et al., 2005), increased activation of the insula and cingulate cortices (Lutz et al., 2008; Tang et al., 2010), structural changes to the amygdala (Hölzel et al., 2010), increased grey matter density and volume (Farb, et al., 2013; Hölzel et al., 2011; Pagnoni \& Cekic, 2007; Pickut et al., 2013; Vestergaard-Poulsen et al, 2009), and hippocampal volume (Wells et al., 2013). 
leTechTalks, 2009).45 As this last quote shows, through redefining Buddhist notions in terms of the brain, some connotations of the Buddhist roots of mindfulness are re-introduced. By carefully balancing scientific novelty with the idiom of an age-old contemplative tradition, continuity between modern neuroscience and ancient Buddhist traditions is enacted.

Through these tactics of de-ethicizing mindfulness - displacing the Buddhist context and translating values onto the brain - the meaning of mindfulness changes: what was once a Buddhist practice becomes the scientific training of particular brain traits. Once mindfulness is presented as brain training, mindfulness advocates can more easily appeal to concerns of non-Buddhists and propose mindfulness as a promising solution - the other two strategies of value work - without having to anticipate conflicts on the perceived religiousness or spirituality of mindfulness. The great promise of the mindful brain is that mindfulness is a neutral form of brain training by which real, biological changes in the brain can be achieved. The diagnosis is that happiness and human suffering (including that induced by work-related stress) are neither fixed entities nor based on luck, fate, or belief. Instead, the mindful brain entails that suffering is a problem of brain physiology, and that this suffering can be overcome by practicing mindfulness as brain training (action programme).

Many researchers on the neuroscience of mindfulness make their work valuable for specific audiences - and managers, employees, and businesses are a popular target group. For example, the work programme 'Cultivating WellBeing in the Workplace' at the Wisconsin School of Business (U.S.), affiliated with and based on the work of Richard Davidson, promises "to improve focus, resilience, and well-being to adapt to challenges in complex work environments" (Center for Advanced Studies in Business, 2017). These challenges include concerns such as stress, productivity, multitasking, and dealing with email and smartphones (Center for Advanced Studies in Business, 2017; Davidson, 2015). The programme, targeted at managers and employees, promises to "increase focus and strengthen connections in the brain related to executive function and goal-directed behavior," which is said to result in better focus and decision making and more compassionate managers (Davidson, 2015). Similar

\footnotetext{
${ }^{45}$ Brain plasticity is also used by Buddhist scholars to validate the Buddhist teachings that suffering can indeed be overcome, and happiness is within our reach: "Buddhism's is a story of how we are in pain and suffering and how we have the power to change that. The scientific findings about neuroplasticity parallel the Buddhist narrative of enlightenment because they show that, although we have deeply ingrained ways of thinking and although the brain comes with some hardwiring, we also have the possibility of changing" (Buddhist scholar Cho, as cited in Begley, 2009, p.14).
} 
concerns, diagnoses, ideals, and action programmes are mobilized in the evaluation of the mindful brain in the (Dutch) context of work, as we will see in the next section.

Mindfulness as brain training proves to be a very convincing and sexy topic. It quickly trickled down in popular discourse - specifically in management and coaching discourse. Presented as fresh from the frontiers of science and served with a ring of ancient Buddhist wisdom, the entanglement of Buddhist values and brain facts becomes a powerful normative and explanatory model for understanding contemporary concerns such as stress. The work of Davidson and his colleagues (amongst others) ended up in all kinds of mindfulness programmes, used to promote 'science-based' benefits of mindfulness for the brain. Once dislodged from its Buddhist context, turned into brain-training, and deethicized, the mindful brain appears malleable and versatile enough to be adapted to a variety of societal contexts, including the corporate world of office work.

\section{The Mindful Brain at the Office}

In the context of work, the mindful brain found a willing audience in boardrooms and open bullpen offices. Inspired by the work of scholars such as KabatZinn, Davidson, and many others, a variety of mindfulness programmes emerged in the past decade, to cater to the needs of employees and managers. Popular programmes and books include Google's Chade-Meng Tan's Search Inside Yourself (2015), David Rock's Your Brain at Work (2009), Michael Chaskalson's The Mindful Workplace (2011), Daniel Goleman's Focus: The Hidden Driver of Excellence (2013b), or The Potential Project's One Second Ahead: Enhance Your Performance at Work with Mindfulness (Houagaard, Carter, \& Coutts, 2016). ${ }^{46}$ Mindfulness has become a business, with consultancies offering expen-

\footnotetext{
${ }^{46}$ Just as in the previous chapter, I focus primarily on the public debate and discourse on the mindful brain in the context of work in the Netherlands, including the uptake of a neuroscientific understanding of mindfulness in the field of management and self-help for employees and managers. I first conducted a LexisNexis search on "mindfulness + hersenen" and "mindfulness + brein," between 2000 and 2015. This resulted in 280 articles. After removing duplicated articles and those that didn't discuss stress or other work-related issues, the sample consisted of 167 news media articles. In addition to media coverage on neuroscience and mindfulness, my empirical material includes Dutch popular management books such as Van der Roest's Mindful Leidinggeven (2013), DeWulf's Heartful Leven, Mindful Werken (2014), and Koole's Mindful Werken (2013). I included these books (from both U.S. and U.K. authors) because they were translated into Dutch or were featured in
} 
sive, tailored, in-house programmes for corporations and organizations, extensive eight-week management courses, self-help manuals, and mindfulness smartphone apps. As I will show, a neuroscience understanding of mindfulness allows consultants, coaches, managers, and other mindfulness advocates to make mindfulness compatible with a range of concerns, needs, goals, and ideals of both employees and managers.

Mindfulness advocates in the context of the workplace employ strategies of value work that are similar to the ones we saw with the researchers in the previous section. In the valuation of the mindful brain in this context, advocates articulate specific concerns facing the modern-day employee and manager and promise to demonstrate how specific ideals of working well can be achieved through mindfulness as brain training. However, while researchers on mindfulness focused on de-ethicizing mindfulness and making it compatible with contemporary science, advocates in the context of work re-ethicize mindfulness again by aligning the mindful brain with different context-specific values of working well. Based on the analysis of the valuation of the mindful brain in a variety of Dutch mindfulness programmes, books, consultants' websites, and public discourse, two recurring sets of moral argumentation on the plastic, mindful brain emerged. Each of these therapeutic repertoires of working well with a mindful brain consists of its own set of concerns, diagnoses, action programmes, and ideals.

In both repertoires of the mindful brain at work, the concern of stress is framed as a brain problem in order to propose mindfulness as brain training as a solution. The main diagnosis in both repertoires is that stress is the result of an (evolutionary) imbalance between different parts of the brain. To diagnose stress as a brain deficit, authors build upon research that conceptualizes the brain as consisting of different, sometimes competing parts or networks, such as dual process theories of mind (cf. Kahneman, 2011; Ledoux, 2002), or the theory of the triune brain (cf. MacLean, 1990; Siegel, 2007). ${ }^{47}$ In both repertoires, the

Dutch newspapers, magazines, and mindfulness books. The empirical material in this chapter further consists of mindfulness brochures, websites, and program booklets from a variety of (Dutch) consultancies and mindfulness coaches.

${ }^{47}$ In this (controversial) model the brain consists of three separate parts, differentiated by their role in the process of evolution of the human species: the brain stem, the limbic system, and the cortex. The brain stem, including the basal ganglia (the sympathetic system, "reptilian brain" or "monkey brain"), is considered to be the oldest part of the brain, responsible for instinctive behaviour (fight/flight responses). The limbic system (parasympathetic system or the "paleomammalian brain"), including brain regions such as the amygdala, hippocampus, and insula, is said to be responsible for our emotional processing. The neomammalian part, with its prefrontal cortex, is the 
plastic, mindful brain is heralded as a solution that counters this evolutionary imbalance or rigidity by increasing awareness of these workings of the brain; as a result, "you won't be forced to unconsciously default to primitive brain autopilot responses and actions" (Alidina \& Adams, 2014, p.29). While this diagnosis is recurring in both repertoires, different aspects are emphasized, with different plastic brains, action programmes of mindfulness as brain training, and different justifications for practicing mindfulness at the workspace.

\section{Therapeutic Repertoire I: the mindful brain as cognitive enhancer}

The central concern of employees and managers in this repertoire is the influence of digital technologies on contemporary working practices. The continuous distractions and bombardment of information through smartphones and email, and the accompanied high work pace and working style of multitasking, are major concerns for contemporary employees, freelancers, and managers alike, who fear a loss of productivity and a burnout through stress. How can we prevent stress and avoid "becoming a plaything of all these stimuli" (Van der Roest, 2013, p. 88)? How can we regain control and work effectively in such a work environment? Mindfulness is presented in this repertoire as a necessary competency for a digitized workplace.

The main diagnosis in this repertoire is that our brains are not suited for the 21st century workplace, with its high technology and fast pace. The distractions of the digital workplace are put forward here as resulting in a 'brain overload': a state of stress that is deemed unnatural, since our brain "hasn't had time to properly evolve" in relation to new screen technologies (Alidina \& Adams, 2014, p. 152). Mindfulness advocates especially take aim at the common practice of multitasking. We read in popular mindfulness books that our brains are "just not equipped for multitasking" (Wax, 2013, p. 7), which forces our brains into "a higher state of alert," one of "permanent crisis" (Van der Roest, 2013, p. 25). Digital technologies and multitasking make us inefficient and stressed as we suffer from "the frontal assault on our focusing ability from the mounting tide of distractions" (Goleman, 2013b, p. 23). Brain overload is explained as a result of the fight/flight response: the ruling of the parasympathetic and sympathetic brain systems over the prefrontal cortex. Our digital work activities are said to trigger our reptilian, emotional brains to permanently scan for potential dan-

youngest, most important and advanced part of our brain, where more complex processes take place such as planning, representation of concepts, perception, attention, logical thinking, and moral judgements (MacLean, 1990; Siegel, 2007a \& 2007b; Siegel, 2010). 
gers, hampering cognitive functions such as concentration, working memory, and learning. Mindfulness in this repertoire is presented as a solution to prevent 'unnatural' stress resulting from these new demands in the workplace. As well-known mindfulness trainer Michael Chaskalson explains:

Mindfulness practice helps to develop emotional non-reactivity by developing those circuits of the brain that enable the lower, affectgenerating circuits to be regulated by the higher, modulating ones. It beneficially alters the connections between the (low road) subcortical limbic amygdala and the (high road) prefrontal cortex. (Chaskalson, 2011, p.107)

In addition to emotional non-reactivity, mindfulness in this repertoire promises to help "our thinking brain and our emotional brain [to] communicate more clearly to each other, so they work better together" (Tan, 2014, p. 93), allowing us to "cope with more stimuli and information" ("Mindfulness werkt voor de ondernemer," 2016). Mindfulness as brain training is geared towards increasing cognitive performance through training of the "attentional muscle" to manage "unruly emotions":

Cognitive control enables executives to pursue a goal despite distractions and setbacks. The same neural circuitry that allows such a single-minded pursuit of goals also manages unruly emotions. Good cognitive control can be seen in people who stay calm in a crisis, tame their own agitation, and recover from a debacle or defeat. (Goleman, 2013a, para. 14)

The problem of the "amygdala hijack," as Goleman famously puts it (Goleman, 1995), is that it happens automatically, and that we are unaware of it, especially when it is experienced over a long period of time. Awareness is an important part of all mindfulness programmes; awareness of your bodily sensations, of your fleeting or recurring thoughts, of storming or tucked-away emotions. In contrast to awareness of the three poisonous emotions (i.e., greed, hatred, or ignorance), as the Dalai Lama puts it, awareness in this case is directed towards the workings of our brain: Only by paying close attention to the deep influence of our brain can we change its harmful functioning. Action programmes of this repertoire are thus focused on training "at the level of physiology" (Tan, 2014, p. 28). Chade-Meng Tan, Google's in-house mindfulness advocate and founder of the popular Search Inside Yourself mindfulness programme, describes this process as follows: 
The simplest way to do it is to bring mindfulness to your body all the time. Every time you bring mindful attention to your body, you create conditions for neurological changes that allow you to become even more perceptive of your body, and consequently, of the process of emotion. (Tan, 2014, p. 76)

Thus, mindfulness is described in this repertoire as "much more than work techniques and mental strategies" because it's about "moment by moment, rewiring the neurological pathways of your brain" (Hougaard et. al., 2016, p. 165), and "rebalancing the nervous system" (Alidina \& Adams, 2014, p. 266). Generic action programmes, such as breathing techniques, small thought experiments, the labelling of emotions, and 'body scans' - standard techniques in most mindfulness programmes - are presented as solutions, precisely because they allow for neurological changes. In this repertoire, then, mindfulness is seen as a form of "neurosabotage" (Van Beek, 2012) capable of manipulating old and rigid structures in the brain.

Buddhist idiom is carefully avoided, replaced with economic valuations and metaphors. Mindfulness advocates in this repertoire describe desired neurological changes in terms of values such as productivity and efficiency, which are not only presented as promised results of mindfulness but are also described as inherent and desirable features of the changed, mindful brain itself. Authors of mindfulness books talk about mindfulness as "increasing the throughput of your brain" (Koole, 2013, p. 9), "increasing the power output of the regulation systems," and "upgrading the operating efficiency of our brains" (Tan, 2014, p. $47 \& 93$ ). Mindfulness improves the "communication between the brain's attention networks," optimizes our "limited energy supply" (Alidina \& Adams, 2014, p. $92 \& 276$ ), and strengthens the prefrontal cortex, often described as the 'CEO of the brain.' Mindfulness as brain training is a practice that "harnesses neural plasticity," which "transforms a temporary state into a more long-lasting trait of the individual" (Siegel, 2007, p. 259). The very idea of neurological change seems to become the desired end goal of mindfulness. The mindful brain in this repertoire is an efficient and productive brain.

The societal and work environment implied in this repertoire, which is detailed in Figure 6, is an environment that is highly technologized. As we have seen, major concerns deal with the rise of digital technologies (such as smartphones and email) that enable workers to be on the job 24/7. The digitization of office work is presented as something that is beyond the employee's 
control: Only our attitude towards these technologies can be changed. In order for employees and freelancers to remain productive, efficient, and effective in such an information-overflowing work environment, they have to make sure their brains are mindful and plastic enough to withstand stress, pressures, or sweeping changes. Training the brain through mindfulness, the worker is attributed with the power to overcome evolutionary-grown rigidity in the brain. Plasticity here takes the meaning of flexibility, or more specifically, resilience. Through neuroscience knowledge, mindfulness in this repertoire is presented as a necessary competence to attain cognitive performance, a new requirement for keeping up with the pace of the modern workplace and "better handle changes." Working well means being able to productively function in a technoinduced workplace - learning to be resilient enough to prevent stress and burnouts and endure changing demands.

\begin{tabular}{|c|c|c|c|}
\hline \multicolumn{4}{|c|}{ Therapeutic repertoire I: The Mindful Brain as Cognitive Enhancer } \\
\hline & \multicolumn{2}{|c|}{ SELF-KNOWLEDGE } & \multirow{2}{*}{$\begin{array}{l}\text { PRACTICAL OPTIONS } \\
\text { Action programme }\end{array}$} \\
\hline & Diagnosis & Ideals & \\
\hline SELF & $\begin{array}{l}\text { Brain overload due to } \\
\text { fight/flight response }\end{array}$ & $\begin{array}{l}\text { Productivity, } \\
\text { efficiency, } \\
\text { resilience }\end{array}$ & $\begin{array}{l}\text { Increasing cognitive } \\
\text { performance }\end{array}$ \\
\hline ENVIRONMENT & \multicolumn{2}{|c|}{$\begin{array}{l}\text { High-paced digital workplace with changing } \\
\text { demands }\end{array}$} & $\begin{array}{l}\text { Digitization of work } \\
\text { beyond individual } \\
\text { control }\end{array}$ \\
\hline
\end{tabular}

Figure 6. Therapeutic repertoire I: the mindful brain as cognitive enhancer. In this repertoire, employees are encouraged to practice mindfulness in order to increase the productivity, efficiency and resilience of their plastic brains.

\section{Therapeutic Repertoire II: the mindful brain as soft skill}

Next to a repertoire of productivity and resilience, the mindful brain is also made valuable through a repertoire of well-being. Here, actors use the mindful brain to articulate values such as compassion, kindness, empathy, and wellbeing, and tailor them to the workplace. The major concerns for employees in this repertoire are maintaining a desirable work-life balance and a lack of job satisfaction, both resulting in stress. For managers, job absenteeism, talent retention, and employees' motivation in times of economic crisis are problems for which mindfulness is seen as a solution.

The diagnosis of stress in this repertoire is similar to the first repertoire, but it emphasises a different effect of our evolutionary-grown and rigid brains. 
Here, stress is a result of the so-called negativity bias: Our brains are said to be prone towards negative information because avoiding dangers was key to our survival. As David Rock, founder of the NeuroLeadership Institute, explains in his popular mindfulness book:

As well as being a lot more anxious than happy, the limbic system fires up far more intensely when it perceives a danger compared to when it senses a reward. The arousal from a danger also comes on faster, lasts longer, and is harder to budge. . . . This also explains why upward spirals, where positive emotions beget more positive emotions, are less common than downward spirals, where negative emotions beget more negative emotions. (Rock, 2009, p. 81)

This negativity bias causes stress and makes us feel we "are losing control and that the things people do or say are threatening and negative" (Chaskalson, 2011, p. 65). To regain control over the emotional responses of the limbic system, action programmes of mindfulness in this repertoire are not geared towards increasing cognitive performance or attaining emotional non-reactivity. Instead, they are focused on practicing emotion regulation and cultivating positive emotions in order to counteract our negativity bias and to create and foster more positive experiences. Positivity is presented as "a natural mechanism that prevents our brains from functioning on too primal a level" (Hamburger \& Bergsma, 2013, p. 23).

In contrast to the generic mindfulness techniques we encountered in the previous repertoire, mindfulness as brain training here consists of action programmes such as exercises in emotion labelling, letting go, acceptance, and 'learned optimism.' This latter exercise, developed by positive psychology advocate Marin Seligman (2002), is often described as creating positive brain patterns:

Make seeking out and reflecting on what's going well in your life a simple and important part of your evening mindfulness routine. In this way, you deliberately begin to create more positive thought patterns in your brain instead of worrying-type thoughts. These patterns can even be seen in brain scans and over time become positive habits. More realistic, honest and yet positive thoughts help to engage your parasympathetic nervous system, making your brain more calm, focused and reasonable. You can then access your high-level thinking brain, which is less activated 
when overloaded with what's going wrong in your life rather than what's going well. (Alidina \& Adams, 2014, p.88)

Mindfulness here is seen as paying attention to positive things in order to "create objectivity toward our experiences" (Tan, 2014, p. 121). Thoughts and emotions that are difficult to let go should be seen as "opportunities to rewire your brain" (Hougaard et al., 2016, p. 163), and this rewiring of the brain "will help you respond mindfully to unforeseen problems that crop up in the workplace, rather than reverting to negative, unhelpful habits" (ibid, p. xiv).

To create 'more positive pathways,' meditations and thought experiments in this repertoire are directed towards cultivating values such as kindness, compassion, and empathy. Echoing the research of Davidson and others, these values are described as attainable qualities of the brain: happiness, kindness, compassion, and empathy "come preinstalled in our brains; we are all hardwired to be empathic" (Tan, 2014, p. 145). We only have to unlock our potential to access these states by practicing mindfulness as brain training:

We can train and develop the mind to create inner peace, happiness, and compassion. The best part of this training is that we do not even have to force ourselves to have those qualities; they are all naturally already within each of us, and all we need to do is create the conditions for them to emerge, grow, and flourish. We create those conditions through meditation. (Tan, 2014, p. 173)

Kindness becomes described as "the direct neurological opposite to unpleasant states of mind" (Hougaard et al., 2016, p. 136), and by practicing kindness it can become "our default neurological reaction" (Hougaard et al., 2016, p. 137). The research of Davidson and his colleagues is frequently mobilized in this repertoire as evidence that meditation increases feelings of optimism and happiness. It is argued that mindfulness activates the "left part of the brain," which is good since "at the left, brains are more happy and optimistic than at the right side," as we read in popular mindfulness books for managers (Van der Roest, 2013, p. 87). Plasticity here is used to justify the claim that 'real' self-transformation is possible and that happiness, compassion, and kindness are within our reach, as trainable brain traits.

In the previous repertoire the focus of attention was only inwards. Here, attention control is also focused on others. Cultivating compassion, kindness, and empathy through mindfulness is seen as beneficial for communal life at the office. Managers and leaders are specifically addressed. Empathy and kindness, 
for example, improve managerial qualities. Kindness has the possibility to "create trust and understanding" by which managers become more able to "skilfully negotiate and manage their [employees'] concerns" (Tan, 2014, p. 128). Activating the "left part of the brain is linked to an increase in optimism, satisfaction, the meaning of life, and positive leadership qualities" (Dewulf, 2014, p. 130). For example, compassion and honesty are often seen as beneficial for teamwork as they would encourage mutual support, a better work climate, and increase output (Koole, 2013, p. 105).

Similarly, happiness and well-being are associated with job satisfaction and are presented as prerequisites for workplace values such as creativity and innovation. As Chaskalson explains:

The idea that innovation and creativity are associated with happiness and well-being sounds intuitively obvious. Thanks to Davidson and others, however, we now know something of neuroscience that underpins that observation - and we might therefore be more readily able to train for these factors. (Chaskalson, 2011, p. 78)

Well-being is thus not only good in itself but is also seen as beneficial for creativity and innovation. Positive emotions "widen our attention and our receptiveness to the new and unexpected" (Goleman, 2013a, p. 55) and allow for creativity and 'flow.' Well-being also strengthens the company culture as employees become more motivated, engaged, and "are less inclined to search for other jobs [because] they value their co-workers more, and they feel in general more satisfied and happier at work" (Dewulf, 2014, p. 133). In contrast to the Dalai Lama's eudaimonic view of happiness as a "wholesome, fulfilling life, in harmony with one's self and surroundings" (Dalai Lama, 2005), happiness and well-being in this repertoire become defined hedonically as the absence of negative affect and the affirmation of positive affect.

In what kind of work environment are compassion, creativity, well-being, and a positive company culture needed? The implied societal and work environment in this repertoire is one that puts quite a bit of pressure on employees and the company culture. The work environment is presented as precarious and characterized by permanent change (in projects, project teams, and demands). In such an environment, it becomes beneficial for employees and managers alike to be able to maintain creativity, collegiality, job satisfaction, and an inviting company culture. Instead of necessary competence to remain productive, as in the first repertoire, mindfulness here becomes a soft skill, a social attitude that 
improves working life both for the individual and the company culture. Working well in this repertoire means being positive, creative, kind, compassionate, and empathic. Values such as kindness, compassion, and well-being are however not only seen as ends-in-themselves but are tailored to benefit the workplace and aimed at preventing stress and improving creativity and communal life at the workplace. While the dynamic and changing character of contemporary work seems to be beyond the control of the employee, the proximate environment - the company culture - can be changed for the better.

In contrast to the first repertoire, Buddhist idiom, values, and poetic rhetoric are abundant here - though not always explicitly labelled as such. While advocates thereby re-ethicize mindfulness in this repertoire - using the plastic, mindful brain to articulate values and an action programme similar to traditional Buddhist mindfulness meditation - the justifications and valuations for cultivating compassion, kindness or empathy differ: They are not sought by referring to their balancing effect against the "poisonous diseases" of hatred or greed but rather are desired as valuable because of their lasting effect on the plastic, mindful brain. Plasticity in this repertoire, shown in Figure 7, takes on the meaning of self-transformation.

Therapeutic repertoire II: The Mindful Brain as Soft Skill

\begin{tabular}{lllll}
\hline & \multicolumn{2}{l}{ SELF-KNOWLEDGE } & & PRACTICAL OPTIONS \\
\cline { 2 - 3 } SELF & Diagnosis & Ideals & Action programme \\
& $\begin{array}{l}\text { Stress and unhappiness } \\
\text { due to negativity bias }\end{array}$ & $\begin{array}{l}\text { Compassion, } \\
\text { kindness, creativity, } \\
\text { well-being }\end{array}$ & $\begin{array}{l}\text { Emotion regulation and } \\
\text { cultivating positive } \\
\text { emotions }\end{array}$ \\
& $\begin{array}{l}\text { Dynamic, precarious economy, resulting in high- } \\
\text { pressure company culture }\end{array}$ & $\begin{array}{l}\text { Company culture can be } \\
\text { changed }\end{array}$ \\
\hline
\end{tabular}

Figure 7. Therapeutic repertoire II: the mindful brain as soft skill. In this repertoire, employees are encouraged to practice mindfulness to become more compassionate, kind, creative and happy in the workplace.

\section{Wholesome Working in a Technologized Work Culture}

Think of a world in which improving performance goes hand in hand with increasing kindness. And a world in which kindness is valued as much as efficiency and effectiveness, as much as revenue per share or operating cash flow. (Hougaard et al., 2015, p. xii) 
The two therapeutic repertoires of the mindful brain in the context of work help to show how mindfulness has gained ground in the business realm. Using a neuroscience understanding of mindfulness, advocates of mindfulness in the first repertoire are able to address concerns of productivity in a digitalized workplace, aligning mindfulness to a register of individual, economic values. In the second repertoire, a neuroscience understanding of mindfulness is used to address concerns of well-being and communal life at the workplace, using the mindful brain to introduce social values such as compassion, kindness, and empathy - values that are not traditionally seen as part of the office world - into the context of work. Productivity and efficiency are hardly considered values at all in the context of work; they remain implicit as non-controversial elements of working life. Values in the second repertoire, such as compassion, empathy, and well-being, are recognized as such by mindfulness advocates, since their presence in this context is less self-evident. Through the mindful brain, actors are able to put these values forward in an organizational setting, using the hard means of neuroscience as justification.

A therapeutic repertoire is an analytical concept: in practice, both mindful brains and ideals of working well are used side by side. The popularity of the mindful brain in the context of work lies precisely in this combination. Talking about productivity alone would probably not have resulted in such a widespread adoption of mindfulness by employees and managers alike. By combining a language of control and productivity with the language of empowerment and well-being, the mindful brain is celebrated as a solution to concerns of both employees and managers. The two repertoires presented here thereby reinforce and complement each other, although they are geared towards different endsin-view. Both are needed to introduce mindfulness on the work floor. It is the susceptibility of the plastic, mindful brain which affords actors to re-ethicize a neuroscientific understanding of mindfulness in different moral discourses of working well.

It took quite some effort for mindfulness to travel from its Buddhist tradition to the office floor. Having traced the trajectory of valuations of the mindful brain in the context of work, the question now rises to what extent the mindful brain actually challenges ideals or practices of work. Does knowledge of the mindful brain shed a new or different light on our perception of the stressed employee and the ideal employee? Does it afford new action programmes for managers and employees to deal with stress and to work well? And in what kind of societal and organizational environment does mindfulness emerge as a 
cognitive enhancer and soft skill? If we compare the mindful brain as sciencebased management intervention to contemporary and past valuations of work, some moral changes become visible.

\section{Knowing Your Feelings at Work}

To what extent does the mindful brain lead to a new way of looking at the contemporary employee and manager? Whether mindfulness can lead to new personal insights in practice is not the topic here; rather, it is about the kind of selfknowledge of employees and managers that the mindful brain at the workplace proposes. In the repertoires of the mindful brain at work, self-knowledge takes a particular form, namely, knowing how your brain works at work, especially regarding emotions.

In both repertoire, stress can be countered by knowing and managing your feelings in the workplace. In the first repertoire, employees' emotions have to be regulated in order to increase cognitive performance, whereas in the second repertoire, unruly emotions should be replaced by a cultivation of more positive and socially engaged emotions, such as kindness, compassion, and empathy. Through mindfulness, we thus come to know the contemporary employee as a deeply emotional being.

Explaining the diagnosis of stress through a lack of emotion regulation has the combined effects of normalizing stress and individualizing it. In the repertoires of a mindful brain, stress is not primarily seen as a sign of too much work, but as a mismatch between our underdeveloped brains and our $21^{\text {st }}$ century workplace. As such, every employee in the contemporary workplace is presented as biologically prone to stress, turning stress from a specific problem to a widely shared yet individualized, biological one. By framing stress as a problem of brain physiology, mindfulness advocates are able to take away the burden of responsibility for non-neurological causes of stress. Stress thus becomes a problem which an employee can best deal with through meditation. Through the use of the plastic, mindful brain, mindfulness advocates are able to cast the employee as being naturally stressed, yet able to overcome this inclination at the same time. The focus thereby shifts towards the psychological and social life of the employee.

The focus on the emotional life of the employee reflects a development in contemporary management that began in the 1990s. The psychological and emotional dimensions of the employee are seen as characteristic of new types of work that emerged in the information economy or knowledge society. In the 
1990s, employees increasingly had to learn to cooperate in short-term project teams (cf. Boltanski \& Chiapello, 2005; Sennett, 1999). In high-pressured, digitally distracting, open bullpen offices, the ability to remain creative and work collegially emerge as additional skills. The focus on the emotional life of employees and managers in mindfulness discourse thus does not challenge our understanding of what an employee and manager is or should be, but rather reflects these ongoing moral changes in Western working culture.

In the 1990s, the possibility for personal fulfilment was often seen as the hallmark of good work. Contemporary work, as idealized through the therapeutic discourse of mindfulness, seems rather a vocation: work as a disciplined lifestyle that flourishes with professional and personal fulfilment and happiness. The ideal employee that emerges through the repertoires of the mindful brain is an emotionally controlled employee who is cognitively productive and efficient and contributes to a satisfying company culture by being happy, compassionate, kind, and empathic to co-workers. Robbed of his characteristic robes, left with brain training as a bare, yet mouldable form of ascesis, the monk in the office appears to combine individual emotional self-control with social self-fashioning.

As cultural sociologist Illouz (2008) has convincingly shown, emotional control in the context of work requires the capacity of the individual employee and manager to regulate emotions and to maintain oneself socially by communicating about these emotions to others in a particular manner (p. 103). As Illouz argues, emotional control in contemporary work implies a disengaged self that is characterized by self-control, such as through the kind of emotion regulation one may achieve by improving one's cognitive performance in the first repertoire. At the same time, emotional control implies a sociable self, in which emotions should be suspended, according to Illouz (2008). Emotions such as anger, contempt, or shame (which are feelings about social relationships) would disrupt or hamper the "expected smoothness of social interactions" that contemporary forms of work necessitate (Illouz, 2008, p. 104). While such 'negative' feelings indeed seem not welcome at the mindful workplace, not all emotions are suspended in the case of the mindful brain. In the second repertoire, we saw that employees and managers are encouraged to cultivate positive emotions and values (such as compassion, kindness, and empathy), precisely because these would strengthen communal (and communicative) life at the office, as well as foster individual creativity and well-being. In other words, through the mindful brain, a disengaged self and a sociable self are made to reinforce each other. 


\section{Practising for Performance and Well-Being: mindfulness as neuroascesis}

The way we understand ourselves as employees has consequences for what we think we can do in practice. To what extent does the mindful brain challenge or manage the ways employees and managers can work well? In both repertoires, the employee and manager are cast as individuals in need of emotional selfcontrol: in the first repertoire in order to guard against digital distractions and maintain productivity, and in the second repertoire to improve creativity and job satisfaction while fostering a healthy company culture. The need to increase workers' self-control is a recurring theme in management history. But where the lazy and disobedient factory workers were attributed by their "labour aristocrats" a lack of moral character, here the lack of self-control of employees is attributed to their rigid brain circuits and a lack of brain plasticity - which employees can readily train through mindfulness. Skills that need to be developed through training are attention control and cognitive performance in the first repertoire, and compassion, kindness, empathy, and well-being in the second.

Mindfulness in both repertoires is presented as a form of brain training that can be compared to going to the gym. This idea of working directly on the brain is a perfect example of what we can call neuroascesis (Ortega, 2011). Neuroascesis can be described as "brain fitness" - practices and prescriptions of cerebral self-help (Ortega, 2011). Despite the focus on the body in mindfulness techniques, the real target of mindfulness seems to be the plastic, changing brain. Only by learning how to use our mind in a specific way, by paying attention, can we put the primitive brain back in its place. The effects of changing your brain through techniques of mindfulness are seen as of a higher order than changes in thought or behaviour alone. It is implicit in all action programmes that a change in brain structure is more solid, or more fixed, than a changed mind or changed behaviour. It seems that through training the plastic brain, we exchange a bad form of brain determinism (the "monkey brain" or amygdala hijack) for a good form of determinism (a mindful brain). Mindfulness advocates thus combine a story of empowerment (overcoming the primitive brain) with the promise of structural or grounded change (self-transformation).

Meditation and the development of skills such as empathy and kindness seem a relatively new intervention on the work floor. However, the form this meditation takes - as brain training - is not revolutionary. Practices of neuroascesis can be traced back to the times of phrenology, when people were urged to increase the strength and energy of different brain parts. As Ortega (2011) and 
Cooter (1984) show, phrenological prescriptions of exercising the brain reflected Victorian virtues of sobriety, moderation, chastity, and self-improvement (Ortega, 2011). While mindfulness does not challenge this means of enhancing oneself, the virtues that we now ascribe to an exercised or plastic, mindful brain are obviously quite different. The ideal employee seems to embody different desirable virtues: A mindful employee is focused and flexible, compassionate and competitive, resilient and authentic, compliant and innovative - all at the same time. The power of the mindful brain lies precisely in the promise that we don't have to make trade-offs; we can have it all. We can be relaxed and resilient, happy and high performing (cf. Cederström \& Spicer, 2015; Davies, 2015). As many of these virtues are projected on the plastic brain - such as efficiency, productivity, resilience, and the potential for self-transformation and well-being - plasticity itself seems to become a virtue that characterizes a good employee.

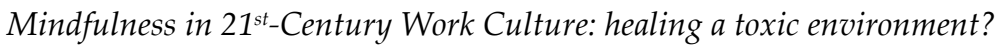

In what kind of societal and work environment does this ideal employee flourish? What does the emergence of the mindful brain imply about the amount of control we think we have over our work culture? Strikingly, while mindfulness advocates articulate concerns about stress and anxiety due to new demands in the workplace - digital technologies, high workloads, economic crises - a more explicit evaluative reflection on the changing work environment in our contemporary society is often absent in mindfulness programmes and books. Issues related to work itself - how our digitalized knowledge society affects different economic sectors, different levels of employment, or different skills or tasks are rarely critically reflected upon in mindfulness programmes. In the rare instances when mindfulness programmes do address such issues in a critical manner, it remains the individual who is attributed the agency and capability to change both her own performance, and the company culture in general. Work itself seems to a great extent black boxed as an autonomous force outside of our scope of influence.

This lack of attention towards the meaning of contemporary work and working conditions in mindfulness programmes has raised scorn from critics of mindfulness. The Dalai Lama spoke at the Society for Neuroscience of the ways in which humans suffer - due to destructive emotions of greed, ill will, and ignorance - and how a combined effort of neuroscience and Buddhism could have "far-reaching implications for society at large" (Dalai Lama, 2005). Greed, ill will, and ignorance are hardly addressed by mindfulness advocates. This led 
some (Buddhist) critics to argue that mindfulness in the workplace, dubbed by them 'McMindfulness,' neglects the "unwholesome roots of greed, ill will and delusion"; they fear that mindfulness at the workplace reinforces those roots rather than "awakens individuals and organizations" to them (Purser \& Loy, 2013). ${ }^{48}$ These authors go against the value work of de-ethicizing mindfulness by Davidson and others and try to reinstate the Buddhist and ethical context of mindfulness, as they see a decoupled and decontextualized form of mindfulness as a danger that only strengthens the status-quo of greed and profit-overpeople.

At first sight, the therapeutic discourse of the mindful brain in practice seems to underscore this critique. It is not difficult to imagine how mindfulness and happiness at the workplace could service managers and companies as yet another instrument with which to control their personnel and raise modern work expectations - when happiness or kindness turns from trainable skill to required competence. Managers can indeed offer mindfulness courses as management intervention to channel discontent at work: for example, offering mindfulness to prepare workers for a reorganization. This is a genuine danger of the widespread adoption of mindfulness. A danger that depends on the extent the propagation of mindfulness comes with other options to deal with stress, for example through workplace measures, institutional policies, or political decision-making.

But the emergence of the two different repertoires also brings a nuance to such critiques. For the claim that mindfulness further individualizes the problem of stress, we can find convincing examples in both repertoires. By framing stress as a physiological problem of a rigid brain and addressing it by encouraging workers to improve their cognitive performance, creativity, and well-being, mindfulness practices do seem to deal with stress solely in individualistic terms. At the same time, however, by interpreting plasticity as selftransformation, mindfulness could also be valued as a way to address communal life at the office and to introduce social-oriented values such as kindness, compassion, and empathy into the workplace (as we saw in the second repertoire). Mobilizing mindfulness to address the concerns of a high-paced digital workplace with changing demands, and a high-pressured and dehumanized company culture, can put these issues on the broader (organizational or politi-

\footnotetext{
${ }^{48}$ Similarly, other authors warn for possible side-effects, such as the avoidance risk, when mindfulness is used as a form of sensory retreat instead of critical, self-reflective thought (see for example Brendel, 2015; Lindahl, Fisher, Cooper, Rosen, \& Britton, 2017).
} 
cal) agenda. By cultivating compassion, empathy, and kindness at the workplace, not only managers but also employees receive a legitimized discourse to address otherwise taboo work issues (such as stress) that may form obstacles to achieving job satisfaction. Even though well-being at the workplace seems far off from the Dalai Lama's eudaimonic view as a "wholesome, fulfilling life, in harmony with one's self and surroundings" (Dalai Lama, 2005), one could argue that mindfulness does function as a gateway that can make employees' (social or personal) grievances acceptable for conversation. A focus on the plastic brain appears not necessarily purely individualistic; through the mindful brain, the environment is addressed through the individual.

Though the second repertoire seems to problematize critiques of mindfulness as being purely individualistic, it fails to convey a substantial critique. Values such as compassion, empathy, and kindness have the potential to be critically used. For example, they could be used to argue the need for reducing long working hours, lowering the work pace, or protecting the private sphere from the invasion of work. One could imagine a way to mobilize compassion, the diagnosis of the "monkey brain," or the vulnerability of a plastic brain (instead of only its potential to change) to argue for more time off, a reduction in overtime, a company ban on reading and sending work-email in the evenings or holidays, or even the introduction of a basic income and shorter working weeks. While the plastic brain as argument in principle would allow such valuations - i.e., by attributing also some agency to the environment, which imprints the brains of employees - such suggestions are seldom made in corporate mindfulness programmes, if at all.

In contrast to earlier pleas for empowerment of the worker, mindfulness seems to evoke a moral change. While empowerment usually came with a fullfledged critique on, or a resistance towards, the capitalist system, the form of empowerment that is mobilized through mindfulness is aimed at encouraging the individual employee and manager through mindfulness to cope with, and thereby adapt to the demands of the system. As such, the mindful brain appears to be a fitting example of what Boltanski \& Chiapello (2005) have coined the 'new spirit of capitalism.' This new spirit of capitalism incorporates previous critiques against capitalism as dehumanizing and inauthentic and takes its ideal of the manager and employee from the artist: celebrating creativity, authenticity, self-expression, and flexibility (Boltanski \& Chiapello, 2005). As Cederström \& Spicer (2015) have argued, the underlying assumption behind mindfulness programmes at work is the claim that "impermanence is an immediate reflec- 
tion of the basic nature of reality" (Cederström \& Spicer, 2015, p. 25). As permanent change itself is presented as the only constant individuals in contemporary working culture can expect, a constant that is presented as beyond our individual control, the best thing an employee can do to harmonize herself and her proximate company culture to such an environment is to embody this change by cultivating a plastic brain.

Whether mindfulness is an adequate solution to the problems of our contemporary work culture, where "average actual working time has become more intense, faster paced, more flexible and longer" (Ehmer \& Lis, 2016, p. 1), becomes thereby doubtful. Mindfulness as coping mechanism for the high-paced and precarious character of contemporary work could well be suited for the job of dealing individually with digital distractions, and socially with a stressful company culture - but this is not for me to judge but for the individual mindfulness practitioner. However, one does have to acknowledge that since substantial critiques on the conditions of work are not sufficiently addressed in mindfulness programmes, the mindful brain can only be half of the solution.

\section{Conclusion}

In this chapter, I have explored how neuroscience helped mindfulness travel to the workplace. A neuroscience-based understanding of mindfulness indeed has made mindfulness accessible to a wider audience, as its enthusiasts and critics both agree (cf. Cederström \& Spicer, 2015; Davies, 2015; Purser, 2016; Tresch, 2011). However, as this chapter has shown, this was not an automatic process; it was through deliberate strategies of de-ethiczation and re-ethicization that mindfulness became seen as acceptable and desirable in the workplace. The mindful brain gained traction in the context of work precisely because it could be reethicized in multiple ways, aligning to economic ideals of increasing cognitive performance and efficiency as well as to ideals of achieving personal well-being and a satisfying and inviting company culture. The popularity of the mindful brain therefore lies not solely in its scientific credibility and authority but rather in its promise to fulfil the conflicting demands of our highly technologized knowledge economy, both for employees and managers.

Through the mindful brain, we come to know the contemporary employee as a deeply emotional being. This focus on the emotional life of the employee does not challenge how our understanding of what an employee and manager 
is or should be but rather reflects developments in management theories and Western working culture since the 1990s. Work in mindfulness programmes is treated as a kind of vocation, in which flourishing depends on a disciplined lifestyle. Self-discipline becomes a combination of emotional self-control (a disengaged self) and social self-fashioning (a sociable self), where the regulation of emotions involves increasing cognitive performance and the cultivation of social values such as kindness and empathy to benefit the organizational culture. In mindfulness programmes, a disengaged self and a sociable self are made to reinforce each other.

While meditation can be seen as a relatively new intervention on the work floor, the form it takes as brain training, or neuroascesis, is not. Compared to older forms of brain training, we do see the articulation of different virtues that are characteristic of contemporary working life: a modern, mindful employee is focused and flexible, compassionate and competitive, resilient and efficient, creative and innovative - all at the same time. The power of the mindful brain lies precisely in the promise that we don't have to make trade-offs between different contemporary values and virtues of working well; we can have it all.

The mindful brain in the context of work thus did not result in substantial moral changes on the level of employees' self-understanding or the practical options they have for becoming a good worker. Instead, moral change only takes the form of some minor shifts in the relationship between ideals: Mindfulness as individual empowerment does not rule out addressing and improving the social environment at work. While the mindful brain in practice thereby seems to bring a nuance to critiques of individualism, the lack of substantial reflection in mindfulness programmes on modern working conditions does result in the danger that mindfulness strengthens the status quo rather than challenges it. When the world of work is seen as an autonomous force of continuous change, and change itself is heralded as desirable, the plastic brain of a mindful employee seems to become an important virtue at work.

Whereas the plastic brain of the adolescent in the previous chapter was presented as a given, the plastic brain of the employee in the context of work becomes a task and an ideal. Values that were deemed desirable in the plastic adolescent brain (in the second repertoire) returned in the adult, working phase of life as virtues that need to be practiced if they are to be maintained: selfregulation, flexibility, resilience, creativity, and sociality. In contrast to the previous chapter, where agency for changing the brain was given to the environ- 
ment, the agency for changing the brain of the employee is given to the individual subject.

When our teenage children's brains have finally grown and long left the building, and we have managed to say goodbye to our mindful days at the office, we enter a different stage in life. Whether you call it the autumn years or the golden age, there is one thing that seems to remain stable throughout our lives: our plastic, changing brains. Just as the teenage brain and the adult brain, the ageing brain is presented as plastic: as being prone and subject to ongoing changes. What would happen to a plastic brain that no longer has to learn to be an adult, a brain that no longer has to productively and socially perform at the office? How would plasticity be valued in the context of ageing? In the next chapter, we explore what it means to age well with a plastic brain. 



\section{CHAPTER 4}

\section{Engaging the Ageing Brain: cognitive decline and ageing well}

\section{Introduction}

very morning around half past ten, around 30,000 seniors in the Netherlands
tune in to Omroep MAX, a Dutch TV broadcaster for seniors ("TV Kijkcijfers," 2016)..$^{49}$ In the 15-min show Max Geheugentrainer, a contestant in the studio (together with the viewers at home) is presented with different types of puzzles and memory exercises ("What was the colour of the clown's hat in the previous video?" or "Which groceries were on the shopping list?"). Most of the participants in the studio manage to answer the questions correctly, thereby winning a game console. The TV programme, running daily since 2007, aims to give their viewers their "daily portion of brain gymnastics" (Max Geheugentrainer, 2016).

MAX Geheugentrainer is based on Dr. Kawashima's BrainAge: Train your Brain in Minutes a Day! (2006), a popular brain game for the handheld game console Nintendo DS. Since its introduction in 2006, Nintendo has sold more than 34 million copies of BrainAge and its successor, BrainAge 2 (Nintendo, 2016). Some ten years later, brain games for the elderly have gone mainstream and become a booming industry. Companies such as Lumos Labs, Cogmed, CogniFit, and

\footnotetext{
${ }^{49}$ In this chapter, I use the relatively neutral terms "senior" and "elderly" to describe people of older age.
} 
Posit Science successfully sell computer-based cognitive training software, website memberships, and mobile apps with monthly subscription fees, all targeted to senior consumers. Advertised as being based on the most recent insights from the neurosciences, these commercial successes promise to improve cognitive functioning, delay age-related cognitive decline, and even stave off Alzheimer's. Seniors en masse subscribe and use such cognitive training software on a daily basis: Lumosity for example claims they have 70 million active users worldwide. In the Netherlands, more than 200,000 people use the brain games on the website Neurocampus.com. Brain games (such as those of CogniFit) are not only used by consumers; they are increasingly finding their way into nursing homes and clinical practices. According to market research firm SharpBrain, the global market of brain training applications surpassed \$1 billion in 2013 and is estimated to reach $\$ 6$ billion by 2020, of which approximately $\$ 3.38$ billion is reserved for cognitive assessment and training software (SharpBrains, 2013 \& 2015).

Perhaps not surprisingly, the popularity of these commercial applications of neuroscience knowledge sparked a worldwide scholarly and public controversy. In 2012, the Stanford Centre of Longevity together with the Max Planck Institute for Human Development issued a 'consensus letter' signed by some 70 scholars (cognitive psychologists and neuroscientists studying cognitive aging), warning consumers about the inflated promises and lack of scientific validity of the brain game industry ("A Consensus on the Brain Training Industry," 2014). In response, a group of 133 neuroscientists rebutted the letter, claiming that the critical scientists had ignored the potential of the plastic, ageing brain (Koenig, 2014). The quarrel between these groups of scholars was widely portrayed in public discourse in the Netherlands, with headlines such as "Digital Brain Training is a Waste of Time" (Steenhorst, 2014) and "Brain training scientists think that people worry too much" (Van Hintum, 2009)

Brain games are an application of an emergent understanding of the ageing brain as particularly plastic. The once dominant idea that the brain passively deteriorates when getting older, losing neurons along the way, is replaced by a view of an actively adapting brain, still capable of neurogenesis and change. The ageing brain has "a remarkable built-in ability to strengthen and grow the person that you are, at any age," as eminent neuroscientist, plasticity pioneer, and cofounder of Posit Science Michael Merzenich puts it (2013, p. 2). This newly found plasticity of the ageing brain invites its appropriators to articulate suggestions for seniors regarding what they should do with their ageing brains. The ageing brain allows neuroscientists and others to address the question that 
plagued philosophers for centuries, a question that forces itself upon all of us when the time comes: What is it to age, and how can we do it well?

In this chapter, I explore how a neuroscience understanding of ageing is used to address these timeless ethical questions. How is the ageing brain used to articulate what seniors should do or not do? How does one age well with a plastic brain? And how is a neuroscience understanding of ageing made to challenge what it means to age well? Instead of following a specific actor or a group of actors like I did in the previous two chapters, my entry point here is the controversy around brain games for the elderly. ${ }^{50}$ It is through the advent of brain games, and the widely reported controversy they invoked, that a neuroscience understanding of ageing became popularized in (Dutch) public discourse. I explicate how brain game publishers make their products valuable for seniors and how those valuation efforts became subject to critique. I then trace valuations of ageing brains in popular science and advice literature. As we will see, it appears that both brain games and the controversy they evoked function as an ethical yardstick in relation to which ageing-brain advocates can position themselves as they counter-propose different diagnoses, courses of action, and views on ageing. I will again present two therapeutic repertoires in which knowledge of the plastic, ageing brain is mobilized by neuroscientists, gerontologists, science journalists, and others to articulate what it means to age well, what seniors could or should do to achieve that goal, and why.

In the context of a historical demographic shift reflecting a 'double greying of society' - one in which we increasingly live longer, but we also live longer with chronic diseases - the meaning of growing older and the role of seniors in society have become topical issues. It is within this context that a neuroscience

\footnotetext{
${ }^{50}$ My empirical body of sources for this case is limited by focusing on the uptake of the ageing brain in Dutch public discourse on ageing, including the controversy surrounding brain games. My empirical material includes popular brain games specifically tailored to seniors (BrainAge, BRAINHQ, Cognifit, Lumosity, Neurocampus.com, and HAPPYneuron), their advertisements, and their websites. I also conducted a LexisNexis search on "ouderen AND brein OR hersenen," resulting in 2,649 articles from Dutch newspapers and magazines from the period 2000 through 2015. I excluded duplicates, articles that only dealt with clinical diagnoses, did not address seniors in a prescriptive manner or did not discuss ageing, or only marginally addressed knowledge of the (plastic) ageing brain. This resulted in a sample of 319 relevant articles. My empirical material further included popular Dutch (translated) science books and self-help books on the ageing brain, such as Goldberg's De Wijsheidsparadox [The Wisdom Paradox] (2006), De Lange's Breinbewust Leven (2012), Van der Zee's Het Vitale Brein (2012), Aleman's Het Seniorenbrein [Our Ageing Brain] (2014), Merzenich's Soft-Wired (2010), Sitskoorn's Lang Leven de Hersenen (2008), and Cozolino's The Healthy Aging Brain (2008). Taken together, this body of sources encompasses the rise of a neuroscientific understanding of ageing in the public sphere in the Netherlands.
} 
understanding of ageing has emerged. To explore whether the ageing brain challenges ideas of ageing and how seniors can or should care for themselves, I first give a short sketch of how the meaning of growing old, and ideas of what seniors could hope for, changed throughout history - focusing on the Netherlands in the $20^{\text {th }}$ century.

As we will see, the plastic ageing brain comes with similar valuations as plasticity in the previous cases. While the agency for change is initially located in the ageing brain itself (instead of attributed to the individual subject as in the case of the mindful brain, or the environment in the case of the teenage brain), seniors are not presented as having to undergo these brain changes passively. Ageing is no longer an automatic process, and to do it well requires the active engagement of seniors - engagement in activities that often bear a strong resemblance to the brain training activities we encountered in the mindful brain case. At the same time, like the teenage brain, the plastic, ageing brain is seen as a distinctive type of brain with its own unique characteristics. Catering to a variety of concerns, the therapeutic repertoires of a plastic, ageing brain appear to present a powerful and seductive view of ageing well, where hope is combined with consolation, and losses are overcome or turned into individual or societal gains.

\section{Ageing Well Throughout History}

Aging was for a long time hardly considered a topic for scientific inquiry. Likewise, in the history of philosophy, growing old was for many early philosophers not a subject of contemplation - their ponderances favoured death and mortality, which they were far more likely to face than growing old. Exemplary of this neglectful view are the famous descriptions of older people by Aristotle. In Rhetoric (II12\&13), he describes the character of elderly men as hesitant, cynical, distrustful, suspicious, small-minded, shameless, cowardly, querulous, with a chilly temperament, and inspired by the love of gain (Aristotle, Rhetoric, III2\&13). We find a less negative description in the first philosophical essay dedicated to old age by the Roman Cicero. In contrast to Aristotle, Cicero celebrates the benefits of old age in Cato Maior de Senectute (On Old Age, 44 B.C.), such as prudence, worthy life experience, wisdom, and a lack of desires. For Cicero, if old men are "morose, troubled, fretful, and hard to please," then it's not caused by old age as such but by a "faulty character"; senility then is not characteristic of all old men, but only "of those who are weak in mind and 
will." While for Cicero old age comes with intrinsic goods, one has to cultivate and strengthen one's body and character to "garner Nature's fruits":

It is our duty . . . to resist old age; to compensate for its defects by a watchful care; to fight against it as we would fight against disease; to adopt a regimen of health; to practice moderate exercise; and to take just enough of food and drink to restore our strength and not to overburden it. Nor, indeed, are we to give our attention solely to the body; much greater care is due to the mind and soul; for they, too, like lamps, grow dim with time, unless we keep them supplied with oil. (Cicero, 44BC)

Where Aristotle only saw frailty, evanescence, decline, and a loss of character, Cicero sees wisdom, prudence, and an exemplary function for old men in Roman society based on their life experience, provided that men fight old age as disease. Or "senectus morbidus est," as Seneca would put it. ${ }^{51}$ This ambiguity of old age, characterized by both problems and intrinsic goods, runs as a red line throughout the history of thinking about old age - what comes to count as 'good' or 'bad' in ageing, and which part gets the upper hand, shifts from time to time.

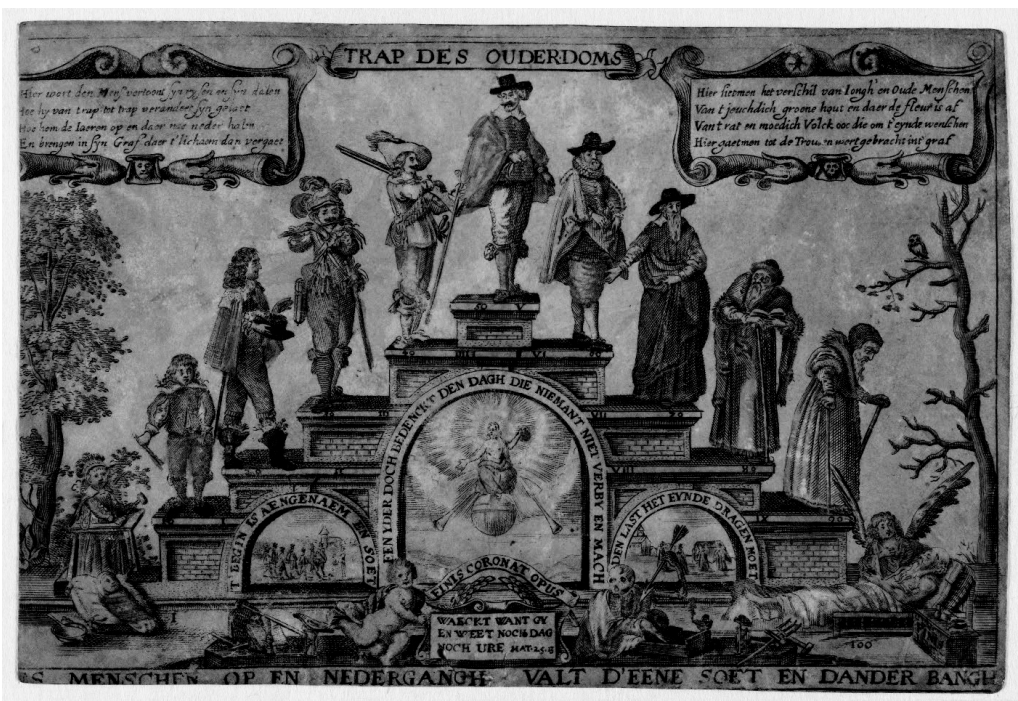

Figure 8: Trap des Ouderdoms, anoniem, 1640 - 1660. Source: Rijksmuseum.

${ }^{51}$ Perhaps needless to say, these philosophers did not talk about slaves, women, or other classes they deemed 'lower.' 
Roughly sketching views on old age from medieval times towards modernity, one could say it is best captured in the adagio memento mori. ${ }^{52}$ Death due to accident or disease before reaching the age of 40 was common. Due to a low life expectancy and high mortality rates, good ageing in medieval times was associated with public withdrawal and religious introspection - symbolized with vanitas in many $17^{\text {th }}$ century paintings, or the gravures that depict stages of life such as Trap des Ouderdoms (Cole, 1992, p. 12; De Lange, 2013). Philosophers such as Petrarca, Montaigne, Rousseau, Goethe, and Schopenhauer devoted essays to old age. In his essay De l'âge (1580), for example, Montaigne recognizes that while knowledge and experience may increase over the years for those "who make the best use of their time," he disliked his own old age, which he saw was characterized by a decay of "vivacity, promptitude and steadiness" (Montaigne, 1580). Supposedly suffering from kidney stones, Montaigne repeats the words of Lucretius, who cried: "When the body is shaken by the blows of time, and the members have lost their vigour, the spirit grows weaker, the mind slumbers, and the tongue babbles" (Montaigne, 1580, as cited in Friswell, 1976). ${ }^{53}$

It was only in the $19^{\text {th }}$ and $20^{\text {th }}$ centuries that old age became more common and thus became a subject of more extensive scrutiny and study. For Victorians, old age was characterized by mental perturbations, insanity, and dying (Millington, 2012). While old age for a long time meant becoming poor and isolated, a combination of developments slowly helped to improve the social and economic status of seniors. The increased health standards and longevity, the enactment of social security laws for seniors (such as the 1913 Ouderdomswet and the 1957 Algemene Ouderdomswet in the Netherlands, which gave seniors some basic income), the emergence of hospices and homes for the elderly, and the emergence of the scientific discipline of gerontology all helped to establish old age as a distinct (biological) stage of life, social category, and object of care (cf. Bijsterveld, 1996). In the 20th century, ageing turned from religious mystery to object of scientific management (Cole, 1992).

\footnotetext{
52 Just as in the previous chapters, the historical overview I sketch is necessarily limited. I focus on the history of ideas of ageing (well), with an emphasis on the $20^{\text {th }}$ century, when ageing became an object of scientific management (Cole, 1992). For reasons of clarity and brevity, I do not include different histories for different social or economic classes, nor do I differentiate between gender. The goal is to sketch broad historical changes in our ideas and ideals of ageing.

${ }^{53}$ See the excellent collection of historical philosophical texts on the art of ageing well: Dohmen \& Baars (2010).
} 
After WWII, old age became increasingly perceived as a social and political issue. For example, in La Vieillesse (1970), Simone de Beauvoir - after Cicero the first philosopher who wrote a comprehensive work on old age - criticized the cultural taboo on old age, questioned whether the deplorable condition and isolation of old people (and especially women) is inevitable, and wondered how society should take responsibility for improving the lives of its seniors (Dohmen \& Baars, 2010, p. 337). During the better part of the 20th century, aging itself was mostly seen as a period of life characterized by decline, loss, dependence, and frailty. The economic status of seniors slowly improved, fuelled in the Netherlands by the rise of elderly homes in the 1960s - first an ideal for the lucky few, but later on also accessible to working-class seniors. The idea of ageing well changed. Good ageing in the context of the Dutch welfare state in the second part of the $20^{\text {th }}$ century can be described through the phrase dolce far niente: enjoying sweet retirement and not having to work (De Lange, 2013).

Within the diverse field of gerontology, the idea of ageing as solely characterized by decline and loss has been under attack since the 1970s (cf. Binstock, 2005). ${ }^{54}$ In 1974, psychologist Neugarten observed the emergence of a group of older adults who are "relatively healthy, relatively affluent, [and] relatively free from traditional responsibilities of work and family" (Carr \& Komp, 2011; Neugarten, 1974). Neugarten called this group the 'young-old,' and situated them in contrast to the dependent and fragile 'old-old.' In 1987, the British historian Laslett popularized this distinction by coining the term third age to define a new stage in life: a period of personal fulfilment, after retirement but before dependency and frailty set in (the latter being the stage that would come to be known as the fourth age) (Laslett, 1987 \& 1991). By formulating the third age, Laslett not only advocated against the social stigma of inactivity and indolence often associated with seniors but also encouraged seniors to pursue an active life (1991). In a similar vein, Rowe and Kahn (1987) introduced the distinction between "usual" and "successful ageing" to differentiate between desirable and undesirable forms of ageing. Successful ageing encompasses "the avoidance of disease and disability, the maintenance of high physical and cognitive function, and sustained engagement in social and productive activities" (Rowe \& Kahn, 1997). The vital, active, autonomous, and healthy senior became a role model

\footnotetext{
${ }^{54}$ Since its inception at the turn of the 20th century, gerontology as a scholarly field has developed into a highly specialized discipline, encompassing many subdisciplines (such as biology, psychology, and cognitive sciences) and subfields (such as social gerontology, critical gerontology, biogerontology, and environmental gerontology).
} 
for ageing well (De Lange, 2011). What comes to count as good in this view of ageing is the delaying of disease, pushing dependence and frailty forward to the fourth age - or what gerontologists commonly describe in biomedical terms as "compression of morbidity" (Fries, 2002).

Neugarten, Laslett, and Rowe and Kahn justified their new distinctions by referring to seniors' increased longevity. In the past 150 years, the life expectancy of both men and women (in Western, affluent countries) has almost doubled. In 2016, Dutch women on average have a life expectancy of 84 years, while Dutch men on average reach 78.4 years (World Health Organization, 2016). Contemporary gerontologists often use the term "third age" in a sociological or cultural sense, to characterize a new cohort of seniors that is emerging: the generation of baby boomers in the affluent West, the greying cohort of the 'youth culture' in the 1960s (Gilleard \& Higgs, 2002; Williams, Higgs, \& Katz, 2012). This generation is said to break "the mould of the modern life course," as their lives would be "marked by change, challenge and transformation" (Gilleard \& Higgs, 2002, p. 376). This class or generation, born between 1945 and 1955 (the criteria in the Netherlands), has greater education, income, and social and material security than the generations before them had at their pensioned age. At the same time, due to their increased longevity, this cohort also faces increased risks of age-related diseases (the double greying of society). Alzheimer's and other forms of dementia are especially considered topics of concern - for individuals and also for health care organizations and governments (cf. World Health Organization, 2016).

In response to this demographic shift, all kinds of normative ageing discourses have emerged within gerontology (and beyond), such as productive aging, resourceful aging, independent aging, healthy aging, positive aging, normal aging, and civic engagement (cf. Dillaway \& Byrnes, 2009; Holstein, Parks, \& Waymack, 2011; World Health Organization, 2016). Despite their descriptive appeal, in recent years the normative content of such concepts has become the subject of debates and controversies within gerontology - a field that is seen by many as "rich in data, poor in theory" (cf. Agnus \& Reeve, 2006; Birren \& Bengtson, 1988; Carr \& Komp, 2011; Dillaway \& Byrnes, 2009; Hertogh, 2013; Katz, 2013; Martinson \& Berridge, 2015).

For a long time, Aristotle's negative outlook on ageing, as characterized by decline and loss, seemed to be the dominant view. The increased longevity, along with the demographic shifts and social and economic developments that it brings, has shifted the meaning of ageing in the 20th century. Cicero's plea to 
fight ageing by supplying the "lamp of our minds with oil" seems to have emerged again in scientific guise as "successful ageing" or one of its similar counterparts. Ageing is now something that requires an activity in order to be done well. As policymakers fear a double greying of society - rising health care costs, a lack of social services, and a decreasing part of the population participating in the production process - what to do in old age, and what it means to age well, have become topical issues in both academia and popular culture. It is in this context that the plastic, ageing brain emerges as a hopeful answer.

\section{Brain Games for Seniors: rejuvenating the ageing brain}

Cognitive training software, or what we call brain games, are computerized exercises to be played on dedicated websites or using apps on a smartphone, tablet, or other handheld device. ${ }^{55}$ While there is a wide variety of brain games available on the market, a typical brain game for seniors consists of different short exercises in cognitive training: remembering matching pictures, small calculation tasks, word puzzles, or memory exercises such as visual pattern recognition tests (reproducing particular sequences or patterns within a limited time frame). Some companies redesign well-known psychological tests into 'fun' exercises. For example, Eriksen's flanker task (used by psychologists for assessing inhibition of behaviour) is used as an exercise by Lumosity to train attention (there it is called 'Lost in Migration'), while both BrainAge and Lumosity use the Stroop colour-word interference task to "calibrate" cognitive abilities such as response inhibition, attention, memory, and processing speed. The test result is often presented in the form of a distribution curve comparing the user to other gamers in the age-group (Lumosity), or the fictitious "brain age" (BrainAge). It functions as a kind of benchmark for which the game further personalizes and proposes specific exercises. Seniors are encouraged to do the little exercises daily, motivated by these test scores as well as by emails and smartphone notifications. Some games encourage competition between users online (such as Neurocampus.com) by sharing results with others or presenting 'best-of' lists featuring the names of gamers with the highest test scores.

\footnotetext{
${ }^{55}$ In this chapter, I study popular brain games designed for a general audience. I thus do not include cognitive training programs developed for specific clinical practices, nor do I include the trend of "serious gaming" in these practices (see for an overview of such games: Costa \& Veloso, 2015).
} 
While exercises on free websites such as Neurocampus.com are quite basic and simple, games from the bigger companies are designed in a more visual appealing style. Some brain games can be played for free (such as those on Neurocampus.com), but most dedicated brain games require users to purchase or subscribe to a service to access them. For reference, the latest Nintendo BrainAge game cost $€ 29.99$ to purchase, and subscription fees range from $€ 14 \mathrm{a}$ month to access BrainHQ to $€ 240$ for a lifetime subscription to Lumosity. Brain training companies may tailor their games to specific audiences, such as people with dyslexia, ADHD, mild cognitive impairment, dementia, or Alzheimer's disease, or to specific age groups, like children or seniors. The brain games under scrutiny here all have specific programmes for seniors: Nintendo's BrainAge, Posit Science's BrainHQ, PLUS Brein Trainer (the Dutch version of HAPPYneuron), Neurocampus.com, CogniFit, and Lumos' Lumosity.

Brain games disseminate knowledge of an ageing brain through advertisements and their websites. These include slogans such as "based on neuroscience," "validated by science," "clinically proven," or having "real science, real results." Brain game websites and packaging often present users with extensive information on the ageing brain. The booklet of Nintendo's BrainAge, for example, features a short introduction on the prefrontal cortex, including colorful fMRI images that aim to demonstrate that BrainAge is "an effective way to train the prefrontal cortex," since when playing the game, "many parts of both hemispheres are working very hard" (Nintendo, 2006). In contrast to this rather simplistic portrayal of neuroscience, the websites and blogs of CogniFit and BrainHQ present summaries of recent neuroscience studies on brain training and offer extensive explanations of neuroscience concepts such as cognition, neurons, and neuroplasticity.

Through brain games, a neuroscience understanding of ageing found a general and specific audience: seniors. How do brain game companies make knowledge of an ageing brain relevant for seniors? As we saw in the previous chapters, the aim of value work is to attribute societal relevance and (moral) meaning to scientific claims for a specific audience. Brain game companies make the ageing brain valuable for seniors through different strategies of value work: appealing to seniors' concerns and anxieties, articulating values through promissory rhetoric, and anticipating the normative uptake. As we will see, this value work resulted in a scholarly and public controversy - one that was not only about a commercial exploitation of scientific validity or the effectiveness of 
cognitive training software but also about what seniors should be concerned about and even allowed to hope for with their plastic, ageing brains.

\section{Preying the Grey: cognitive decline and the risk of dementia}

Brain training companies address seniors directly in multiple ways in their advertisements, through their games, and on their websites. The most common way is to appeal to the anxiety and common worry of seniors: cognitive decline. In a little booklet accompanying the Nintendo DS game BrainAge, Kawashima asks his readers: "Did you know that just like how you lose muscle mass with age, your brain function begins to decrease when you reach maturity? This happens even if you lead a normal, healthy life" (Nintendo, 2006). Daily life activities that potentially provoke seniors' concerns related to cognitive decline, such as driving a car, hearing and vision difficulties, or memorizing groceries, are linked to specific cognitive abilities that can be trained through brain games. Lumosity and Brain $H Q$, for example, offer exercises to train memory, (brain) speed, attention, and flexibility, as well as exercises that purport to improve people skills, navigation, and intelligence.

While many companies explicitly state that their product is meant for "normal age-related cognitive decline" (BrainHQ), still they appeal to the concern for age-related conditions. CogniFit, for example, offers tailored training programmes for people with memory loss, mild cognitive impairment (MCI), dementia, Alzheimer's, and Parkinon's disease (amongst others). Regarding memory loss, we read that "there are always risks that early brain degeneration can appear, and that's why we must train our brain and not let our abilities like memory, attention, or perception be affected" (CogniFit, 2016b). Similarly, under the headline "Why train your brain?" on the website of PLUS Brein Trainer (the Dutch version of HAPPYneuron), we read about the need to "fight ageing" and that research has shown that people who do intellectually stimulating exercises have a reduced risk for Alzheimer's - implying, of course, that using PLUS Brein Trainer would be an excellent way to get such exercise (PLUS Brein Trainer, 2016).

By mobilizing seniors' concerns and anxieties surrounding cognitive decline, advocates can present brain games as an intervention strategy that can help prevent those fears from being realized - or at least keep them at bay a little longer. Fears of age-related conditions and neurodegenerative diseases become linked to general anxieties regarding age-related cognitive decline, such as memory loss, processing speed (and slower reaction time), and decreased atten- 
tion. Brain games thus articulate a familiar worry amongst seniors: that any example of age-related cognitive decline they may have experienced, no matter how common, could be a precursor to the onset of more serious age-related conditions such as Alzheimer's or other forms of dementia. Brain games are then presented as empowering seniors by giving them a means for dealing with this concern.

\section{Promising Youth and Hipness: brain games as rejuvenating the ageing brain}

The ageing brain is presented by brain game companies as plastic and amenable to change. Plasticity in the promises of brain game companies is described through the notion of "cognitive reserve" of the ageing brain. Cognitive reserve is described on websites of brain games as "the compensation of the natural decline of brain cells" accomplished "by mobilizing alternative nerve networks" (PLUS Brein Trainer, 2016), or as we can read at the website of HAPPYneuron:

These reserves represent the increase in neurons and synaptic connections (as opposed to what was believed so far, new neurons can be generated at any age). Furthermore, this reserve also represents an active process of neural plasticity allowing to optimize a person's performances either by calling upon other brain regions or by adopting new cognitive strategies. (HAPPYneuron, 2016)

By training the brain through brain games, seniors are said to be able to battle the diagnosis of cognitive decline by actively increasing their cognitive reserve.

The promises of brain training are twofold: Brain games suggest that seniors can not only reduce, delay, or even prevent age-related effects on cognition and conditions such as dementia and Alzheimer's disease but also maintain or even improve their youthful, cognitive performance. Many of these promises are dressed in suggestive, implicit, and cautious language. For example, BrainAge promises to prevent "worsening of cognitive impairment" by helping to "develop the resistance against decline in later life" (Nintendo, 2006). According to Brain $H Q$, you can "engage your brain in ways that maintain and even improve your mind into older age" (Merzenich, 2010). Referring to a longitudinal trial study (ACTIVE), BrainHQ claims that the study shows that one of its exercises "cuts the long-term risk of dementia nearly in half" (Posit Science, 2016b).

Regarding brain games' promises to maintain or enhance cognitive capabilities, Cognifit's exercises, for example, would result in improved memory, quicker thinking, faster reaction time, enhanced listening, and sharper vision. 
BrainHQ promises to "help people think faster, focus better, and remember more" as it "turns back the clock on your memory loss by about 10 years" (CogniFit, 2016c; Merzenich, 2010; Posit Science, 2016a). Benefits of a healthy brain are explained in terms of "brain health." Brain games are said to make your brains young, sharp, agile, strong, fit, bright, dynamic, and fresh. The programmes by CogniFit "boost brain power" as they inspire "the creation of new synapses and neural circuits able to reorganize and recover the function of the damaged area and compensatory transmission capabilities" (CogiFit, 2016a). The key is "to keep your neurons active," to "form new connections between brain neurons, strengthen neural networks and . . . regenerate new neural pathways" (CogniFit, 2016a). Brain health is seen as a necessary condition for well-being and autonomy at a later age, as "maintaining a high quality of life requires a sharp brain" (HAPPYneuron, 2016).

Through this promissory rhetoric, brain games enact and articulate different but related value registers: values that pertain to health and prevention, and values that pertain to youth and enhancement. In addition, advertisements and discourse on brain games employ values of enjoyment and hipness. Brain games are designed to be attractive and entertaining. Brain games are described as fun and challenging, consisting of exercises that are "original and fun but efficient" (PLUS Brein Trainer, 2016) or are "fun way[s] of making you clever" (De Jongh \& Risdale, 2010). In public discourse, we read that brain games contain "healthy puzzles for hip seniors" (De Haan, 2006). Brain game websites show pictures of laughing and delighted grey-haired seniors causally holding their iPads. Seniors in brain games are thus cast not only as proto-patients who are anxious to maintain their youthful cognitive performance but also as modern, hip, and fun-seeking.

\section{Controversy on Brain Games: contesting age-related plasticity}

The promises of brain game companies resulted in a scholarly outcry in the form of an open letter in 2014 followed by a court ruling against two brain training companies, Lumos and LearningRx, two years later. ${ }^{56}$ In 2014, the Stan-

\footnotetext{
${ }^{56}$ In January 2016, the brain training company Lumos Labs, creators of the popular brain game Lumosity, were charged by the U.S. Federal Trade Commission with a staggering $\$ 50$ million (and settled to pay $\$ 2$ million) for preying on the fears of the elderly for age-related cognitive decline, and falsely promising that their games could delay "cognitive impairment associated with age and other serious health conditions" and even stave off dementia and Alzheimer's disease (FTC, 2016a). In May of the same year, the company LearningRx faced the similar accusations by the FTC (FTC,
} 
ford Centre of Longevity together with the Max Planck Institute for Human Development issued a 'consensus letter' that was signed by some 70 scholars cognitive psychologists and neuroscientists who study human cognitive aging. The scholars in the letter questioned the real-world relevance of brain games, claiming that, "to date, there is little evidence that playing brain games improves underlying broad cognitive abilities, or that it enables one to better navigate a complex realm of everyday life" ("Consensus," 2014). The critics of brain games refer to a well-known distinction used in research on brain training: the difference between the cognitive tasks brain games challenge, and the cognitive abilities that underlie these tasks. Real "cognitive vigour," they state, would apply to this broader set of cognitive abilities, which are - in contrast to taskspecific ones - applicable in daily life. In other words: Seniors would train themselves to master BrainAge but would not train themselves to remember their grocery lists (which requires underlying cognitive abilities such as shortterm memory, reaction speed, and/or attention).

In their argument against brain games, the critics refer to "opportunity costs" in the form of alternative action programmes: instead of spending time and money on brain games, seniors could better spend their time "reading, socializing, gardening, exercising, or engaging in many other activities that may benefit cognitive and physical health of older adults" ("Consensus," 2014). The core problem for these critics is how brain games value age-related plasticity, and the malleability of cognitive health it implies. Since a scientific consensus is lacking about the extent the training of cognitive tasks can be transferred to real-world situations, the critics question the potential of the age-related plasticity of the brain. A focus on plasticity as a magic bullet for battling the effects of cognitive ageing would "detract from the message that cognitive vigour in old age, to the extent that it can be influenced by the lives we live, reflects the longterm effects of a healthy and active lifestyle" ("Consensus," 2014).

Several months after the Consensus Letter, a group of 133 international scientists and practitioners, initiated by Michael Merzenich (co-founder and chief scientific officer of Posit Science and well-known neuroplasticity pioneer), responded with a different open letter. While they concurred with the critics about the dangers of inflated promises of many brain game companies, they emphasized the potential of plasticity for the ageing brain. The proponents

2016a). While such claims were not made for companies (or their branches) in the Netherlands, the rulings were widely reported upon. 
invoked the novelty of their interventions and studies and attacked the critics for defending an "old paradigm" and "worldview" that hindered them from accepting the "full implications" of lifelong brain plasticity (Merzenich, 2014; Mahncke, 2016). Michael Merzenich calls the statement from the Stanford Centre and the Max Planck institute "irresponsible," as it "causes real harm by discouraging use of validated exercises by people who could benefit from them" (Koenig, 2014).

What's at stake in this controversy is not only scientific validity or commercial exploitation. The opponents and proponents of brain games differ in their valuation and perceived degree of plasticity of the ageing brain and the kind of malleability of cognitive health this implies. Is cognitive vigour at old age something that can be trained, or is it rather the result of lifelong experiences? In other words, what can and should seniors do with their plastic, ageing brains, and what are they allowed to hope for? To this day, the debate still rages on. New meta-analyses of brain training studies occur regularly (see for instance Kueider, Parisi, Gross, \& Rebok, 2012; Lampit, Hallock, \& Valenzuela, 2014; Melby-Lervåg \& Hulme, 2013; Simons et al., 2016) and are widely reported upon in mainstream media. Due to this controversy, the tone of Dutch public discourse on brain games for seniors shifted from "hopeful message from the neurosciences" in 2005 to a "hoax" and "waste of time" in 2015. In advice literature and popular science, the controversy on brain games appears to serve as a catalyst for neuroscientists, science journalists, and gerontologists to articulate different action programmes and ideals of ageing well.

\section{From Fragile to Agile: repertoires of an ageing brain}

Ever since the introduction of Nintendo's BrainAge in 2005, there has been a growing public interest for knowledge of the ageing brain. In the past years, a host of popular science books and advice literature on the ageing brain appeared - such as Goldberg's De Wijsheidsparadox [The Wisdom Paradox] (2006), Sitskoorn's Lang Leven de Hersenen [Long Live the Brain] (2008), Cozolino's The Healthy Aging Brain (2008), Merzenich's Soft-Wired (2010), De Lange's Breinbewust Leven [Brain Conscious Living] (2012), Van der Zee's Het Vitale Brein [The Vital Brain] (2012), and Aleman's Het Seniorenbrein [Our Ageing Brain] (2014). In this self-help and expository literature, and across accompanying discussions in newspapers and magazines, brain games seem to feature as an ethical yard- 
stick: they are often mobilized by ageing-brain advocates to position themselves in relation to, and to counter-propose different diagnoses, courses of action, and views on ageing. Through concern-making, promissory work, and coordinating the uptake, two therapeutic repertoires emerge about the ageing brain. They both mobilize the plastic, ageing brain to articulate suggestions for seniors to improve their ageing lives. Similar to the issues at stake in the scholarly controversy on brain games, these therapeutic repertoires of ageing evaluate agerelated plasticity differently, resulting in different concerns and diagnosis and different promises in terms of action programmes and ideals of ageing well.

\section{Therapeutic Repertoire I: Resisting a rusty brain}

The first repertoire bears many resemblances to the concerns and promises as mobilized by brain game companies. The main concerns in this repertoire are the effects of age-related cognitive decline for individual seniors and the risk of developing age-related conditions and neurodegenerative disorders such as mild cognitive impairment, Alzheimer's disease, or other forms of dementia. Specific worries of age-related cognitive decline concern memory loss, lack of focus and attention, and slower reaction time (processing speed), as well as the daily problems associated with these aspects of cognitive decline, such as driving a car or remembering people's names. Advocates in this repertoire downplay the promises of brain games to prevent age-related cognitive decline and propose a variety of physical exercises as alternative action programmes. What these action programmes share with a neuroscience understanding of ageing as portrayed through brain games, however, is the diagnosis of the ageing brain and the ideals seniors could and should strive for.

The diagnosis of cognitive decline is explained in this repertoire as a result of neural deterioration. As described by Dutch popular neuropsychologist André Aleman, the ageing brain faces an "irreversible decline" as "some brain cells shrink, connections between different areas of the brain disappear, memory and concentration erode, and other cognitive abilities slow down" (2014, p.8). In Soft-Wired (2013), Merzenich adds:

Just about every aspect of our brain's operations - its recording, interpreting, identifying, deciding, and reacting - slows down. Just about every aspect of a brain's accuracy - precise responding, agility, fluency, and reliability - slowly deteriorates. More often than not, that gradual decline is accompanied by a loss of confidence, a slow retreat from full engage- 
ment with the rapidly changing world, and a slow social withdrawal back to a more egocentric personal life. (Merzenich, 2013, p. 62-63)

This diagnosis of a shrinking and degrading brain points to structural changes that occur when our brains grow older. Apart from the loss of neurons and neural connections (brain atrophy), other structural changes that are often mentioned in this repertoire are white matter deterioration, decrease in myelin, amyloid deposition (the growth of plaques), and dopamine receptor depletion (Van der Zee, 2012). This bodily decline affects the functional development of the ageing brain. Faculties such as memory and executive functions decline, making the cognitive processes that co-ordinate, control, and manage our behaviour more difficult (Aleman, 2014, p. 16). Memory loss, lack of focus and attention, and slower reaction time and processing speed are characteristic of older brains and can lead to practical problems related to driving, following conversations, or social withdrawal (Aleman, 2014, p. 19; Merzenich, 2013, p. 62-63).

Luckily however, next to this "lousy status" of the deteriorating old brain (Merzenich, 2013, p. 41), the diagnosis of the senior brain in this repertoire consists of a plasticity in the form of a cognitive reserve. Actors in this repertoire often refer to studies that have found that the activity of the prefrontal cortex actually increases rather than decreases when seniors age. This would indicate that the ageing brain has a compensation mechanism to counteract the effects of neural decline. This compensation mechanism is described by the so called posterior-anterior shift in ageing, or PASA (cf. Davis et. al, 2008).

the anterior part of the brain, which we learned earlier is the most affected by ageing in terms of the structure and function of brain tissue, has to draw on all its resources in order to perform well. Calling more on this area helps older people to get the most out of their brains. In many, this process is automatic and unconscious, but it is quite possible that training our mental skills could reinforce this pattern. (Aleman, 2014, p. 38)

PASA would explain the counterintuitive finding that seniors show an increased activity in the prefrontal cortex compared to younger people, which has been presented as evidence of the ability of the ageing brain to compensate for neural decline. Cognitive reserve is mainly described in neural terms, indicating structural adaptation (Van der Zee, 2012, p. 172; HAPPYneuron, 2016). It would be determined by "the amount of oxygen made available to the brain through the bloodstream, the density of neuronal connectivity, the speed of electric sig- 
nal transmission along the axon, the concentration of critical neurotransmitters in the synapse, or the combination of all of the above" as well-known neuropsychologist Elkhonon Goldberg describes in his popular book The Wisdom Paradox (Goldberg, 2006, p. 92). Cognitive reserve in this repertoire entails the forming of new neurons and connections (neurogenesis, especially in the hippocampus), and the compensating mechanism in the prefrontal cortex (PASA). Thus, the diagnosis of the ageing brain in this repertoire is twofold: there is a natural, developing process of neural decline (resulting in cognitive decline), but the ageing brain is plastic enough to allow for compensation of this decline, notably by increasing the neural resources for cognitive reserve. Where cognitive decline is characterized by a brain that changes itself (agency attributed to the brain), cognitive decline indicates that individual subjects can influence this process. Cognitive reserve thus returns agency (who can change the brain) back to the individual subject.

This compensation mechanism is heralded as a hopeful and empowering message for seniors facing cognitive decline: seniors "don't have to fearfully watch how we mentally decline" and "don't have to helplessly think that this is the way we are" as a science journalist in a popular psychology magazine paraphrases Merzenich's message (Van der Neut, 2007). The compensation mechanism would allow seniors to actively support and reinforce it by increasing their "cognitive reserve." Action programmes in this repertoire are geared towards stimulating and challenging the ageing brain - the famous use it or lose it adage. However, what counts as a challenging activity for stimulating the brain is subject of debate. Many sceptical voices in the public debate who criticize the efficacy of brain games suggest physical exercises as the 'really proven' alternative. In her book, Breinbewust Leven [Brain Conscious Living] (2012), science journalist Brigitte de Lange suggests physical exercise as an alternative to brain games:

The good news is that you can throw those boring computer games out of the window. . . . The bad news is that you really have to get off that couch in front of the TV, or that chair behind the computer, to keep your brains healthy. Physical exercise is the most important part of a brainconscious lifestyle. (De Lange, 2012, p. 10)

While many proponents of physical exercise and other engaging activities critically debunk brain games, they do seem to piggyback on the popularity of these games by invoking similar structural brain characteristics as justification for 
physical exercise. Both brain games and physical exercises are geared towards the same goal: fostering neurogenesis, strengthening connections, and thereby improving or increasing the cognitive or brain reserve of seniors. Few promise that physical exercise actually prevent age-related conditions - at best they can delay cognitive decline and the onset of dementia. Similar to the promises of brain game companies, promises are articulated in terms of youthfulness, prevention and "brain health."

Physical exercise has "the strongest evidence," as it increases the blood flow in the brain, which is said to stimulate the release of brain-derived neutrophic factor (BDNF), activate and increase the size of the prefrontal cortex and cingulate cortex, and stimulate neurogenesis in the hippocampus (Aleman, 2014, p. 71; Van der Zee, 2012, p. 202). Engaging in regular physical exercises would result in "15\% to $20 \%$ better brain functioning" (Rozendaal, 2012). According to well-known Dutch neuropsychologist and exercise-advocate Erik Schreder, physical exercise puts the brake on age-related cognitive decline by keeping the prefrontal cortex functioning as "the seat of independence" (Brandt, 2011). Physical exercises lead to a "bigger hippocampus" and "more brain mass," which protect against dementia (De Lange, 2012, p. 123).

Various other physical exercises are proposed in this repertoire, such as dancing, playing music, travelling, bicycling (preferably not on the popular ebike), cooking, or gardening. These forms of exercise are deemed beneficial for seniors not because they prevent stiff limbs or because they are fun or even socially worthwhile, but foremost because they challenge and stimulate the cognitive performance of the ageing brain, allowing for the growth of new neurons and the forging of new connections. Through physical exercises, seniors in this repertoire are encouraged to actively stimulate their brains on a daily basis, with the goal of delaying cognitive decline and age-related conditions such as Alzheimer's and to maintain a youthful cognitive performance. Ageing well in this repertoire thus means above all staying as cognitively healthy as possible through a variety of physical exercises.

In what society or environment do brain health, cognitive health, and youthful performance of seniors become important virtues? The worry of not being able to cope with daily activities that require cognitive skills, such as driving a car or shopping, are articulated as major concerns for seniors in this repertoire. These concerns imply the ideal of self-reliance and autonomy. The plastic, ageing brain is presented in this repertoire as a form of empowerment, the ability 
to change ageing brains through physical exercise in order to keep on doing those daily activities and remain self-reliant.

Self-reliance is at times linked to a "fast-paced information society" (Aleman, 2014, p. 9) but also gains importance in the context of a declining Dutch welfare state, where seniors are encouraged to live at home as long as possible (instead of moving into a nursing home or elderly home) and to organize informal care. The societal impact of age-related cognitive disorders such as dementia has been prominent on the Dutch public agenda in recent years, becoming the most visible indicator for the danger of rising health care costs due to a double-greying society. Some speak of a dementia epidemic requiring a "dementia delta works" (Vermeulen, 2015). ${ }^{57}$ In such a context, maintaining one's cognitive skills becomes paramount, not only for the individual senior to maintain their autonomy and self-reliance and to reduce the burden of informal caregivers but also to reduce societal health care costs. Presenting the ageing brain not as a matter of fate but to some extent malleable by seniors themselves fits a welfare state where dependence on societal arrangements is discouraged.

\begin{tabular}{|c|c|c|c|}
\hline \multicolumn{4}{|c|}{ Therapeutic repertoire I: Resisting a Rusty Brain } \\
\hline & \multicolumn{2}{|l|}{ SELF-KNOWLEDGE } & \multirow{2}{*}{$\frac{\text { PRACTICAL OPTIONS }}{\text { Action programme }}$} \\
\hline & Diagnosis & Ideals & \\
\hline SELF & $\begin{array}{l}\text { Cognitive reserve can } \\
\text { counter neural } \\
\text { deterioration }\end{array}$ & $\begin{array}{l}\text { Brain health, cognitive } \\
\text { health, youthful } \\
\text { performance }\end{array}$ & $\begin{array}{l}\text { Variety of physical } \\
\text { exercises }\end{array}$ \\
\hline ENVIRONMENT & \multicolumn{2}{|c|}{ Declining welfare state, rising health costs } & $\begin{array}{l}\text { Ageing with individual } \\
\text { control }\end{array}$ \\
\hline
\end{tabular}

Figure 9. First therapeutic repertoire of an ageing brain. In this repertoire, seniors are encouraged to prevent and delay cognitive decline, and maintain their youthful cognitive performance, by training their cognitive reserve through physical exercises.

\section{Therapeutic Repertoire II: consoling a wise brain}

The second therapeutic repertoire tells a different story about the ageing brain. While this repertoire is also presented as a story of empowerment, it's an empowerment based on a different valuation of age-related plasticity, with different concerns to tackle and different ideals of ageing well. Here too, seniors are

\footnotetext{
57 The term "delta works" (or in Dutch: deltaplan) refers to the famous Dutch water works protecting parts of the Netherlands from the sea. The Dutch deltaplan was initiated after the North Sea flood in 1953 and was only officially finalized in 2010. The term thus denotes something with an extremely big impact on society which requires a long-term commitment.
} 
encouraged to escape the story of decline and loss - not because they are now deemed capable of training their brain to compensate for this decline but rather because their changing, ageing brain exhibits qualities that benefit the ageing senior as well as society at large.

The main challenge here is not cognitive decline itself but rather the stereotypical image of the senior that implies it, and the effects this has on both the self-understanding of seniors and their societal position. As a critical response to the importance attributed to concerns about cognitive decline, both by brain game companies and seniors alike, in this repertoire the very concern and diagnosis of cognitive decline come into question. Opponents of brain games call the fear of inevitable dementia the "biggest misconception regarding ageing" (Aleman, as cited in Degryse, 2013, p. 68). The advertisements of brain game companies are seen by these opponents as only adding fuel to the fire by actively stimulating the concerns and complaints of the elderly. Cognitive decline is seen in this repertoire as myth, negatively affecting seniors' well-being and serving only as an obstacle that hampers seniors from flourishing at old age (Gerritsen, 2014, p. 3; Ramscar et al., 2014).

The ageing brain in this repertoire is not so much suffering from deterioration, or not characterized by a pathology, but is rather seen as being in the process of adaptation. Comparisons with young or adolescent brains are often made in this repertoire, as ageing brains can be seen as undergoing similar processes of functional "reorganization" (Ritsema, 2012). Although not denying some structural-neural decline of the ageing brain, actors here do not evaluate this as negative, nor do they see it as the defining characteristic of an ageing brain. Instead, they draw attention to the positive effects this reorganization would amount to, especially regarding emotion regulation, memory loss, and reaction time (processing speed). Older brains would exhibit specific and desirable characteristics, especially compared to younger brains. The malleability of the ageing brain in this repertoire is limited: agency is mostly attributed to the changing brain itself, while seniors' influence on this ageing brain is considered minimal.

The ageing brain is said to be better at emotion regulation, making seniors more emotionally stable than their younger counterparts. The perceived increase in prefrontal cortex activity among seniors - also referred to in the previous repertoire - along with a decreased dependence on dopamine would explain this. According to Margriet Sitskoorn, a Dutch neuropsychologist who writes popular, expository works on neuroscience: 
With older people, there is more activation in the area at the front of the brain, the anterior cingulate cortex. This area is able to suppress the functioning of the amygdala, by which you experience emotions less intensely in certain situations. These findings support the idea that older people are indeed better capable of regulating their negative emotions, and thereby improving their feeling of well-being. (Sitskoorn, 2008, p. 10)

Older people are "better at calming the amygdala" than younger people (Aleman, 2014, p. 27). Seniors are thus said to be less affected by negative emotions; the ageing brain "keeps getting better in forgetting negative things" since their amygdala "has strong connections with the frontal lobe (where emotions are regulated) but less connections with the hippocampus" (De Jongh \& Ridsdale, 2010). Seniors would therefore remember positive experiences more easily, while at the same time they are less affected by negative experiences. As the balance shifts toward positive emotions, "you feel more happy and content" (Goldberg, 2006, p. 133). This skill of "positive emotion regulation" and "optimizing your emotional well-being" would increase when growing older (Sitskoorn, 2008, p. 9-10).

A decline in speed of information processing (the cognitive ability most brain games try to improve) is also interpreted here as a positive feature of the ageing brain. It is seen to result in better social and emotional functioning, decision-making, and problem solving. A slower brain makes seniors react less impulsively, and "because it takes longer for them to arrive at a decision, they have more information on which to base it" (Aleman, 2014, p. 81). Seniors are therefore described as sensible, less impulsive, knowledgeable, and able to make complex decisions intuitively (Van Wijngaarden, 2012).

The slower processing speed of seniors is explained by the fact that "grey cells contain a sheer amount of knowledge, accumulated over decennia" (Gerritsen, 2014, p. 3). While short-term and episodic memory use becomes more difficult, the use of existing memories, knowledge, and experience would be improved. Due to a shift in sub-regions in the hippocampus, the ageing brain would be better at retrieving earlier associations and memories (Van der Zee, 2013, p. 163-164). Goldberg (2006) speaks of the increased ability of the ageing brain to "recognize mental patterns" based on experiences and the accumulation of "generic memories" through our lifetime (Goldberg, 2006, p. 124). As a result, seniors are said to be better at seeing the big picture and drawing abstract associations. 
This amounts to a diagnosis of the ageing brain not as a "damaged brain with broken parts" but as a brain that is "functionally organized" differently with particular strengths and "vitality" as the title of Dutch molecular neurobiologist Van der Zee's popular expository book refers to (Van der Zee, 2012, p. 213). In contrast to a brain characterized by slow decline and lack of cognitive performance, the ageing brain is presented as being more efficient and flexible. Underlying this diagnosis is a view of age-related plasticity as a form of compensation. A different theory of compensation is used than in the first repertoire. Instead of the notion of cognitive reserve or the theory of PASA, actors in this repertoire often refer to the model of hemispheric asymmetry reduction in older adults, also known as the HAROLD model (Cabeza, 2002). The HAROLD model posits that the two hemispheres of the brain function differently in later age. In the ageing brain, the two hemispheres are said to "co-operate" better together, resulting in less cognition and emotion lateralization and a better integration of emotion and cognition (Aleman, 2014, p. 81; Cozolino, 2008, p. 108-110; Goldberg, 2006, p. 124; Sitskoorn, 2008, p. 19). While younger people mostly rely on one hemisphere at a time in decision-making processes, seniors would draw support from both hemispheres to compensate for age-related decline. While the right hemisphere is said to shrink, the left one shows "greater resilience," allowing seniors to "deal efficiently and effectively with familiar situations of mental routines" (Goldberg, 2006, p. 113).

As a result, the ageing brain in this repertoire enables seniors to be better in decision-making than their younger counterparts, especially in complex social and emotional situations. The better integration of cognitive and emotional functioning is seen to be the result of "older people learning to assess the value of both reason and emotion, and to give each its own place" (Aleman, 2014, p. 81). The change in hemisphere asymmetry turns the ageing brain in this repertoire into a wise brain, described in virtues such as emotional stability and equanimity, peace of mind, a balanced personality, empathy, flexibility, ability to self-reflect, subservience and generosity, helpfulness, being knowledgeable, and having expertise, life experience, and (historical) perspective. Compared to the "impetuous and egocentric ambition of youths" (Van der Sman, 2006), the ageing brain is considered by many as a better brain than its younger version.

What should seniors do with their wise brains? The ability of the ageing brain to compensate for itself due to lifelong experiences is often described as a self-regulatory ability or "mental autopilot." At the same time, the wise brain does come with some prescriptions and action programmes. According to 
Goldberg (2006), only people whose mental lives have been "both vigorous and rigorous" can attain a "mighty coat of mental armour" in their later years:

This armour, a mental autopilot of sorts, will serve them in good stead during the final decades of their lives. This mental armour, the rich collection of pattern-recognizing attractors in the brain, is not an entitlement and its attainment in old age is not a foregone conclusion. It is a reward for the vigorous life of the mind in younger years. (Goldberg, 2006, p. 160)

Long before we reach the older years, we have to start building our compensatory mental armour or wisdom. Instead of promising the short-term results of cognitive training or suggesting seniors exercise daily to maintain a healthy brain, actors in this repertoire emphasize the long-term effects of staying active and engaged throughout our adults lives. Even after lifelong learning, a wise brain should not be seen as an "entitlement" or a "foregone conclusion":

while taking full advantage of mental autopilot, one must not allow oneself to be lulled by it. Regardless of one's age, one must continue to test one's mind and strive for new mental challenges. (Goldberg, 2006, p. 161)

The ageing brain still needs some stimulation, then, in order for seniors to receive the gift of wisdom. For seniors already in their older years, suggestions in this repertoire pertain to an active lifestyle. Most proponents don't see the need for specific action or training programmes, as our "information society provides enough stimuli to put our brains to work" (Jolles, as cited in De Lege, 2007, p. 5). Or, as Dutch psychologist and brain game critic Douwe Draaism puts it: "The only thing you can do is keep using it [the brain]. That doesn't take extreme efforts. An active social life will do" (Draaisma, as cited in Kuiper, 2008, para. 4-5).

Seniors are told in this repertoire not to worry too much about cognitive decline. Actors argue that many complaints are not proportionate to an actual decline of memory in practice (Draaisma, as cited in Oden \& Hald, 2008, p. 88; Olde Rikkert, as cited in Evenblij, 2003). Complaints about memory loss by 60year-olds are seen as the result of insecurity and fear, encouraged by ghost stories propagated by the advertisements of the brain game industry. Seniors should not "problematize the normal, daily forgetfulness" - instead, they should get used to mild forgetfulness and "accept the inevitability of ageing" (Degryse, 2013, p. 68; De Lange, 2012, p. 132; Pek \& Van Osnabrugge, 2013; Van 
Hintum, 2009). While these arguments are often meant as consoling, some actors use them to critically characterize seniors who play brain games as the worried well, a characterization (in this case) especially of the cohort of baby boomers who are "bright, aging, concerned, and motivated to act on their concerns" (Goldberg, 2006, p. 152).

Fear of cognitive decline could actually result in real memory loss - a selffulfilling prophecy, as "the more you think you suffer from memory loss, the less effort you will take to remember something" (Draaisma, as cited in Oden \& Hald, 2008, p. 88). Worrying about cognitive decline would have "a bad influence on the process of ageing" (De Lange, 2012, p. 135), while the effect of a positive outlook on ageing is presented as bigger than the effects of lifestyle factors such as physical exercise, smoking, or obesity (Aleman, 2014, p. 10; Degryse, 2013, p. 68). Seniors are thus encouraged in this repertoire to stay optimistic and develop a positive attitude towards their own ageing. They are encouraged to "feel proud" of their ageing brains (Oden \& Hald, 2008, p. 88).

Next to improving the self-image of the ageing senior, action programmes in this repertoire are also geared to the social well-being and societal status of seniors. Seniors should "nurture their relationships," as positive social support is beneficial for mental health, the reduction of stress, and increasing well-being "by feeling cared for and esteemed by others" (Cozolino, 2008, p. 234). Seniors should make sure they keep fulfilling a societal role, for example by volunteering or delaying retirement, as working after the age of 65 is "a hidden blessing" for the ageing brain. Given their unique, wise brains, actors in this repertoire plea for a rehabilitation of seniors in society:

The special status and privileges that seniors in some countries enjoy, are hard to find here. In some countries, seniors are honoured for their wisdom. We only have a $60+$ card of the Dutch railways, and we are told not to complain too much. (De Lange, 2012, p. 131)

Based on their knowledge, experience, and wise brains, some advocates propose that seniors should not just try to continue working but should be put in charge of important decision-making processes, such as in politics or in corporations (De Vries, 2012). Increasing seniors' status in society works both ways: It would be good for society, and it would also be beneficial for the ageing brain, as "senior brains benefit from a society that values older age" (Haijtema, 2012, p. 2). 
The image of ageing well that emerges in this second repertoire is one of a wise senior who has led a vigorous life of the mind, stays socially active and intellectually engaged, and has a positive attitude towards ageing. As we have seen, the emphasis shifts towards positive aspects of ageing, as actors debunk "the myth of cognitive decline" and replace it with a diagnosis of gains. Whereas values of youth and performance were part of the ideal of ageing well in the first repertoire, here youth is used as an anti-position: Ageing brains are presented as essentially different and better than their younger counterparts. Instead of resisting ageing, seniors should accept and cherish it, as it comes with the virtue of wisdom. In contrast to the individual form of empowerment of the first repertoire, the wise, ageing brain is mobilized here to rehabilitate seniors' self-image and the position of seniors in society.

A wise senior needs a particular society to flourish in. Advocates of the ageing brain in this repertoire, shown in Figure 10, often invoke the knowledge economy to explicate the added societal value of seniors. In recent years, the retirement age of Dutch seniors has steadily been increased to 68. The employability of seniors is a recurring topic in Dutch public discourse, where not only the benefits of seniors for corporations are discussed but also the problems seniors have finding or maintaining jobs in the face of unemployment and the bias towards younger employees in the job market. In this context, advocates often argue that a knowledge economy should value seniors not only for their knowledge and experiences but also for their qualities such as wisdom and decision-making skills. Ageing brains hold a "treasure of experiences" (Bakker, 2008, p. 14), and the myth of cognitive decline means a waste of "human potential and social capital" (Gerritsen, 2014, p. 3). Working longer, volunteering, or otherwise contributing to a knowledge economy would not only be beneficial for keeping seniors' ageing brains fit, but it would also serve society at large. While ageing itself is presented as less malleable here than in the previous repertoire, a greater role is attributed to the environment for contributing to seniors ageing well. 


\begin{tabular}{|c|c|c|c|}
\hline \multicolumn{4}{|c|}{ Therapeutic repertoire II: Consoling a Wise Brain } \\
\hline & \multicolumn{2}{|l|}{ SELF-KNOWLEDGE } & \multirow{2}{*}{$\begin{array}{l}\text { PRACTICAL OPTIONS } \\
\text { Action programme }\end{array}$} \\
\hline & Diagnosis & Ideals & \\
\hline SELF & $\begin{array}{l}\text { Functional reorganization } \\
\text { comes with gains }\end{array}$ & $\begin{array}{l}\text { Wisdom, emotional } \\
\text { stability, well-being }\end{array}$ & $\begin{array}{l}\text { Lifelong (cognitive) } \\
\text { engagement, positive } \\
\text { attitude }\end{array}$ \\
\hline ENVIRONMENT & \multicolumn{2}{|c|}{$\begin{array}{l}\text { A knowledge economy which needs seniors' } \\
\text { wisdom and life experience }\end{array}$} & $\begin{array}{l}\text { Environment } \\
\text { constitutive for ageing } \\
\text { well }\end{array}$ \\
\hline
\end{tabular}

Figure 10. Second therapeutic repertoire of an ageing brain. In this repertoire, seniors are encouraged to stay socially engaged, not worry too much, and embrace their unique, wise brains.

\section{Successful Brain Ageing in a Hypercognitive Society}

The two therapeutic repertoires show how the ageing brain is made valuable for seniors, and how different valuations emerged as a response to the controversy surrounding brain games. In the first repertoire, critiques against brain games led to the propagation of physical exercise as a proven alternative action programme. In the second repertoire, the critique of brain games was more substantial, as the very concern and diagnosis of an ageing brain characterized by cognitive decline is replaced by a reorganizing brain with its own desirable characteristics. While ideals in the first repertoire are described mostly in neurological idiom ("brain health" as indicator for cognitive health), ideals in the second repertoire are articulated in terms of wisdom and social and emotional well-being. In practice, the two repertoires of the ageing brain happily co-exist, build upon, and reinforce each other. Despite their differences in diagnosis, action programme, and ideals, they find each other in their use of the plastic, ageing brain to provide alternative outlooks for the 'paradigm of decline and loss,' where seniors are framed as deteriorating burdens on society. The two repertoires provide alternative stories of hope and consolation, where cognitive losses are either overcome or turned into gains.

After tracing valuations of the ageing brain in the value work of brain game companies, and learning how these valuations became contested and resulted in two emerging repertoires of ageing well with a plastic brain, we now turn to the question of neuro-moral change. Does knowledge of the ageing brain challenge our understanding of what it means to age? To what extent does the appropriation of the ageing brain result in new norms or ideals of ageing well? I explore in this section to what extent the ageing brain challenges how we 
should understand seniors, the practical options they have, and what the assumptions and implications are for understanding ageing in our contemporary society through knowledge of the plastic brain.

\section{The Cognitive Senior: from anti-ageing to positive ageism}

How does a neuroscience understanding of ageing challenge our conceptions of ageing? What aspects of growing older become fore- or backgrounded through knowledge of the ageing brain? The primary focus of the ageing brain (in both repertoires) is on aspects of cognition as defining characteristic of seniors. In both repertoires the ageing brain is heralded as a way of overcoming the paradigm of cognitive decline. Each repertoire responds in its own way, building upon and reiterating different yet traditional views of ageing. We can find traces of both repertoires already in the work of Cicero, who argued that we should resist ageing as a disease, but also that we should cherish the wisdom that comes with it. The two repertoires thus reflect the ambiguity of ageing as represented through history: as a time for facing inevitable cognitive decline while reaping the fruits of an experienced life.

There are different plasticities at play in the two repertoires. In the controversy over brain games, the indeterminate nature of brain plasticity became subject of debate: What can be changed of the plastic, ageing brain, and what should we accept as unchangeable? By invoking compensation theories (such as cognitive reserve or PASA), advocates in the first repertoire argue that structural compensation for cognitive decline could be strengthened by exercising the brain. Similar to the plasticity mobilized in the case of the mindful brain, agency is given here to the individual senior to change her brain functioning. In the second repertoire, different compensation theories (such as HAROLD) are used to establish that the plastic, ageing brain grows into an essentially different brain than its younger version. Here, agency remains located in the ageing brain itself: Seniors can only minimally affect its trajectory, and not directly through exercising the brain. The ageing brain in this repertoire is seen as the result of seniors' rich, lifelong experiences, which allow the ageing brain to compensate for cognitive decline. Similar to the case of the teenage brain, this plastic, ageing brain is presented as having unique characteristics: in this case, wisdom.

These different views on the plastic, ageing brain have consequences for what seniors are allowed to hope for and what view on ageing they implicitly propose. While both repertoires put cognitive health on center stage, the form this ideal takes differs. In the first repertoire, cognitive health is framed in terms 
of "brain health" and values of youth and prevention of illness. Ageing is presented as a process that can be delayed or (as in the case of brain game companies) as a disease that can be prevented and should be fought. The underlying view on ageing, then, is its denial: It is a view of anti-ageing. It's a traditional view on old age that has a long history (and upcoming future), as we can trace its roots from Seneca, Gilgamesh, and the Fountain of Youth, to recent posthumanist biomedical attempts to rescue humanity from its biological chains (cf. Mykytyn, 2010).

In this view, growing old should be overcome by maintaining youthful performance as long as possible. Similar to ideals of the young body, the young brain is projected unto the old brain and becomes the norm (Holstein et al., 2011). Over the years, many scholars - mostly from fields such as critical and social gerontology, feminist studies, and disability studies - have criticized such anti-ageing views. ${ }^{58}$ This view not only lacks any positive normative content of what ageing could be (apart from its opposite of being young) but it also reintroduces a form of negative ageism, supporting prejudices and discrimination against those that do not fulfil the norm of maintaining a "sharp" brain. Moreover, labelling ageing as a disease effectively blurs the distinction between 'normal' age-related cognitive decline and neurodegenerative disorders such as Alzheimer's. In other words: Every senior who sometimes experiences memory problems turns into a pre-patient, for which brain games - or physical exercises - become a necessity (cf. Egher \& Wyatt, 2016; Katz \& Peters, 2008; Millington, 2012). Arguably, this "therapeutic limbo" (Webster, 2002, p. 445) does not diminish the concern for age-related conditions such as Alzheimer's but rather reinforces it (Egher \& Wyatt, 2016).

In the second repertoire, ageing is seen not as something undesirable that needs to be combatted but rather as an inevitable process that comes with its own gains - provided one has lived a life with cognitive vigour. Here, the ageing brain is used to reiterate the virtue of wisdom. Instead of an anti-ageing view, we see here an example of positive ageism at play - prejudices in favour of older people, in this case stereotypical brain traits that are deemed beneficial for both the individual senior and society (cf. Butler, 1969; Palmore, 1999). This view of positive ageism is used to debunk two dominant positions: the antiageist values of youthful performance underlying brain games and exercises in

\footnotetext{
${ }^{58}$ For an overview of critiques on anti-ageing views, see Dilway \& Byrnes, 2009; Martinson \& Berridge, 2015.
} 
the first repertoire, and dominant negative ageist views in society (e.g., seniors as costly burdens of society). Compared to the senescent virtues of wisdom of Cicero, which included some letting go or acceptance of mortality, the wisdom of the ageing brain becomes described in neurological and cognitive terms (e.g., slower reaction time, hemispheric asymmetry reduction), turning such virtues into mere aspects of cognitive health. Still, the second repertoire shows a minor moral chang, similar to the shift in self-understanding of the teenage brain: a more positive self-understanding of seniors as being wise now has a richer biological discourse by which it gains relative weight compared to already dominant views of seniors as pre-patients who need to stay young.

What both repertoires have in common is the neuro-essentialist assumption of the ageing brain. Just like in the case of the teenage brain, the ageing brain implies that there is a single brain type, with certain biological characteristics, that would be common for all members of the category 'old,' regardless of individual differences or other differences such as sex, gender, or social-economic class (O'Connor et al., 2012). This characterization proves to be especially problematic for seniors, who have differentiated themselves (and their brains) throughout their lives - differences between seniors are thus probably even greater than within other groups (such as teenagers). In both cases, the specific brain-type of the ageing brain is presented as a form of empowerment. In the first repertoire, this empowerment is directed towards the individual senior, while empowerment in the second repertoire takes a more social form, as the ageing brain is used for a rehabilitation of the societal status of seniors.

\section{Exercising the Ageing Brain: from successful to healthy ageing}

What kind of practical options does the ageing brain afford seniors? And how do these options challenge what seniors should do to age well? The ageing brain presents ageing and ageing well as requiring some activity. As a response to brain games, action programmes in the two repertoires contain existing activities, refurbished as being beneficial for brain health (e.g., exercise, gardening) or for staying socially and intellectually engaged (e.g., prolonging retirement). These action programmes of a "brain conscious lifestyle" do not radically challenge the ways seniors can age well. Rather, both repertoires of the ageing brain reflect gerontological theories of successful, active, productive, and healthy ageing, as they have developed over the past twenty years.

Successful ageing was described by Rowe and Kahn as encompassing "the avoidance of disease and disability, the maintenance of high physical and cog- 
nitive function, and sustained engagement in social and productive activities" (1997, p. 433). Ever since the introduction of the notion of successful ageing, seniors are encouraged to socially, intellectually, and physically get active, resulting in different variants of successful ageing, such as active ageing, productive ageing, or healthy ageing. In all these variants, seniors are endowed with the agency, choice, and responsibility to take care of their own (physical and cognitive) health. Both repertoires mobilize aspects of these variants of successful ageing and encourage seniors to stay active. The difference lies in their scope and in the promise of what seniors might hope for. In the first repertoire, action programmes are short-term and geared towards improvement of cognitive functioning and maintaining "brain health" (as indicator for cognitive health). Successful ageing here means, first and foremost, exercising your brain through daily physical exercises.

In the second repertoire, action programmes combine aspects from productive ageing and healthy ageing (cf. World Health Organization, 2016). Seniors are encouraged to stay socially and intellectually active at old age - for example, by postponing retirement. In addition to staying active (in a broad sense), action programmes are geared towards prevention and lifelong learning: Only by having led a life with cognitive vigour can one reap the fruits of a wise brain. This implies that long before seniors become seniors, they should already have been working on their 'mental armour' - the foundation that allows for wisdom, well-being, and other virtues of old age to emerge. The need to be cognitively challenged thus starts during adulthood, expanding healthy ageing throughout the life course. Whereas seniors in the first repertoire became prepatients, adults in the second repertoire become pre-seniors.

From the perspective of active or successful ageing, the ageing brain does not seem to challenge existing action programmes. Brain games do seem to provide a new practical option for seniors to train their cognitive abilities. Indeed, brain games probably have helped by introducing computerized training, game consoles, and iPads to many seniors. At the same time, the content of many of these brain games - with the exception of some of the more substantial games that are based on psychological tests and theories - hardly differ from the sudoku, crossword puzzles, or board games that seniors are familiar with. More than their content, it is their digitized form (computerized games) that turn them into a new practical option for seniors to use to care for their brains.

Compared to other action programmes, these forms of neuroascesis for seniors do add something distinctive to the palette of practical options for ageing 
well. Both brain games and physical exercises are often presented to seniors as valuable alternatives for pharmaceutical interventions, as "the effect of physical exercise on cognition is just as strong as that of medicine" (Mudde, 2013). Brain games or physical exercise then become an easy, less-medicalized replacement for chemical inducements for maintaining cognitive vigour (Millington, 2012). A healthy or better functioning brain as justification for intervention has thus travelled from pharmaceutical interventions, through the discourse of brain games, to 'ordinary' daily activities of seniors, such as gardening or doing sports.

\section{The Ghost of Alzheimer in a Hypercognitive Society}

In what kind of society and environment does the ageing brain flourish? And what does the emergence of the ageing brain imply about the role of seniors in our contemporary society? Emerging from the two therapeutic repertoires is a society that puts great emphasis on cognitive health and performance. In this knowledge society, or hypercognitive society (cf. Post, 2000), maintaining cognitive health in older age becomes a mark of ageing well. In the first repertoire, the individual 'will to health' is encouraged through brain games and physical exercise, implying that keeping cognitively fit, and even attaining youthful cognitive performance, is the best way for seniors to remain valuable citizens. As many scholars have addressed, maintaining one's cognitive skills is not only an opportunity for a better life. In the context of rising health care costs and a further decline of social arrangements for seniors, building or maintaining cognitive autonomy and self-reliance can easily become a responsibility or duty.

In contrast to this perverse regime of health (cf. Lawless \& Augoustinos, 2017; George \& Whitehouse, 2011; Millington, 2012; Williams et al., 2012), the ageing brain in Dutch popular discourse also allowed for a more positive societal role for seniors. As we saw in the second repertoire, the ageing brain was used to address the societal position of seniors by showing how their distinctive brain qualities can contribute to our knowledge economy. In a society where the "impetuous, egocentric and unencumbered ambition of the youth" (Van der Sman, 2006, p. 66) is celebrated, the lifelong experiences, flexibility, equanimity, and, in short, wisdom, are presented as valuable qualities. While this social empowerment can be considered somewhat more positive than the individual encouragement to remain cognitively fit, it comes at a cost.

The ageing brain is a story that brings empowerment and control for seniors in the Third Age, where there is hope, consolation, relatively good health, and 
affluence. All features that do not fit this story are silently transposed to the Fourth Age - a period which is hardly articulated in discourses of ageing brains. Tighter control over the Third Age thus comes at the expense of control over the Fourth Age. The ageing brain thereby seems to underscore critiques of successful ageing, where the Fourth Age becomes black boxed (Holstein et al., 2011). Every senior who is not able to live up to the norms of successful (brain) ageing - by either keeping up their cognitive health or societally contributing with their wise brains - is pushed to the Fourth Age, where physical suffering and existential dread do seem to appear. The plastic, ageing brain as persuasive antidote to the paradigm of decline and loss effectively mystifies this aspect and view on old age. As frailty, fragility, senescence, dependence, and death do not fit the story of the plastic, ageing brain, this view of old age (and how to deal with it) is pushed from view, and becomes a matter of fate; silently present, looming in the corner.

At the same time, distinctions between health and illness, normal and abnormal ageing - in short, between the Third and Fourth Age - become blurred in repertoires of the ageing brain. In the first repertoire, 'normal' memory loss or other age-related cognitive decline experiences are presented as potentially leading to medical conditions such as dementia or Alzheimer's disease. In the second repertoire, wisdom and emotional stability are seen as valuable forms of cognitive health which can be attained even when experiencing memory loss or lack of attention. One can see this as either stretching the category of health or diminishing the category of illness. Either way, how to cope with cognitive decline, Parkinson's, Alzheimer's, or any other age-related condition is not a topic that is easily discussed using the plastic, ageing brain. While the inevitable horizon of old age remains unarticulated, the path to it has become slippery and wet, as adults become pre-seniors, and seniors become pre-patients. Arguably, not giving room to frailty and mortality perpetuates anxiety for the inevitable amongst seniors rather than diminishes it.

\section{Conclusion}

In this chapter, I explored how the ageing brain is made valuable in Dutch public discourse to discuss what it means to age, and how to do it well. Taking the controversy surrounding brain games as entry point, I showed how brain game companies, through strategies of value work, addressed the concerns and anxie- 
ties of seniors. As their promises of preventing age-related cognitive decline and conditions such as Alzheimer's raised a scholarly controversy, the ageing brain and the potential of its plasticity became topics of public debate. The controversial status of brain games appeared to provide science translators and science popularisers with an avenue for ethical deliberation on what it means to age well with a plastic brain. Just like brain games, the two therapeutic repertoires presented here provide alternatives for the dominant paradigm of decline and loss. However, each repertoire critically responds in its own way to the concerns and promises mobilized by brain game companies. Different valuations of the malleability of the ageing brain resulted in two different therapeutic stories on what seniors could and should do with their ageing brains, and what they are allowed to hope for.

The normative power of the plastic ageing brain allows actors to mix conflicting views on successful ageing, tapping into and re-articulating both contemporary and traditional views of anti-ageing and positive ageism, combining hope with consolation. The ageing brain therefore did not result in substantial moral changes in the practical options seniors have and what it means to age well. However, just as in the case of the teenage brain, the ageing brain did give some extra weight to a more positive understanding of seniors, which strengthened this position compared to dominant anti-ageist or negative ageist views of seniors as societal burdens or pre-patients who need to stay young. In contrast to other studies on the ageing brain and brain games (cf. Lawless \& Augoustinos, 2017; George \& Whitehouse, 2011; Millington, 2011; Pickersgill et al., 2017; Pitts-Taylor, 2010; Thornton, 2011; Williams et al., 2012), the emergence of the second repertoire shows that a neuroscience understanding of ageing also allowed advocates to address the societal status of seniors, reiterating cultural views on wisdom in terms of seniors' unique plastic, ageing brains. Both repertoires still presuppose a society in which cognition is a marker of ageing well when cognition, in the form of either cognitive health or wisdom, fails, seniors are left with an unarticulated Fourth Age.

In contrast to the previous two cases, the indeterminate nature of plasticity itself becomes an explicit object of critical scrutiny in the case of the ageing brain: What can be changed, and what should be accepted, were precisely the topics of the scholarly controversy. This resulted in two different therapeutic takes on what it means to age well with a plastic brain. Similar to the chapter on teenage brains, here too the plastic ageing brain is presented as a distinctive brain, with unique opportunities and risks (second repertoire). And just like the 
mindful brain, the ageing brain is conceptualized in the first repertoire as a brain that can still be changed through specific forms of brain training. These different plasticities and distributions of agency seem to culminate in the ageing brain. The case of the ageing brain also presents us with some limits of the normative appropriation of brain plasticity. Where too much plasticity of the teenage brain was either considered a virtue or vice, and where plasticity became a task for the working adult, a lack of plasticity for seniors seems to become a sign of the inevitable end. When agency slowly escapes us, and there is no hope left, it seems we can no longer talk about plastic brains. 



\section{CHAPTER 5}

\section{Practicing the Plastic Brain}

\section{Introduction}

$\mathrm{I}^{\mathrm{n}}$ the previous chapters, I explored valuations of a plastic brain in three different practices of flourishing. The starting point of this dissertation was the omnipresent promise that knowledge of brain plasticity brings us a new understanding of ourselves, new ways to improve our lives, and even new ideals to strive for, resulting in a "brain-based philosophy of life" (Gazzaniga, 2005, p. $\mathrm{XV}$ ). As our brains are now seen as changeable, translators and popularisers of neuroscience knowledge encourage us to change them for the better - enhancing the good and preventing the bad. Throughout this dissertation, I have scrutinized how the plastic brain was normatively used to articulate the good and bad in different contexts of the good life.

Plasticity is an equivocal and performative ethical concept: Its interactionist and indeterminist nature conceals what we can and cannot change, and who should change what. Enthusiasts celebrate its malleability and empowering potential, while pessimists fear new forms of disciplining and social control. By taking an empirical and pragmatist ethical approach, I sidestepped the debate between enthusiasts and pessimists of brain plasticity, who both share the assumption that the import of neuroscience in societal domains automatically leads to specific moral changes (empowerment versus control). Instead of assuming a transformative potential of the neurosciences, I explored how the technical knowledge of the neurosciences was made valuable by its advocates and 
translators, by addressing specific ethical challenges and ideals in practice, before I probed for possible neuro-moral changes in such practices.

I analysed the journey of valuations and normativities of three manifestations of a plastic brain, from the lab to three different domains of the good life: parenting, working, and ageing. My aim was to explicate the strategies used by actors in making brain claims valuable for specific audiences and for specific ends - strategies I named value work. In the previous chapters, I showed how different strategies of value work resulted in the emergence of multiple therapeutic repertoires of parenting, working, and ageing well with a plastic brain. Through these recurring valuations of a plastic brain, I mapped for what sorts of problems, prescriptions, and ideals the plastic brain was mobilized in these cases. By putting the therapeutic repertoires in a relevant historical context, I questioned to what extent the plastic brain challenged prevailing ideas of what it means to be a good parent, employee or senior.

The main finding of this thesis is that the plastic brain was made valuable in each case by becoming aligned with a variety of context-specific concerns, diagnoses, action programmes, and ideals - of parenting, working, and ageing well, respectively. Contrary to promises of neuro-advocates, the import of knowledge of the plastic brain in contexts of parenting, working, and ageing did not result in pronounced or substantial destabilizations of what constitutes the good in these contexts, or how to achieve it. This, however, did not mean that the practices studied were not affected by the import of the plastic brain. In some cases, we did find some destabilizations in the hegemony of moral positions, where the plastic brain allowed advocates to contest dominant positions and propose alternative repertoires.

Whereas the empirical chapters exemplify the contextual specificity of valuing plasticity and its moral changes for a particular context, in this concluding chapter I aim to understand the similarities in valuations of plasticity in practice. After summarizing the key findings in each case, I argue that the ethical ambiguity of the plastic brain, and especially how it affords value work in practice, explains to a great extent the lack of moral change found in the three cases. It enables advocates to make the brain valuable in multiple ways through a dance of agency - a dance that follows patterns that we know too well from earlier debates on the malleability of man and society. ${ }^{59} \mathrm{I}$ then reflect on the

59 The term "dance of agency" is coined by Pickering (1995), by which he described scientific practices as a back-and-forth, and swapping of roles, between humans and nonhumans: The material scientists work with tends to both resist and accommodate human actors (and vice versa). While my 
good life with a plastic brain and return to the debate between its enthusiasts and detractors. I end with a short reflection on the benefits and limitations of my approach for understanding the societal impact of the neurosciences.

\section{Changing Brains, Changing Moralities?}

The main conclusion of this dissertation is that advocates were able to make the plastic brain valuable for multiple, context-specific ideals of parenting, working, and ageing well. While neuroscientists and other neuro-translators promised a final solution to context-specific challenges, their answers proved to be inconclusive, as different brain claims of plasticity were used to articulate and cater to different (and sometimes even conflicting) therapeutic repertoires.

In chapter two, we saw that parenting teenage brains resulted in different normative suggestions regarding expectations parents should have of their adolescents, the degree of control that they should exert, and the way of exerting control. Together, the two therapeutic repertoires presented here urge parents to both control and let go. The teenage brain is presented as credible support for this difficult balancing act. However, the emergence of the two repertoires shows that the teenage brain did not provide a conclusive answer: The message of advocates mobilizing the teenage brain as parenting advice is that parents should do both. The teenage brain as parenting advice thereby did not challenge or destabilize what it means to parent well, as it was rather evaluated through current Dutch ideals of parenting: protection by setting clear limits (strong control) while acknowledging and nurturing the needs of the adolescent for self-actualization. We did find some shifts in the relative weight of particular views on adolescence and ideals of parenting: The 'vitality' of adolescence gained a new, biological underpinning ("unique brains") by which it could contest dominant views of 'troublesome teens,' and so did contemporary views on authoritative parenting.

In chapter three we saw how in the context of work, a de-ethicized mindful brain was re-ethicized in multiple ways, aligning to different concerns and ideals at the office: stress due to productivity loss or a lack of job satisfaction. Talking about productivity alone would probably not have resulted in the widespread adoption of mindfulness by employees and managers alike. By combin-

perspective is not explicitly materialist-agential, the concept fits the use of plasticity arguments in practice. 
ing a language of control and productivity with the language of empowerment, well-being, and communal life at the office, the mindful brain was celebrated by advocates as a solution to the concerns of both employees and managers. The mindful brain did not challenge conceptions of modern employees, or contemporary ideals of working well, but rather promised to fulfil the conflicting demands of our highly technologized knowledge economy: Employees could be both be relaxed and resilient, happy and high performing. Moral change in this case took the form of a shift in the relationship between ideals: Mindfulness as individual empowerment did not rule out addressing and improving the social environment at work. The promise that we don't have to make trade-offs, that we can have it all, explained the appeal of the mindful brain in the context of work.

In chapter four, we saw how brain games functioned as an ethical yardstick for the emergence of two different repertoires of ageing well. Advocates were able to use the aging brain to either contest brain games and propose alternative action programmes - e.g., physical exercise, sports, and gardening - or sidestep concerns about cognitive decline altogether by focusing instead on the aging brain's unique, beneficial traits (e.g., wisdom). While the repertoires in this case found each other in their resistance against the dominant paradigm of decline and loss represented in the emergence of brain games, each repertoire responded in its own way. Advocates were able to mobilize the plastic, ageing brain to mix conflicting views on successful ageing, tapping into and re-articulating both contemporary and traditional views of anti-ageing and positive ageism to combine hope with consolation. Substantial moral changes did not occur, as the two repertoires reflected the ambiguity of ageing as represented through history: as a period for facing inevitable cognitive decline while reaping the fruits of an experienced life. Similar to the shift in self-understanding of the teenage brain, a more positive self-understanding of seniors as being wise did gain relative weight compared to dominant views of seniors as pre-patients who need to stay young.

The emergence of multiple repertoires of doing and being good in each case is the main explanation why the valuations of the plastic brain did not result in substantial moral changes in self-understandings, ways to achieve the good life, or the ideals that we can strive for. It is precisely the combination of different self-understandings, practical options, and ideals that explain the traction the plastic brain gained in these different contexts. As a result, moral changes did not take the form of radically different self-understandings or of new means to 
achieve the good life - as promised by advocates of the plastic brain. Rather, moral change appeared as minor shifts in the hegemony of positions: in the relationship between elements of the good life, and in the relative weight of alternative moral positions. We have to keep in mind that the concept of the therapeutic repertoire is analytical: it is the result of the analysist's work of dissecting and discerning valuations and normativities in the argumentations of brain advocates. In practice, neuro-advocates combine, switch, and slip among different repertoires. This power to combine is why substantial moral changes did not occur. The plastic brain can be used for proposing different, sometimes even conflicting, diagnoses, action programmes, and ideals.

\section{Plasticity in Practice: a dance of agency}

As we recall from the introductory chapter, the problem of plasticity as ethical concept is its interactionist and indeterminate nature: It positions us, the brain bearers, in between malleability and determinism, and in between our own brain functioning and our environment. The concept of the plastic brain thereby conceals questions that were central to $20^{\text {th }}$-century nature/nurture and agency/structure debates; which aspects of ourselves or our surroundings can we consciously change, and which should we accept as unchangeable? And who should change what? Some scholars in the humanities and social sciences have celebrated plasticity as a 'peacemaker' capable of bridging the gap between nature and nurture (cf. Beaulieu, 2000; Ledoux, 2002; Mohr, 2003). However, I argue that valuation of the plastic brain in practice still seems to occur through polarities we know well from agency/structure and nature/nurture debates.

The ethical ambiguity of the plastic brain in all three cases presented here plays out on two levels: between therapeutic repertoires and within them. Therapeutic repertoires are the result of deliberate (and recurring) strategies of value work: The normative uptake of claims of plasticity is coordinated by concern-making and by raising promises. The kind of value work advocates were able to do, what kind of problems and solutions were articulated and propagated, depended largely on how those advocates distributed agency and determinism amongst the individual subject, her brain, and the environment - whichever is needed to cater to different action programmes and moral positions.

Within repertoires, a recurring pattern can be seen in how actors, in different phases of value work, attributed agency to different actors. In each repertoire, concerns of specific audiences had to be addressed, articulated, and translated into brain problems (diagnoses), before the plastic brain could be proposed as 
(part of) a solution. It is through concern-making that specific audiences are appealed to and persuaded to care for neuroscience knowledge. Actors linked a variety of concerns to the brain: from troublesome teenagers, who 'confused' parents and made them 'insecure' (chapter two); to the anxiety of stress, the fear of productivity loss, and a lack of job satisfaction (chapter three); and on to seniors' fear of cognitive decline (chapter four). These central concerns, once identified, were translated into a brain problem: the teenage brain, "out of balance" due to its susceptibility to environmental stimuli; the evolutionary-grown rigid brains of stressed employees; and the neural deterioration or reorganization of the ageing brain. It is through this form of value work that neuro-advocates reiterate 'negative' or undesirable cultural stereotypes by describing them in terms of the brain. As we have seen, such understandings of teenagers, employees, and seniors thereby become normalized: They are aimed at taking away the individual burden of responsibility or guilt for such behaviours by redirecting them to the brain. As a result, the plastic brain in these kinds of repertoires did not change that we still expect teenagers to be troublesome, employees to be stressed out, and seniors to be cognitively slow.

In this form of value work, the agency for change (who is changing what) becomes located outside of the individual subject and is attributed either to the brain itself and/or to social or cultural structures in the environment which would imprint the brain. Every diagnosis in the repertoires across the three cases concerns a mismatch between the individual subject on one side, and her brain and/or the environment on the other side. In this mismatch, it is the individual's lack of agency that is presented as an obstacle to flourishing, which effectively casts individuals as passive recipients and victims of their own brains and/or the environment.

Only when a concern is translated into a problem of the brain can a solution in terms of the brain be promised. In this second, promissory stage of value work, agency is returned to the individual subject. In every repertoire, the individual subject is given the capability and responsibility to influence her brain for the better. Parents (as substitutes for the agency of teenagers) are encouraged to coordinate the stimuli that imprint their adolescent's brains. Employees are promised that by practicing mindfulness as brain training, they can overcome the evolutionary grown rigidity in their stressed brains. And seniors are told that they either should exercise and thereby delay the deterioration of their brains or cherish the unique qualities that this reorganization brings. 
Just as undesirable stereotypes became described in neuro-terms, so too more positive or desirable behaviours became reiterated and normalized as an innate potential of the plastic brain: normality, creativity, sociality, and idealism of teenagers; efficiency, productivity, kindness, compassion, and well-being of workers; and cognitive health, emotional stability, and wisdom of seniors. It is by promising that ideals are already dormant in the plastic brain - that some moral destabilizations in self-understandings occurred: Making desirable behaviour an intrinsic quality of our plastic brain functioned as a biological resistance against negative or dominant cultural stereotypes. For example, Crone's plea for siding with teenagers by stressing their unique qualities and debunking parents' negative view of them, or the emergence of the wise ageing brain to address the societal position of seniors and debunk concerns about cognitive decline propagated through brain games.

What parents, employees, and seniors are allowed to hope for is often articulated in terms of the brain. A normal brain for teenagers, a productive or happy brain for employees, and a young or wise brain for seniors. The goal of practicing the brain does not seem to be behavioural change, but brain change. The effects of practicing and thereby changing your brain are in all cases presented as being of a higher order than changes in or through behaviour. It is implicit in all repertoires that a change in brain structure is more solid, more fixed, more sustainable than a changed mind or changed behaviour. The ideal of a changed brain entails that a changed brain makes it easier for the individual subject to automatically do and be good. Thus, in a final move, we see that agency flows back from the individual subject to the brain itself. The dance of agency is complete. ${ }^{60}$

The ambiguity of plasticity also played out between therapeutic repertoires. On this level, we see how the question of who should change what is answered differently. In each repertoire, the individual subject is granted the agency to overcome the mismatch between the individual subject, her brain, and the environment. However, it is not only the individual and her brain that are encouraged to change. In every case, a repertoire emerged in which the environment is a substantial part of the solution. In the case of the teenage brain, the environment is not only valued as harmful but also as a necessary resource for teenage brains to acquire experiences necessary for self-actualization. In the case of the

${ }^{60}$ The dance of agency between brain, individual subject, and the environment in therapeutic repertoires of a plastic brain bears a close resemblance to the dance of agency that occurs in practices of neurofeedback, as vividly described by Brenninkmeijer (2013). 
mindful brain, the ideal employee is not only productive and flexible, but also kind, empathic, and able to contribute to a collegial and inviting company culture. In the case of the ageing brain, seniors should not only try to stave off cognitive decline; they also deserve an environment that cherishes their unique, wise brains. While substantial social critiques based on a plastic brain did not occur in the cases studies, the environment did often play a role as a (problematic) condition that should be improved in order for a particular plastic brain to flourish.

While in each case the individual is encouraged to change her brain in order to adapt to the challenges of the environment, repertoires diverge in their assessment of whether the environment should also be expected to adapt to the plastic brain. This explains the bifurcation of the repertoires in each case. Combined with the dance of agency within repertoires - the movement from brain to individual subject to brain - this indicates that the plastic brain in practice does not appear to be strong enough to break through familiar dualistic reasoning, characteristic for agency/structure and nature/nurture debates. It is through the plastic brain that both individual and environment are addressed as both objects and agents of change. It is through the plastic brain that both stability and change are hoped for.

\section{A Brain Inspired Good Life: virtues of change}

In all cases studied here, knowledge of the plastic brain is mobilized as part of a solution for a particular challenge, implying a specific view on the society or world in which we should flourish. Despite the promise of a final answer to such challenges, the multiple repertoires show that advocates use the plastic brain to respond differently to societal challenges. What emerges from the challenges for which the plastic brain is mobilized as a solution is that a braininspired good life needs virtues that are directed towards coping with and cultivating (societal) change.

In chapter two, we saw that the ideal adolescent was one with a normal teenage brain, whereby normality came to mean both the absence of abnormal behaviour or mental disorders and the adherence to norms of societal success. Parents seemed to struggle to prepare their adolescents for successfully entering a complex, meritocratic society, where abnormal behaviour increasingly becomes medicalized, digital technologies challenge education, and social relations increasingly are maintained online. In chapter three, the ideal employee that emerged was an emotionally controlled employee, cognitively productive 
and efficient, who contributes to a satisfying company culture by being happy, compassionate, kind, and empathic. To flourish, and to cope with new demands at the workplace, employees needed to combine emotional self-control with social self-fashioning. The environment in which this employee flourishes is a fast-paced, flexible, and precarious work economy. In chapter four, the ideal senior is cognitively healthy, which could mean either still cognitively performing effectively or enjoying the fruits of wisdom. This ideal senior presupposes a hypercognitive society in which maintaining cognitive health in older age becomes a mark of ageing well, and where wisdom is celebrated for its contribution to a dynamic knowledge society.

These societal developments and challenges are often cast as dynamic, permanently changing, and beyond our control. In such a society, where (social, political, economic) changes are presented as permanent, complex, and inevitable, the locus of change shifts towards the individual. As we have seen throughout the cases, plasticity became valued in terms of change: the becoming of the teenage brain, the resilience and self-transformation of the mindful brain, and the adaptation and (again) resilience of the ageing brain. These valuations exemplify that the individual has the ability to harmonize herself with the permanent changes in society. Through a plastic brain, advocates present virtues aimed at either coping with or internalizing change. Ideals such as normality (in the sense of brain development), self-regulation, and resilience represent a world-to-mind direction of fit: They are about coping with undesirable challenges. In contrast, virtues such as flexibility, creativity, sociality, and wisdom have a mind-to-world direction of fit: They are about internalizing and adapting to change. Both types of virtues emerge as ways to help the individual come to terms with a lack of control and grip (cf. McMahon, 2005; Swierstra, 2016). By doing so, the individual is addressed as having an ethical task for improving her life.

Plasticity as the cultivation of change emerges as a modern-day virtue: a character disposition that is desirable because it helps an individual lead a good life and because it is deemed desirable by the outside world (Swierstra, 2016). If we want to become better parents, more productive and happy employees, or more resilient and youthful seniors, we have to address ourselves as ethical agents of our own lives - and practice our brains. Plasticity thus is not so much about satisfying certain needs - as the neo-liberal explanation would have it but about practicing and learning to turn yourself into a person who acts virtuously. Learning a virtue, according to Aristotle, Epicurus, and other virtue ethi- 
cists, takes effort and can only be practiced through external examples. Throughout the cases, we have seen some of these external examples, such as a parent-as-external-frontal-lobe, a mindfulness coach, or descriptions of exercising and wise seniors.

In contrast to the pessimists' treatment of plasticity as a purely individualistic form of neuro-enhancement or self-care, we found that the plastic brain in practice was also made valuable for a multiplicity of non-individualistic, relational, or social-oriented values and virtues. For example, as we have seen, the plastic brain is used to counteract negative stereotypes of both teenagers and seniors, aiming to rehabilitate their societal position. Similarly, in the case of the mindful brain, employees are encouraged through mindfulness as brain training to become better colleagues and to help improve their company culture. What the emergence of such therapeutic repertoires shows is that a neurobiological self-fashioning of the individual does not rule out the environment: It is for the social good and the well-being of others that we are urged to have a kind of reflexive understanding about the workings of our brains. Neurobiological self-fashioning should therefore not be seen as simply individualistic or asocial, as others have also rightly pointed out (Abi-Rached \& Rose, 2013; Nagel \& O'Connor, 2017; Pickersgill, 2013).

The concern of many critics of the ethical impact of the neurosciences on society, such as Pitts-Taylor (2008), Thornton (2011), Abi-Rached \& Rose (2013), and many others, is that what starts as a focus on self-fashioning as a way to cope with a lack of control will turn into a societal obligation. There is a genuine risk that can becomes ought (a Kantian reversal). Practicing your plastic brain to improve yourself then becomes another choice and individual responsibility in a neo-liberal meritocracy or knowledge economy, where not being able or willing to do so becomes blameworthy, and failure becomes your own fault. After having studied the plastic brain in three societal domains, I only partly share this critique.

What such critiques tend to overlook is that appealing to individuals as ethical agents of their own lives in the face of societal challenges is not the same as making them responsible for these challenges. Conflating the two under the label "neo-liberal" seems to obscure rather than to clarify. The question is not whether the plastic brain empowers or disciplines our behaviour, but rather: When does addressing individuals as ethical agents of their own lives become too demanding? Addressing parents as ethical agents responsible for the normal development of their adolescent's brains (in whatever meaning) seems 
more acceptable than addressing employees to take care of their own stressful work conditions. But under what conditions, and how, does something desirable - such as individual ethical agency - become an obstacle to flourishing?

To answer this question, a more substantial view on the social ills of society would be needed. It's here that I share some of the worry of the pessimists. In contrast to classic virtue ethics accounts of Aristotle, Epicurus, and others, repertoires of plasticity do not always come with a substantial critique of societal developments. While some repertoires critically address social and economic developments in the environment in which we are supposed to flourish - such as the medicalization of abnormal behaviour in the case of the teenage brain, the fast pace of working in the case of the mindful brain, or health care policy in the case of the ageing brain - such developments are mostly presented as too complex to fall within our reach. True to the historical tradition of ethical advice of virtue ethicists, the individual is instead encouraged in plasticity discourse to change its inner world (i.e., brain). While such appeals to individuals as ethical agents of their own lives are applaudable, without a substantial critique on society, the solution can only treat half of the problem at best.

And not all problems get articulated in plasticity discourse. The story that is not being told using plasticity arguments involve action programmes that deal with fate, tragedy, or acceptance, where an individual has to make peace with her unjust nature or destiny. One could imagine using plasticity to argue that parenting teenage kids is downright impossible, as their impulsive and emotional brains are just too unpredictable to control. Similarly, using the mindful brain to argue that contemporary work conditions make it impossible for our primitive brains to work well is another story not being told. While such logically conceivable examples may sound exaggerated, the lack of tragedy and fate becomes more apparent in the case of the ageing brain, where plasticity of the ageing brain is not being used to prepare seniors for the fourth age - a period of illness, suffering, existential dread, and death. The plastic brain is limited by hope. Fate, tragedy, and the acceptance of injustice are not part of its valuations in practice.

\section{Conclusion}

What does the case of the plastic brain tell us about the ways we should study and ethically assess the societal impact of the neurosciences? As we have seen 
in the introduction, there are roughly two positions in the debate on the societal impact of the neurosciences. Enthusiasts celebrate the plastic brain as empowering and liberating from structural constraints, while pessimists fear a reiteration of the status quo - which, in a context of neo-liberal bio-medicalization, would imply a further constraining or disciplining of our options to act and care for ourselves. Both positions ascribe a specific societal performativity to brain claims, a performativity that is narrowly limited to valuations that emphasize either empowerment or control.

Both enthusiasts and pessimists seem to imply that disseminators, appropriators, and users are merely passive recipients of such knowledge. This assumption is unwarranted. A neuroscience understanding of behaviour doesn't speak for itself: Technical brain facts are made valuable when they are used, and as such receive the potential to influence or destabilize our norms, values, and ideas of the good. Facts and values become easily intertwined in contexts where brain claims are mobilized to answer questions of what to do and how to live. This observation has some consequences for exploring the ethical impact of neuroscience in society.

What the multiple cases in this thesis have shown is that moral changes (such as the promise to achieve happiness more easily, or the fear for an increased individual responsibilization) cannot be predicted or assumed on a general, theoretical level. Valuations of the plastic brain do not automatically flow from the lab, nor are they the inevitable result of ideological superstructures in society. As we have seen in the previous chapters, the possible ethical meaning and relevance of a plastic brain are the outcome of deliberate, practical, and contextual processes of valuation. By explicating this value work, I have shown that neuro-advocates and appropriators have a certain autonomy in the ways they make brain claims meaningful, relevant, and valuable for specific audiences in specific contexts. Ignoring these deliberate efforts of advocates to make brain claims valuable can result in overlooking the multiplicity and entanglements of brain claims and values in practice.

Theoretical frameworks that are centred on the question of empowerment versus control, and a priori give one the upper hand, lack in explanatory force for studying the impact of technoscience on society. As we have seen, such valuations can co-exist happily together in practice, as they are the result of deliberate value work. Whether and under what conditions addressing the ethical agency of an individual results in moralizing or over-asking should be a subject of explicit empirical scrutiny in specific cases. We have to take the effort to em- 
pirically question how possible destabilizations are constructed in practice, and how multiple valuations are made to co-exist or clash together, as we cannot assume beforehand that neuro-induced moral changes will or will not occur.

With this thesis, I have shown how the plastic brain is normatively used to address problems and ideals across our entire lifespan. My selection of cases and my focus on discursive material have some obvious limitations. Other plastic brains (e.g., brains of infants or stroke patients), and other contexts (e.g., education or mental care), could result in other types of repertoires. Other valuations of the plastic brain might occur when observing parents, employees, or seniors in their daily doings, or when studying such valuations in other (nonWestern, non-affluent) countries. Each case in this dissertation could be followed up by extensive ethnographic fieldwork to show the messiness of certain value work and valuations in practice - a fruitful venue for further study.

I have also shown that popular understandings of neuroscience ("neurohype") are not only about scientific credibility or authority. Instead of dismissing popular usages of the neurosciences as hype, bad, or over-inflated interpretations of science, neurohype can be a rich resource for understanding public ethical deliberation. As we have seen, the potential of the plastic brain foremost shows in its normative versatility to be used in society as ethics by other means: It allows advocates to mobilize and combine different concerns, challenges, action programmes, and ideals. Compared to the notion of interpretative flexibility of facts and artefacts (Pinch \& Bijker, 1984), so well-known in Science and Technology Studies, the case of the plastic brain implies that neuroscience knowledge claims do not gain force through closure mechanisms but rather through being used simultaneously for different normative ends. The notion of value work thereby not only demonstrates the role values and valuations play in the stabilization of scientific knowledge in society but also demonstrates the reverse - the role scientific claims play in the (de)stabilization of our moral order. As such, I hope to have contributed to the risen interest in the field of STS towards explicating the deliberate and tacit activities of actors in keeping both the social, material, and moral order stable (cf. Bowker \& Star, 1999; Denis, Mongili, \& Pontile, 2015; Dussauge et al., 2015; Puig de la Bellacasa, 2011).

\section{Valuing Techno-Science in Times of Moral Need}

It is often said that in our contemporary, pluralistic, liberal societies, the question of how to lead the good life is banned from the public domain (Swierstra, 2002; Waelbers and Briggle, 2010). When we look at the ways the plastic brain 
emerged in our popular culture, it seems that the question of how to live well seems as topical as ever - albeit it comes dressed in scientific guise. In a society where continuous (technological) change is the imperative, reflection on what to do and how to live forces itself upon us in our daily lives. With this thesis, I hope to have given a glimpse of how we came to use the sciences of the brain to articulate challenges and ideals of the good life in our contemporary "therapy culture" (cf. Brinkman, 2016; Furedi, 2004; Illouz, 2008; McGee, 2005).

I aimed to develop a way of tracing valuations and normativities of brain facts in popular culture by introducing the concepts of value work and therapeutic repertoire. Both concepts help to make visible how science popularisers and translators (often scientists themselves) have to engage with values in order to make their claims valuable, and how this results in recurring patterns of moral argumentation about these claims. Explicating the ways actors can engage with values shows the hidden labour that is necessary to make brain claims relevant and usable. Science is not made relevant in a void: The valuation of science in society necessarily reflects cultural traditions, challenges and ideals. An insight that cannot be repeated often enough in our times of factchecking and post-truth.

Neuroscientists acknowledge more and more often that they find themselves in the challenging situation in which they have to juggle between fulfilling strong promises and meeting expectations to secure funding and attract media attention on the one hand, and downplaying the scope and power of the discipline by stressing the complexities and uncertainties of research on the other (Abi-Rached \& Rose, 2013). The perverse effects of publication pressure and funding schemes in our academic landscape notwithstanding, I do not think that neuroscientists (or other scientists for that matter) in practice should refrain from trying to make their research relevant and valuable for specific ends or audiences. To make such a plea would be hopelessly naïve. In our times of 'post-normal science' (Ravetz, 1999), where science faces political and public pressures for valorisation, valuation efforts have become part and parcel of being a good academic. If neuroscientists would indeed resist the urge for translation, this would effectively black box the possible impacts of their own knowledge claims. This seems rather unhelpful for the debate on the possible impact of emerging science and technologies, such as the neurosciences. Understanding how facts and values necessarily are intertwined when translating or popularizing scientific knowledge can strengthen the ethical sensibility of neu- 
roscientists (and other popularisers) to the possible societal and normative effects of their work.

As the neurosciences continue to mature as a scientific field, the public debate about its possible impact on our lives will continue to grow. For humanities scholars and ethicists, or any other scholar interested in the ethical implications of new science and technologies, this means that we have a clear task before us. Understanding and explicating how values and valuations are part of the translation of science to wider audiences might make it easier to direct the focus of our critique: both towards the implicit moral labour of scientists and science journalists as well as towards our own willingness to accept certain moral stories over others. When science and technologies promise us a better life, we - scholars, translators, citizens - must address ourselves as ethical agents, and explicitly question what doing and being good should mean in our present society. 



\section{LIST OF FIGURES}

Figure 1: Model of a therapeutic repertoire of living well showing the relations amongst its four key variables.

Figure 2: Eveline Crone's two bestselling popular-science books on the teenage brain, which established the notion of the teenage brain in public discourse in the Netherlands.

Figure 3. Therapeutic Repertoire I: Parents as guardians of external stimuli. In this repertoire, parents work to protect the normal brain development of their adolescent through prohibition, punishment, and reward.

Figure 4. Therapeutic repertoire II: Parents as supportive coaches. In this repertoire, parents are urged to encourage the self-actualization of their adolescents, by motivating teenagers to develop their unique character traits.

Figure 5: The French genetic scientist and Buddhist monk Matthieu Ricard (left), and Richard Davidson (right). Ricard, one of the co-authors and participants in Davidson \& Lutz's study (2008), became widely portrayed in popular media as "the happiest man in the world," as his test-score in the study was an outlier (Bates, 2012). Photo credit: Jeff Miller, WisconsininNews, June 2008.

Figure 6. Therapeutic repertoire I: the mindful brain as cognitive enhancer. In this repertoire, employees are encouraged to practice mindfulness in order to increase the productivity, efficiency and resilience of their plastic brains.

Figure 7. Therapeutic repertoire II: the mindful brain as soft skill. In this repertoire, employees are encouraged to practice mindfulness to become more compassionate, kind, creative and happy in the workplace. 
Figure 8: Trap des Ouderdoms, anoniem, $1640-1660$. Source:

Rijksmuseum.

Figure 9. First therapeutic repertoire of an ageing brain. In this repertoire, seniors are encouraged to prevent and delay cognitive decline, and maintain their youthful cognitive performance, by training their cognitive reserve through physical exercises.

Figure 10. Second therapeutic repertoire of an ageing brain. In this repertoire, seniors are encouraged to stay socially engaged, not worry too much, and embrace their unique, wise brains. 


\section{REFERENCES}

Abi-Rached, J. M., \& Rose, N. (2013). Neuro: The new brain sciences and the management of the mind. Princeton: Princeton University Press.

"A consensus on the brain training industry from the scientific community [open letter]." (2014, October 20). Max Planck Institute for Human Development and Stanford Center on Longevity. Retrieved from http://longevity3.stanford.edu/blog/2014/10/15/the-consensus-on-the-braintraining-industry-from-the-scientific-community/

Adam, D. (2005, July 27). Plan for Dalai Lama lecture angers neuroscientists. The Guardian. Retrieved: http://www.theguardian.com/world/2005/jul/27/research. highereducation.

Aleman, A. (2012). Het Seniorenbrein. Amsterdam: Uitgeverij Atlas Contact.

Aleman, A. (2014). Our ageing brain: How our mental capacities develop as we grow older. London, England: Scribe.

Alidina, A., \& Adams, J. (2014). Mindfulness at work for dummies. West-Sussex: Wiley.

Annas, J. (1993). The morality of happiness. New York, NY: Oxford University Press.

Anthony, P. D. (1977). The ideology of work. Great Britain: Tavistock.

Appiah, A. (2008). Experiments in ethics. Cambridge, MA: Harvard University Press.

Ariès, M. (1962). Centuries of childhood: A social history of family life. New York, NY: Alfred A. Knopf.

Arnett, J. J. (2006). G. Stanley Hall's Adolescence: Brilliance and nonsense. In: History of Psychology, 9(3), 186-197.

Arnett, J. J. (2013). Adolescence and emerging adulthood: A cultural approach. New Jersey: Pearson.

Bakker, J. H. (2008, March 13). De dingen die we (niet) vergeten. Algemeen Dagblad, p. 14.

Barbrook, R. (2006). The class of the new. London, England: OpenMute.

Bates, C. (2012, October 31). Is this the world's happiest man? Brain scans reveal French monk has 'abnormally large capacity' for joy - thanks to meditation. Retrieved from http://www.dailymail.co.uk/ health/article-2225634/Is-worlds-happiest-man-Brain-scans-reveal-French-monk-abnormallylarge-capacity-joy-meditation.html

Baumrind, D. (1971). Current patterns of parental authority. Developmental Psychology Monographs, 4(1.2), 1.

Baumrind, D. (1991). Effective parenting during the early adolescent transition. In P. A. Cowan \& E. M. Hetherington (Eds.), Advances in family research (Vol. 2). Hillsdale, NJ: Erlbaum.

Beaulieu, A. (2000). The Space inside the skull: Digital representations, brain mapping and cognitive neuroscience in the Decade of the Brain. (Doctoral dissertation). Amsterdam: University of Amsterdam.

Begley, S. (2009). The plastic mind. New York, NY: Ballantine Books. 
Bessant, J. (2008). Hard wired for risk: Neurological science, "the adolescent brain" and developmental theory. Journal of Youth Studies, 11(3), 347-360.

Bhattacharjee Y. (2005, November 18). Neuroscientists welcome Dalai Lama with mostly open arms. Science, 310(5751), 1104.

Bijsterveld, K. T. (1996). Geen kwestie van leeftijd: Verzorgingsstaat, wetenschap en discussies rond ouderen in Nederland, 1945-1982. Amsterdam: Van Gennep.

Binstock, R. H. (2005). Old-age policies, politics, and ageism. Generations, 26(3), 73-78.

Birren, J. E., \& Bengtson, V. L. (1988). Emergent theories of aging. New York, NY: Springer Publishing Company.

Blakemore, S. J. (2008). The social brain in adolescence. Nature Reviews Neuroscience, 9(4), 267.

Bloemink, S. (2011, December 14). De plooibaarheid van het puberbrein. Retrieved from https://www.groene.nl/artikel/in-de-watten-leggen-die-jongeren

Boltanski, L., \& Chiapello, E. (2005). The new spirit of capitalism. London, England: Verso.

Borup, M., Brown, N., Konrad, K., \& Van Lente, H. (2006). the sociology of expectations in science and technology. Technology Analysis \& Strategic Management, 18(3/4), 285-298.

Bowker, G. C., \& Star, S. L. (1999). Sorting things out: Classification and its consequences. Cambridge, MA: MIT Press.

Brandt, E. (2011, March 26). Beweeg voor je brein (het is nooit te laat om te beginnen). Trouw, p. 2.

Brendel, D. (2015, February 11). There are risks to mindfulness at work. Retrieved from https://hbr.org/2015/02/there-are-risks-to-mindfulness-at-work

Brinkgreve, C. (2012). Het verlangen naar gezag: Over vrijheid, gelijkheid en verlies van houvast. Amsterdam: Atlas Contact.

Brinkman, S. (2016). Standvastig. Onder Alle Omstandigheden Jezelf Blijven. Amsterdam: Lev AW Bruna.

Brenninkmeijer, J.M. (2013). Brain Technologies of the self: How working on the self by working on the brain constitutes a new way of being oneself (Doctoral dissertation). Groningen: Rijksuniversiteit Groningen.

Brod, C. (1984). Technostress: The Human Cost of the Computer Revolution. Reading, U.K.: AddisonWesley.

Brown, N. (2003). Hope against hype - Accountability in biopasts, presents and futures. Science Studies, 16(2), 3-21.

Butler, R. N. (1969). Ageism: Another form of bigotry. The Gerontologist, 9(4), 243-246.

Cabeza, R. (2002). Hemispheric asymmetry reduction in old adults: The HAROLD model. Psychology and Aging, 17, 85-100.

Carey, B. (2005, October 19). Scientists Bridle at Lecture Plan for Dalai Lama. The New York Times. Retrieved: http://www.nytimes.com/2005/10/19/national/19meditate. html?pagewanted=all\&_r=0

Carr, D. C., \& Komp, K. (2011). Gerontology in the Era of the Third Age: Implications and next steps. New York, NY: Springer.

Casey, B. J., Jones, R. M., \& Hare, T. A. (2008). The adolescent brain. Annals of the New York Academy of Sciences, 1124, 111-126.

Cassidy, A. (2006). Evolutionary psychology as public science and boundary work. Public Understanding of Science, 15(2), 175-205.

Caufield, T. et al. (2010). "Neurohype" and the name game: Who's to blame? AJOB: Neuroscience, 1(2), 13-14.

Cederström, C., \& Spicer, A. (2015). The wellness syndrome. Cambridge, U.K.: Polity Press. 
Center for Advanced Studies in Business. (2017, October 18). Cultivating Well-being in the Workplace. Retrieved from Wisconsin School of Business website: http://uwcultivatingwellbeing.com/ program-details/

Chaskalson, M. (2011). The mindful workplace: Developing resilient individuals and resonant organizations with MBSR. West-Sussex, England: Wiley-Blackwell.

Choudhury, S. (2010). Culturing the adolescent brain: What can neuroscience learn from anthropology? Social Cognitive Affective Neuroscience, 5(2-3), 159-167.

Choudhury, S., \& Slaby, J. (2012). Critical neuroscience: A handbook of the social and cultural contexts of neuroscience. Chinchester, West Sussex: Wiley-Blackwell.

CogniFit. (2016a). Neuroplasticity and cognition structure and organization. Retrieved October 12, 2016, from https://www.cognifit.com/brain-plasticity-and-cognition

CogniFit. (2016b). Memory loss: Things your brain should know about itself. Retrieved October 12, 2016, from https://www.cognifit.com/memory-loss

CogniFit. (2016c). Mild Cognitive Impairment (MCI) Neurodegenerative disease: Help prevent cognitive deterioration. Retrieved October 12, 2016, from https://www.cognifit.com/mild-cognitiveimpairment

Cole, T. R. (1992). The journey of life: A cultural history of aging in America. New York, NY: Cambridge University Press.

Cooter, R. (1984). The cultural meaning of popular science: Phrenology and the organization of consent in Nineteenth-Century Britain. Cambridge, U.K.: Cambridge University Press.

Costa, L., \& Veloso, A. (2015). The gamer's soul never dies: Review of digital games for an active ageing [conference paper]. DOI: 10.1109/CISTI.2015.7170614. Retrieved from https://www.researchgate.net/ publication/282850880_The_gamer\%27s_soul_never_dies_Review_of_digital_games_for_an_ac tive_ageing

Cozolino, L. (2008). The healthy aging brain: Sustaining attachment, attaining wisdom. New York, NY: W. W. Norton.

Creswell, J. D., Pacilio, L.E., Lindsay, E.K., \& Brown, K.W. (2014). Brief mindfulness meditation training alters psychological and neuroendocrine responses to social evaluative stress. Psychoneuroendocrinology 44, 1-12.

Crone, E. A. (2007, June 12). Interview by G. Jochems [radio interview]. Noorderlicht Radio.

Crone, E. A. (2008). Het Puberende Brein. Amsterdam: Bert Bakker.

Crone, E. A. (2012). Het Sociale Brein van de Puber. Amsterdam: Bert Bakker.

Crone, E. A., \& Dahl, R. E. (2012). Understanding adolescence as a period of social-affective engagement and goal flexibility. Nature Reviews Neuroscience, 13(9), 636-650.

Crone, E. A. (2013, April). De puzzelstukjes vallen juist steeds beter op hun plek. De Psycholoog, 1718.

Crone, E. A. (2017). The adolescent brain. New York, NY: Routledge.

Dalai Lama. (2005). Science at the crossroads [talk given at the annual meeting of the Society for Neuroscience on November 12, 2005]. Retrieved from http://www.dalailama.com/messages/bud dhism/science-at-the-crossroads

Davidson, R. J., Kabat-Zinn, J., Schumacher, J., Rosenkranz, M., Muller, D., Santorelli, S. F., ... \& Sheridan, J. F. (2003). Alterations in brain and immune function produced by mindfulness meditation. Psychosom Med., 65(4), 564-570.

Davidson, R. J., \& Lutz, A. (2008). Buddha's brain: Neuroplasticity and meditation. IEEE Signal Process Mag., 25(1), 176-174. 
Davidson, R. J. (2012). The emotional life of your brain: How its unique patterns affect the way you think, feel, and live - and how you can change them. New York, NY: Penguin.

Davidson, R. J. (2015, October 26). How science can inform good leadership. HuffingtonPost. Retrieved from https://www.huffingtonpost.com/richard-j-davidson/how-science-can-informgo_b_8347478.html

Davies, W. (2015). The happiness industry: How the government and big business sold us well-being. London, England: Verso.

Davis, S. W., Dennis, N.A., Daselaar, S.M., Fleck, M.S. \& Cabeza, R. (2008). Que PASA?: The posterior-anterior shift in aging. Cerebral Cortex, 18(5), 1201-1209.

Degryse, I. (2013, January 9). Als je trager denkt, neem je betere beslissingen. Knack, p. 68.

De Haan, B. (2006, July 1). Game puzzles: Brain training (Nintendo DS). Elsevier, p. 91.

Dehue, T. (2008). De Depressie-epidemie. Amsterdam: Augustus.

Dehue, T. (2014). Betere Mensen: Over gezondheid als keuze en koopwaar. Amsterdam: Atlas Contact.

De Jongh, R., \& Ridsdale, C. (2010, February 1). Het leven van je brein. Psychologie Magazine, 45.

De Lange, B. (2012). Breinbewust Leven: Dement worden? Vergeet het maar? Amsterdam: Uitgeverij SWP.

De Lange, F. (2013). Imagining good ageing. In M. Schermer \& W. Pinxten (Eds.), Ethics, health policy and (anti-) aging: Mixed blessings (pp. 135-147). Dordrecht: Springer.

De Lege, J. (2007, September 22). Hoe oud is jouw brein? De Volkskrant, p. 5.

Den Blijker, J. (2012, March 23). Alles wat ouders moeten weten over drinkende pubers. Trouw, p. 8.

Denis, J., Mongili, A., \& Pontille, D. (2015). Maintenance \& repair in science and technology studies. TECNOSCIENZA, 6(2), 5-15.

Depaepe, M., Simon, F., Van Gorp, A. (2005). Paradoxen van pedagogisering. Handboek pedagogische historiografie. Leuven/Voorburg: Acco.

De Vries, H. (2012, October 20). De kracht van een bejaard brein. Reformatorisch Dagblad, p. 19.

Dewey, John (1913). The problem of values. Journal of Philosophy, Psychology and Scientific Methods, 10(10), 268-269.

Dewey, J. (1939). Theory of valuation. International Encyclopedia of Unified Science, 2(4), 1-67.

Dewey, J. (1954). The later works. Athens, OH: Swallow Press.

De Winter, M. (2005). Oratie: Democratieopvoeding versus de code van de straat. Utrecht: Universiteit Utrecht.

Dewulf, D. (2014). Heartful Leven, Mindful Werken. Tielt: Lannoo.

Dillaway, H. E., \& Byrnes, M. (2009). Reconsidering successful aging: A call for renewed and expanded academic critiques and conceptualizations. Journal of Applied Gerontology, 28(6), 702722 .

Dohmen, J., \& Baars, J. (Eds). (2010). De kunst van het ouder worden: De grote filosofen over ouderdom. Amsterdam: Ambo/Antos.

Dor-Ziderman, Y., Berkovich-Ohana, A., Glicksohn, J., \& Goldstein, A. (2013). Mindfulness-induced selflessness: a MEG neurophenomenological study. Fronties in human neuroscience, 7, 582.

Drucker, P. F. (1993) [1946]. Concept of the corporation. London, England: Transaction.

Dugomay, P., \& Mok, D. (2009). Een kijkje onder de zinderende hersenpan van adolescenten: Hand-out bij de workshop Het Puberende Brein. Amstelveen: Herman Wesselink College Amsterdam.

Dumit, J. (2004). Picturing personhood: Brain scans and biomedical identity. Princeton: Princeton University Press.

Dussauge, I., Helgesson, C., Lee, F. (2015). Value practices in the life sciences and medicine. Oxford, U.K.: Oxford University Press. 
Dussauge, I., Helgesson, C., Lee, F., \& Woolgar, S. (2015). On the omnipresence, diversity, and elusiveness of values in the life sciences and medicine. In I. Dussauge, et al., Value Practices in the Life Sciences and Medicine. Oxford, U.K.: Oxford University Press.

Eerkens, M. (2007). Wat gebeurt er toch allemaal in dat koppie? J/M, 10, $22-25$.

Egher, C., \& Wyatt, S. (2016). Testing memory, shifting responsibility: Internet-based (self) diagnostics of Alzheimer's disease. In M. Boenink, H. van Lente, E. Moors (Eds.), Emerging technologies for diagnosing Alzheimer's disease (pp. 141-163). London, England: Palgrave/Macmillan.

Ehmer, J., \& Lis, C. (2016). The idea of work in Europe from antiquity to modern times. New York, NY: Routledge.

Evenblij, M. (2003, October 25). Slijtage aan het hersenspoor; Alzheimer Publieksdag over ouder worden en vergeten. De Volkskrant, p. 1G.

Fairclough, N. (1995). Critical discourse analysis: The critical study of language. New York, NY: Longman.

Fahnestock, J. (1998) [1986]. Accommodating science: The rhetorical life of scientific facts. Written Communication 15(3), 330-350.

Farb, N.A., Segal, Z.V., \& Anderson, A.K. (2013). Mindfulness meditation training alters cortical representations of interoceptive attention. Social Cognitive and Affective Neuroscience, 8(1), 15-26.

Federal Trade Commission. (2016a). Lumosity to pay \$2 million to settle FTC deceptive advertising charges for its "Brain Training" program [press release]. Retrieved October 12, 2016, from https://www.ftc.gov/news-events/press-releases/2016/01/lumosity-pay-2-million-settle-ftcdeceptive-advertising-charges

Federal Trade Commission. (2016b). Complaint against Lumos Labs. Retrieved from https://www.ftc.gov/system/files/documents/cases/160105lumoslabscmpt.pdf

Florida, R. L. (2002). The rise of the creative class: And how it's transforming work, leisure, community and everyday life. New York, NY: Basic.

Foute afstemming puberhersenen (2008, September), Mediaplanet Themabijlage Onze Hersenen, p. 5.

Fries, J. F. (2002). Aging, natural death, and the compression of morbidity. Bulletin of the World Health Organization, 80(3), 245-250.

Friswell, J. H. (1976). Michel de Montaigne essays (Vol. 1). London, England: Gordon.

Furedi, F. (2004). Therapy culture: Cultivating vulnerability in an uncertain age. New York, NY: Routledge.

Ganguly, K., \& Poo, M. (2013). Activity-dependent neural plasticity: From bench to bedside. Neuron, 80(3), 729-741.

Gard, T., Hölzel, B. K., Sack, A. T., Hempel, H., Lazar, S. W., Vaitl, D., \& Ott, U. (2011). Pain attenuation through mindfulness is associated with decreased cognitive control and increased sensory processing in the brain. Cerebral Cortex, 22(11), 2692-2702.

Gelles, D. (2015, January 21). Amid the chattering of the global elite, a silent interlude. The New York Times. Retrieved from https://dealbook.nytimes.com/2015/01/21/amid-the-chattering-of-theglobal-elite-a-silent-interlude/

George, D. R., \& Whitehouse, P. J. (2011). Marketplace of memory: What the brain fitness industry says about us and how we can do better. Gerontologist, 51(5), 590-596.

Gerritsen, W. (2014, February 25). Hoe meer je weet, hoe meer je vergeet. De Stentor, p. 3.

Giddens, A. (1991). Modernity and self-identity. London, England: Polity Press.

Giedd, J. N., Blumenthal, J., Jeffries, N. O., Castellanos, F. X., Liu, H., Zijdenbos . . \& \& Rapoport, J. L. (1999). Brain development during childhood and adolescence: A longitudinal MRI study. $\mathrm{Na}-$ ture Neuroscience, 2(10), 861-863. 
Gilleard, C., \& Higgs, P. (2002). The Third Age: Class, cohort or generation? Ageing and Society, 22(3), 369-82.

Goldberg, E. (2006). The wisdom paradox: How your mind can grow stronger as your brain grows older. New York, NY: Penguin.

Goldin, P. R., \& Gross, J. J. (2010). Effects of mindfulness-based stress reduction (MBSR) on emotion regulation in social anxiety disorder. Emotion 10(1), 83-91.

Goleman, D. (1995). Emotional intelligence: Why it can matter more than IQ. New York, NY: Bantam.

Goleman, D.P. (1995). Emotional intelligence: Why it can matter more than IQ for character, health and lifelong achievement. New York: Bantam Books.

Goleman, D.P. (2003). Healing emotions: Conversations with the Dalai Lama on mindfulness, emotions, and health. Boston: Shambhala.

Goleman, D.P. (2013a, December). The focused leader. Harvard Business Review, 52-60. Retrieved from https://hbr.org/2013/12/the-focused-leader

Goleman, D.P. (2013b). Focus: The hidden driver of excellence. London, England: HarperCollins.

GoogleTechTalks (2009, September 28). Transform your mind, change your brain: Neuroplasticity and personal transformation by Richard J. Davidson [video file]. Retrieved from http://www.youtube.com/watch?v=7tRdDqXgsJ0\&NR=1

Goslinga, R., \& Spiering, H. (2012, June 23). Als puber leefde ik bij de dag, dat mis ik wel. NRC Handelsblad, pp. 16-17.

Graeber, D. (2001). Toward an anthropological theory of value: The false coin of our own dreams. New York, NY: Palgrave.

Graeber, D. R. (2013, August 20). On the phenomenon of bullshit jobs. Libcom. Retrieved from https://libcom.org/library/phenomenon-bullshit-jobs-david-graeber

Grant, J. A., Courtemanche, J., Duerden, E. G., Duncan, G. H., \& Rainville, P. (2010). Cortical thickness and pain sensitivity in zen meditators. Emotion 10(1), 43-53.

Grubbs, L. (2016). The arts and sciences of reading: Humanities in the laboratory. AJOB Neuroscience, $7(2), 85-94$.

Hagner, M. (2009). The mind at work. The visual representation of cerebral processes. In R. van de Vall \& R. Zwijnenberg (Eds.), The body within: Art, medicine and visualisation. Leiden: Brill.

Haijtema, A. (2012, October 30). Interview: Neuropsycholoog Andre Aleman. De Volkskrant, 2.

Hajer, M. A. (2006). Doing discourse analysis: Coalitions, practices, meaning. In M. Van den Brink \& T. Metze (Eds.), Word matter in policy and planning: Discourse theory and method in the social sciences. Utrecht: NETHUR.

Hall, G. S. (1904). Adolescence: Its psychology and its relations to physiology, anthropology, sociology, sex, crime, religion and education (Vol. 2). New York, NY: Appleton.

Halffman, W., \& Radder, H. (2015). The academic manifesto: From an occupied to a public university. Minerva, 53(2), 165-187.

Hamburger, O., \& Bergsma, A. (2013). Happiness at work: Improve your self-leadership skills to flourish at work. Amsterdam: Boom/Nelissen.

Hamer, H. (2012, June 6). “Eerst skater, nu punker: Laat die puber toch.” Trouw, pp. 8-9.

HAPPYneuron. (2016). Efficiency of Brain Training. Retrieved October 12, 2016, from http://www.happy- neuron.com/brain-and-training/why-train-your-brain

Harris, J. (1998). The nurture assumption: Why children turn out the way they do. New York, NY: Simon \& Schuster.

Heinemann, T. (2012). Populäre Wissenschaft. Hirnforschung zwischen Labor und Talkshow (Popular science: Brain research between laboratory and pop culture). Göttingen: Wallstein. 
Heller, A. (1988). General ethics. Oxford: Basil Blackwell Ltd.

Helgesson, C. F., \& Muniesa, F. (2013). For what it's worth: An introduction to valuation studies. Valuation Studies, 1(1), 1-10.

Hertogh, C. (2013). Aging beyond frailty: The future of old age. In M. Schermer \& W. Pinxten (Eds.). Ethics, health policy and (anti-)aging: Mixed blessings (pp. 91-105). Dordrecht: Springer.

Hildt, E., \& Leefmann, J. (Eds). (2017). The human sciences after the Decade of the Brain. London, England: Elsevier.

Hill, R. B. (1996). Historical context of the work ethic. Athens: University of Georgia.

Hoe werkt een puber? (2012, June 5). Het Parool, p. 6.

Hofmeester, K., \& Moll-Murata, C. (2011). The joy and pain of work: Global attitudes and valuations, 1500-1650 [Introduction]. International Review of Social History, 56(S19), 1.

Holstein, B., Parks, J. A., \& Waymack, M. H. (2011). Ethics, aging and society: The critical turn. New York, NY: Springer.

Hölzel, B.K., Carmody, J., Evans, K.C., Hoge, E.A., Dusek, J.A., Morgan, L., ... \& Lazar, S.W. (2010). Stress reduction correlates with structural changes in the amygdala. Social Cognitive and Affective Neuroscience, 5(1), 11-17.

Hölzel, B.K., Carmody, J., Vangel, M., Congleton, C., Yerramsetti, S.M., Gard, T., \& Lazar, S.W. (2011). Mindfulness practice leads to increases in regional brain gray matter density. Psychiatry Research, 191(1), 36-43.

Horst, M. (2016). Reframing science communication: Culture, identity, and organisations. London, England: Department of Science and Technology Studies, UCL.

Horsthuis, A. (2008). Lach, luister, voel mee en wees helder. J/M Puberspecial, 2, 11-15.

Horwitz, A. V., \& Wakefield, J. C. (2007). The loss of sadness. How psychiatry transformed normal sorrow into depressive disorder. Cary, NC: Oxford University Press.

Horwitz, A. V. (2016). What's normal? Reconciling biology and culture. New York, NY: Oxford University Press.

Hougaard, R., Carter, J., \& Coutts, G. (2016). One second ahead: Enhance your performance at work with mindfulness. New York, NY: Palgrave Macmillan.

Houtekamer, C. (2010, May 15). Pubers zijn eigenlijk hele leuke mensen: Interview Hersenonderzoeker Eveline Crone. NRC Handelsblad, p. 6.

Hume, D., Norton, D. F., \& Norton, M. J. (2000)[1739]. A treatise of human nature. Oxford: Oxford University Press.

Ihde, D. (1990). Technology and the lifeworld. Bloomington, IN: Indiana University Press.

Illes, J., Moser, M.A., McCormick, J.B., Racine, E., Blakeslee, S., Caplan, A., ... \& Nicholson, C. (2010). Science and society: Neurotalk: Improving the communication of neuroscience research. Nature Reviews Neuroscience, 11, 61-69.

Illouz, V. (2008). Saving the modern soul: Therapy, emotions, and the culture. Berkeley, CA: University of California Press.

Jaarsma, P., \& Welin, S. (2012). Autism as a natural human variation: Reflections on the claims of the neurodiversity movement. Health Care Analysis, 20(1), 20-30.

Jansen Schoonhoven, M. (2013, January 31). Beste ouders: Eis van uw kind gehoorzaamheid. Trouw, p. 21.

Jensen, F. E., \& Nutt, A. E. (2015). The teenage brain: A neuroscientist's survival guide to raising adolescents and young adults. New York, NY: HarperCollins.

Jolles, J. (2011, December 10). Puberen? Verbreinen! Het Parool, p. 16. 
Jordan-Young, R., \& Rumiati, R. (2012). Hardwired for sexism? Approaches to sex/gender in neuroscience. Neuroethics, 5(3), 305-315.

Johnson, J., \& Littlefield, M., eds. (2012). The neuroscientific turn: Transdisciplinarity in the Age of the Brain. Ann Arbor: University of Michigan Press.

Josipovic, Z. (2014). Neural correlates of nondual awareness in meditation. Annals of the New York Academy of Sciences 1307(1), 9-18.

Kabat-Zinn J. (1982). An outpatient program in behavioral medicine for chronic pain patients based on the practice of mindfulness meditation: Theoretical considerations and preliminary results. General Hospital Psychiatry, 4(1), 33-47.

Kabat-Zinn J. (1990). Full catastrophe living: Using the wisdom of your body and mind to face stress, pain, and illness. New York, NY: Dell.

Kabat-Zinn, J. (2001). Mindfulness meditation for everyday life. London, England: Piatkus.

Kabat-Zinn J. (2003). Mindfulness-based interventions in context: Past, present, and future. Clinical Psychology: Science and Practice, 10(2), 144-56.

Kahn, R. (2011). De Tien Geboden voor het Brein [Ten commandments for the brain]. Amsterdam: Balans.

Kahneman, D. (2011). Thinking fast and slow. New York, NY: Farrar, Straus and Giroux.

Katz, S. (2013). Active and successful aging: Lifestyle as a gerontological idea. Recherches sociologiques et anthropologiques, 44(44-1), 33-49.

Katz, S., \& Peters, K. R. (2008). Enhancing the mind? Memory medicine, dementia, and the aging brain. Journal of Aging Studies, 22(4), 348-355.

Kawashima, R. (2005). Train your brain: 60 days to a better brain. Teaneck (NJ): Kumon Publishing.

Keulartz, J. Schermer, M., Korthals, M., \& Swierstra, T. (2004). Ethics in Technological Culture. Science, Technology \& Human Values, 29(1), 3-29.

Kjellberg, H., \& Mallard, A. (2013). Valuation studies? Our collective two cents. Valuation Studies, $1(1), 11-30$.

Koenig, R. (2014, October 22). Brain-training companies get advice from some academics, criticism from others. The Chronicle. Retrieved from http://www.chronicle.com/article/Brain-TrainingCompanies-Get/149555

Koole, W. (2013). Mindful Werken. Amsterdam: Business Contact.

Koops, W. (2013, April 19). Over de Kleren van de Keizer Neuro. De Psycholoog.

Korteweg, N. (2010, April 3). Billenkoek voor het brein. NRC Handelsblad.

Kraus, C. (2012). Critical studies of the sexed brain: A critique of what and for whom? Neuroethics, 5(3), 247-259.

Kueider, A. M., Parisi, J. M., Gross, A. L., \& Rebok, G. W. (2012). Computerized cognitive training with older adults: A systematic review. PLOS One, 7(7), e40588.

Kuiper, S. (2008, March 24). Interview met Douwe Draaisma. De Groene Amsterdammer.

Lamont, Michèle. 2012. Towards a comparative sociology of valuation and evaluation. Annual Review of Sociology 38, 201-221.

Lampit, A., Hallock, H., \& Valenzuela, M. (2014). Computerized cognitive training in cognitively healthy older adults: A systematic review and meta-analysis of effect modifiers. PLoS medicine, 11(11), e1001756.

Land, D. (2008). Study shows compassion meditation changes the brain [news article]. University of Wisconsin-Madison. Retrieved from http://news.wisc.edu/study-shows-compassion-meditationchanges-the-brain/

Laslett, P. (1987). The emergence of the third age. Ageing \& Society, 7(2), 133-160. 
Laslett, P. (1991). A fresh map of life: The emergence of the Third Age. Cambridge, MA: Harvard University Press.

Latour, B. (1992). Where are the missing masses? The sociology of a few mundane artifacts. In W. E. Bijker and J. Law (Eds.), Shaping technology/building society: Studies in sociotechnical change (pp. 225-259). Cambridge, MA: MIT Press.

Latour, B. (2004). Why has critique run out of steam? From matters of fact to matters of concern. Critical Inquiry, 30(2), 225-248.

Latour, B. (2005). Reassembling the social: An introduction to actor-network-theory. New York, NY: Oxford University Press.

Lawless, M., \& Augoustinos, M. (2017). Brain health advice in the news: Managing notions of individual responsibility in media discourse on cognitive decline and dementia. Qualitative Research in Psychology, 14(1), 62-80.

Lazar, S. W., Kerr, C.E., Wasserman, R.H., Gray, J.R., Greve, D.N., Treadway, M.T., ... \& Rauch, S.L. (2005). Meditation experience is associated with increased cortical thickness. Neuroreport, 16(17), 1893

Ledoux, J. (2002). The synaptic self: How our brains become who we are. New York, NY: Viking Penguin.

Lemke, T. (2011). Biopolitics: An advanced introduction. New York, NY: New York University Press.

Lesko, N. (2001). Act your age! A cultural construction of adolescence. New York, NY: Routledge.

Lindahl, J. R., Fisher, N. E., Cooper, D. J., Rosen, R. K., \& Britton, W. (2017). The varieties of contemplative experience: A mixed-methods study of meditation-related challenges in Western Buddhists, PLOS One, 12(5), 1-38.

Littlefield M. M., \& Johnson J. M. (Eds.) (2012). Introduction: Theorizing the neuroscientific turn: Critical perspective on a translational discipline. In M. Littlefield \& J. M. Johnson (Eds.), The neuroscientific turn. Transdisciplinarity in the Age of the Brain. Ann Arbor: University of Michigan Press.

Lopez, D.S. (2008). Buddhism and science: A guide for the perplexed. Chicago: University of Chicago Press.

Luciana, M. (2011). Development of the adolescent brain: Neuroethical implications for the assessment of executive functions. In B. J. Sahakian \& J. Illes (Eds.), Oxford handbook of neuroethics. Oxford, U.K.: Oxford University Press.

Lucivero, F., Swierstra, T., \& Boenink, M. (2011). Assessing expectations: Towards a toolbox for an ethics of emerging technologies. NanoEthics, 5(2), 129.

Lutz, A., Greischar, L.L., Rawlings, N.B., Ricard, M., \& Davidson, R.J. (2004). Long-term meditators self-induce high-amplitude gamma synchrony during mental practice. PNAS, 101(46), 1636916373.

Lutz, A., Dunne, J. D., \& Davidson, R. J. (2007). Meditation and the neuroscience of consciousness: An introduction. In P. D. Zelazo, M. Moscovitch, \& E. Thompson (Eds.), Cambridge handbook of consciousness (pp. 499-551). Cambridge, U.K.: Cambridge University Press.

Lutz, A., Brefczynski-Lewis, J., Johnstone, T., \& Davidson, R.J. (2008). Regulation of the neural circuitry of emotion by compassion meditation: Effects of meditative expertise. PLOS One, 3(3), 1897.

Maastricht University. (2017, June 1). Constructing Worlds: Reflections on Science, Technology and Democracy - Wiebe Bijker [Video file]. Retrieved from https://www.youtube.com/watch?v= Frt0UnTjS_A. 
Maccoby, E. E., \& Martin, J. A. (1983). Socialization in the context of the family: Parent-child interaction. In P. H. Mussen (Ed.) \& E. M. Hetherington (Vol. Ed.), Handbook of child psychology: Socialization, personality, and social development (Vol. 4). New York, NY: Wiley.

MacIntyre, A. (1985). After Virtue: A Study in Moral Theory. London: Duckworth.

MacLean, Paul D. (1990). The triune brain in evolution: Role in paleocerebral functions. New York, NY: Plenum.

Mahncke, H. (2016, October 3). Brain training and its critics [Web log post]. Retrieved October 12, 2016, from http://blog.brainhq.com/2016/10/03/brain-training-critics/

Malabou, C. (2008). What should we do with our brain? New York, NY: Fordham University Press.

Males, M. (2009). Does the adolescent brain make risk taking inevitable?: A skeptical appraisal. Journal of Adolescent Research, 24(1), 3-20.

Marieke. (2012, November 14). Ik worstel, maar kom nog niet boven [forum post]. Retrieved from https://www.ouders.nl/forum/ouders-en-school/ik-worstel-maar-kom-nog-niet-boven

Martinez, A. G., \& Mendoza-Denton, R. (2011). The prospect of plasticity: Malleability views of group differences and their implications for intellectual achievement. Mental/Behavioral Health, and Public Policy, 5(1), 137-159.

Martinson, M., \& Berridge, C. (2015). Successful aging and its discontents: A systematic review of the social gerontology literature. The Gerontologist, 55(1), 58-69.

Max Geheugentrainer. (2016). Retrieved October 12, 2016, from http://www.omroepmax.nl/max geheugentrainer/

McGee, M. (2005). Self Help Inc.: Makeover culture in American life. New York, NY: Oxford University Press.

McGrath, R. (2014, July 30). Management's three eras: A brief history. Harvard Business Review. Retrieved from https://hbr.org/2014/07/managements-three-eras-a-brief-history

McKinley, W., \& Mone, M. A. (2003). Micro and macro perspectives in organization theory: A tale of incommensurability. In H. Tsoukas \& C. Knudsen (Eds.). The Oxford handbook of organization theory. Oxford, U.K.: Oxford University Press.

Melby-Lervåg, M., \& Hulme, C. (2013). Is working memory training effective? A meta-analytic review. Developmental Psychology, 49(2), 270-291.

Mellor, F. (2003). Between fact and fiction: Demarcating science from non-science in popular physics books. Social Studies of Science 33(4), 509-538.

Merzenich, K. (2010, October 27). Giving health advice for older people? Don't forget the brain! [Web log post]. Retrieved from http://blog.brainhq.com/2010/10/27/giving-health-advice-for-olderpeople-dont-forget-the-brain/

Merzenich, M. (2013). Soft-wired: How the new science of brain plasticity can change your life. San Francisco: Parnassus.

Merzenich, M. (2014). Cognitive Training Data Response Letter (open letter to the Stanford Center on Longevity). Retrieved October 12, 2016, from Cognitive Training Data website: http://www.cognitivetrainingdata.org/the-controversy-does-brain-training-work/responseletter/

Mesman, J. (2008). Uncertainty in medical innovation: Experienced pioneers in neonatal care [Technology and Society Series]. Health. Hampshire, U.K.: Palgrave MacMillan.

Millington, B. (2012). Use it or lose it: Ageing and the politics of brain training. Leisure Studies, 31(4), 429-446.

“Mindfulness werkt voor de ondernemer." (n.d.). Retrieved April 26, 2016, from Dokter Bosman website: https://dokterbosman.nl/mindfulness-werkt-ondernemer/ 
Ministerie van Sociale Zaken en Werkgelegenheid. (2014, May 13). Vierjarige aanpak van beroepsrisico nummer 1: Werkstress [news item]. Retrieved from https://www.rijksoverheid.nl/actueel/ nieuws/2014/05/13/vierjarige-aanpak-van-beroepsrisico-nummer-1-werkstress

Mohr, W. K. (2003). Discarding ideology: The nature/nurture endgame. Perspectives in Psychiatric Care, 39, 113-121.

Mol, A. (2002). The body multiple: Ontology in medical practice Durham, NC: Duke University Press.

Mudde, T. (2013, April 6). Muziek voor de geest. De Volkskrant.

Muniesa, F. (2011). A flank movement in the understanding of valuation. The Sociological Review, $59(2), 24-38$

Mykytyn, C.E. (2010). A history of the future: The emergence of contemporary anti-ageing medicine. Sociology of Health \& Illness, 32(2), 181-196.

Nelis, H. \& Van Sark, Y. (2012). Puberbrein Binnenstebuiten. Utrecht: Kosmos Uitgevers.

Neugarten, B. (1974). Age groups in American society and the rise of the young-old. The Annals of the American Academy of Political and Social Science, 415(1), 187-198.

Nintendo. (2006). Brain Age [Nintendo DS game].

Nintendo. (2016). Top selling software sales units. Retrieved October 12, 2016, from https://www.nintendo.co.jp/ir/en/sales/software/ds.html

Nussbaum, M. (1994). The therapy of desire: Theory and practice in Hellenistic ethics. Princeton: Princeton University Press.

NWO. (2017, June 16). "NWO-Spinozapremies voor Eveline Crone, Albert Heck, Michel Orrit en Alexander van Oudenaarden." Retrieved from https://www.nwo.nl/actueel/nieuws/2017/nwospinozapremies-voor-eveline-crone-albert-heck-michel-orrit-en-alexander-vanoudenaarden.html

O'Connor, C., Rees, G., \& Joffe, H. (2012). Neuroscience in the public sphere. Neuron, 74(2), 220-226.

O'Connor, C., \& Joffe, H. (2013). How has neuroscience affected lay understandings of personhood? A review of the evidence. Public Understanding of Science, 22(3), 254-268.

O'Connor, C., \& Nagel, S. K. (2017). Neuro-enhancement practices across the lifecourse: Exploring the roles of relationality and individualism. Frontiers in Sociology, 2, 1.

Oden, E., \& Hald, S. (2008, September 1). Hoe ouder het brein, hoe beter. Psychologie Magazine, 88.

Older, D. (2014). Beyond Bullshitting: valorisation and the humanities in the Netherlands [master thesis]. Maastricht: Maastricht University.

Orme, N. (2003). Medieval children. New Haven: Yale University Press.

Ortega, F. (2009). The cerebral subject and the challenge of neurodiversity. BioSocieties, 4(4): 425-445.

Ortega, F. (2011). Toward a genealogy of neuroascesis. In F. Ortega \& F. Vidal (Eds.) Neurocultures: Glimpses into an expanding universe. Frankfurt-am-Main, Germany: Lang.

Ortega, F., \& Vidal, F. (Eds.) (2011). Neurocultures: Glimpses into an expanding universe. Frankfurt-amMain: Lang.

Pagnoni, G., \& Cekic, M. (2007). Age effects on gray matter volume and attentional performance in Zen meditation. Neurobiology of aging, 28(10), 1623-1627.

Palmore, E. B. (1999). Ageism: Negative and positive. New York, NY: Springer.

Papadopoulos, D. (2011). The imaginary of plasticity: Neural embodiment, epigenetics and ectomorphs. The Sociological Review, 59(3), 432-456.

Pardoen, J. (2008, October/November). Pubers kunnen niet anders dan puberen. Leef! Magazine, 810.

Pek, A., \& Van Osnabrugge, S. (2013, November 1). Oud worden zonder bijwerkingen: 35 manieren om gezonder te leven. Pyschologie Magazine, p. 35. 
Pickering, A. (1995). The mangle of practice: Time, agency, and science. Chicago: University of Chicago Press.

Pickersgill, M., \& Van Keulen, I. (Eds.). (2011). Sociological reflections on the neurosciences (Vol. 13). Bingley, U.K.: Emerald Group.

Pickersgill, M. (2011). Connecting neuroscience and law: Anticipatory discourse and the role of sociotechnical imaginaries. New Genetics and Society, 30(1), 27-40.

Pickersgill, M. (2013). The social life of the brain: Neuroscience in society. Current Sociology, 61(3), 322-340.

Pickersgill, M., Broer, T., Cunningham-Burley, S., \& Deary, I. (2017). Prudence, pleasure, and cognitive ageing: Configurations of the uses and users of brain training games within U.K. media, 2005-2015. Social Science \& Medicine, 187, 93-100.

Pickut, B. A., Van Hecke, W., Kerckhofs, E., Mariën, P., Vanneste, S., Cras, P., \& Parizel, P. M. (2013). Mindfulness based intervention in Parkinson's disease leads to structural brain changes on MRI: A randomized controlled longitudinal trial. Clinical Neurology and Neurosurgery, 115(12), 2419-2425.

Pinch, T. J., \& Bijker, W. E. (1984). The social construction of facts and artefacts: Or how the sociology of science and the sociology of technology might benefit each other. Social Studies of Science, 14(3), 399-441.

Pitts-Taylor, V. (2010). The plastic brain: Neoliberalism and the neuronal self. Health, 14(6), 635-652.

Pitts-Taylor, V. (2016). The brain's body: Neuroscience and corporeal politics. Durham: Duke University Press.

PLUS Brein Trainer. (2016). Veroudering bestrijden. Retrieved October 12, 2016, from http://breintrainer.plusonline.nl/waarom-oefenen/veroudering-bestrijden.html

Pols, J. (2004). Good care. Enacting a complex ideal in long-term psychiatry. Utrecht: Trimbos Instituut.

Pont, S. (2010, February 4). Untitled. Het Parool, p. 3.

Popma, J. (2012). Techno-stress; Verkenning van een risico in opkomst Onderzoek in opdracht van de vakcentrale FNV en Hugo Sinzheimer Instituut. Amsterdam: Universiteit van Amsterdam.

Posit Science. (2016a). Memory lapses. Retrieved October 12, 2016, from http://www.brainhq.com/ brain-resources/memory/memory-lapses

Posit Science. (2016b). Breaking news on brain training and dementia. Retrieved October 12, 2016, from http://www.brainhq.com/active-study-results

Post, S.G. (2000). The concept of Alzheimer disease in a hypercognitive society. In P. J. Whitehouse, K. Maurer, \& J. F. Ballenger (Eds.), Concepts of Alzheimer disease: Biological, clinical, and cultural perspectives. Baltimore: Johns Hopkins University Press.

Pronk, I. (2013, January 25). Ouders van nu willen geen gehoorzaam kind. Trouw, p. 3-4.

Ptries. (2013, March 25). Eindelijk begrijp ik ook mijzelf...! [book review comment]. Retrieved from https://www.bol.com/nl/p/het-puberendebrein/1001004005997720/?suggestionType=typedsearch

Puig de la Bellacasa, M. (2011). Matters of care in technoscience: Assembling neglected things. Social Studies of Science, 41(1), 85-106.

Purser, P., \& Loy, D. (2013, July 15t). Beyond McMindfulness. HuffingtonPost. Retrieved from http://www.huffingtonpost.com/ron-purser/beyond-mcmindfulness_b_3519289.html

Purser, P. (2016, March 22). Cutting through the corporate mindfulness hype. HuffingtonPost. Retrieved from http://www.huffingtonpost.com/ron-purser/cutting-through-the-corporatemindfulness-hype_b_9512998.html

Putnam, H. (2002). The collapse of the fact/value dichotomy and other essays. Harvard University Press. 
Quart, A. (2012, November 23). Neuroscience: Under attack. The New York Times. Retrieved from http://www.nytimes.com

Quine, W.V. (1951). Main Trends in Recent Philosophy: Two Dogmas of Empiricism. The Philosophical Review, 60(1), 20-43.

Racine E. (2010). Pragmatic neuroethics. Cambridge, MA.: MIT Press.

Ramscar, M., Hendrix, P., Shaoul, C., Milin, P., \& Baayen, H. (2014). The myth of cognitive decline: Nonlinear dynamics of lifelong learning. Topics in Cognitive Science, 6(1), 5-42.

Ravetz, J. (1999.) What is post-normal science? Futures, 31, 647-653.

Rees, T. (2010). Being neurologically human today: Life and science and adult cerebral plasticity (An ethical analysis). American Ethnologist, 37(1), 150-166.

Rees, T. (2016). Plastic reason. An anthropology of brain science in embryogenetic terms. Oakland: University of California Press.

Ritsema, B. (2012, June 1). De synapsen, spines, gliacellen, astrocyten van ouderen. NRC Handelsblad.

Rock, D. (2009). Your brain at work: Strategies for overcoming distraction, regaining focus, and working smarter all day long. New York, NY: HarperCollins.

Rose, S. (1997). Lifelines: Biology, freedom, determinism. London, England: Penguin.

Rose, N. (2003). Neurochemical selves. Society, 41(1), 46-59.

Rose, S. (2005). The future of the brain. The promise and perils of tomorrow's neuroscience. Oxford, U.K.: Open University Press.

Rose, N. (2007). The politics of life itself: Biomedicine, power, subjectivity in the Twenty-First Century. Princeton: Princeton University.

Roskies, A. (2002). Neuroethics for the new millenium. Neuron, 35(1), 21-23.

Rotman, B. (2000). Going parallel. SubStance, 29(1), 56-79.

Rowe, J. W., \& Kahn, R. L. (1987). Human aging: Usual and successful. Science, 237(4811), 143-149.

Rowe, J. W., \& Kahn, R. L. (1997). Successful aging. The Gerontologist, 37(4), 433-440.

Rozendaal, S. (2012, May 5). Sporten maakt slim. Elsevier, p. 62.

Rubin, B. P. (2009). Changing brains: The emergence of the field of adult neurogenesis. Biosocieties, $4(4), 407-424$.

Ryberg, J. (2014). Punishing adolescents: On immaturity and diminished responsibility. Neuroethics, $7(3), 327-336$.

Sandel, M. J. (2007). The case against perfection: Ethics in the age of genetic engineering. Cambridge, MA: Harvard University Press.

Sayer, A. (2011). Why things matter to people: Social science, values and ethical life. Cambridge, U.K.: Cambridge University Press.

Schatzki, T. R., Cetina, K., \& Von Savigny, E. (2001). The practice turn in contemporary theory. London, England: Routledge.

Schirmann, F. (2013). Invoking the brain in studying morality: A theoretical and historical perspective on the neuroscience of morality. Theory \& Psychology, 23(3), 289-304.

Schmitz, S., \& Höppner, G. (2014). Neurofeminism and feminist neurosciences: a critical review of contemporary brain research. Frontiers in human neuroscience, 8, 546.

Schneider, T., \& Woolgar, S. (2015). Neuroscience beyond the laboratory: Neuro knowledges, technologies and markets. BioSocieties, 10(4), 389-399.

Schwartz, J. M., \& Begley, S. (2002). The mind and the brain: Neuroplasticity and the power of mental force. New York, NY: HarperCollins. 
Seligman, M. (2002). Authentic happiness: Using the new positive psychology to realize your potential for lasting fulfillment. New York, NY: Free Press.

Sennett, R. (1999). The corrosion of character: The personal consequences of work in the new capitalism. New York, NY: W. W. Norton.

Shapin, S. (2009). The scientific life: A moral history of a late modern vocation. Chicago: University of Chicago Press.

SharpBrains. (2013). The digital brain health market, 2012-2020: Web-based, mobile and biometrics-based technology to assess, monitor and enhance cognition and brain functioning. San Francisco, CA: SharpBrains.

SharpBrains. (2015). The digital brain health market 2012-2020: Web-based, mobile and biometrics-based technology to assess, monitor, and enhance cognition and brain functioning [Addendum]. San Francisco, CA: SharpBrains.

Shaw, C. A., \& McEachern, J. C. (Eds.). (2001). Toward a theory of neuroplasticity. Hove: Psychology Press.

Siegel, D. J. (2007a). The mindful brain: Reflection and attunement in the cultivation of well-being. New York, NY: W. W. Norton.

Siegel, D. J. (2007b). Mindfulness training and neural integration: Differentiation of distinct streams of awareness and the cultivation of well-being. Social Cognitive and Affective Neuroscience, 2(4), 259-263.

Siegel, D. J. (2010). Mindsight: The new science of personal transformation. New York, NY: Bantam.

Simons, D. J., Boot, W.R., Charness, N., Gathercole, S.E., Chabris, C.F., Hambrick, D.Z., \& StineMorrow, E.A.D.J. (2016). Do "brain-training" programs work? Psychological Science in the Public Interest, 17(3), 103-186.

Sitskoorn, M. (2008). Lang Leven de Hersenen. Amsterdam: Uitgeverij Bert Bakker.

Slote, M. (2001). Morals from motives. Oxford, U.K.: Oxford University Press.

Smith, A. (1776) [1976]. An inquiry into the nature and causes of the Wealth of Nations. Chicago: University of Chicago Press.

Society for Neuroscience. (2007, June). Brain briefings: Adult neurogenesis. Retrieved April 26, 2017, from http://www.tutis.ca/NeuroMD/L3AssMem/olfactory.pdf

Spacks, P. M. (1982). The adolescent idea: Myths of youth and the adult imagination. London, England: Faber \& Faber.

Spinoza, B. (1997). On the improvement of the understanding (R. H. M. Elwes, Trans. [1995]. New York, NY: Dover), sec. 17:2. Project Gutenberg. Retrieved from http://www.gutenberg.org/files/1016/1016-h/1016-h.htm (Original work published 1662)

Spock, B. (1946). The common sense book of baby and child care. New York, NY: Duell, Sloan, and Pearce.

Steenhorst, R. (2014, October, 29). Digitale breintraining is tijdverspilling [Digital Brain Training is a Waste of Time]. Telegraaf.

Strauch, B. (2003). The primal teen: What the new discoveries about the teenage brain tell us about our kids. New York, NY: Anchor.

Sun, J. (2014). Mindfulness in context: A historical discourse analysis. Contemporary Buddhism, 15(2), 394-415.

Swierstra, T. (2002). Moral vocabularies and public debate. The cases of cloning and new reproductive technologies. In J. Keulartz, M. Korthals, M. Schermer, \& T. Swierstra (Eds), Pragmatist ethics for a technological culture. Deventer: Kluwer Academic. 
Swierstra, T., \& Rip, A. (2007). Nano-ethics as NEST-ethics: Patterns of moral argumentation about new and emerging science and technology. Nanoethics, 1(1), 3-20.

Swierstra, T., Van Est, R., \& Boenink, M. (2009). Taking care of the symbolic order. How converging technologies challenge our concepts. Nanoethics, 3(3), 269-280.

Swierstra, T. (2011). Heracliteïsche ethiek. Omgaan met de soft impacts van technologie batesInaugural lecture]. Maastricht, the Netherlands: Maastricht University.

Swierstra, T. (2015). Identifying the normative challenges posed by technology's 'soft' impacts. Etikk i praksis. Nordic Journal of Applied Ethics, 9(1), 5-20.

“TV Kijkcijfers." Retrieved October 12, 2016, from https://kijkonderzoek.nl/component/ com_kijkcijfers/Itemid,133/file,dp-10-1-0-p

Tallis, R. (2009). Neurotrash. New Humanist, 124(6). Retrieved from http://newhumanist.org.uk/ 2172/neurotrash

Tan, C. M. (2012). Search inside yourself: The unexpected path to achieving success, happiness (and world peace). New York, NY: HarperCollins.

Tang, Y. Y., Hölzel, B. K., \& Posner, M. I. (2015). The neuroscience of mindfulness meditation. Nature Reviews Neuroscience, 16(4), 213-225.

Tang, Y.Y., Ma, Y., Wang, J., Fan, Y., Feng, S., Lu, Q., ... \& Posner, M.I. (2007). Short-term meditation training improves attention and self-regulation. Proceedings of the National Academy of Sciences USA 104(43), 17152-17156.

Tang, Y.Y., Lu, Q., Geng, X., Stein, E.A., Yang, Y., \& Posner, M. (2010). Short-term meditation induces white matter changes in the anterior cingulate. Proceedings of the National Academy of Sciences USA, 107(35), 15649-15652.

Tang, Y.Y., ., Tang, R., Jiang, C., \& Posner, M.I. (2014). Short-term meditation intervention improves self-regulation and academic performance. Journal of Child Adolescent Behavior, 2(4), 251.

Taylor, C. (1986). Sources of the self: The making of the modern identity. Cambridge, MA: Harvard University Press.

Teeven, F. (2012). Wijziging van het Wetboek van Strafrecht, het Wetboek van Strafvordering en enige andere wetten in verband met de invoering van een adolescentenstrafrecht [memorie van toelichting]. Retrieved from http://www.eerstekamer.nl/behandeling/20121205/memorie_van_toelichting/ document3/f=/vj5dn4f0nwuz.pdf

Teskey, G.C. (2001). A general framework for neuroplasticity theories and models. In C.A. Shaw \& J.C. McEachern (Eds.), Toward a theory of neuroplasticity (pp. 6-10). Philadelphia, PA: Taylor and Francis.

Thornton, D.J. (2011). Brain Culture: Neuroscience and Popular Media. New Brunswick, NJ: Rutgers University Press.

Tippett, K. (Host). (2012, December 27). Jon Kabat-Zinn: Opening to Our Lives [radio program]. Retrieved from https://onbeing.org/programs/jon-kabat-zinn-opening-to-our-lives/

Toulmin, S. (1958). The Uses of Argument. Cambridge: Cambridge University Press.

Tresch, J. (2011). Experimental ethics and the science of the meditating brain. In F. Ortega \& F. Vidal (Eds.), NeuroCultures: Glimpses in an expanding universe (pp. 49-68). Frankfurt am Main: Peter Lang.

Turney, J. (2008). Popular science books. In M. Bucchi \& B. Trench (Eds.), Handbook of public communication of science and technology (pp. 5-14). London, England: Routledge.

Trevarthen, C. (1987). Plasticity in the nervous system. In R. L. Gregory (Ed.), The Oxford companion to the mind. Oxford, U.K.: Oxford University Press. 
Väänänen, A., Anttila, E., Turtiainen, J., \& Varje, P. (2012). Formulation of work stress in 1960-2000: Analysis of scientific works from the perspective of historical sociology. Social Science $\mathcal{E}$ Medicine, 75(5), 784-794.

"Vakbonden willen meditatie in cao." (2008, September 3). Trouw. Retrieved from https:/www.trouw.nl/home/vakbonden-willen-meditatie-in-cao a027ec09/

Van Beek, M. (2012, June 28). Liefdesverdriet bedwingen met Boeddha en de Tao. Trouw, p. 6.

Van Dam, N. T., van Vugt, M. K., Vago, D. R., Schmalzl, L., Saron, C. D., Olendzki, A. . . \& \& Meyer, D. E. (2017). Mind the hype: A critical evaluation and prescriptive agenda for research on mindfulness and meditation. Perspectives on Psychological Science, 13(1), 36-61.

Van de Werff, T., Slatman, J., \& Swierstra, T. (2016). Can we "Remedy" neurohype, and should we? Using neurohype for ethical deliberation. AJOB Neuroscience, 7(2), 97-99.

Van de Werff, T. (2017). Ethics by neurological means: How the teenage brain is used to talk about good parenting. In E. Hildt \& J. Leefmann (Eds.), The human sciences after the Decade of the Brain. London, England: Elsevier.

Van den Brink, G., Van Hulst, M., Maalsté, N., Peeters, R., \& Soeparman, S. (2012). Hoe Dionysos in Haren verscheen: Maatschappelijke facetten van Project $X$ Haren. Tilburg: Tilburgse School voor Politiek en Bestuur.

Van der Heijden, M. (2013, April 13). Ruzie over het Puberbrein. NRC Handelsblad.

Van der Neut, D. (2007, July 1). Het kneedbare brein. Psychologie Magazine.

Van der Roest, P. (2013). Mindful Leidinggeven. Haarlem: Altamira.

Van der Sman, J. (2006, August 12). Welzijn: hoe word je 100? Elsevier, p. 66-71.

Van der Zee, E. (2012). Het Vitale Brein: Hoe de hersenen sterk en dynamisch te houden. Amsterdam: Bert Bakker.

Van Hintum, M. (2009, February 14). Vergeet het geheugen \& breintraining: Wetenschappers vinden dat mensen zich te druk maken over normale vergeetachtigheid. De Volkskrant, p 1.

Van Hintum, M. (2012, September 15). Het idee van de 'rijpende' hersenen is toch wat simplistisch. De Volkskrant, p. 6.

Van Lente, H. (2012). Navigating foresight in a sea of expectations: Lessons from the sociology of expectations. Technology Analysis \& Strategic Management, 24(8), 769-782.

Van Luijk, F. (2011). Waarom Werken Wij? De betekenis van werken 1983-2008/2009 [dissertation]. Amsterdam: Vrije Universiteit Amsterdam.

Verbeek, P. P. (2000). De daadkracht der dingen - over techniek, filosofie en vormgeving. Amsterdam: Boom.

Vermeulen, M. (2015, September 19). Waarom zou dit nu net onoplosbaar zijn? De Volkskrant.

Vestergaard-Poulsen, P., van Beek, M., Skewes, J., Bjarkam, C. R., Stubberup, M., Bertelsen, J., \& Roepstorff, A. (2009). Long-term meditation is associated with increased gray matter density in the brain stem. Neuroreport, 20(2), 170-174.

Vidal, C. (2012). The sexed brain: Between science and ideology. Neuroethics, 5(3), 295-303.

Wainwright, S. P., Williams, C., Michael, M., Farsides, B., \& Cribb, A. (2006). Ethical boundary-work in the embryonic stem cell laboratory. Sociology of Health $\mathcal{E}$ Illness, 28(6), 732-748.

Waelbers, K., \& Briggle, A. (2010). Technology, the good life, and liberalism: Some reflections on two principles of neutrality. Techné: Research in Philosophy and Technology, 14(3), 176-193.

Wax, R. (2013). Sane new world: Taming the mind. London, England: Hodder \& Stoughton.

Webster, A. (2002). Innovative health technologies and the social: Redefining health, medicine, and the body. Current Sociology, 50(3), 443-457. 
Wells, R.E., Yeh, G.Y., Kerr, C.E., Wolkin, J., Davis, R.B., Tan, Y., ... \& Press, D. (2013). Meditation's impact on default mode network and hippocampus in mild cognitive impairment: A pilot study. Neuroscience Letters, 556, 15-19.

Wesseling, L. (2002). Deskundige waarschuwingen tegen deskundigen: Een gemeenplaats uit de psychologiserende opvoedingsvoorlichting. In F. van Lunteren, B. Theunissen, and R. Vermij (Eds.), De opmars van deskundigen. Souffleurs van de samenleving (pp. 147-160). Amsterdam: Amsterdam University Press.

Wesseling, E. (2004). Judith Rich Harris: The Miss Marple of developmental psychology. Science in Context, 17(3), 293-314.

Wetherell, M., \& Potter, J. (1988). Discourse analysis and the identification of interpretive repertoires. In C. Antaki (Ed.), Analysing everyday explanation: A casebook of methods (pp. 168-183). Newbury Park, CA: Sage.

Wetherell, M. (1998). Positioning and interpretative repertoires: Conversation analysis and poststructuralism in dialogue. Discourse and Society, 9(3), 387-412.

West-Eberhard, M. J. (2003). Developmental plasticity and evolution. Oxford, U.K.: Oxford University Press.

Wexler, B. E. (2006). Brain and culture: Neurobiology, ideology, and social change. Cambridge, MA: MIT Press.

Williams, B. (1985). Ethics and the limits of philosophy. Cambridge, MA: Harvard University Press.

Williams, S. J., Higgs, P., \& Katz, S. (2012). Neuroculture, active ageing and the 'older brain': Problems, promises and prospects. Sociology of Health \& Illness, 34(1), 64-78.

World Health Organization. (2016). World report on ageing and health. Geneva: WHO.

Wren, D. A., \& Bedeian, A. G. (2008). The evolution of management thought. Hoboken, NJ: Wiley.

Wubs, J. (2004). Luisteren naar deskundigen. Opvoedingsadvies aan Nederlandse ouders 1945-1999. Assen: Koninklijke van Gorcum.

Wynne, B. (1992). Public understanding of science research: New horizons or hall of mirrors? Public Understanding of Science, 1(1), 37-43. 



\section{SAMENVATTING}

eurowetenschappelijke kennis over het brein wordt steeds vaker gebruikt
in verschillende maatschappelijke domeinen - van opvoeding en onderwijs tot management en zelfhulp. Deze verspreiding van kennis over het brein in de maatschappij is grotendeels te danken aan een specifiek en recent model van de hersenen. Onze hersenen worden sinds de jaren 1990 gezien als plastic, als veranderbaar en maakbaar. Het plastische brein maakt onze hersenen tot object voor allerlei interventies en het nodigt ons uit om er iets mee te doen. Menig neurowetenschapper en populariseerder van die wetenschap gebruikt de plasticiteit van het brein om te laten zien hoe we ons brein het beste kunnen verbeteren en hoe we ons leven het beste kunnen vormgeven om de potentie van het plastische brein te verzilveren. Met andere woorden: het plastische brein stelt neurowetenschappers en vertalers van die kennis in staat om ethiek te bedrijven.

Dit proefschrift onderzoekt hoe het plastische brein in verschillende maatschappelijke contexten precies gebruikt wordt om ethiek te bedrijven. Vragen over wat te doen, en hoe we het beste kunnen leven, staan centraal in de deugdethiek. In deze traditie van ethisch denken - die begon bij de oude Grieken en sinds de jaren 1950 weer is opgebloeid in het Westen - worden antwoorden gezocht op de vraag naar het goede leven: hoe kan ik floreren als individu, gegeven de mogelijkheden die ik heb als mens? Hoe word ik een goed persoon, een goede ouder, een goede werknemer, of een goede docent? Doel van dit proefschrift is om te laten zien hoe kennis over het plastische brein gebruikt wordt om dergelijke normatieve vragen te beantwoorden. Hoe worden de ethische implicaties van het plastische brein verwoord in maatschappelijke discussies over zelfverwerkelijking en het goede leven? En wat zijn de consequenties van het herformuleren van zulke ethische vragen in neurowetenschappelijke termen? Deze vragen staan centraal in dit proefschrift. 
In het inleidende hoofdstuk schets ik het probleem van plasticiteit als ethisch concept, en hoe voor- en tegenstanders in het academische debat rondom de maatschappelijke implicaties van de neurowetenschappen het plastische brein verschillend evalueren. Vervolgens ontwikkel ik een analytisch kader om normatieve argumentaties rondom het plastische brein te bestuderen.

Plasticiteit is een complex concept. Plasticiteit impliceert een wederzijdse beinvloeding tussen het individu, zijn brein en de omgeving. Daarnaast impliceert plasticiteit ook een vorm van robuustheid: in tegenstelling tot elasticiteit, verondersteld het plastische brein dat breinveranderingen een enigszins definitief karakter hebben. Door de interactiviteit en onbepaaldheid van het concept, verhult het plastische brein vragen die lang centraal stonden in sociologische en filosofische debatten over nature/nurture en agency/structure: wat kunnen we bewust veranderen aan onszelf, en wat moeten we accepteren als onveranderlijk? En wie kan er wat veranderen? Hoeveel agency en vrijheid geeft het plastische brein ons om onszelf vorm te geven? Gezien de conceptuele dubbelzinnigheid, is het wellicht niet vreemd dat meningen over de potentie en implicaties van een plastisch brein uiteenlopen. Optimisten zien in plasticiteit een vorm van emancipatie en bevrijding: het zou ons eindelijk voorbij de patstelling in het nature/nuture-debat brengen, doordat we nu kunnen praten over de invloed van sociale, culturele, technologische, gender of andere structurele factoren op onze biologie. De hoopvolle belofte van plasticiteit, die we niet alleen bij populaire interventies gebaseerd op het plastische brein vinden maar ook bij sommige academici uit de sociale- en geesteswetenschappen, is dat deze nieuwe kennis ons nieuwe mogelijkheden geeft om ons leven beter te leiden. Pessimisten daarentegen zien plasticiteit niet als een versterking, maar eerder als een bedreiging voor onze autonomie. Het plastische brein, en de belofte dat we daarmee op een nieuwe manier onszelf kunnen verbeteren, zou ons alleen maar meer verantwoordelijk maken voor problemen die eerder politiek of economisch van aard zijn.

Wat beide kampen gemeen hebben, zijn enkele assumpties ten aanzien van de maatschappelijke impact van het plastische brein. Beide posities in het debat veronderstellen dat neurowetenschappelijke kennis specifieke morele veranderingen met zich meebrengt (empowerment versus disciplinering), veranderingen die zich haast automatisch voltrekken. Beide posities slaan een denkstap over en negeren een aantal belangrijke vragen: hoe wordt kennis over het plastische brein überhaupt relevant en waardevol gemaakt? Welke problemen en idealen van het goede leven worden gearticuleerd en beloofd aan de hand van 
het plastische brein? Het is niet vanzelfsprekend dat neurowetenschappelijke kennis - kennis die hoogst technisch is en abstract - een rol speelt in het beantwoorden van vragen over hoe we moeten leven, laat staan dat ze deze ideeën en idealen veranderen. Dus de vraag is ook: hoe worden de ethische implicaties en de mogelijke morele veranderingen voorgesteld en al dan niet bewerkstelligd in de praktijk?

Om deze vragen te beantwoorden, richt ik me in dit proefschrift op een drietal maatschappelijke praktijken waar het plastische brein de laatste vijftien jaar zijn opgang maakte in antwoorden op vragen over wat te doen en hoe te leven. De drie praktijken zijn het puberbrein in de context van opvoeding, het volwassen brein in de context van werk en het ouder wordende brein in de context van zelfzorg. Ik vertrek daarbij vanuit een pragmatistische visie op ethiek en combineer theorieën en methodologieën uit het vakgebied Science E Technology Studies (STS), in het bijzonder sociology of valuations en sociology of expectations.

Ik focus daarbij op bepaalde ethische vragen en kwesties, namelijk kwesties die betrekking hebben op het goede leven - op wat het betekent om een goed ouder te zijn voor een kind met een puberend brein, hoe een goede werknemer te worden met een gestrest brein, en hoe goed oud te worden met een ouder wordend brein. Dat doe ik door te bestuderen hoe vertalers en populariseerders kennis over het plastische brein waardevol maken voor dergelijke vragen. Me baserend op recent werk in de zogenaamde sociology of valuations, noem ik die activiteiten waardenwerk. Waardenwerk is het proces van waarderen: het toeschrijven van waarde aan iets, maar ook het evalueren van iets als waardevol. Waarden, zoals geluk, gezondheid of creativiteit, zijn geen vaste entiteiten die stiekem ons gedrag sturen, maar zijn eerder de uitkomst van bepaalde (sociale en discursieve) processen van waardering, waar dergelijke waarden worden opgeroepen, gearticuleerd of betwist. De performativiteit en dubbelzinnigheid van het plastische brein nodigt de gebruikers van dat concept uit om waardenwerk te doen: om te articuleren hoe en voor welk wenselijk einddoel we ons brein zouden moeten veranderen.

Om dit waardenwerk te expliciteren kijk ik naar plekken waar wetenschappelijke kennis verspreid wordt: persberichten, nieuws in dagbladen, interviews met neurowetenschappers, magazines, populair wetenschappelijke boeken, etc. Op zulke plekken worden neurowetenschappers en andere neuro-vertalers uitgenodigd om kennis van het brein relevant en waardevol te maken. Wat we van het recente werk uit het onderzoeksveld sociology of expectations kunnen 
leren, is dat wetenschappers dat vaak doen door aan te sluiten bij bestaande problemen of dilemma's en door beloftes en verwachtingen te mobiliseren.

Bij het bestuderen van de manieren hoe kennis van het plastische brein waardevol wordt gemaakt, richt ik me specifiek op bepaalde waarderingen: waarderingen die te maken hebben met het goede leven. Ik ga op zoek naar herhalende patronen in de waardering van het plastische brein in praktijken van opvoeden, werken en ouder worden. Deze terugkerende waarderingen noem ik therapeutische repertoires. Met de pragmatistische theorieën van Swierstra (2011) en Nussbaum (1994) als uitgangspunt onderscheid ik vier elementen van zo'n therapeutisch repertoire van het goede leven: zelfkennis (bestaande uit diagnose en ideaal), praktische mogelijkheden tot zelf-stilering (actieprogramma's en vaardigheden die daarvoor nodig zijn), een visie op de wereld waarbinnen we kunnen floreren (van chaos tot orde) en tot slot aannames over wat we in staat zijn om te doen binnen zo'n wereld (van wil tot lot).

In de hoofdstukken die volgen, laat ik zie hoe de verspreiding van verschillende plastische breinen in verschillende praktijken resulteert in verschillende therapeutische repertoires van wat het betekent om respectievelijk een goed ouder, goed werknemer of goed ouder te worden met een plastisch brein. Elk van de drie casestudies benadrukt een bepaalde vorm van plasticiteit: agency toebedeeld aan de omgeving (puberbrein), het individu (volwassen brein) of het brein zelf (ouder wordende brein). Tegelijkertijd, zo zullen we zien, spelen in elke case alle facetten een rol (de omgeving, het individu en het brein). Plasticiteit wordt in elke case op verschillende manieren gewaardeerd en mondt uit in verschillende repertoires. Samen laten deze case studies zien hoe het plastische brein waardevol wordt gemaakt in verschillende fases van ons (goede) leven.

In hoofdstuk twee laat ik zien hoe het puberbrein wordt gebruikt door pedagogen en family coaches die ouders adviseren hoe ze hun puberkinderen het beste kunnen opvoeden. Ik plaats het puberbrein in de geschiedenis van het denken over adolescenten en de verwetenschappelijking van opvoedingsadvies. Ik expliciteer hoe de bekende Nederlandse neuropsycholoog Eveline Crone haar onderzoek naar het puberbrein relevant en waardevol maakt voor ouders. Ook traceer ik hoe pedagogen en andere opvoedingsdeskundigen haar populaire wetenschappelijke boeken over het puberbrein vervolgens waarderen. Het puberbrein wordt gezien als erg ontvankelijk voor omgevingsstimuli. Agency wordt hier in eerste instantie gegeven aan de omgeving die een vor- 
mende invloed zou hebben op het puberbrein. Er ontstaan twee verschillende therapeutische repertoires over opvoeden als externe prefrontale cortex. De ontvankelijkheid van het plastische puberbrein wordt verschillend geëvalueerd: de adolescent wordt gezien als "defect" individu met een kwetsbaar brein, maar ook als een vitaal en uniek individu, waarbij de openheid van het puberbrein gezien wordt als kans. Dit resulteert in verschillende en soms conflicterende opvoedadviezen, waarmee ouders aangespoord worden om wel of niet strikt te zijn, om lage of juist hoge verwachtingen te hebben van hun pubers, of om meer of minder controle op het puberbrein uit te oefenen. Samen reflecteren de twee repertoires de hedendaagse Nederlandse opvoedingsstijl, waarbij grenzen stellen en controle uitoefenen hand in hand gaan met begripvolle acceptatie en ruimte geven voor zelfontwikkeling. Het puberbrein in de context van ouderschap leidt daarmee niet tot substantiële morele veranderingen. Wel zien we een kleine verschuiving in de waardering van adolescenten: de idee dat een vorm van "vitaliteit" karakteristiek is voor de adolescentie - terug te voeren tot Aristotles en de psycholoog Hall - heeft nu een rijkere biologische onderbouwing gekregen en fungeert als tegenwicht voor een stereotypering van adolescenten als problematische wezens.

In hoofdstuk drie bestudeer ik de opmars van mindfulnesstrainingen op de werkvloer. Mindfulnessprogramma's en -boeken gebruiken vaak kennis over het (plastische) brein om de voordelen van deze van oorsprong boeddhistische vorm van meditatie aan te prijzen. Ik laat zien hoe neurowetenschappers hun onderzoek naar meditatie en het brein waardevol maken, en hoe mindfulnesstrainingen en -boeken voor werknemers en managers deze kennis vervolgens oppakken en waarderen. Het volwassen, stressvolle brein wordt gezien als $o b$ ject van verandering; agency wordt hier toegeschreven aan het individu dat in staat wordt gesteld om zelf het brein te veranderen. Er ontstaan wederom twee verschillende therapeutische repertoires over goed werken met mindful hersenen. De twee repertoires in deze case evalueren de doelen van deze breinverandering verschillend. In het eerste repertoire worden zelfgerichte breinveranderingen gezien als het optimaliseren van individuele productiviteit en efficiëntie. In het tweede repertoire worden naast een betere balans tussen werk en privé, ook de sociale effecten van breintraining benadrukt, zoals empathie, vriendelijkheid en een socialere bedrijfscultuur. De twee repertoires samen verklaren waarom mindfulness op de werkvloer niet resulteert in substantieel veranderende ideeën en praktijken van wat het bekent om een goed werknemer 
te zijn. De ideale werknemer in mindfulness programma's combineert emotionele zelfcontrole met sociale zelfverwerkelijking. Daarmee belooft mindfulness werknemers en managers dat ze geen keuze hoeven te maken in de soms tegenstrijdige eisen die werken in onze hedendaagse kenniseconomie veronderstelt.

In hoofdstuk vier onderzoek ik hoe het ouder wordende brein gebruikt wordt om te articuleren wat goed ouder worden zou moeten zijn. Het ouder wordende brein wordt gezien als onderhevig aan interne veranderingsprocessen; agency wordt hier toegeschreven aan het veranderende brein zelf. Ik volg de controverse rondom brain games: populaire computerspelletjes waarbij senioren worden aangespoord om hun oudere hersenen te blijven trainen. Deze brain games vormen een soort ethisch ijkpunt in het publieke debat over goed ouder worden met een verouderend brein. Nadat ik expliciteer hoe senioren worden aangesproken door de makers van zulke breinspelletjes, laat ik zien hoe gerontologen, auteurs van zelfhulpboeken en anderen zich afzetten tegen de opgeroepen beloftes en verwachtingen. In de twee therapeutische repertoires van goed ouder worden met een plastisch brein worden zowel de beloftes van breinspelletjes alsook het onderliggende ideaal van goed ouder worden bekritiseerd. Actoren gebruiken het ouder wordende brein om hedendaagse en traditionele visies van goed ouder worden te combineren, waardoor het idee van ouderdom niet substantieel verandert. De repertoires samen reflecteren hoe we door de geschiedenis heen over ouderdom denken: als een periode van cognitieve achteruitgang, maar ook als een periode waarin de vruchten van een levenslange ervaring geplukt kunnen worden (wijsheid). Net als in de casus over het puberbrein ontstaat ook hier een rijker vocabulaire om ouderdom als een levensfase te zien met unieke, en wenselijke eigenschappen. Dat komt echter wel met een prijs: het plastische, ouder wordende brein blijft een hoopvol verhaal, en wordt niet gebruikt om senioren voor te bereiden op ziekte, lijden, en het onvermijdelijke.

In het concluderende hoofdstuk reflecteer ik op mijn bevindingen en plaats ik deze in een bredere context. De belangrijkste bevinding van dit proefschrift is dat het gebruik van het plastische brein niet resulteert in substantiële veranderingen in wat het betekent om een goede opvoeder, goede werknemer, of goede ouder te zijn. Keer op keer zien we dat het plastische brein waardevol wordt gemaakt in verschillende (en soms conflicterende) contextspecifieke repertoires van respectievelijk goed ouderschap, goed werknemerschap en goed ouder worden. Het plastische brein kan dan ook het beste beschouwd worden als een 
projectiescherm dat hedendaagse uitdagingen, ontwikkelingen en (morele) veranderingen in culturele tradities en praktijken van opvoeden, werken en ouder worden reflecteert.

De ethische ambiguiteit van het plastische brein stelt gebruikers van breinkennis in staat om het brein op verschillende manieren waardevol en relevant te maken, afhankelijk van de morele positie die men wil articuleren. Dit betekent echter niet dat er geen enkele normatieve effecten in de bestudeerde praktijken waar te nemen zijn. In plaats van nieuwe zelfkennis, nieuwe actieprogramma's of nieuwe idealen, zien we wel enige veranderingen in de hegemonie van posities in de betreffende praktijken. Het concept van het plastische brein stelt haar gebruikers in staat om dominante posities aan te vechten en alternatieven voor te stellen. Morele veranderingen nemen de vorm aan van verschuivingen in de relaties tussen elementen van het goede leven, en in het relatieve gewicht van morele posities in een publiek debat.

In plaats van het eenduidig beantwoorden van ethische vragen en het oplossen van uitdagingen in deze praktijken - de beloftes van voorstanders van plasticiteit - zien we eerder een verplaatsing van deze ethische vragen en uitdagingen naar het plastische breindiscours zelf. Deze verplaatsing van ethiek naar het brein is een gevolg van het waardenwerk dat actoren verrichten met het concept van het plastische brein. Hoe actoren problemen en oplossingen articuleren en propageren aan de hand van het plastische brein, hangt in grote mate af van de manier waarop determinisme en agency toegeschreven worden aan het individuele subject, zijn brein of de omgeving. In deze toebedeling van agency zien we enkele terugkerende patronen.

De ethische ambiguiteit van plasticiteit speelt zich af op twee niveaus: binnen en tussen de therapeutische repertoires. Binnen therapeutische repertoires zien we een dans van agency. Door verschillende strategieën van waardenwerk wordt agency eerst toebedeeld aan de omgeving of het brein (diagnose), vervolgens aan het individuele subject (actieprogramma), om daarna weer terug te vloeien naar het brein (ideaal). Ook tussen de therapeutische repertoires kunnen we een herhaling van zetten ontwaren. De bifurcatie van twee repertoires is niet toevallig. Hoewel elk repertoire het individuele subject aanspreekt om zijn brein te oefenen, is er in elke case ook een repertoire ontstaan dat een appel doet op de omgeving van het individu. Op de aloude vraag 'Wie moet wat veranderen?' geven de twee repertoires in elke casus dus een iets ander antwoord: het individuele subject alleen, of het individuele subject samen met de omgeving. In tegenstelling tot de belofte dat plasticiteit ons voorbij nature/nurture en agen- 
cy/structure debatten zou brengen, blijkt het concept in de praktijk niet sterk genoeg om de actoren door deze ingesleten, dualistische denkpatronen te laten breken. Het is via het plastische brein dat individu en omgeving worden aangesproken als zowel object als agent van verandering. Het is via het plastische brein dat actoren hopen op zowel stabiliteit als verandering.

De uitdagingen en idealen die geformuleerd worden aan de hand van het plastische brein veronderstellen een maatschappij die zich kenmerkt door snelle en onvoorspelbare veranderingen - veranderingen die zich goeddeels afspelen buiten de invloedsfeer van het individuele subject. Het plastische brein weerspiegelt de behoefte naar het vinden van harmonie met een veranderende wereld. Het plastische brein gaat in elke casus over het cultiveren van verandering: van zelfregulering, flexibiliteit en veerkracht tot normaliteit, creativiteit en socialiteit. Deze idealen van plasticiteit impliceren dat het omgaan met veranderingen een nieuwe deugd is geworden in onze hedendaagse maatschappij: goed voor het individuele subject, maar ook voor de samenleving.

Het plastische brein spreekt het individuele subject aan op zijn ethische taak om zelf zorg te dragen voor het eigen leven - en wel door het brein te oefenen. In tegenstelling tot klassieke deugdethische theorieën komt het plastische brein in de praktijk niet met een substantiële kritiek op onze maatschappij. Het plastische brein is begrensd door hoop: verhalen over lot, tragedie of onrechtvaardigheid worden niet verteld. Om die reden wijzen critici op het gevaar dat interventies gebaseerd op het plastische brein al snel uitmonden in een perverse maatschappelijke verplichting. Deze zorg is terecht. Toch kan er een kleine kanttekening gemaakt worden. Omdat waarderingen van het plastische brein in de praktijk niet louter individualistisch zijn - ook de omgeving wordt aangesproken en dient te veranderen (juist zodat het plastische brein kan floreren) kunnen we niet langer emancipatie of disciplinering theoretisch veronderstellen. In plaats daarvan zouden we de vraag moeten stellen: wanneer en onder welke condities is het aanspreken van een individu op de ethische taak om zorg te dragen voor haar eigen leven, te veeleisend?

Naast deze kanttekening zijn er nog twee implicaties voor het bestuderen van de maatschappelijke effecten van neurowetenschappelijke kennis. De eerste is dat actoren in de praktijk enige vrijheid hebben in de manier waarop ze breinfeiten waardevol kunnen maken. De waarde van het plastische brein komt niet automatisch uit het lab gerold en is ook geen simpel uitvloeisel van onze hedendaagse neoliberale ideologie. Het is een resultaat van praktische en contextuele processen van waardering. Het negeren van deze activiteiten van 
waardenwerk kan resulteren in het over het hoofd zien van de veelheid en verwevenheid van breinfeiten en waarden in de praktijk. Dat betekent (ten tweede) dat we in onze theorieën over de maatschappelijke impact van (neuro)wetenschappelijke kennis niet a priori kunnen veronderstellen of er al dan niet morele veranderingen optreden. Dat is een onderwerp voor empirisch onderzoek.

In onze hedendaagse, technologische samenleving zijn vragen over hoe (goed) te leven steeds vaker onderwerp van publiek debat. Met dit boek heb ik geprobeerd te laten zien hoe we dergelijke vragen te lijf gaan met recente neurowetenschappelijke kennis. Dat wetenschappelijke kennis niet in een vacuüm ontstaat of waardevol gemaakt wordt, maar onlosmakelijk verbonden is met waarden, kan niet genoeg herhaald worden in deze tijden van fact-checking en post-truth. Het expliciteren van de manieren waarop wetenschappers en vertalers van die wetenschap engageren met waarden kan helpen om wetenschappers gevoeliger te maken voor de mogelijke maatschappelijke effecten van hun eigen onderzoek. Daarnaast laat het zien waar wij, geestes- en sociaal wetenschappers, onze kritiek op moeten richten: op het verborgen werk dat nodig is om wetenschappelijke kennis waardevol te laten zijn, maar ook op onze bereidheid om bepaalde morele verhalen te accepteren ten koste van andere. 



\section{THE VALUE OF VALUE WORK: \\ A NOTE ON VALORISATION}

$\mathrm{T}$ this dissertation showed what efforts it takes for neuroscientists, science journalists, and other science translators to make brain claims relevant and meaningful for non-neuroscience audiences. Scientific claims do not speak for themselves: They have to be made relevant and valuable, through strategies of value work. It's through these activities that scientific claims are made to (dis)align with context-specific challenges and ideals. The main merit of this dissertation is that it shows how such valuations of science actually work in different societal practices. In the case of the plastic brain, such valuations are closely connected to challenges and ideals of the good life.

To acknowledge that articulating the societal value of scholarly work takes effort, is not something new. Spinoza, for example, argued not only that scholars should use the latest sciences in the quest for achieving the highest good. He also stressed the importance for scholars to take the effort "to speak in a manner intelligible to the multitude" (Spinoza, 1662/1997, 17:2). Today, universities, funding agencies, and science policymakers rather use the term valorisation to urge scholars to articulate the relevance and value of their own scientific research to non-scientific audiences.

As the only university in the Netherlands, Maastricht University requests that PhDs include a valorisation addendum in the dissertation. In this addendum, I reflect on the societal relevance of my dissertation, and on my own value work. For whom could I make my research valuable? And how did I make my research relevant and valuable for audiences other than philosophers or scholars in Science and Technology Studies (STS)? After a brief overview of my past value work activities, I reflect on the potential value of my dissertation for dif- 
ferent audiences. I end with a short note on the value of value work for the contemporary public and scholarly debate on valorisation.

\section{Engaging the Multitude: “But I'm just interested in the brain!"}

During my PhD trajectory, I gave several public presentations and lectures. Especially in the first years of study, these presentations often took the form of a critical, philosophical assessment of the promises of neuro-advocates. For example, at the science festival Discovery at MU artspace (Eindhoven, 2013) and again at a PechaKucha Night at the Temporary Art Centre (Eindhoven, 2013), my presentations were titled "How to Resist Brain Porn? 3 Amazing Secrets to Boost Your Understanding of the Neurosciences! + 1 Bonus Secret!" For the popular Dutch blog Vice Motherboard, I wrote an essay on neurohype, explaining fMRI, statistics, and correlation vs. causation issues. Especially at the height of the public neuro-turn in society (roughly between 2010 and 2015 in the Netherlands), to spur some (neuro)scientific and philosophical literacy in the ironic form of self-help advice seemed a worthwhile endeavour as a philosopher/STS scholar studying neuroscience.

I thus was problematizing neurohype - showing the methodological and conceptual complexity behind popular brain claims. I aimed to shield the uninformed from the "seductive allure" of the neurosciences by showing how to separate bad neuro-reasoning from good neuro-reasoning. But I became wary of this role of the philosopher as a superior referee between good and bad science. I realised that this tactic was based on a problematic assumption: The popularity of brain claims on all issues of life was not only a matter of overshooting promises - it was just as much about the expectations science journalists and audiences had about the scope of the contemporary neurosciences. I came to think of neurohype not as an issue about truthfulness, but rather as a matter of societal utility and desirability.

I changed tactics. Instead of focusing on what was philosophically problematic in neuroscience research and its translations, I started to emphasize what it took for neuro-advocates to successfully disseminate their technical knowledge to a wide range of audiences. And instead of problematizing the lack of scientific literacy of the general audience, I started scrutinizing the need for this knowledge and the expectations we have regarding the contemporary neuro- 
sciences. Where does our willingness to embrace certain neuroscientific claims come from?

When my research advanced, I could share my focus and findings with different audiences. I presented my early findings on value work to students of psychology and neuroscience at the Science College Maastricht, University of Groningen, and to neuroscientists and other scholars dealing with the neurosciences at a number of interdisciplinary conferences (hosted by, for example, the University of Pennsylvania, US; Johannes Gutenberg-University in Mainz, GE; University of Amsterdam, NL; and University of Edinburg, UK). The topic of the hidden moral labour of science translation found a willing and eager audience among neuroscientists and others whose interdisciplinary work engages with the neurosciences. To reach a more general audience, I wrote a column on value work in self-help literature in the popular philosophy magazine Wijsgerig Perspectief (2018).

\section{To parents, employees and seniors: using science to articulate the good}

The general thrust of my argument is that scientific knowledge and values are closely entangled, especially when scientific claims spread beyond the lab, and are used for questions of practical judgement. While a clear distinction between facts and values is regarded untenable by many philosophers of science and scholars in STS, it is still part of the conceptual toolkit of scientists, science journalists, and the general audience. What I aimed to show in this dissertation, is that our willingness to accept (neuro)science knowledge is not only determined by its truth-value, but rather by its usability in our practices.

A more nuanced understanding of how scientific knowledge plays a role in articulating the good, can benefit the scientific and ethical literacy of the audiences addressed in my case studies. Readers of expository works and practitioners of self-help programs on the plastic brain, should be aware that (neuro)scientists and translators of that science mobilize (everyday, societal) problems and ideals in order to put neuroscience knowledge forward as a solution. In doing so, science translators have to appeal to the familiar and, at the same time, propose a change or something new. As we have seen, all too often the problems addressed and ideals invoked, are remarkably familiar. The proposed change or newness is often to be found in a different (in this case: neuroscien- 
tific) perspective on a problem and a corresponding solution (i.e. changing the brain).

This value work does not make scientific claims less true, nor does it mean that parents, employees or seniors should not use such popular science work while engaged in the ethical task of improving one's life. It does mean that readers of such popular science works should not expect a conclusive answer to their problems. The chapter on the teenage brain as parenting advice, can help parents and pedagogues understand that they should not expect that new knowledge of adolescent brain development will give them a final - or even a new - answer to the pedagogical challenges of raising a teenager. Instead, this case study urges parents and pedagogues to ask themselves what they expect from knowledge of adolescent brain development, and for what reason and to what end this knowledge could play a role in their lives. They should question how knowledge of the teenage brain can help them to become the kind of parents they want to be.

Similarly, the chapter on the mindful brain at the work floor makes clear to employees and managers that the mindful brain is not a neutral instrument to counter stress. This does not mean that employees and managers who adopt mindfulness to practice their plastic brain in the workplace, should dismiss such mindfulness programs, merely because there are different valuations of the mindful brain in practice. What this chapter makes clear, is that they should keep asking themselves the reason why they practice mindfulness-as-braintraining, and whether the solutions and problems mobilized in mindfulness programs correspond adequately with their experience of work-related stress.

Seniors too, should keep a close eye on their own expectations of interventions that promise to change their ageing brains. Seniors should be wary of the promise of a quick fix to age-related concerns. Not only because such interventions lack in truthfulness, but to acknowledge the reason why such interventions could be desirable for them. Doing physical exercises or engaging socially can be worthwhile activities on their own, without aspiring to retain a youthful performance or to prevent cognitive decline. Seniors should acknowledge that new knowledge of ageing probably won't change the ambiguity of getting older: facing inevitable cognitive decline, while reaping the fruits of an experienced life.

My case studies can thus help audiences to better understand that (neuro)scientific knowledge in practice can be made valuable in multiple ways. Knowledge of the plastic brain can play a helpful role in articulating challenges 
and ideals, provided that audiences acknowledge that they cannot expect conclusive answers.

\section{To (neuro)scientists and science journalists: cultivate your moral labour}

Science is considered by many the realm of rationality and truth, whereas ethics is seen as the realm of emotion and confusion. As public debates on climate change or fake-news indicate, to problematize this stereotypical view easily results in either a distrust of scientists, or a staunch, positivist defence of science. The notion of value work can potentially give science translators a language (or tool) to acknowledge the co-shaping of science and ethics, without resorting to these stereotypical positions.

The co-shaping of science and ethics in practices of self-fashioning does not necessarily result in a naturalistic ethics, nor in an "anything goes" relativism. On the contrary: It shows the tremendous amount of effort it takes to make a scientific claim robust. As Wiebe Bijker argued in his valedictory lecture, scientific claims are special (compared to mere opinion) precisely because they are the outcome of all kinds of social mechanisms: peer-review, conferences, and funding committees such as the Dutch NWO (Maastricht University, 2017). Next to these institutionalized mechanisms, I have shown that there are also moral processes (value work) in play when valorising such claims to specific audiences. Science journalists play an important role in this societal process of knowledge formation.

Science journalists, and scholars in the humanities or social sciences who take up neuroscience knowledge, should be aware of the values and ideals they (implicitly) invoke to make such knowledge relevant. Especially science journalists should acknowledge and explicate the moral labour they employ in making scientific knowledge relevant for different audiences. Science journalists cannot hide behind the veil of truth, but instead should train their ethical sensitivity in order to critically assess how scientists make their work valuable, and how in turn their own translations of science reiterate or (dis)align with the dominant status-quo. Acknowledging that engaging with values is part of the work of science translation, can potentially contribute to a more open and thoughtful translation of science to societal practices. 
This goes, to a lesser extent, also for (neuro)scientists themselves. One could think that the notion of value work - which implies acknowledging that science is more than simply discovering and relaying facts of nature - would upset neuroscientists, who presumably would "only be interested in the brain." But nothing could be further from the truth. At conferences, a number of scholars admitted to me feeling awkward while writing their funding proposals, doing interviews, or reading the press releases their University press offices had drafted about their research. Most neuroscientists (such as Eveline Crone in this dissertation) are well aware of the limitations of their field and the high expectations of science journalists and the general public. At the same time, neuroscientists face pressures to quickly publish, to promise results in order to receive grants, and to share their research with the general audience. The notion of value work can give voice to these concerns and can contribute to an increased understanding among (neuro)scientists that values and cultural traditions necessarily must be invoked when they make their knowledge claims relevant for different audiences. The notion of value work can make the ethical activities visible that contemporary neuro(scientists) are expected to perform under the concealing label of valorisation.

\section{Valorisation as Academic Virtue?}

The often ill-defined term valorisation hides expectations and assumptions about the place, role, and ideals of the contemporary scholar in our society. Many academics have voiced their concerns about and critiques of the valorisation trend (cf. Older, 2014). ${ }^{61}$ During my PhD-trajectory I contributed to this debate as a member of the workgroup of the Platform Hervorming Nederlandse Universiteiten (HNU). We organised critical yet playful events to raise awareness and to stir up the public debate on a variety of issues in our contemporary academic climate (such as temporary contracts, work pressure, corporate marketing at universities, and the economisation of academic discourse). ${ }^{62}$ I also contributed to the drafting of our own faculty's policy on valorisation as a member of the Valorisation Committee.

\footnotetext{
${ }^{61}$ For an excellent analysis of Dutch views on valorisation, see the master thesis of Dani Older: Beyond bullshitting: Valorisation and the humanities in the Netherlands (Older, 2014)

${ }^{62}$ For a more substantial critique, read the Manifest of Platform HNU. (Halffman \& Radder, 2015), available at www.platform-hnu.nl.
} 
My aim in these discussions was to point out that valorisation should not be reduced to concrete and quantifiable forms of economic knowledge utilization. Nor should valorisation be seen as simply a matter of science communication belonging to the ever-increasing marketing departments at universities. This does not mean that we should dismiss valorisation altogether. We have to broaden our scope of what valorisation means in practice. More importantly, we have to become aware of the hidden moral labour it takes in practice to successfully valorise one's work. It starts by acknowledging that valorisation requires an ethical sensitivity of the actors involved.

This is not something radical or new. If we glance over the history of science as vocation, speaking intelligible to the multitude - as Spinoza famously put it has always been a task of the scholar as public intellectual. ${ }^{63}$ With the notion of value work, I hope to add a more practical, ethical, and substantial perspective to the discourse on valorisation in academia and beyond. The heightened awareness of and discussion about what comes to count as true in public debates - e.g., debates about post-truth, fake news or "alternative facts" - gives some topical urgency to do this. My message would be simple: In our complex society, the desire for truth cannot be easily separated from the desire - of both scientists and audiences - to do and be good.

${ }^{63}$ For an historical overview, see for example Shapin's The Scientific Life: A moral history of a late modern vocation (2009). 



\section{ABOUT THE AUTHOR}

$\mathrm{T}$ ies van de Werff (Gemert, 1981) received an MSc in Health Sciences (19992005), an MA with honours in Arts and Culture (2005-2007), and an MPhil in Cultures of Art, Science, and Technology (2007-2008) at Maastricht University (the Netherlands). After his studies, Ties worked as a freelance writer, editor, and curator, specialized in the intersections between art, science, and technology. Together with his partner he founded the social design collective Tante Netty in the Eindhoven district Woensel-West. Ties taught at the Faculty of Arts and Social Sciences (FASoS) at Maastricht University from 2009 to 2011 before starting his PhD trajectory at the Department of Philosophy there. As part of his PhD work, Ties was enrolled in the Netherlands Graduate School of Science, Technology, and Modern Culture (WTMC). He is also a member of the Centre for Ethics \& Politics of Emerging Technologies (EPET) and the Society for Social Studies of Science (4S).

In 2016, Ties was appointed as a researcher at the research centre for Arts, Autonomy, and the Public Sphere at the Faculty of the Arts in Maastricht (Zuyd University of Applied Sciences), where he currently studies the performance of values in engagement practices in the arts. In 2018, Ties has also taken up a post-doctoral position at FASoS, exploring innovative participatory practices in contemporary symphonic music orchestras. Moving between the loci of academia and the art world, Ties' research currently focusses on exploring contemporary forms of encultured ethics, cultivating an ethical sensitivity amongst artists, musicians, and scientists alike. Ties lives in Eindhoven with his partner and daughter. 
Despite its complex scientific technicalities, in the past decade knowledge of the brain has spread from the laboratory into a variety of societal domains. This book examines how translators and popularisers of neuroscience knowledge engage with values in order to make their brain claims relevant and valuable.

Key to our emerging 'neuroculture' is the concept of brain plasticity: our brains are now imagined as changeable, and open to interventions. The idea of a plastic brain allows neuroscience translators and popularisers to promise and propose new ways of changing our brains for the better - enhancing the good and preventing the bad. Combining theories and methods from Science and Technology Studies (STS), pragmatist ethics, and the sociology of valuation, this book empirically traces valuations and normativities of the plastic brain in three different societal practices of a good life: parenting, working, and ageing. It shows for what sorts of problems, prescriptions, and ideals the plastic brain is mobilized in these cases of a braininspired good life, and whether this results in changes in what it means to be a good parent, a good employee, or to age well in our contemporary society. 